\title{
Corrosion Behaviour of High-Carbon High-Molybdenum Stellite Alloys in Amine Environment
}

by

\author{
Kafeel Kamal
}

A thesis submitted to the Faculty of Graduate and Postdoctoral Affairs in partial fulfillment of the requirements for the degree of

Master of Applied Science

in

Materials Engineering

Carleton University

Ottawa, Ontario

(C) 2018, Kafeel Kamal 


\section{Abstract}

Stellite alloys are cobalt-based alloys which display exceptional mechanical properties, wear and corrosion resistance owing to its unique chemical compositions. Stellite 6 is the most popular Stellite alloy employed in various applications, one of which is the hardfacing of valve trim components within control valves in boiler feedwater treatment systems where hydrazine and/or amine derivatives are used. Erosion-corrosion caused failure of Stellite 6 hardfacing has been reported from industry in such applications. To find a better replacement of Stellite 6 for improved corrosion resistance in amine environment, 700 series Stellite alloys, including Stellite 706, Stellite 712 and Stellite 720, are proposed, which are high-carbon high-molybdenum Stellite alloys. The corrosion performance of these alloys in morpholine solution with $\mathrm{pH} 9.5$, which simulates the corrosive environment of boiler feedwater service, is evaluated using a series of electrochemical tests such as electrochemical impedance spectroscopy (EIS), potentiodynamic polarization and potentiostatic polarization. Both regular polarization tests (potential up to $1.2 \mathrm{~V}$ ) and failure polarization tests (potential up to $12 \mathrm{~V}$ ) are conducted on the alloys. The former identifies general and localized corrosion behaviour, while the latter intends to fail the alloy surface to analyze their limitations. Two different surface conditions (intact and damaged) and two different temperature variables $\left(25^{\circ} \mathrm{C}\right.$ and $\left.50^{\circ} \mathrm{C}\right)$ are also studied to simulate erosion-corrosion behaviour. Stellite 6 alloy is also tested under the same condition for comparison. It is shown that these 700 series Stellite alloys are comparably better than Stellite 6 with respect to oxide film stability, all exhibiting excellent corrosion resistance in morpholine solution with $\mathrm{pH}$ 9.5. The protective chromium oxide film on their surfaces is retained well for both regular and failure tests. These alloys behave similarly, however, Stellite 720 containing the highest carbon and molybdenum contents performed better at room temperature and Stellite 706 with the least carbon and molybdenum 
contents performed better at the elevated temperature. Although wear changes the surface condition, the corrosion resistance of the worn alloys is similar to that of intact alloys in the tested solution. The outcomes of this research suggest that 700 series Stellite alloys are good candidates for replacing Stellite 6, where Stellite 720 displayed the best performance under the conditions tested. 


\section{Acknowledgements}

I would first like to extend my deepest gratitude to my thesis supervisor Professor Rong Liu for giving me the opportunity to do MASc. I sincerely appreciate her confidence in my ability to carry out the research work and thank her for her guidance and patience during the writing stage of my thesis.

My sincerest thanks to Professor Glen McRae for mentoring me throughout my entire research period. His continuous guidance and support in this research made it possible to complete my work and gave me great insight on corrosion science.

I would also like to thank Steve Truttmann, David Raude and Alex Proctor for their assistance and training on specific equipment usage and material preparation. A special thanks to Kyle Fournier for his guidance and knowledge during my research work.

I would like to thank my friends and colleagues, Amin Ghaziaskar, Leigh Corrigall, Peter Walker, Lee Seung Hwan, Shah Khosraviani, Xiaozhou Zhang and Geoff Seatter for keeping me level headed and making these past two years a memorable one.

And finally, I would like to thank my family for their encouragement and moral support. 


\section{Table of Contents}

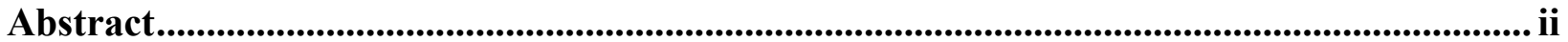

Acknowledgements ............................................................................................................................. iv

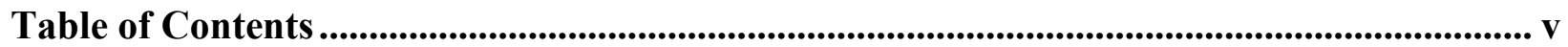

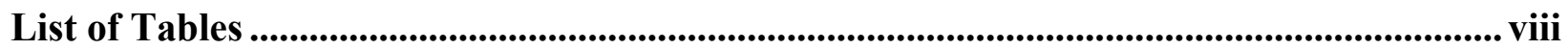

List of Figures........................................................................................................................................... ix

List of Acronyms ............................................................................................................................................ xvi

1 Chapter: Introduction ................................................................................................................ 1

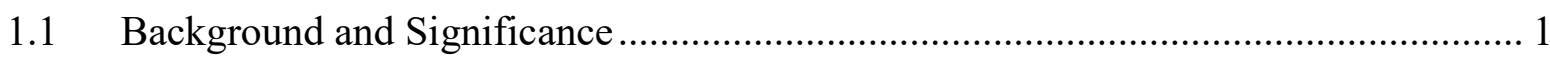

1.1.1 Synopsis of Stellite alloys ........................................................................ 1

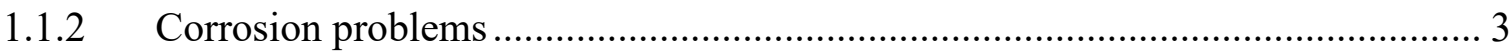

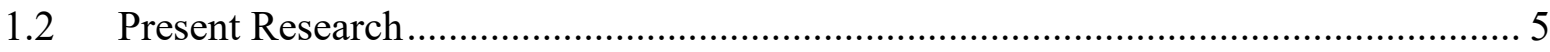

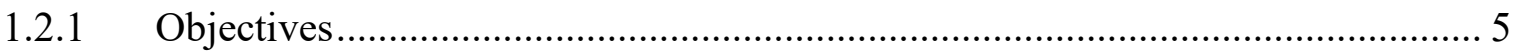

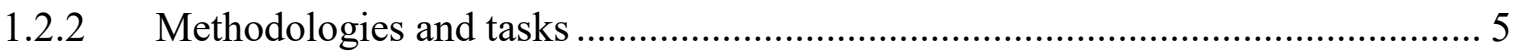

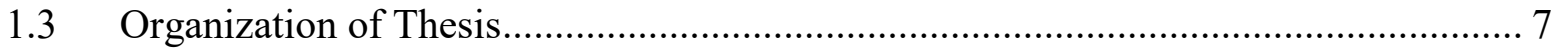

2 Chapter: Literature Review ................................................................................................... 8

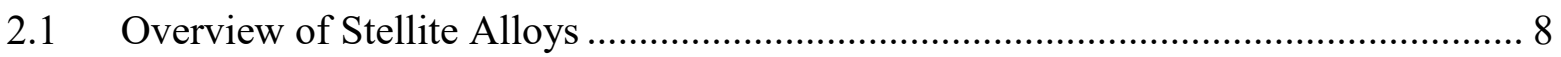

2.1.1 Chemical composition and microstructure.................................................... 8

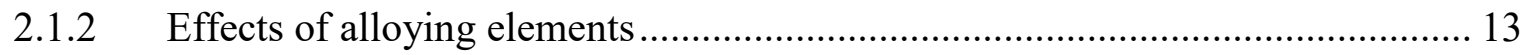

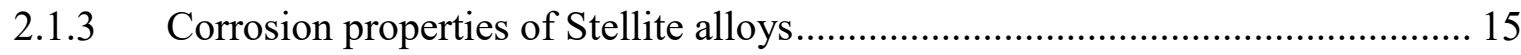

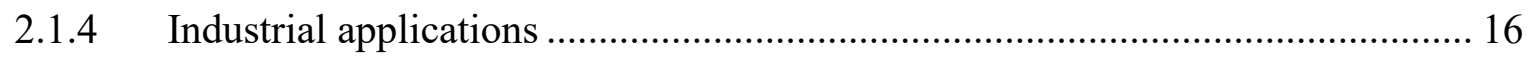

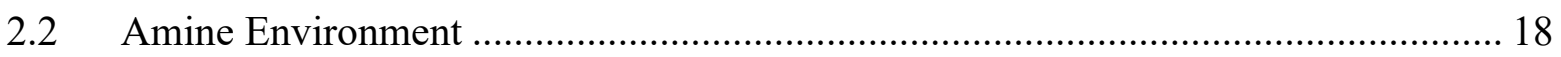




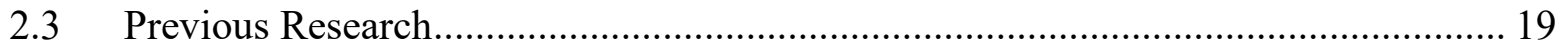

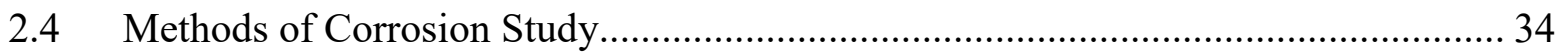

2.4.1 Electrochemical impedance spectroscopy (EIS) …………………………..... 34

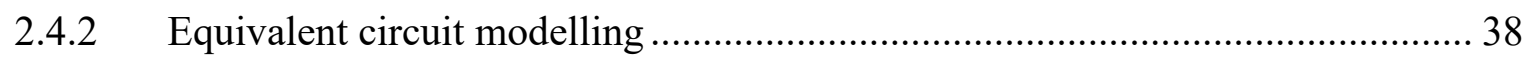

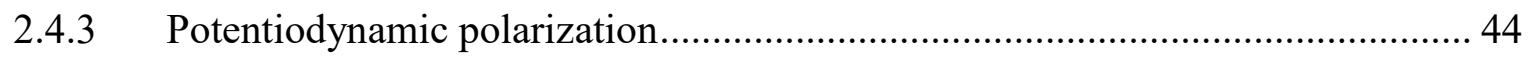

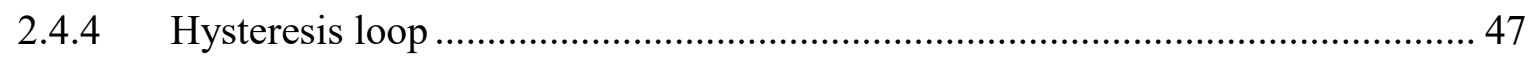

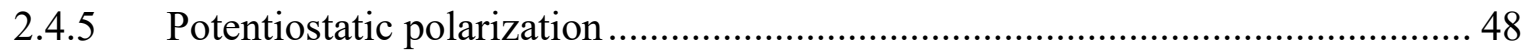

3 Chapter: Experimental Details ............................................................................................ 49

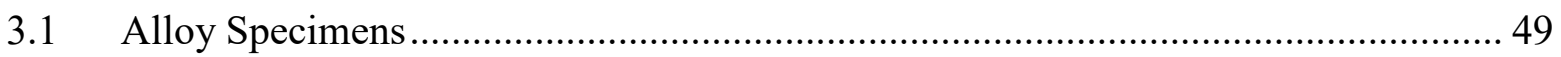

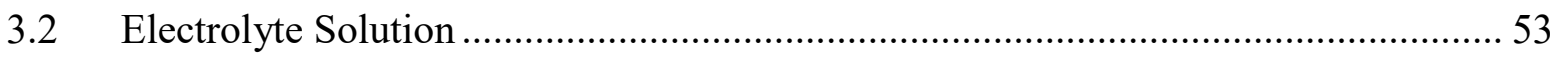

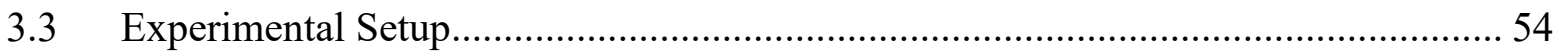

$3.4 \quad$ Experimental Procedure ……………………….................................................. 56

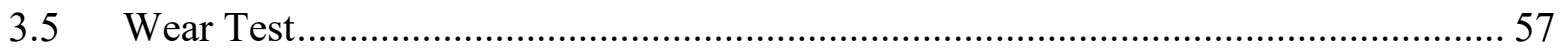

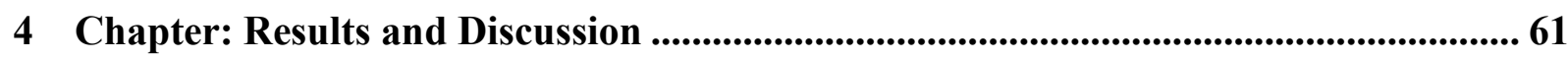

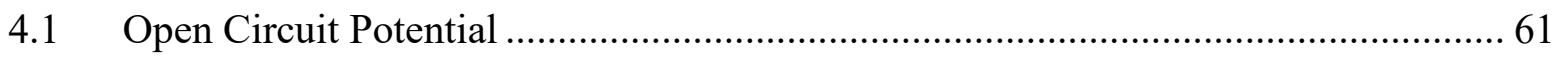

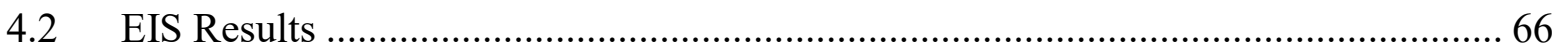

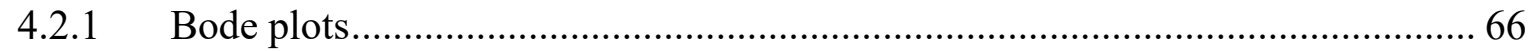

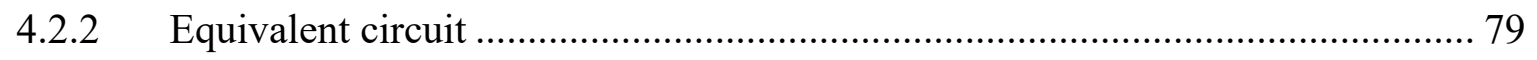

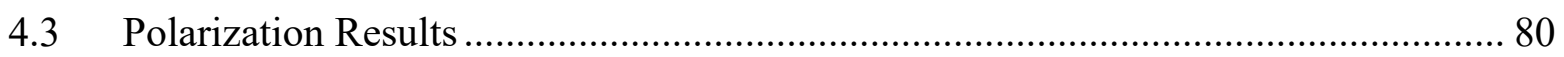

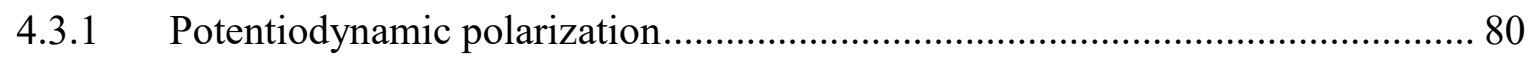




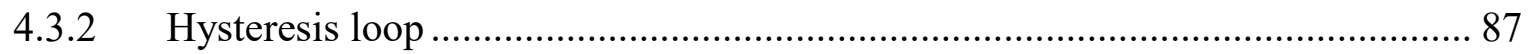

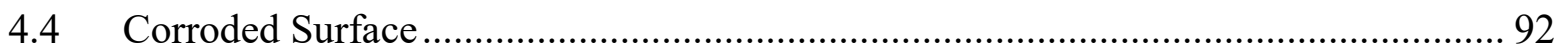

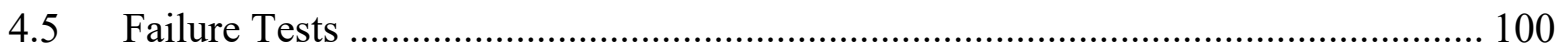

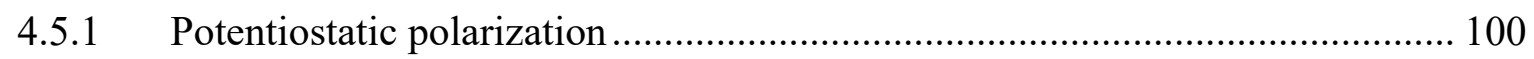

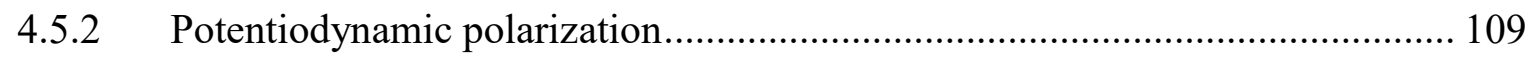

4.5.3 Corrosion performance in 3.5 wt. $\% ~ \mathrm{NaCl}$................................................ 112

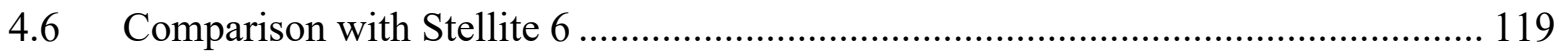

4.6.1 Chemical composition and microstructure..................................................... 119

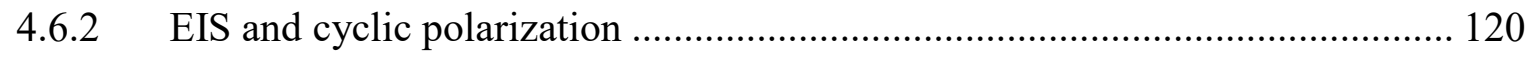

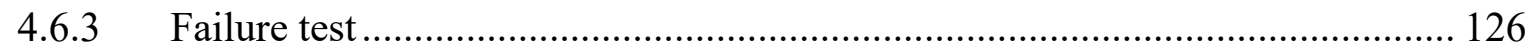

4.6.4 Overall comparison ............................................................................... 129

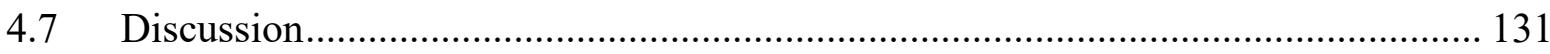

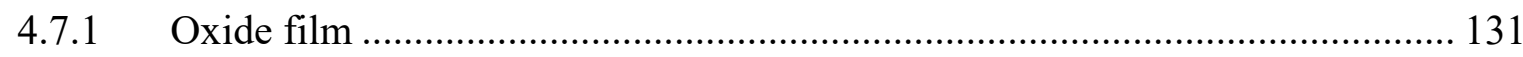

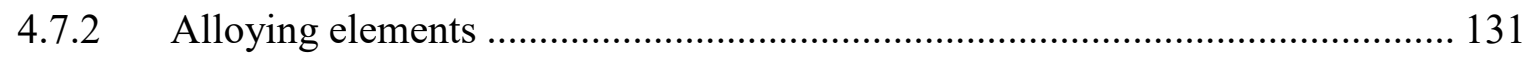

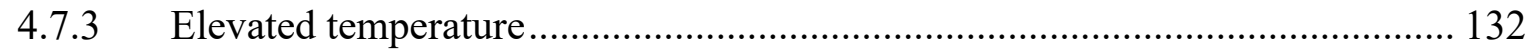

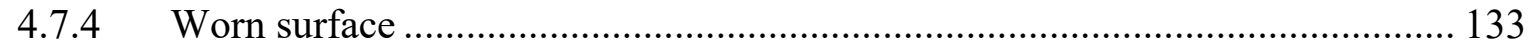

4.7.5 Pourbaix diagram ……………………………..................................... 133

5 Chapter: Conclusions and Future Work ....................................................................... 136

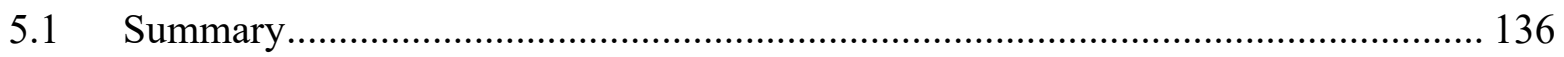

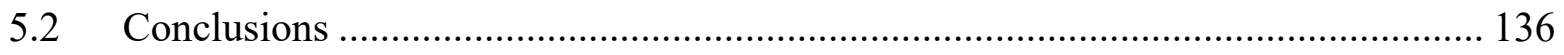


5.3 Research Implications.

5.4 Future Work 140

5.4.1 Process fluid flow rate, temperature and pressure. 140

5.4.2 Chemistry of the oxide film 140

5.4.3 Comparison with different amines . 141

5.4.4 Stellite 6 and variable $\mathrm{W}$ based compositions. 141

References 142

\section{List of Tables}

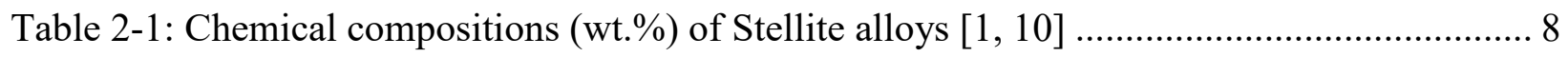

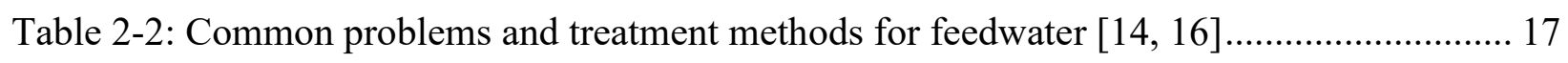

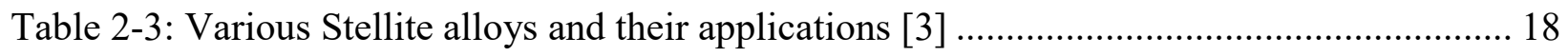

Table 2-4: EIS data for as-cast and HIPed Stellite 6 in 3.5 wt.\% $\mathrm{NaCl}$ solution [18] ................ 23

Table 2-5: Polarization parameters of Stellite 6 and 17-4PH stainless steel in basic environments 24

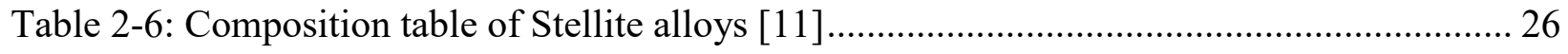

Table 2-7: Polarization parameters of Stellite alloys in 3.5 wt.\% $\mathrm{NaCl}$ solution [11] ............... 26

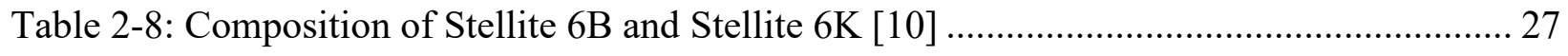

Table 2-9: Composition of Stellite 21 and Stellite 720 [20].............................................. 31

Table 3-1: Chemical compositions (wt.\%) of 700 series Stellite alloys $[1,3]$........................ 49

Table 3-2: Carbide volume fraction of 700 series Stellite alloys [11] .................................. 50

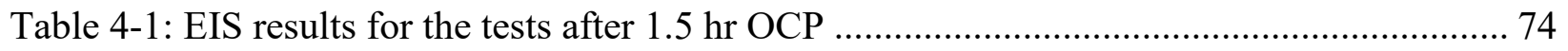


Table 4-2: Comparison of EIS results for different OCP durations at $25^{\circ} \mathrm{C}$ 78

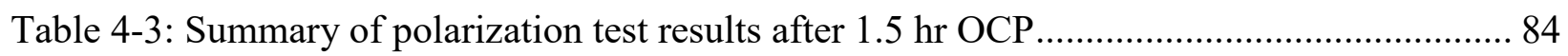

Table 4-4: Summary of polarization parameters for all OCP durations ................................ 87

Table 4-5: EDS elemental concentration (wt.\%) of corroded Stellite 706 surface..................... 96

Table 4-6: EDS elemental concentration (wt.\%) of corroded Stellite 712 surface..................... 97

Table 4-7: EDS elemental concentration (wt.\%) of corroded Stellite 720 surface..................... 98

Table 4-8: Valence values used in EW calculations ......................................................... 104

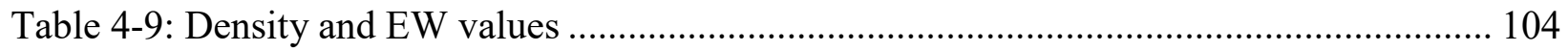

Table 4-10: Potentiostatic polarization results under constant potentials at $25^{\circ} \mathrm{C} \mathrm{\ldots ................} 104$

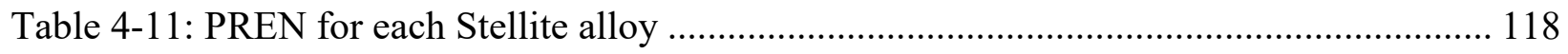

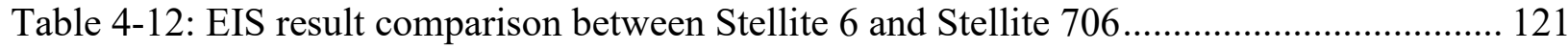

Table 4-13: Polarization result comparison between Stellite 6 and Stellite 706 ..................... 124

\section{List of Figures}

Figure 1-1: SEM microstructure of as-cast Stellite 6 [5] ....................................................... 2

Figure 1-2: Corrosion damaged Stellite 6 hard-faced valve seat and plug [5] ......................... 4

Figure 2-1: SEM microstructure of (a) Stellite 706, (b) Stellite 712 and (c) Stellite 720 [11] .... 11

Figure 2-2: XRD spectrum of (a) Stellite 706, (b) Stellite 712 and (c) Stellite 720 [11] ........... 12

Figure 2-3: Corrosion rates of Stellite 6, Stellite 12, Stellite 706, and Stellite 712 in acid

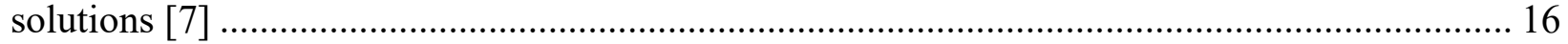

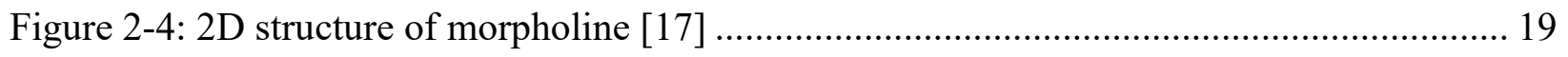

Figure 2-5: SEM microstructure of Stellite 6 (a) as-cast and (b) HIPed [18] ........................... 21

Figure 2-6: OCP curves for as-cast and HIPed Stellite 6 after $168 \mathrm{hr}$ exposure to $3.5 \mathrm{wt} . \% \mathrm{NaCl}$

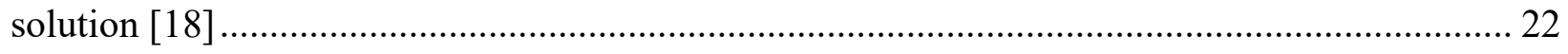


Figure 2-7: Potentiodynamic polarization curves of as-cast and HIPed Stellite 6 after $168 \mathrm{hr}$

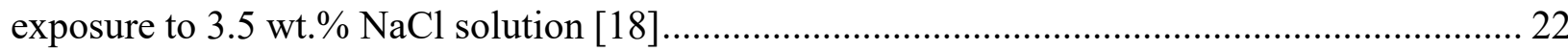

Figure 2-8: Cyclic polarization curve of (a) Stellite 6 and (b) 17-4PH stainless steel in

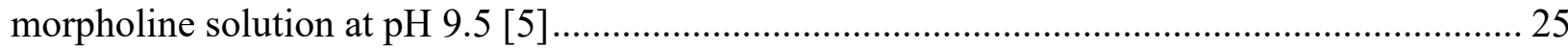

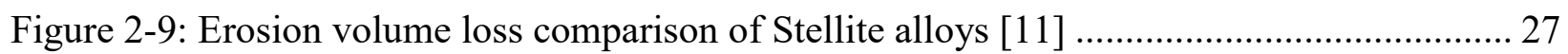

Figure 2-10: Polarization curves for Stellite 6B and Stellite $6 \mathrm{~K}$ in (a) $3.5 \mathrm{wt} . \% \mathrm{NaCl}$ solution and

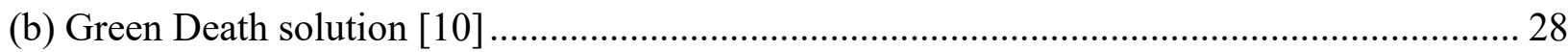

Figure 2-11: Bode plot of Stellite 6B tested in 3.5 wt.\% $\mathrm{NaCl}$ solution: (a) impedance and (b) phase angle [6]

Figure 2-12: Bode plot of Stellite $6 \mathrm{~K}$ tested in $3.5 \mathrm{wt} . \% \mathrm{NaCl}$ solution: (a) impedance and (b) phase angle [6] 30

Figure 2-13: Bode plot of Stellite alloys in Hanks solution: (a) impedance and (b) phase angle $[20]$ 32

Figure 2-14: XPS spectra of Fe-18Cr-14.3Ni-2.5Mo alloy [35] .......................................... 33

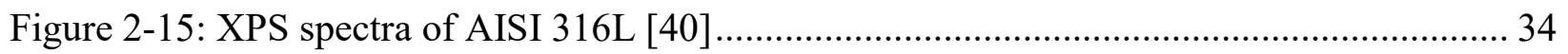

Figure 2-16: An example of a phase shift between the applied voltage and current response

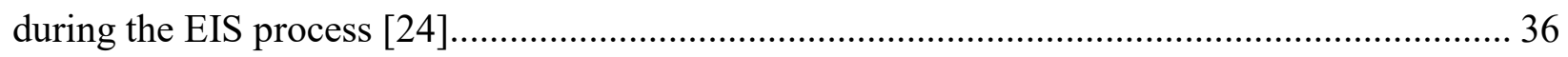

Figure 2-17: EIS spectra of (a) Bode plot and (b) Nyquist plot [24] ..................................... 38

Figure 2-18: Equivalent circuit model: (a) a pure capacitor and (b) Randles circuit for an

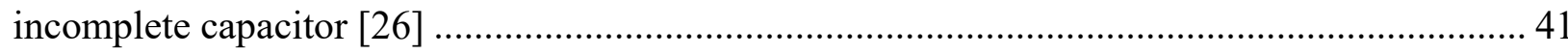

Figure 2-19: Schematic representation of the simplified Randles circuit [24] ........................ 42

Figure 2-20: An example of bode plot for the reference Randles circuit [26] ........................ 43

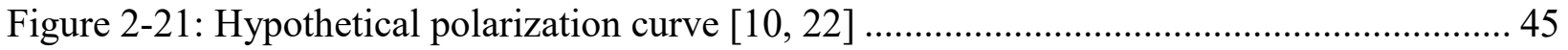


Figure 2-22: Cyclic polarization curves displaying hysteresis loop (dotted lines) [23] ............. 48

Figure 3-1: Tescan Vega-II XMU SEM instrument ........................................................... 50

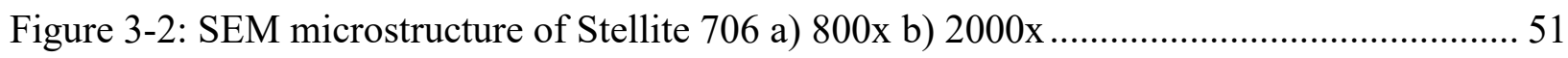

Figure 3-3: SEM microstructure of Stellite 712 a) 800x b) 2000x ......................................... 51

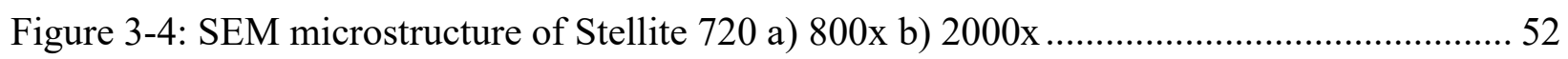

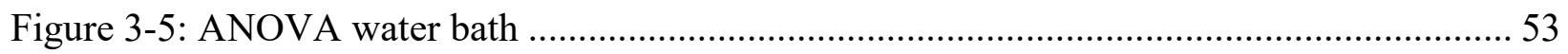

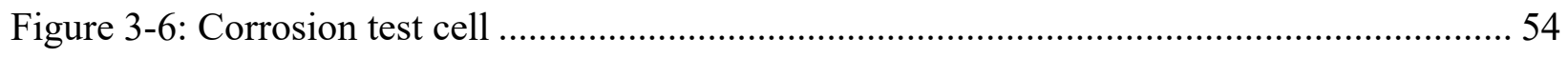

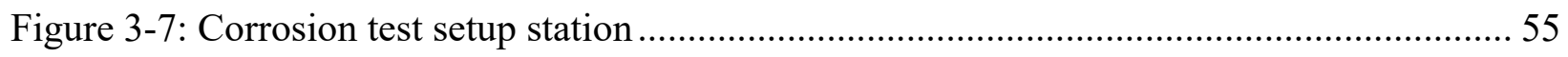

Figure 3-8: Ball-on-Disc Tribometer: (a) overall setup, (b) specimen holder and (c) photographic

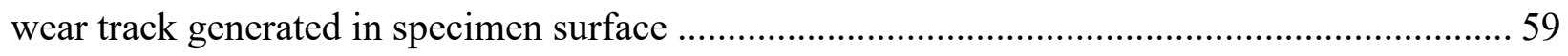

Figure 3-9: SEM images of worn surface (a) Stellite 706, (b) Stellite 712 and (c) Stellite $720 \ldots 60$

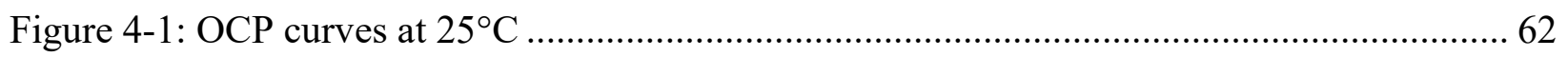

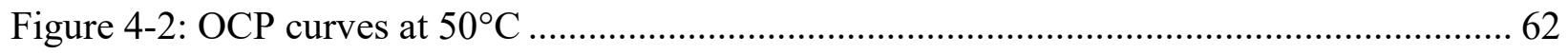

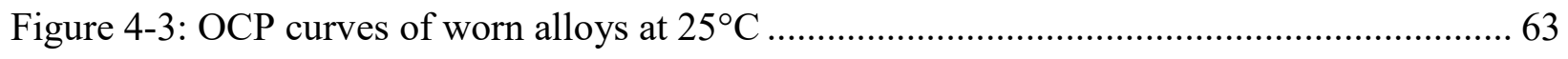

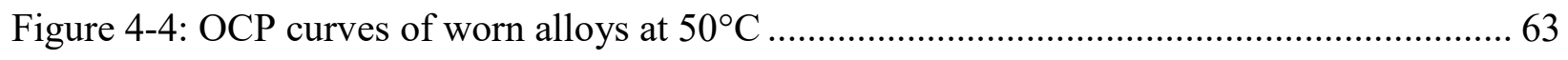

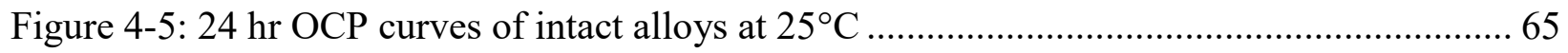

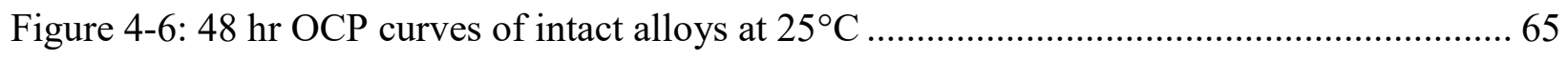

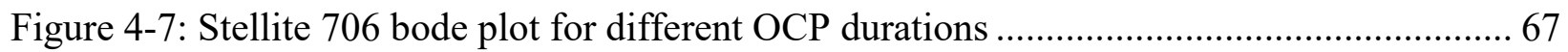

Figure 4-8: Morpholine solution resistance and Stellite 706 impedance curves for different OCP

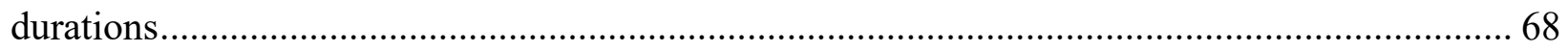

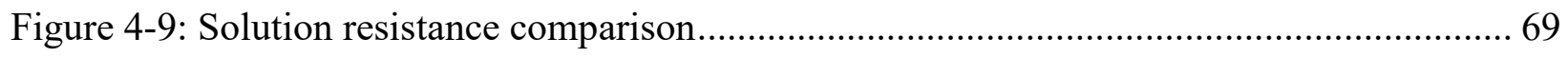

Figure 4-10: Impedance plots showing the effect of salt bridge not used ............................. 70

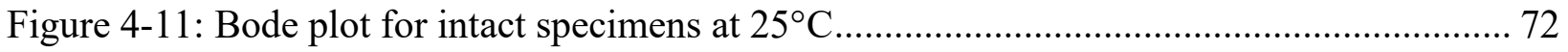


Figure 4-12: Bode plot for intact specimens at $50^{\circ} \mathrm{C}$. 72

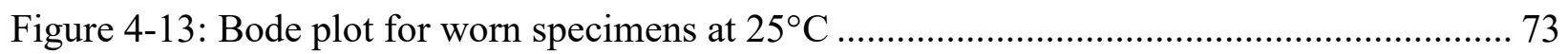

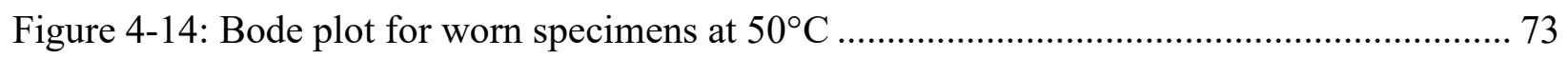

Figure 4-15: Bar graph representation of $R_{p}$ for intact specimens ..................................... 75

Figure 4-16: Bar graph representation of $R_{p}$ for worn specimens ..................................... 75

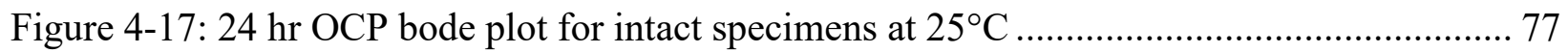

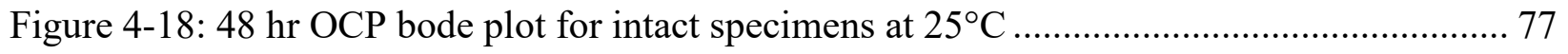

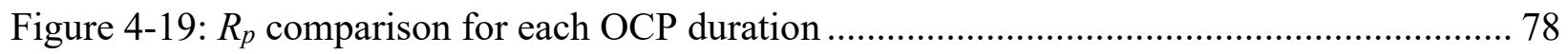

Figure 4-20: Initial equivalent circuit model for tested alloys............................................ 79

Figure 4-21: Final circuit model for tested alloys .......................................................... 79

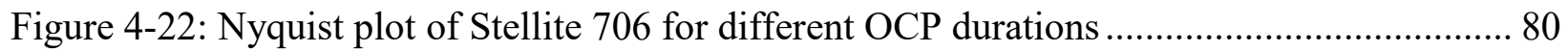

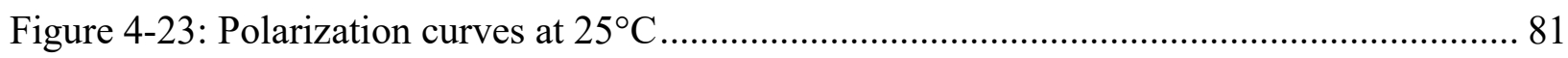

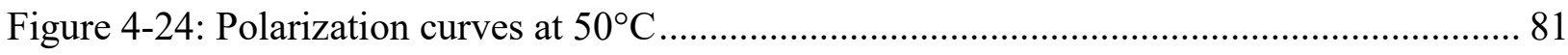

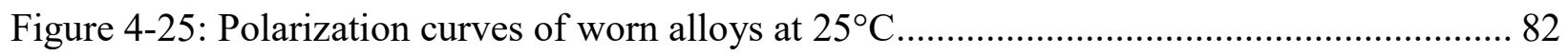

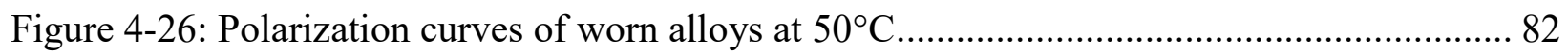

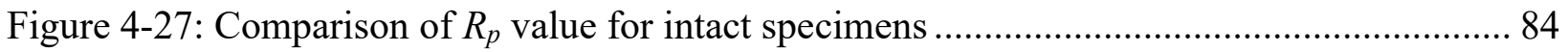

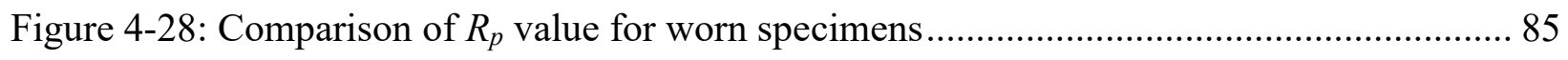

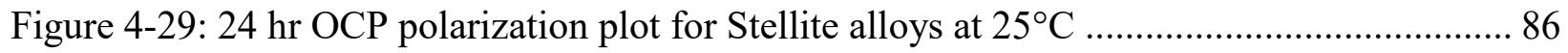



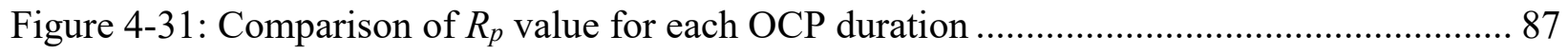

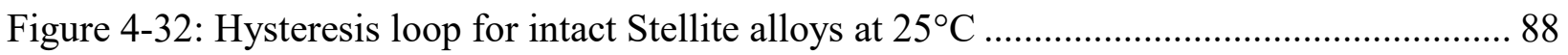

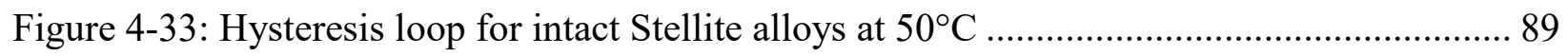

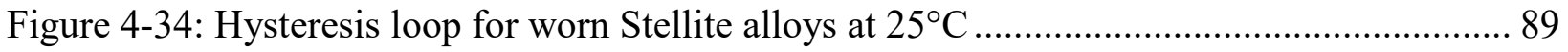


Figure 4-35: Hysteresis loop for worn Stellite alloys at $50^{\circ} \mathrm{C}$ 90

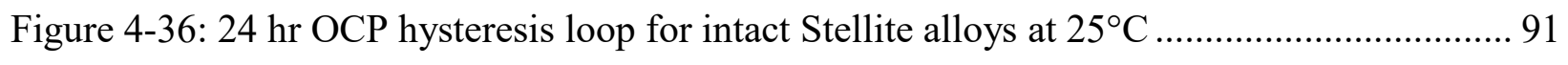

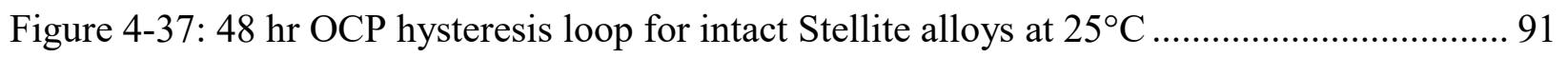

Figure 4-38: SEM images of Stellite 706: (a) corroded surface in morpholine solution with pH

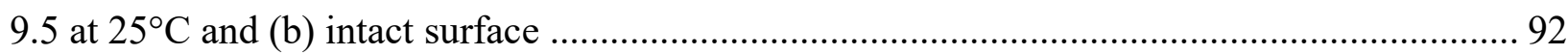

Figure 4-39: SEM images of Stellite 712: (a) corroded surface in morpholine solution with pH

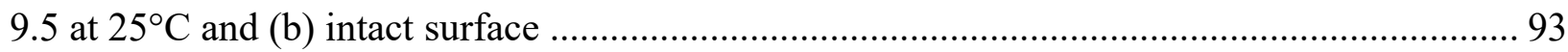

Figure 4-40: SEM images of Stellite 720: (a) corroded surface in morpholine solution with pH

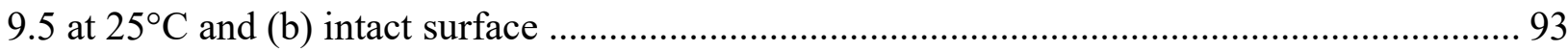

Figure 4-41: 4000x magnification image of Stellite 706 showing fractured oxide layer regions

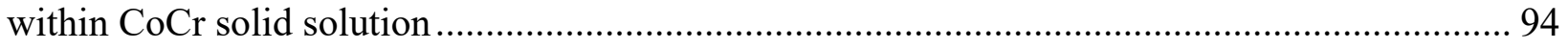

Figure 4-42: 4000x magnification image of Stellite 712 showing fractured oxide layer regions

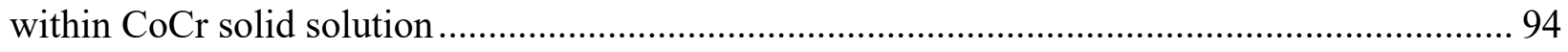

Figure 4-43: 4000x magnification image of Stellite 720 showing fractured oxide layer region on

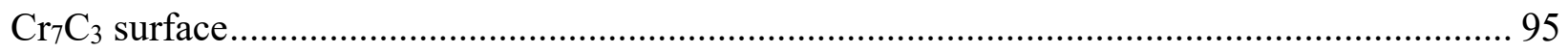

Figure 4-44: Stellite 706 surface after polarization test................................................. 96

Figure 4-45: Stellite 712 surface after polarization test................................................ 97

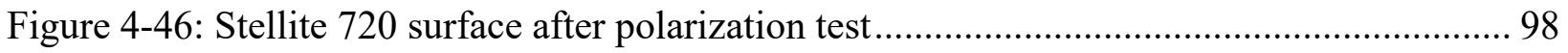

Figure 4-47: EDS spectra of Stellite 706: (a) Cr-rich region, (b) $\mathrm{Cr}$ and Co-rich region and (c)

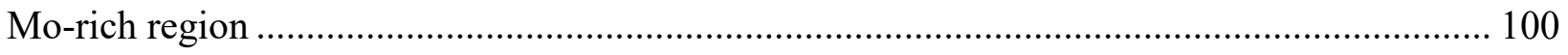

Figure 4-48: Current density variation for $1 \mathrm{~V}$ potential .............................................. 101

Figure 4-49: Current density variation for $2 \mathrm{~V}$ potential .................................................. 101

Figure 4-50: Current density variation for $3 \mathrm{~V}$ potential ................................................ 102 
Figure 4-51: Current variation for the condition of $3 \mathrm{~V}$ potential at $50^{\circ} \mathrm{C}$ 105

Figure 4-52: Trapezoid rule plot [46] 106

Figure 4-53: Current density variations at $1 \mathrm{~V}$ potential in $25^{\circ} \mathrm{C}$ morpholine solution with $\mathrm{pH} 9.5$ 107

Figure 4-54: Overall current densities at a constant potential in $25^{\circ} \mathrm{C}$ morpholine solution with $\mathrm{pH} 9.5$. 108

Figure 4-55: Overall current densities at a constant potential in $50^{\circ} \mathrm{C}$ morpholine solution with

$\mathrm{pH} 9.5$. 109

Figure 4-56: Cyclic polarization of Stellite 706 under peak voltage of $12 \mathrm{~V}$ in morpholine ..... 110

Figure 4-57: Cyclic polarization of Stellite 712 under peak voltage of $12 \mathrm{~V}$ in morpholine ..... 111

Figure 4-58: Cyclic polarization of Stellite 720 under peak voltage of $12 \mathrm{~V}$ in morpholine ..... 111

Figure 4-59: Stellite 706 surface after exposure of up to $12 \mathrm{~V}$ in morpholine 112

Figure 4-60: Cyclic polarization curves of (a) Stellite 706, (b) Stellite 712 and (c) Stellite 720 tested in 3.5 wt. $\% \mathrm{NaCl}$ under the peak voltage of $8 \mathrm{~V}$ 114

Figure 4-61: Stellite 706 surface corrosion in 3.5 wt.\% $\mathrm{NaCl}$ solution. 114

Figure 4-62: Impedance variations within the negative hysteresis range for (a) Stellite 706, (b)

Stellite 712 and (c) Stellite 720. 117

Figure 4-63: SEM microstructure of (a) Stellite 6 [5] and (b) Stellite 706. 119

Figure 4-64: EIS comparison for all OCP durations between (a) Stellite 6 and (b) Stellite 706121

Figure 4-65: Comparison of $R_{p}$ between Stellite 6 and Stellite 706 122

Figure 4-66: Polarization curves comparison between (a) Stellite 6 and (b) Stellite 706 123

Figure 4-67: Comparison of $R_{p}$ between Stellite 6 and Stellite 706 124

Figure 4-68: Hysteresis comparison between (a) Stellite 6 and (b) Stellite 706 125 
Figure 4-69: Cyclic polarization comparison between (a) Stellite 6 and (b) Stellite 706 in morpholine

Figure 4-70: Stellite 6 after $12 \mathrm{~V}$ failure test in morpholine.

Figure 4-71: Cyclic polarization comparison between (a) Stellite 6 and (b) Stellite 706 in 3.5

wt. $\% \mathrm{NaCl}$

Figure 4-72: Overall comparison of all Stellite alloys for (a) EIS and (b) polarization after 48 hours OCP in morpholine 130

Figure 4-73: Pourbaix diagram of Molybdenum-water system [38] 134

Figure 4-74: Pourbaix diagram for Chromium-water system [38] 135

Figure 4-75: Pourbaix diagram for Cobalt-water system [48] 135

Figure 5-1: Historical costs of $\mathrm{Co}, \mathrm{Cr}$ and $\mathrm{Mo}$ in the past six years 138

Figure 5-2: Historical costs of the Stellite alloys under study 139 


\section{List of Acronyms}

\begin{tabular}{ll} 
PM & Powder metallurgy \\
HIP & Hot isostatic pressing \\
FCC & Face centered cubic \\
HCP & Hexagonal close packed \\
SEM & Scanning electron microscopy \\
EDS & Energy dispersive spectroscopy \\
XRD & X-ray diffraction \\
XPS & X-ray photoelectron spectroscopy \\
SFE & Stacking fault energy \\
ASTM & American society for testing and material \\
EIS & Electrochemical impedance spectroscopy \\
OCP & Open circuit potential \\
WE & Working electrode \\
CE & Counter electrode \\
RE & Reference electrode \\
EW & Equivalent weight \\
CPE & Constant phase element \\
PREN & Pitting resistance equivalent number \\
SCE & Saturated calomel electrode \\
\hline &
\end{tabular}




\section{Chapter: Introduction}

\subsection{Background and Significance}

\subsubsection{Synopsis of Stellite alloys}

Stellite alloys are cobalt-based superalloys, designed mainly for wear and corrosion resistance and high-temperature applications. These alloys have been successfully used since World War One for hard facing in applications such as hot trimming dies, combustion engine valves and valves seats, plowshares, etc. [1]. During World War Two, further use of Co-Cr-Mo ternary alloys became an important part of dental and surgical tools due to their biocompatibility [1]. Stellite alloys have a unique combination of various properties such as cavitation and erosion resistance, abrasive and adhesive wear resistance, hardness, high strength, and good mechanical performance. Stellite alloys are now extensively used for their proven high-temperature wear and corrosion resistance in a broad range of industries such as automotive, power generation, glass, aerospace, petroleum, steel, pulp and paper, and other process industries [1].

Stellite alloy is typically identified as either high- or low-carbon alloy depending on its application. High-carbon Stellite alloys are designed for severe wear services, while low-carbon Stellite alloys are designed for high-temperature wear and corrosion resistance [1]. Chemical compositions of Stellite alloys are mainly composed of cobalt $(\mathrm{Co})$ in balance, chromium $(\mathrm{Cr})$, carbon $(\mathrm{C})$, molybdenum (Mo) and/or tungsten (W). $\mathrm{Cr}$ is the predominant carbide former and the main element that provides corrosion resistance. Mo and $\mathrm{W}$ improve general corrosion resistance and provide additional strength to the matrix [1].

An important factor that influences the microstructure of Stellite alloys and their properties is the fabrication process. Stellite $6(29 \% \mathrm{Cr}, 4.5 \% \mathrm{~W}, 1.5 \% \mathrm{Mo}, 1.2 \% \mathrm{C}$ in weight) for example is one of the common Stellite alloys known in the Stellite family and its microstructure in as-cast form 
typically displays a eutectic consisting of wear-resistant carbides and cobalt-chromium $(\mathrm{CoCr})$ ductile matrix as shown in Figure 1-1. The major Cr-rich carbides (in dark) and minor W-rich carbides (in light) provide abrasion and sliding wear resistance, while the matrix provides toughness [2].

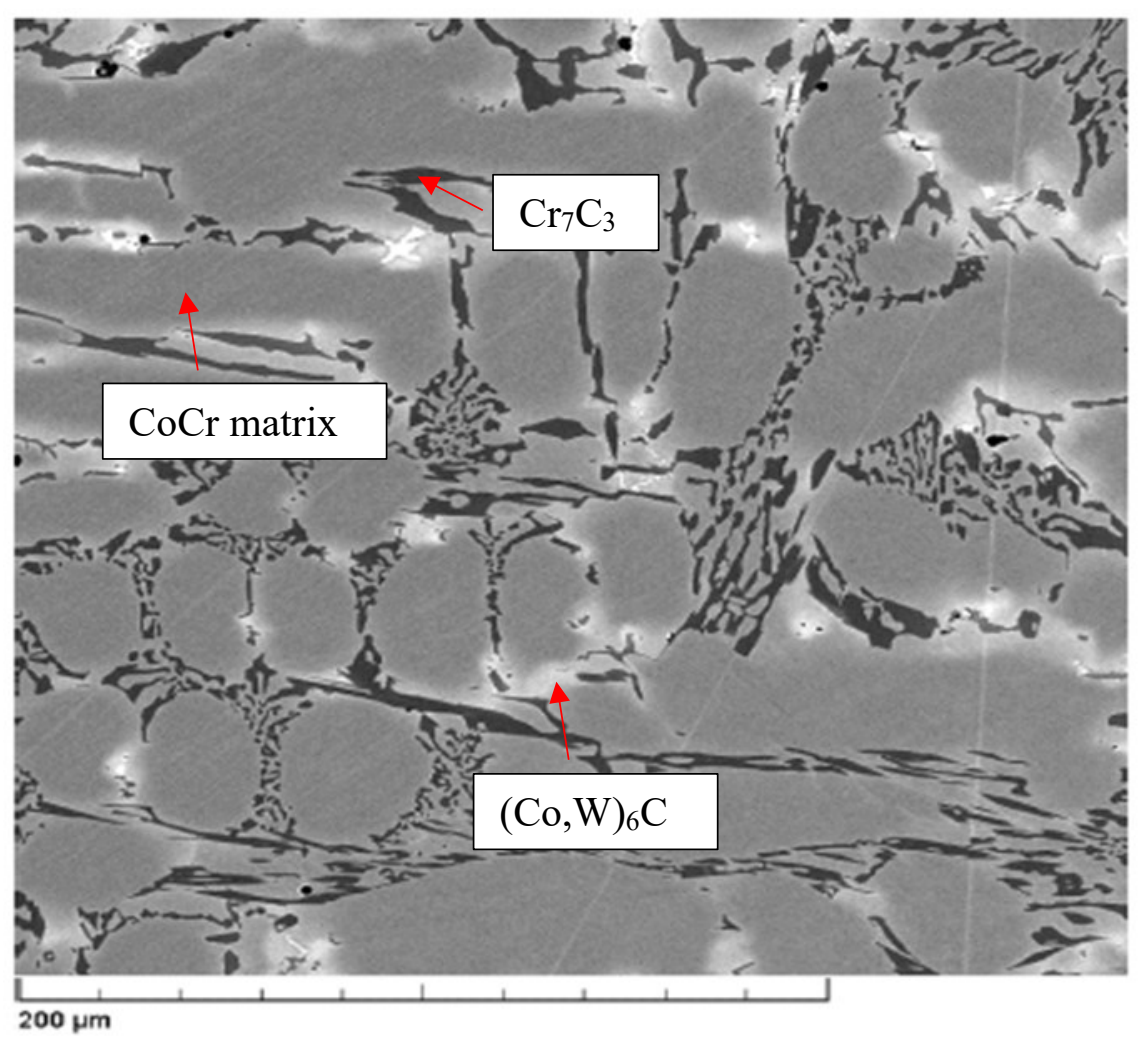

Figure 1-1: SEM microstructure of as-cast Stellite 6 [5]

Cast 700 series Stellite alloys such as Stellite 706, Stellite 712 and Stellite 720 all contain high C ( $\geq 1.2$ wt.\%) and high Mo ( $\geq 5$ wt.\%) contents. The difference among them is their $\mathrm{C}$ and Mo content. These alloys display similar microstructure characteristics such as hard carbides embedded in tough $\mathrm{CoCr}$ solid solution matrix. 700 series Stellite alloys use $\mathrm{Cr}$ and $\mathrm{Mo}$ as major alloying elements and can offer both excellent wear and corrosion resistance when in contact with reducing aqueous solutions such as phosphoric and hydrochloric acids [3]. 


\subsubsection{Corrosion problems}

Corrosion is a process which metals and alloys deteriorate over time, degrading in their properties and leading to eventual failure. It is a problem no matter the application because it is practically impossible to prevent corrosion. However, it can be managed, and the service life of the materials can be prolonged safely before eventual failure. Therefore, research and development of high corrosion-resistant alloys are important for industries such as oil and gas production. Stellite alloys are generally corrosion resistant due to their high $\mathrm{Cr}$ content, however because of major alloying elements present they are multiphase alloys. Having multiple phases means more interfaces in the matrices, which results in more grain boundaries that are high-energy sites for corrosion. With increased grain boundaries, corrosion resistance to aqueous solution can be limited. Thus, if C content is low in Stellite alloys ( $\sim 0.025$ to 0.15 wt.\%) the alloys are much better for severe corrosion resistance at high temperatures where ductility is an important factor along with less second phase particles. With reduced $\mathrm{C}$ in the composition, there are more $\mathrm{CoCr}$ solid solution and less carbide intermetallic, thus effectively reducing interfaces, whereas with high $\mathrm{C}$ content (1.2 to 3 wt.\%) there are more interfaces and less ductility, but the alloy has better wear resistance.

In industrial applications that involve high temperature, there is always synergetic attack of wear and corrosion where Stellite alloys are required to combat such environments. These alloys can be used for feedwater regulator valves as hardfacing of the valve trim components. However, the alloys themselves have been criticized for poor performance when boiler feedwaters contain hydrazine $\left(\mathrm{N}_{2} \mathrm{H}_{4}\right)$ and other types of amine derivatives [4]. It is theorized by Kennametal Stellite Inc. that amine compounds weaken the $\mathrm{Cr}$ oxide layer allowing accelerated corrosion when the flow velocity of the feedwater reaches around $10 \mathrm{~m} / \mathrm{s}$ with an operating temperature of $150^{\circ} \mathrm{C}$. An 
example shown in Figure 1-2 is an eroded valve seat hard-faced with Stellite 6 that failed in service [5], but this type of failure has not been studied for many years.

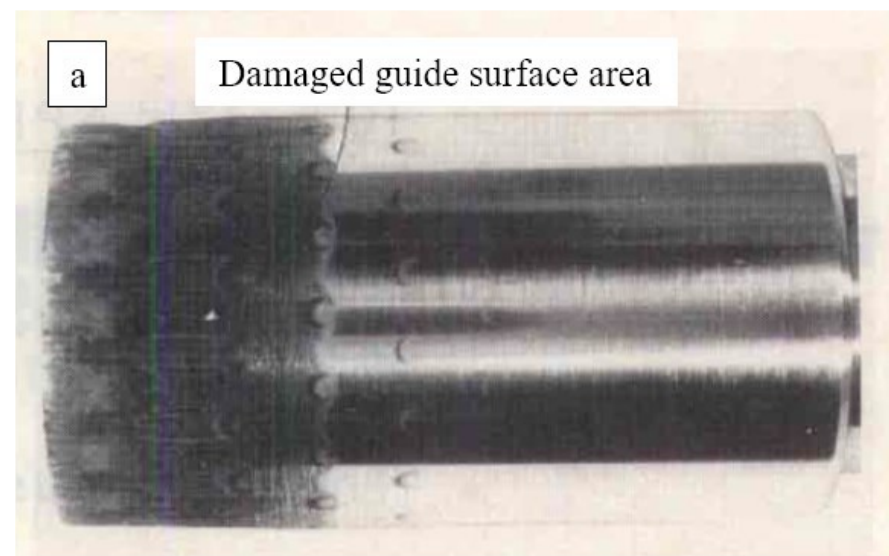

a. Erosion/corrosion damage of valve plug occurred at seat and well along the plug

b. Seat end of plug had damage band next to outer diameter. Cut was for specimen removal

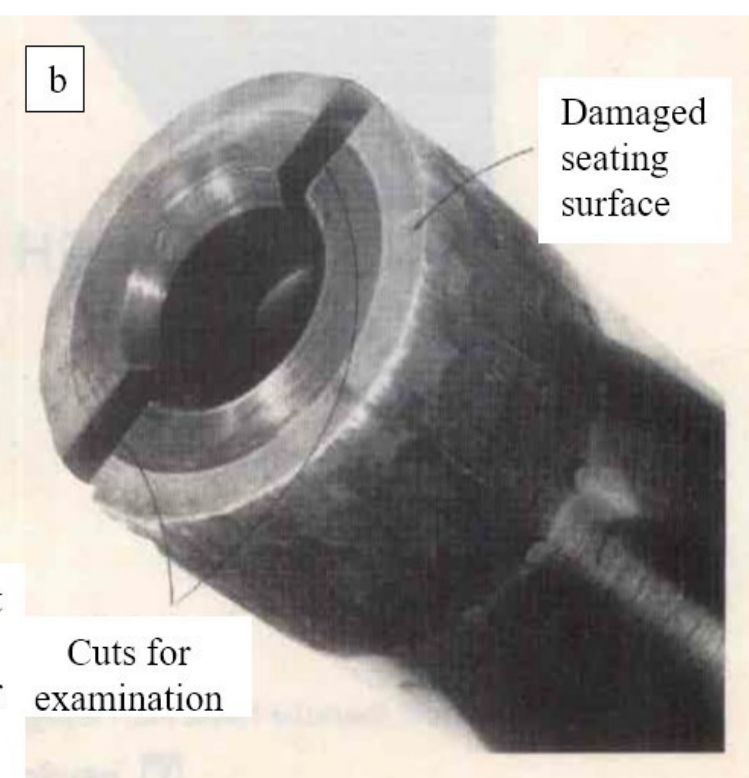

Figure 1-2: Corrosion damaged Stellite 6 hard-faced valve seat and plug [5]

Under normal operating conditions, boiler feedwater has been considered non-corrosive to Stellite alloys since Stellite 6 has been the primary alloy for hardfacing on valve trims in such environment. However, in the past decade, it had been reported by Kennametal Stellite Inc. that Stellite 6 had gone through multiple failures in service on feedwater control valves in cogeneration and electric power utility plants where hydrazine and/or amine derivatives are used. It is not understood why this failure occurs on Stellite alloys, but not on the alternative austenitic stainless steels, which have limited wear resistance. This has restricted the use of Stellite alloys in boiler feedwater treatment systems. Research on Stellite alloys for corrosion in $3.5 \mathrm{wt} . \% \mathrm{NaCl}$ and in various acids such as Green Death solution (primarily sulfuric acid), nitric acid, hydrochloric acid [6-8] has been extensively done, but rarely in basic solutions. In the case of boiler feedwater, the cleaning reagents use amine derivatives as a means of hydrophobic film formation [9] on metal surfaces to create an 
extra barrier for inhibiting charge transfer in addition to maintaining alkalinity. However, there is a lack of data for corrosion behaviour of Stellite alloys in amine solutions.

\subsection{Present Research}

\subsubsection{Objectives}

The thesis research is an attempt to understand the corrosion behaviour of three different types of Stellite alloys, Stellite 706, Stellite 712 and Stellite 720, which contain high C and high Mo. These alloys are relatively new compared to Stellite 6 , designed for improved corrosion resistance due to increased Mo content and are candidates for replacing Stellite 6 for valve trim component hardfacing in boiler feedwater treatment systems. These Stellite alloys provided by Kennametal Stellite Inc. for this research are as-cast products. Morpholine is primarily used in this research as the electrolyte solution to create an amine environment for corrosion studies using electrochemical testing methods such as electrochemical impedance spectroscopy (EIS), potentiodynamic polarization and potentiostatic polarization. The testing methods are employed on intact and damaged surface conditions under $25^{\circ} \mathrm{C}$ and $50^{\circ} \mathrm{C}$. The results obtained can then be compared to that of Stellite 6 alloy which is currently used in the application for valve trim hardfacing.

\subsubsection{Methodologies and tasks}

Three 700 series Stellite alloys (Stellite 706, Stellite 712 and Stellite 720) under study have similar chemical compositions with different amounts of major alloying elements. Stellite 706 contains 1.2 wt.\% C, Stellite 712 contains 2 wt.\% and Stellite 720 contains 2.5 wt.\%, which are classified as high C Stellite alloys. Stellite 706 and Stellite 712 contain the same amount of Cr (29 wt.\%) apart from Stellite 720 having 33 wt.\%. Mo content also varies amongst them where Stellite 706 has 5 wt.\%, Stellite 712 has 8.5 wt.\% and Stellite 720 has 18 wt.\%. Stellite 6 has 4.5 wt.\% W and 1.5 wt. $\%$ Mo. 
Multiple tests are conducted for consistency/reproducibility to quantify corrosion performance of these alloys and the tasks are outlined below:

1. Open circuit potential (OCP) testing is done initially as a minimum standard at 1.5 hours to stabilize the electrochemical testing system and obtain data to determine the starting point of the polarization test. Morpholine based electrolyte is used for all tests with a $\mathrm{pH}$ level of 9.5 at room temperature $\left(25^{\circ} \mathrm{C}\right)$ and at an elevated temperature of $50^{\circ} \mathrm{C}$.

2. EIS is utilized to study the integrity of the oxide film that forms on the intact alloy surface under the same OCP and temperature conditions.

3. Potentiodynamic polarization is utilized to study the general and localized corrosion behaviour under the same OCP and temperature conditions.

4. To investigate the influence of specimen surface condition on corrosion behaviour, Stellite alloy surfaces are damaged using a ball-on-disk tribometer, creating dry-sliding wear. Steps 2 and 3 are repeated.

5. Step 2 and 3 are repeated for 24 and 48 hours of OCP duration for intact specimens in $25^{\circ} \mathrm{C}$ only.

6. The surface morphology of each alloy after corrosion is examined using Scanning Electron Microscopy (SEM) and the elements of each phase are identified using Electron Dispersive Spectroscopy (EDS).

7. Failure test using potentiostatic testing is conducted to study the alloy behaviour at different constant potentials $(1 \mathrm{~V}, 2 \mathrm{~V}$ and $3 \mathrm{~V})$ for one hour in the same electrolyte medium at both temperature conditions for 1.5 hours OCP only.

8. Failure tests are also done using potentiodynamic polarization at higher peak voltages in morpholine and sodium chloride $(\mathrm{NaCl})$ solution to test the alloy limitation. 
9. Comparison of corrosion performance in morpholine solution with $\mathrm{pH} 9.5$ is made among the 700 series Stellite alloys and Stellite 6.

\subsection{Organization of Thesis}

This thesis is grouped into five chapters, beginning with Introduction (Chapter 1), which describes the background and significance (Section 1.1) of this research, (Section 1.2) then indicates the objectives of this research, methodologies used, and the tasks completed, and finally gives the organization of the thesis (Section 1.3).

Literature Review (Chapter 2) includes the overview and applications of Stellite alloys in the industry (Section 2.1), benefits of using amine (Section 2.2), and summarizes the previous researches that have been done in pertaining to corrosion studies on Stellite alloys (Section 2.3). Methods of corrosion study (Section 2.4) reviews different methods that can be used for corrosion study including EIS, potentiodynamic and potentiostatic polarization.

Experimental Details (Chapter 3) cover the alloys in the study (Section 3.1), solution preparation (Section 3.2), experimental setup (Section 3.3), experimental procedure (Section 3.4), and ball-ondisc wear test (Section 3.5).

Results and Discussion (Chapter 4) include the experimental results from OCP (Section 4.1), EIS (Section 4.2), polarization (Section 4.3), surface morphology of corroded specimens (Section 4.4), failure tests (Section 4.5), comparison with Stellite 6 (Section 4.6), and ending with discussion on these results and analyses (Section 4.7).

Conclusions and Future Work (Chapter 5) provide a summary (Section 5.1) of this research work and then highlights the main conclusions (Section 5.2), research implications (Section 5.3), and suggested future works (Section 5.4). 


\section{Chapter: Literature Review}

\subsection{Overview of Stellite Alloys}

\subsubsection{Chemical composition and microstructure}

Two main groups of Stellite alloys are $\mathrm{CoCr}$ with $\mathrm{W}$ and $\mathrm{CoCr}$ with $\mathrm{Mo}$. The chemical compositions of various Stellite alloys are composed primarily of Co as balance (approximately 40 - 60 wt.\%), 20 - 33 wt.\% Cr, 5 - 18 wt.\% of W or Mo, and 0.02 - 3 wt.\% C [1]. Table 2-1 summarizes different types of Stellite alloys and their compositions. The main difference among the alloys is noted in the amount of W/Mo and C added to the system, which dictates carbide formation during solidification.

Table 2-1: Chemical compositions (wt.\%) of Stellite alloys [1, 10]

\begin{tabular}{|c|c|c|c|c|c|c|c|c|c|c|}
\hline Alloy & Process & Cr & W & Mo & C & Fe & Ni & Si & Mn & Others \\
\hline Stellite 1 & Cast & 30 & 13 & 0.5 & 2.5 & 3 & 1.5 & 1.3 & 0.5 & \\
\hline Stellite 3 & PM & 30.5 & 12.5 & & 2.4 & 5 & 3.5 & 2 & 2 & $1 \mathrm{~B}$ \\
\hline Stellite 4 & Cast & 30 & 14 & 1 & 0.57 & 3 & 3 & 2 & 1 & \\
\hline Stellite 6 & Cast/PM & 29 & 4.5 & 1.5 & 1.2 & 3 & 3 & 1.5 & 1 & $1 \mathrm{~B}(\mathrm{PM})$ \\
\hline Stellite 12 & Cast & 30 & 8.3 & & 1.4 & 3 & 1.5 & 0.7 & 2.5 & \\
\hline Stellite 21 & Cast & 27 & & 5.5 & 0.25 & 3 & 2.75 & 1 & 1 & \\
\hline Stellite 20 & Cast & 33 & 17.5 & & 2.45 & 2.5 & 2.5 & & 1 & \\
\hline Stellite 22 & Cast & 27 & & 11 & 0.25 & 3 & 2.75 & 1 & 1 & \\
\hline Stellite 28 & Cast & 24 & & 12 & 0.35 & & 4 & & & $2 \mathrm{Nb}$ \\
\hline Stellite 25 & Cast & 20 & 15 & & 0.1 & 3 & 10 & 1 & 1.5 & \\
\hline Stellite 31 & Cast & 22 & 7.5 & & 0.5 & 1.5 & 10 & 0.5 & 0.5 & \\
\hline Stellite 80 & Cast & 33.5 & 19 & & 1.9 & & & & & $1 \mathrm{~B}$ \\
\hline Stellite 188 & Cast & 22 & 14 & & 0.1 & 3 & 22 & 0.35 & 1.25 & $0.03 \mathrm{La}$ \\
\hline Stellite 190 & Weld & 27 & 14 & 1 & 3.3 & 3 & 3 & 1 & 1 & \\
\hline Stellite 300 & Cast & 22 & 32 & & 1.5 & & & & & \\
\hline
\end{tabular}




\begin{tabular}{|c|c|c|c|c|c|c|c|c|c|c|}
\hline Stellite 694 & Cast & 28 & 19 & & 1 & 5 & & 1 & 1 & \\
\hline Stellite 703 & Cast & 32 & & 12 & 2.4 & 3 & 3 & 1.5 & 1.5 & \\
\hline Stellite 706 & Cast & 29 & & 5 & 1.2 & 3 & 3 & 1.5 & 1.5 & \\
\hline Stellite 712 & Cast & 29 & & 8.5 & 2 & 3 & 3 & 1.5 & 1.5 & \\
\hline Stellite 720 & Cast & 33 & & 18 & 2.5 & 3 & 3 & 1.5 & 1.5 & $0.3 \mathrm{~B}$ \\
\hline Stellite F & Cast & 25 & 12.3 & 1 & 1.75 & 3 & 22 & 2 & 1 & \\
\hline $\begin{array}{c}\text { Stellite Star } \\
\text { J }\end{array}$ & Cast/PM & 32.5 & 17.5 & & 2.5 & 3 & 2.5 & 2 & 2 & $1 \mathrm{~B}(\mathrm{PM})$ \\
\hline Stellite 706K & Wrought & 29 & & 6 & 1.4 & 3 & 3 & 1.5 & 1.5 & \\
\hline Stellite 6B & Wrought & 30 & 4 & 1.5 & 1 & 3 & 2.5 & 0.7 & 1.4 & \\
\hline Stellite 6K & Wrought & 30 & 4.5 & 1.5 & 1.6 & 3 & 3 & 2 & 2 & \\
\hline $\begin{array}{c}\text { Stellite } \\
\text { 98M2 }\end{array}$ & PM & 30 & 18.5 & 0.8 & 2 & 5 & 3.5 & 1 & 1 & $4.2 \mathrm{~V}$, \\
\hline
\end{tabular}

PM - Powder Metallurgy

Chemical composition and processing method dictate the microstructure of Stellite alloys. As seen in Figure 2-1 [11], the microstructures of Stellite 706, Stellite 712, and Stellite 720 all consist of Cr-rich (in dark) and Mo-rich (in light) carbides embedded in the $\mathrm{CoCr}$ solid solution matrix (in grey). The correlation between chemical composition and microstructure of Stellite alloys can be seen from Table 2-1 as the $\mathrm{C}$ content increases, the formation of more carbides is promoted. As the Mo content increases, so does the amount of Mo-rich carbides precipitated in the matrix. 


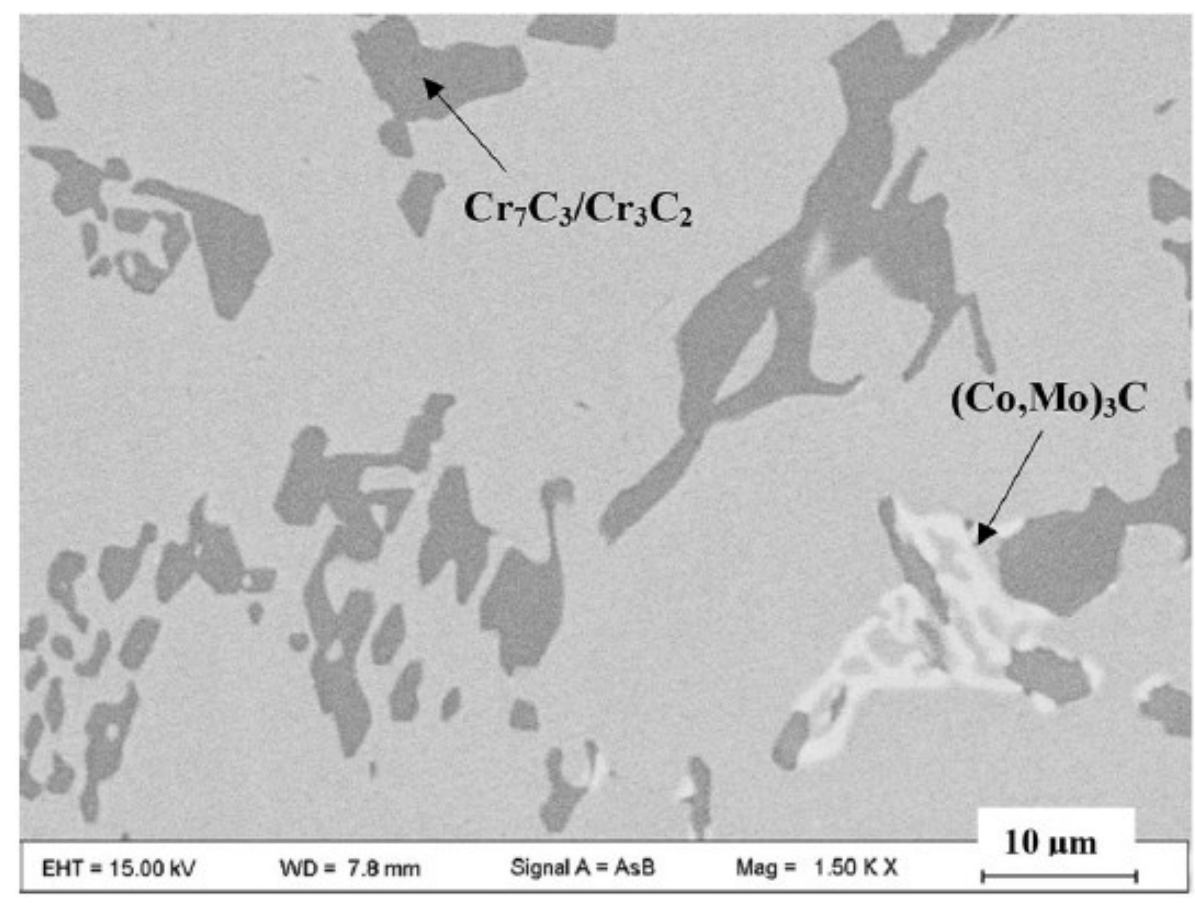

(a)

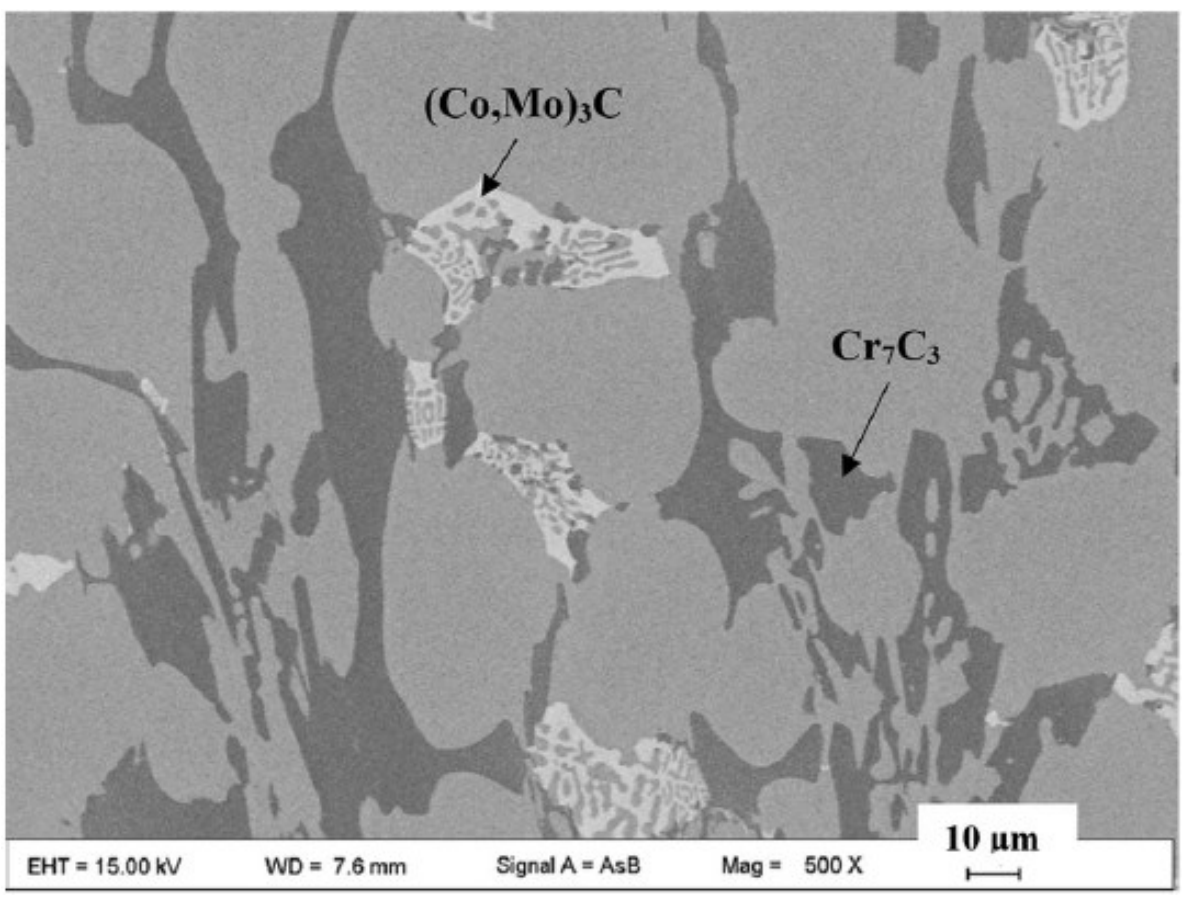

(b) 


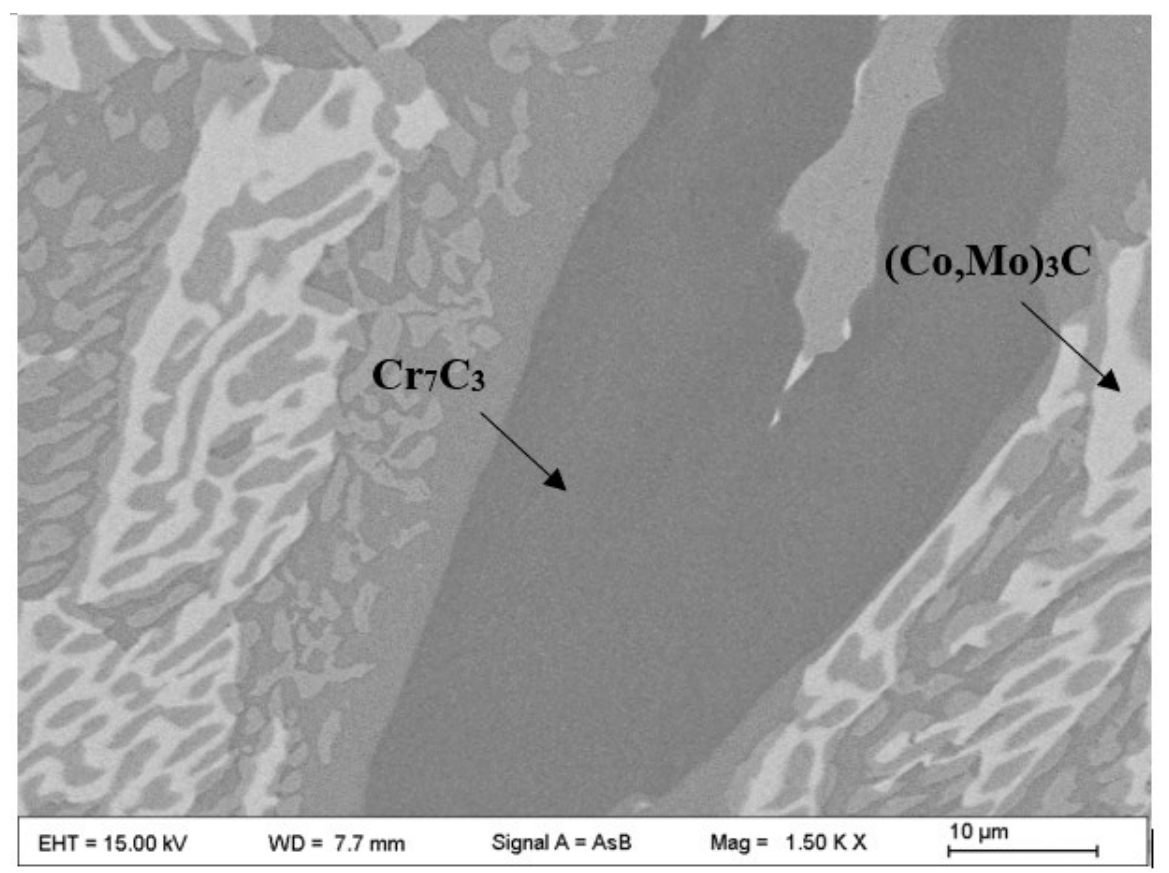

(c)

Figure 2-1: SEM microstructure of (a) Stellite 706, (b) Stellite 712 and (c) Stellite 720 [11]

The solid solution phase and various carbide phases in the alloy have been identified using X-ray diffraction (XRD) and the results are presented in Figure 2-2 [11]. It is demonstrated that the dark Cr-rich carbide is $\mathrm{Cr}_{7} \mathrm{C}_{3}$. Since Mo content is small in Stellite 706 (5 wt.\%), the Mo-rich carbide is not detected. Stellite 712 and 720 have a higher content of Mo, hence the XRD can detect the Mo-rich carbide phase, showing that it is $(\mathrm{Co}, \mathrm{Mo})_{3} \mathrm{C}$. The $\mathrm{CoCr}$ solid solution is a face centered cubic (fcc) matrix. 


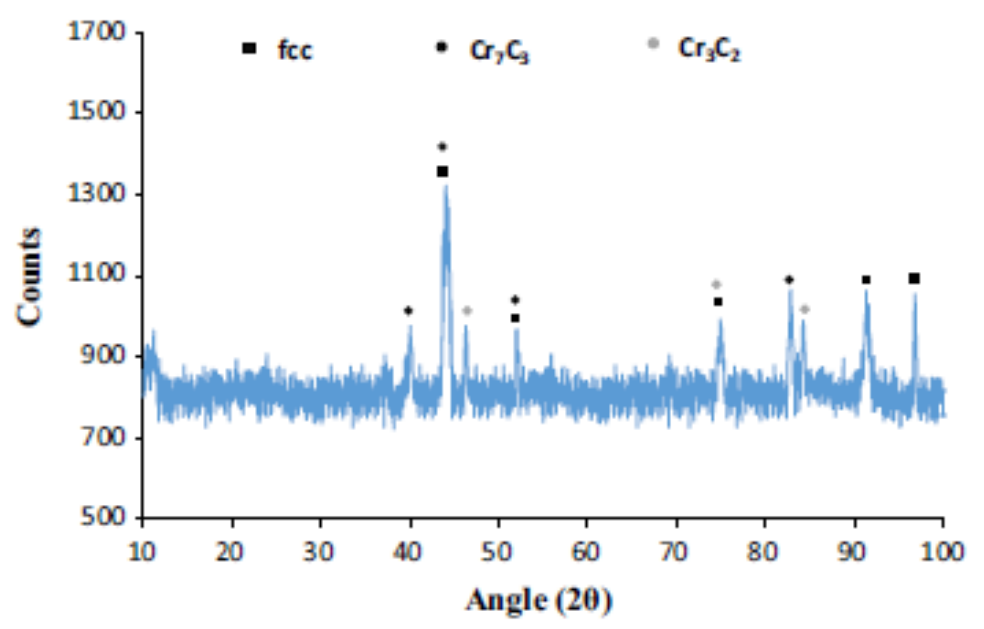

(a)

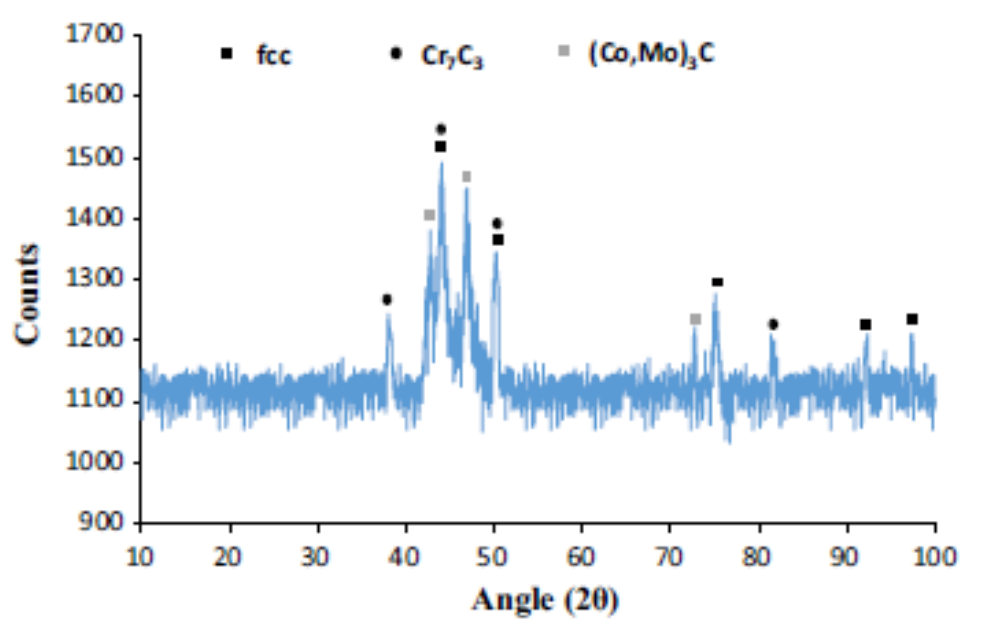

(b)

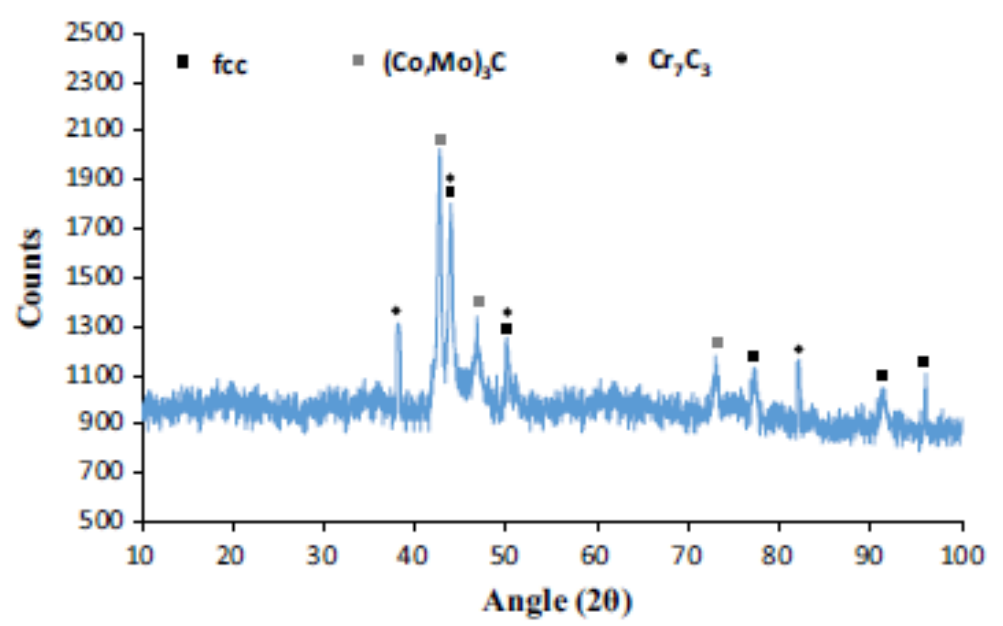

(c)

Figure 2-2: XRD spectrum of (a) Stellite 706, (b) Stellite 712 and (c) Stellite 720 [11] 


\subsubsection{Effects of alloying elements}

Stellite alloys contain multiple major alloying elements that make up the bulk of the microstructure such as $\mathrm{Cr}, \mathrm{C}, \mathrm{Mo}$, and/or W. Other residual elements within the composition include iron (Fe), nickel (Ni), silicon (Si), manganese $(\mathrm{Mn})$, tantalum $(\mathrm{Ta})$, niobium $(\mathrm{Nb})$, etc. depending on the intended application the alloy [1]. Each of the major alloying elements contributes to specific properties it provides such as phase stability at elevated temperatures; corrosion and wear resistance; fatigue and creep strength; and high mechanical strength/hardness [2]. Stellite alloys can be further broken down in detail in terms of their alloying elements to review their effects.

\subsubsection{Influence of carbon}

Depending on the amount of $\mathrm{C}$ added, different forms of carbide constituents are formed. Stellite alloys are typically divided into three categories, which are low $\mathrm{C}(<0.5 \mathrm{wt} \%$ ) for hightemperature service; medium C (0.5 - 1.2 wt.\%) for simultaneous corrosion and wear; and high C (1.2 - 3 wt.\%) for better wear performance [1]. The effect of adding $\mathrm{C}$ is for the formation of carbides such as $\mathrm{MC}, \mathrm{M}_{7} \mathrm{C}_{3}, \mathrm{M}_{23} \mathrm{C}_{6}, \mathrm{M}_{6} \mathrm{C}$, or $\mathrm{M}_{3} \mathrm{C}$, therefore $\mathrm{C}$ content in Stellite alloys determine the carbide volume fraction of their microstructures. For 700 series Stellite alloys, the main carbides are $\mathrm{M}_{7} \mathrm{C}_{3}$ where $\mathrm{M}$ represents $\mathrm{Cr}$ and $\mathrm{M}_{3} \mathrm{C}$ where $\mathrm{M}$ stands for Mo. The three Stellite alloys being studied are all categorized as high-C Stellite alloys thus ruling out $\mathrm{M}_{23} \mathrm{C}_{6}$ carbide formation since that is usually found in low-C Stellite alloys and $\mathrm{MC}$ is not considered due to the composition not having any titanium (Ti), $\mathrm{Ta}$ or $\mathrm{Nb}$ [1]. Having additional $\mathrm{C}$ can be a benefit for wear resistance, however, it can also be a detriment in Stellite alloys with respect to corrosion resistance due to more interphases. 


\subsubsection{Influence of chromium}

$\mathrm{Cr}$ has dual functionality in Stellite alloys where it provides the bulk of the oxidation resistance and is the predominant carbide former. The most common carbides in Stellite alloys is Cr-rich $\left(\mathrm{Cr}_{7} \mathrm{C}_{3}\right.$ and $\left.\mathrm{Cr}_{23} \mathrm{C}_{6}\right)$ carbides. $\mathrm{Cr}$ in Stellite alloys ranges from $20-33$ wt.\% and provides a wide range of passivity in different potential values. It is also a solution strengthening element as a solute [1].

\subsubsection{Influence of molybdenum}

Mo is the primary solid solution strengthener due to its respective atomic size, which impedes dislocation movement [1]. Mo with an atomic weight of $95.95 \mathrm{~g}$ is almost two times less compared to $\mathrm{W}(183.84 \mathrm{~g})$, which implies that there will be twice as many atoms in a matrix at the same weight percent. As mentioned above, secondary carbides such as $\mathrm{M}_{3} \mathrm{C}$ are formed depending on the solubility limit of Mo in Co [8]. If large quantities of Mo are added ( $>5 \mathrm{wt} . \%$ ), they participate in the formation of Mo-rich carbides such as $\mathrm{Mo}{ }_{3} \mathrm{C}$ and intermetallic compounds such as $\mathrm{Co}_{3} \mathrm{Mo}$ $[8,10]$. Additional Mo improves the general and localized corrosion resistance of Stellite alloys within the active regime, such as grain boundaries, pitting zones and cracked oxide areas [1, 3537, 39-41]. With the content of Mo up to $28 \mathrm{wt} \%$, Stellite alloys exhibit significantly improved resistance to non-oxidized acids [32].

\subsubsection{Influence of tungsten}

W has a similar function to Mo as a solid solution strengthener in Stellite alloys, serving to provide additional strength to the matrix by impeding dislocation flow. When present in large quantities, for example, in Stellite 1, it participates in the formation of carbides during alloy solidification and promotes the precipitation of $\mathrm{M}_{6} \mathrm{C}$. It also improves the general corrosion resistance of the alloys [32]. 


\subsubsection{Influence of cobalt}

The most critical reason for the usefulness of Stellite alloys is the Co balance. Co by itself as an element is unstable with an fcc crystal structure with low stacking fault energy [1]. The instability allows fcc to transform to hexagonal close-packed (hcp) structure at $417^{\circ} \mathrm{C}$ when cooled extremely slowly [1]. The fcc structure instability and low SFE result in high work hardening rate and yield strength, low fatigue damage under cyclic loading, and ability to absorb stresses (transformation to hcp) [1]. The first three attributes can help prevent mechanical damage in sliding wear and last two attributes can help enhance erosion-corrosion resistance [1].

\subsubsection{Other elements}

Other small additions of elements which include $\mathrm{Fe}, \mathrm{Mn}, \mathrm{Ni}, \mathrm{Si}, \mathrm{Nb}$, Ta, etc. depending on the application. Most commonly in Stellite alloys, the ranges of Fe, Ni, Si, and Mn are shown in Table 2-1. Fe and Ni are both added as solid solution strengtheners and to stabilize the fcc matrix [10]. $\mathrm{Si}$ is added primarily for deoxidation along with $\mathrm{Mn}$, but $\mathrm{Mn}$ can also increase hardenability as well as tensile strength [10].

\subsubsection{Corrosion properties of Stellite alloys}

As seen in Table 2-1 from the compositions of every Stellite alloy, it can be inferred that these alloys are generally corrosion-resistant with the large amounts of $\mathrm{Cr}$ present. Compositions with less $\mathrm{C}$ tends to be single phase alloys meaning that the matrix lacks interfaces, which is required for better corrosion resistance. Yao et al. [7] investigated Stellite alloys in aggressive acidic environments such as $5 \%$ hydrochloric acid $(\mathrm{HCl})$ at $40^{\circ} \mathrm{C}, 10 \%$ sulphuric acid $\left(\mathrm{H}_{2} \mathrm{SO}_{4}\right)$ at $66^{\circ} \mathrm{C}$ and $10 \%$ nitric acid $\left(\mathrm{HNO}_{3}\right)$ at boiling temperature. After immersing Stellite 6, Stellite 12, Stellite 706, and Stellite 712 in the solutions for 72 hours, the material loss rates were measured as illustrated in Figure 2-3. 


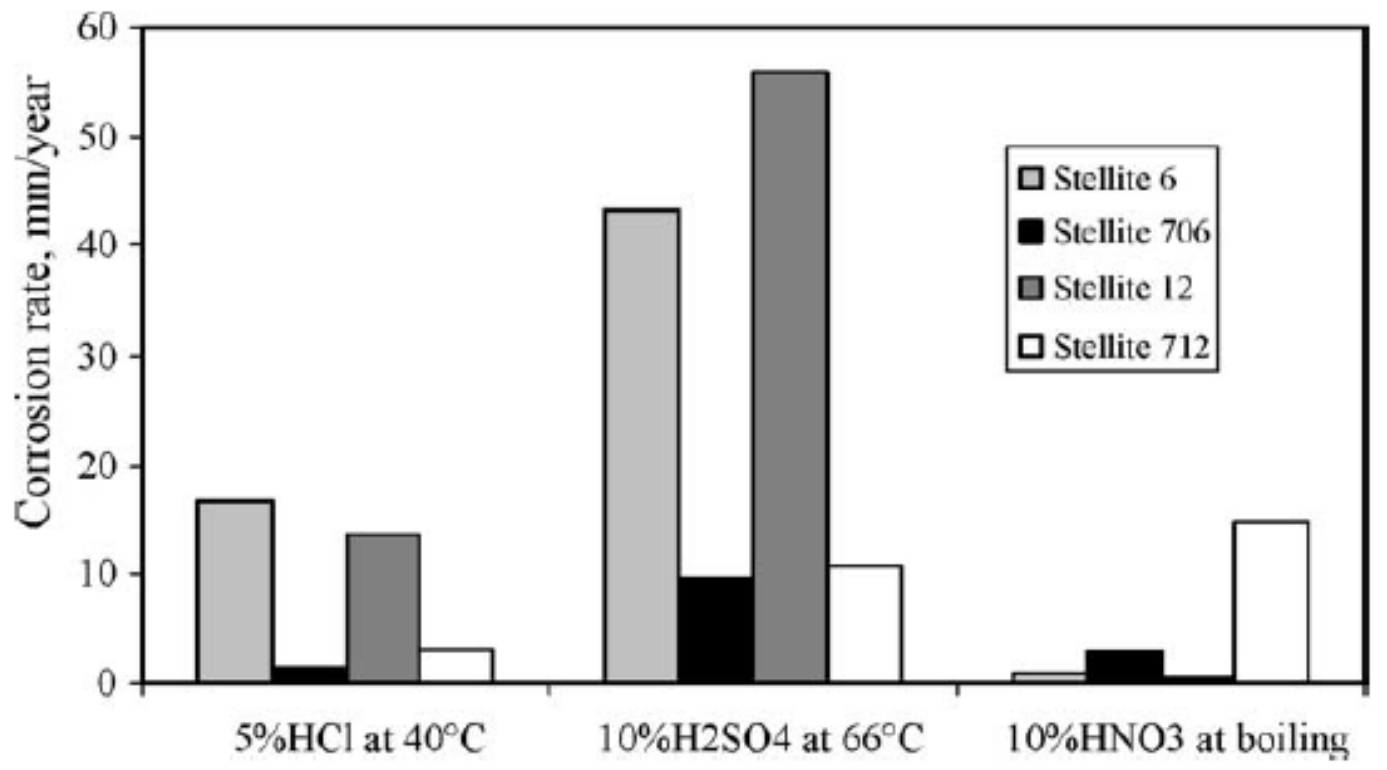

Figure 2-3: Corrosion rates of Stellite 6, Stellite 12, Stellite 706, and Stellite 712 in acid solutions [7]

It can be seen from the results that Stellite 6 and Stellite 12 both containing $\mathrm{W}$ have better performance in an oxidizing environment such as nitric acid, while Stellite 706 and Stellite 712 containing Mo perform better in reducing environments. This is a clear indication that the improved corrosion resistance provided by both $\mathrm{W}$ and Mo are dependent on the type of corrosive environment. Consequently, in boiler feedwater treatment systems, a reducing environment is common $[14,16]$.

\subsubsection{Industrial applications}

In many industries, high-temperature boilers are used as a means for steam or hot water generation. For every boiler used, feedwater treatment is necessary to help remove harmful impurities, avoid boiler failure and downtime, proper chemistry control, prolong service life, reduce fuel cost, and increase boiler efficiency $[14,15,16]$. Table $2-2$ reports some of the common problems that occur with harmful impurities in boiler feedwater and their corresponding treatment methods. 
Table 2-2: Common problems and treatment methods for feedwater $[14,16]$

\begin{tabular}{|c|c|c|}
\hline Issues & Problem & Treatment Method \\
\hline Alkalinity & $\begin{array}{c}\text { Acid attack where } \mathrm{CO}_{2} \text { in } \\
\text { steam is produced eventually } \\
\text { forming carbonic acid }\end{array}$ & $\begin{array}{c}\text { Filming/neutralizing amines } \\
\text { or a combination }\end{array}$ \\
\hline Oxygen & $\begin{array}{c}\text { Oxygen attack at water lines, } \\
\text { boilers, heat exchangers, etc. }\end{array}$ & $\begin{array}{c}\text { Filming amines and } \\
\text { deaeration }\end{array}$ \\
\hline Iron & Iron deposits in boilers & Phosphate, polymers \\
\hline $\mathrm{pH}$ & $\begin{array}{c}\text { Dropping below pH 8.5 } \\
\text { causes corrosion }\end{array}$ & Increased by adding alkalies \\
\hline Silica & Scale build up in boilers & Lime softening \\
\hline Hydrogen Sulfide & Causing corrosion & Chlorination \\
\hline
\end{tabular}

The important information from this table is the addition of neutralizing amines such as cyclohexylamine, morpholine or diethyleminoethanal that can be used to keep dissolved oxygen in water less reactive. These amines can also help keep the water at the alkaline $\mathrm{pH}$ value of 9.5 or above to maintain hydroxide alkalinity $[15,47,16]$. Oxygen attack and acid attack are two of the major problems inside boilers. Dissolved oxygen is extremely aggressive at higher temperatures and causes corrosion on boiler internal surface. The acid attack occurs when feedwater $\mathrm{pH}$ drops below 8.5 where the carbonate in water converts into carbon dioxide, which condenses in water to form carbonic acid $[14,47,16]$. However, the concern here is not the boiler, but the valve trim components in the boiler feedwater treatment system that are in contact with the feedwater during service. Stellite 6 is the most common Stellite alloy used for hardfacing valve trim [1] in valve application and has been the alloy of choice for decades.

Other than hardfacing for valves, Stellite alloys are also used for hardfacing cutting tools such as saws; medical applications, for example, hip implants, dental tools, automotive engine valves; gas turbine combustors, etc. [1]. Examples of different types of Stellite alloys with their general applications in terms of industrial requirements are listed in Table 2-3. 
Table 2-3: Various Stellite alloys and their applications [3]

\begin{tabular}{lc}
\hline \multicolumn{1}{c}{ Alloy } & Application \\
$\begin{array}{l}\text { Cast, P/M, and weld overlay wear resistant } \\
\text { alloys }\end{array}$ & \\
\hline Stellite 1 & Cutter edges, valve seats, bearings \\
\hline Stellite 3 & High-temperature abrasion \\
\hline Stellite 4 & Erosion-corrosion on pumps \\
\hline Stellite 6 & Wear and corrosion resistant with ductility \\
\hline Stellite 12 & High temperature wear resistance \\
\hline Stellite 21 & Ductility and corrosion resistance \\
\hline Stellite 25 & High-temperature nitric acid \\
\hline Stellite 31 & Aerospace engine \\
\hline Stellite 33 & High-temperature wear and abrasion \\
\hline Stellite Star J & High-temperature wear \\
\hline Tribaloy T-400 & Wear and corrosion resistance \\
\hline Tribaloy T-800 & High-temperature abrasion, wear and \\
& corrosion \\
\hline Laves phase wear resistant alloys & \\
\hline Wrought wear resistant alloys & Wear and corrosion resistance \\
\hline Stellite 6B & Wear and corrosion resistance for knives and \\
Stellite 6K & scrapers \\
\hline
\end{tabular}

\subsection{Amine Environment}

There are essentially two types of amines, film-forming amines and neutralizing amines [14]. Film forming amines form a continuous layer on the metal surface to protect it from outside aqueous corrosive attacks. The corrosive attacks can be as a form of oxygen attack and/or acid attack in applications. Two common types of filming amines are ethoxylated soya amine (ESA) and octadecylamine (ODA) [14]. However, filming amines are insoluble in water so that it cannot be used as a testing electrolyte solution for corrosion experiments [17]. Neutralizing amines, on the other hand, are high $\mathrm{pH}$ (high basicity) amines. It can be added directly in boiler feedwater to neutralize the formation of carbonic acid and to also keep the feedwater alkaline. There are three most common types of neutralizing amines such as cyclohexylamine, diethylaminoethanal (DEAE) and morpholine [14]. 
Morpholine is chosen as the corrosive medium in the present corrosion study of Stellite alloys. Morpholine is one of the three common additives used in power plant steam systems because its volatility is similar to water and it is non-volatile at room temperature [17]. Morpholine readily mixes with water to form a solution. Morpholine also decomposes slowly in the absence of oxygen at high temperature and pressure, which is useful in steam/boiler systems [17].

Morpholine is a basic organic compound having the features of both ether and amine functional group in its 2D chemical structure, as shown in Figure 2-4. The chemical formula of morpholine is $\mathrm{C}_{4} \mathrm{H}_{9} \mathrm{NO}$. Its vapor pressure at room temperature is much less than air at $10 \mathrm{mmHg}$ and its vapor density is higher relative to air with a value of 3 (air $=1$ ) [17]. With its volatility like water and its density higher than air, morpholine hardly evaporates at room temperature, which would allow the $\mathrm{pH}$ of the solution to remain approximately constant for many hours when it is mixed into water.

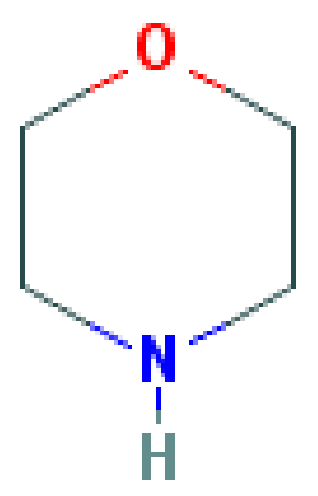

Figure 2-4: 2D structure of morpholine [17]

\subsection{Previous Research}

Previous research done on different types of Stellite alloys investigate general and localized corrosion behaviour as well as oxide integrity primarily in salt and acidic mediums. Rosalbino and 
Scavino investigated the corrosion behaviour of as-cast Stellite 6 and hot isostatic pressing (HIP) processed Stellite 6 in 3.5 wt.\% $\mathrm{NaCl}$ solution using EIS and potentiodynamic polarization methods [18]. The experimental results showed that the HIPed Stellite 6 had much better corrosion performance compared to as-cast Stellite 6. This is because the carbides in HIPed Stellite 6 are uniformly distributed around the Co solid solution matrix in spheroidal form rather than the dendritic carbides in as-cast Stellite 6, as shown in Figure 2-5. Normally, Stellite alloys do not require heat treatment after casting, however, this research shows HIPed process is one additional method that can enhance the performance of Stellite 6 that has low Mo content.

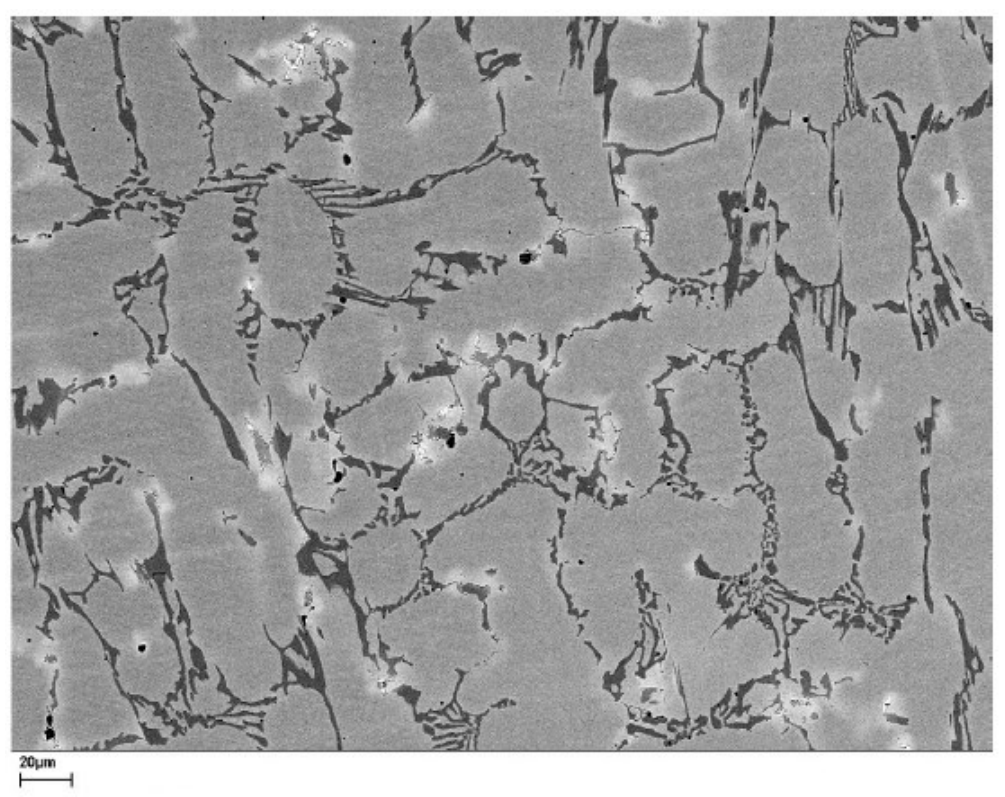

(a) 


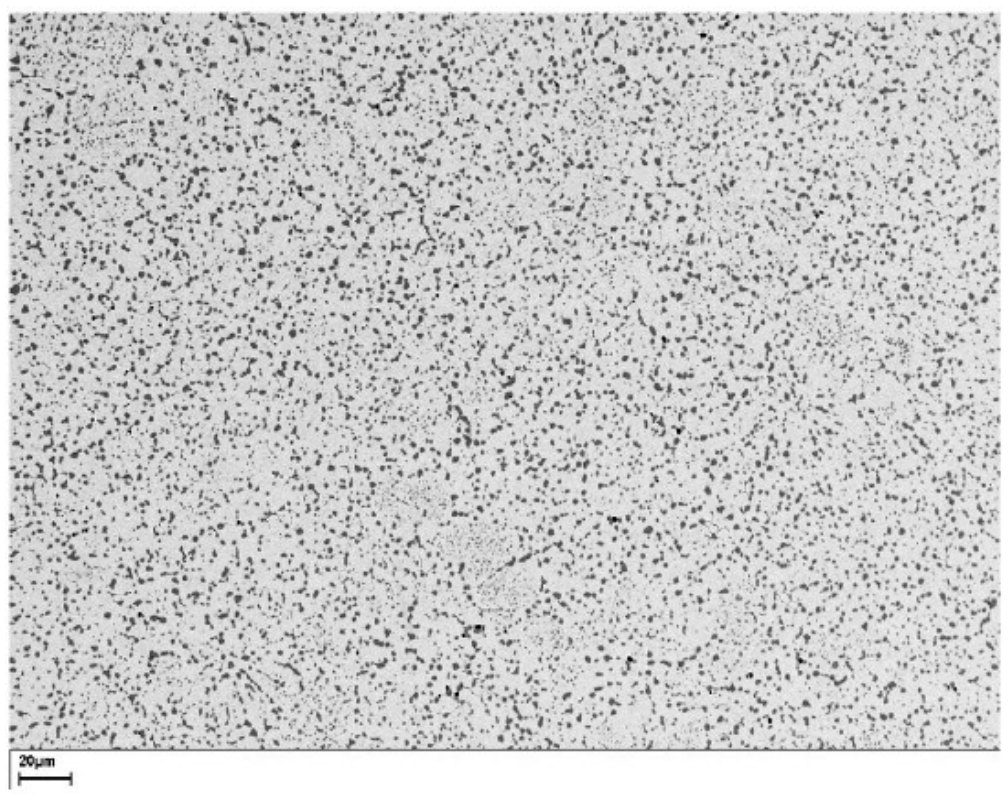

(b)

Figure 2-5: SEM microstructure of Stellite 6 (a) as-cast and (b) HIPed [18]

Their research also showed the importance of open circuit potential (OCP) and how it can be used as an indicator of an alloy's peak corrosion performance. Figure 2-6 compares the difference in OCP after 168 hours of exposure to $3.5 \%$ wt. $\% \mathrm{NaCl}$ solution between as-cast and HIPed Stellite 6. Figure 2-6 shows that HIPed Stellite 6 has a lower OCP stability value after 168 hours of exposure and as a result, the polarization forward curve in Figure 2-7 shows a longer sustaining spontaneous passive region at higher potentials for the HIPed Stellite 6 before transitioning into transpassive behaviour. 


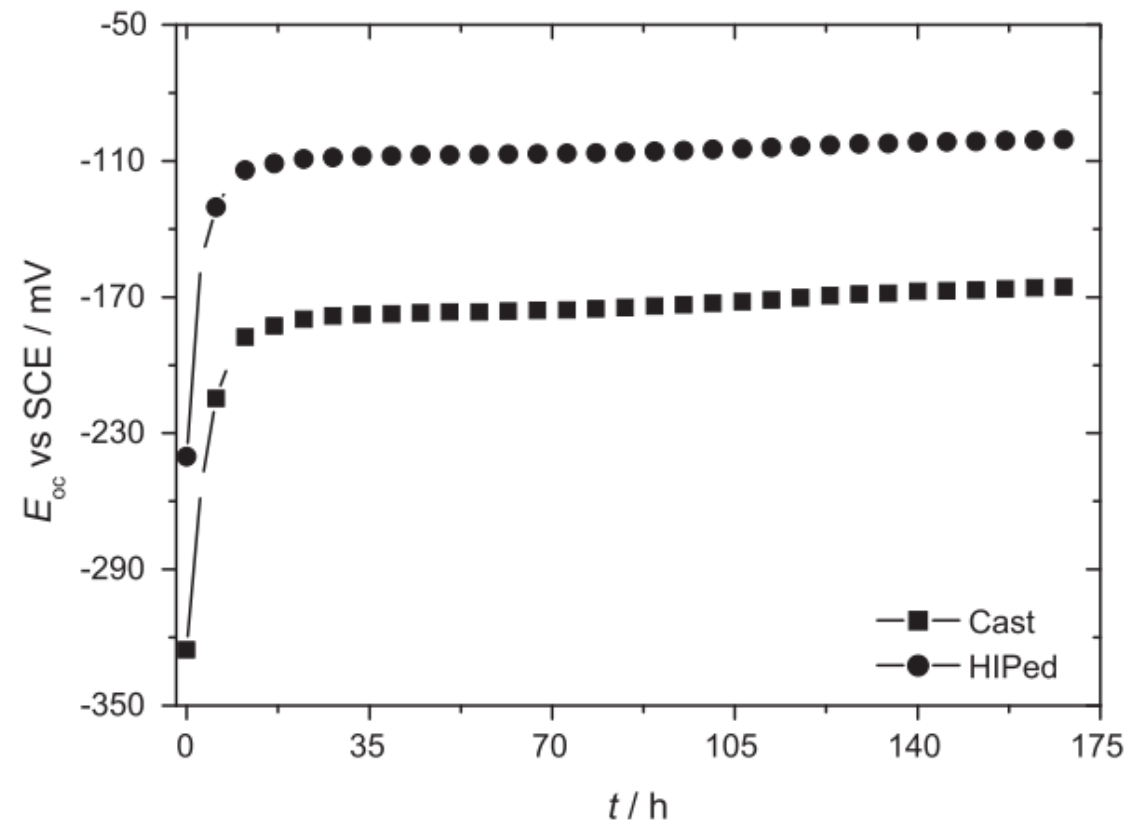

Figure 2-6: OCP curves for as-cast and HIPed Stellite 6 after $168 \mathrm{hr}$ exposure to 3.5 wt.\% NaCl solution [18]

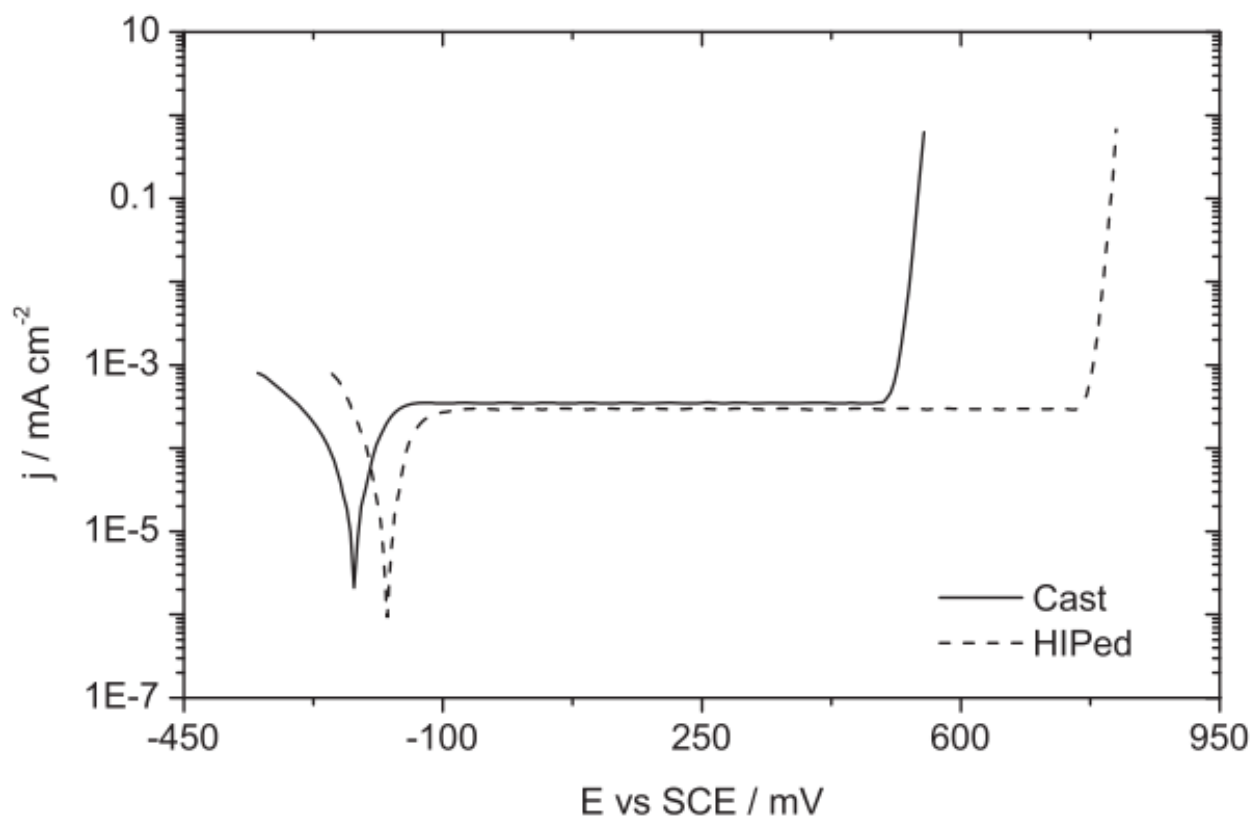

Figure 2-7: Potentiodynamic polarization curves of as-cast and HIPed Stellite 6 after $168 \mathrm{hr}$ exposure to 3.5 wt.\% NaCl solution [18] 
The different times of exposure also showed a difference in the EIS results between the two alloys from different processes. Higher $R_{c t}$ values of the HIPed Stellite 6 are compared to that of the ascast Stellite 6, as shown in Table 2-4 for longer exposure times, which are an indication that prolonged exposure to the corrosive solution had improved the protective property of the oxide layer displaying higher stability [18].

Table 2-4: EIS data for as-cast and HIPed Stellite 6 in 3.5 wt.\% $\mathrm{NaCl}$ solution [18]

\begin{tabular}{|c|c|c|c|c|}
\hline Sample & $\mathrm{R} \Omega\left(\Omega \mathrm{cm}^{2}\right)$ & $R_{\mathrm{ct}}\left(\mathrm{k} \Omega \mathrm{cm}^{2}\right)$ & $10^{6} \times Q_{\mathrm{dl}}\left(\Omega^{-1} \mathrm{~cm}^{-2} \mathrm{~s}^{\mathrm{n}}\right)$ & $\mathbf{n}$ \\
\hline \multicolumn{5}{|l|}{ Cast } \\
\hline 1 & 1.32 & 200.7 & 87.1 & 0.90 \\
\hline 24 & 1.45 & 380.2 & 71.8 & 0.91 \\
\hline 48 & 1.54 & 539.8 & 58.9 & 0.92 \\
\hline 72 & 1.65 & 659.3 & 45.6 & 0.93 \\
\hline 96 & 1.74 & 700.1 & 35.4 & 0.94 \\
\hline 120 & 1.82 & 721.1 & 27.9 & 0.95 \\
\hline 144 & 1.89 & 743.6 & 22.1 & 0.96 \\
\hline 168 & 1.97 & 751.9 & 17.6 & 0.97 \\
\hline \multicolumn{5}{|l|}{ HIPed } \\
\hline 1 & 1.43 & 900.3 & 65.4 & 0.92 \\
\hline 24 & 1.51 & 1050.4 & 54.1 & 0.93 \\
\hline 48 & 1.60 & 1170.2 & 43.2 & 0.94 \\
\hline 72 & 1.71 & 1300.6 & 35.5 & 0.95 \\
\hline 96 & 1.82 & 1400.1 & 26.3 & 0.96 \\
\hline 120 & 1.90 & 1450.5 & 17.9 & 0.97 \\
\hline 144 & 1.97 & 1500.7 & 13.7 & 0.98 \\
\hline 168 & 2.05 & 1525.2 & 10.1 & 0.99 \\
\hline
\end{tabular}

Yi et al. investigated Stellite 6 and $17-4 \mathrm{PH}(17.5 \% \mathrm{Cr}, 5 \% \mathrm{Ni}, 3.5 \% \mathrm{Cu}$ in weight) stainless steel corrosion performance in morpholine, cyclohexylamine and sodium hydroxide solutions. The solutions were each at $9.5 \mathrm{pH}$ following the industrial standard of feedwater $\mathrm{pH}$ level [5]. Table 2-5 gives the summarized results of the polarization test for both alloys at $25^{\circ} \mathrm{C}$ after 24 hours OCP. It can be inferred from the values that Stellite 6 generally performed better in resistance to oxidation because its general corrosion performance was better $\left(I_{c o r r}\right.$ and $\left.R_{p}\right)$. 
Table 2-5: Polarization parameters of Stellite 6 and 17-4PH stainless steel in basic environments [5]

\begin{tabular}{|c|c|c|c|}
\hline Alloy & $E_{\text {corr }}(\mathbf{m V})$ & Icorr $\left(\mathrm{nA} / \mathrm{cm}^{2}\right)$ & $R_{p}\left(\mathrm{k} \Omega \cdot \mathrm{cm}^{2}\right)$ \\
\hline \multicolumn{4}{|l|}{ Morpholine } \\
\hline Stellite 6 & -90 & 20.8 & 2700 \\
\hline 17-4PH SS & -170 & 22.0 & 1500 \\
\hline \multicolumn{4}{|c|}{ Cyclohexylamine } \\
\hline Stellite 6 & -160 & 7.5 & 4800 \\
\hline 17-4PH SS & -180 & 25.0 & 1300 \\
\hline \multicolumn{4}{|l|}{$\mathrm{NaOH}$} \\
\hline Stellite 6 & -230 & 10.1 & 3400 \\
\hline 17-4PH SS & -190 & 25.0 & 1300 \\
\hline
\end{tabular}

Furthermore, looking at the hysteresis loop, as shown in Figure 2-8, Stellite 6 had better localized corrosion performance at higher potentials displaying a stable hysteresis, whereas $17-4 \mathrm{PH}$ steel showed negative hysteresis. This behaviour was observed to be similar for both alloys in all three basic solutions tested. Negative hysteresis generally refers to the alloy having a higher current density in its reverse sweep compared to forward sweep at the same potential. This research shows that Stellite 6 has better general and localized corrosion performance compared to 17-4PH stainless steel primarily due to the difference in $\mathrm{Cr}$ content. Stellite 6 having nearly double $\mathrm{Cr}$ content would indicate its corrosion in addition to wear performance will be generally better. 


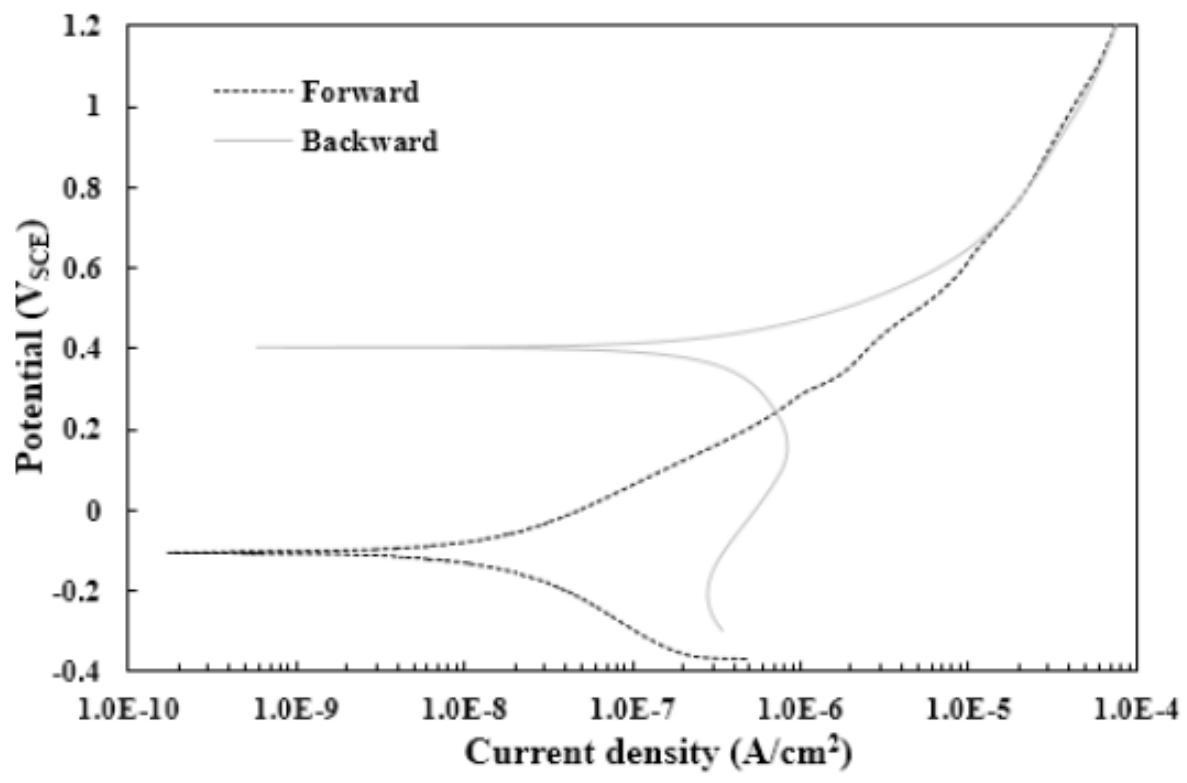

(a)

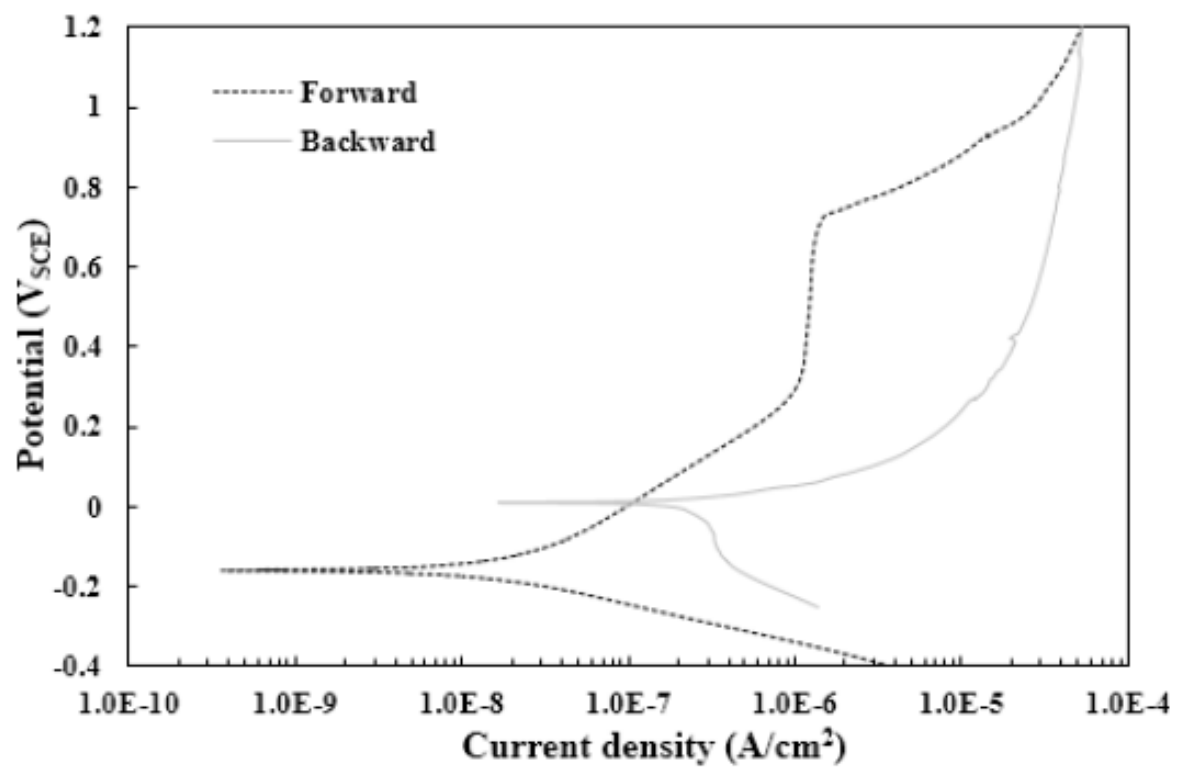

(b)

Figure 2-8: Cyclic polarization curve of (a) Stellite 6 and (b) 17-4PH stainless steel in morpholine solution at pH 9.5 [5] 
Liu et al. investigated the erosion-corrosion performance of two new low-C high-Mo Stellite alloys, Stellite 22 and Stellite 728, compared with Stellite 21 and Stellite 6 [11]. Their compositions are given in Table 2-6.

Table 2-6: Composition table of Stellite alloys [11]

\begin{tabular}{|c|c|c|c|c|c|c|c|c|c|}
\hline Alloy & Cr & W & Mo & C & Fe & Ni & Si & Mn & Nb \\
\hline Stellite 22 & 27 & & 11 & 0.25 & 3 & 2.75 & 1 & 1 & \\
\hline Stellite 728 & 24.2 & & 11.8 & 0.35 & 1 & 3.8 & 0.45 & 0.52 & 2.07 \\
\hline Stellite 21 & 27 & & 5.5 & 0.25 & 3 & 2.75 & 1 & 1 & \\
\hline Stellite 6 & 28.5 & 4.5 & 1.5 & 1.2 & 3 & 3 & 1.5 & 1 & \\
\hline
\end{tabular}

The general corrosion data given in Table 2-7 indicates that Stellite 22 and Stellite 21 displayed better performance, however, the influence of having different Mo composition is not seen due to low OCP duration at approximately two minutes. Nevertheless, with low OCP the Stellite alloys with higher Mo displayed better performance in comparison to Stellite 6. Stellite 6, however, did display better erosion performance in comparison due to its higher $\mathrm{C}$ content is shown in Figure 2-9. The erosion test, in this case, was a solid particle erosion test at two different impingement angles and the results favor Stellite 6 due to its higher volume fraction of $\mathrm{Cr}$ and $\mathrm{W}$ carbides. This research is somewhat analogous to the current research showing the effect Mo has on general corrosion properties.

Table 2-7: Polarization parameters of Stellite alloys in 3.5 wt.\% NaCl solution [11]

\begin{tabular}{|c|c|c|c|}
\hline Alloy & $E_{\text {corr }}(\mathrm{mV})$ & $I_{\text {corr }}\left(\mathrm{nA} / \mathbf{c m}^{2}\right)$ & $R_{p}\left(\mathrm{k} \Omega \cdot \mathrm{cm}^{2}\right)$ \\
\hline Stellite 22 & -325 & 40 & 342 \\
\hline Stellite 728 & -215 & 90 & 141 \\
\hline Stellite 21 & -210 & 40 & 340 \\
\hline Stellite 6 & -320 & 140 & 90 \\
\hline
\end{tabular}




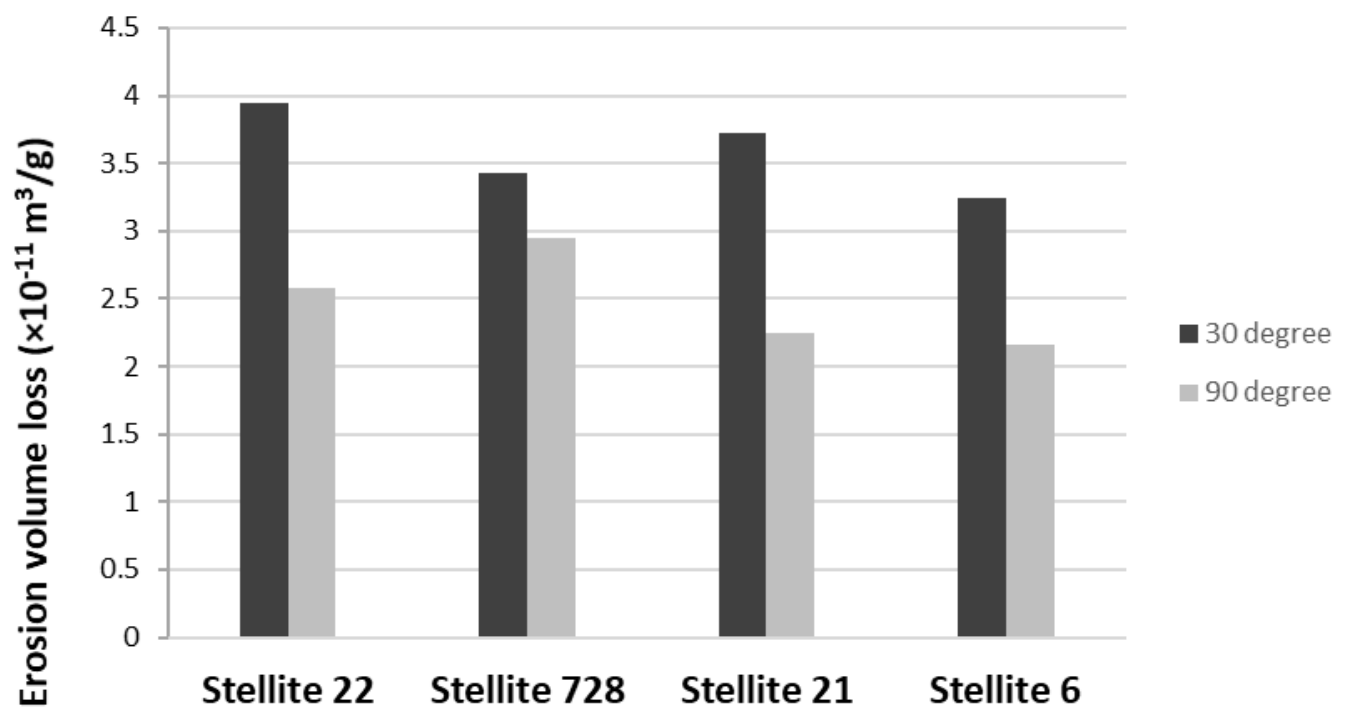

Figure 2-9: Erosion volume loss comparison of Stellite alloys [11]

Zhang et al. investigated the corrosion and carbide size influence on pitting of Stellite 6B and Stellite 6K alloys, which are wrought Stellite alloys, using 3.5 wt.\% $\mathrm{NaCl}$ and Green Death Solution [10]. The composition of the alloys is given in Table 2-8.

Table 2-8: Composition of Stellite 6B and Stellite 6K [10]

\begin{tabular}{|c|c|c|c|c|c|c|c|c|}
\hline Alloy & Cr & W & Mo & C & Fe & Ni & Si & Mn \\
\hline Stellite 6B & 30 & 4 & 1.5 & 1 & 3 & 2.5 & 0.7 & 1.4 \\
\hline Stellite 6K & 30 & 4.5 & 1.5 & 1.6 & 3 & 3 & 2 & 2 \\
\hline
\end{tabular}

The polarization plots are presented in Figure 2-10 showing that wrought Stellite 6B and Stellite $6 \mathrm{~K}$ exhibited similar general corrosion behaviour and spontaneous passive film formation. However, Stellite $6 \mathrm{~K}$, which contains higher $\mathrm{C}$ content, had a greater current density at the oxidation potential. With a slight increase in $\mathrm{C}$ content, the results show that it can be a detriment to corrosion performance. The EIS spectra of the alloys tested in $3.5 \mathrm{wt} . \% \mathrm{NaCl}$ solution are presented in Figure 2-11 and Figure 2-12, indicating that the oxide layer integrity of both the Stellite alloys does not significantly change due to the same amount of $\mathrm{Cr}$ and Mo content. 


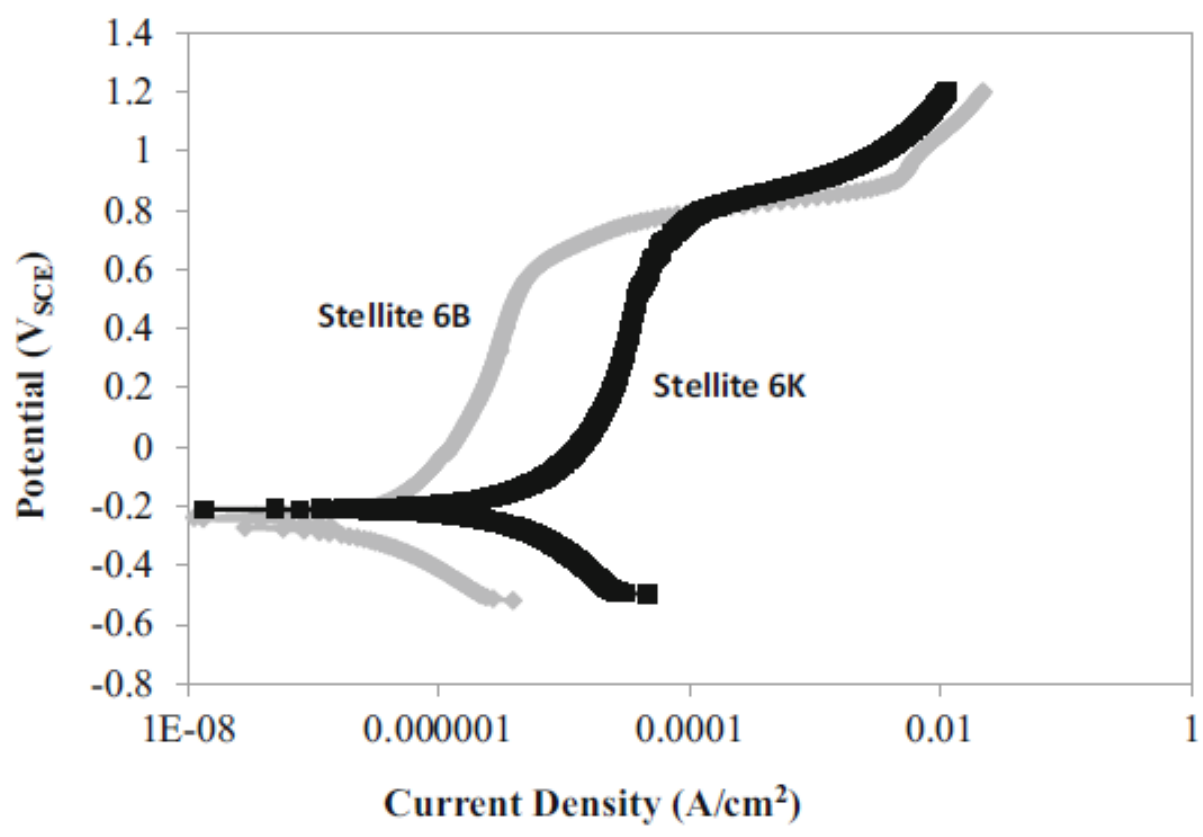

(a)

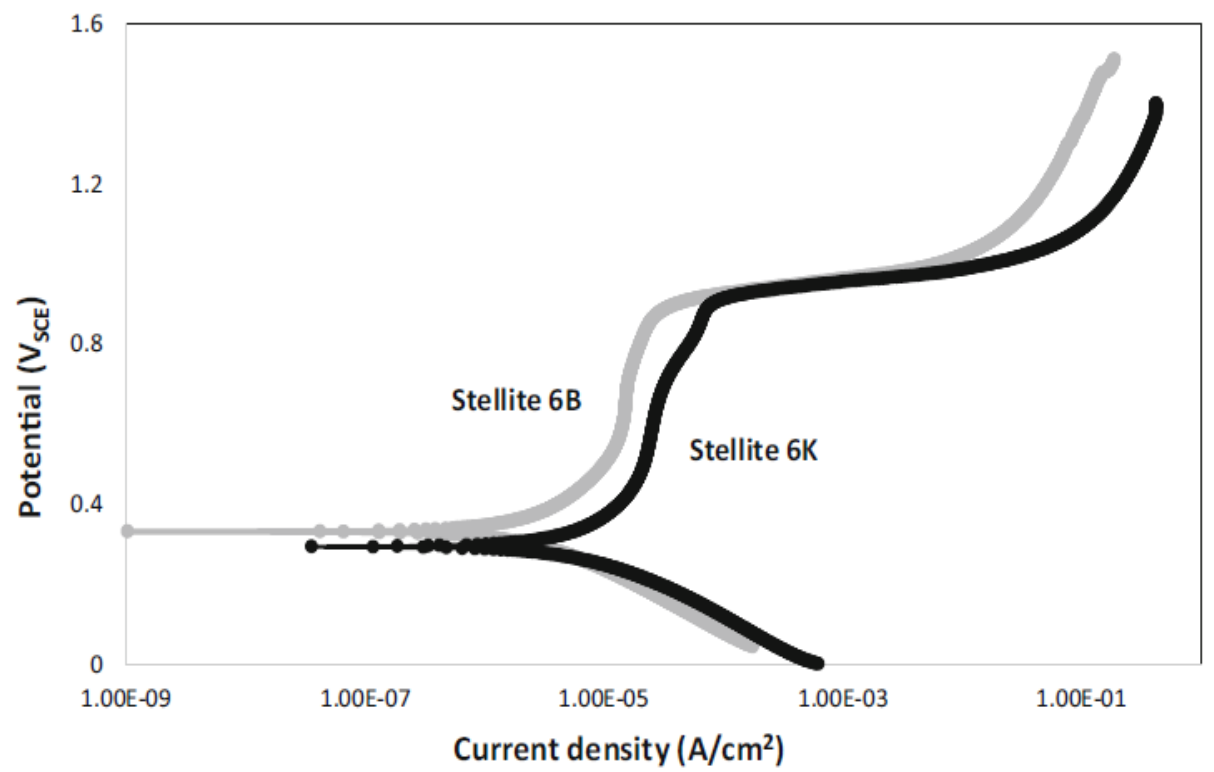

(b)

Figure 2-10: Polarization curves for Stellite 6B and Stellite $6 \mathrm{~K}$ in (a) $3.5 \mathrm{wt} . \% \mathrm{NaCl}$ solution and (b) Green Death solution [10] 


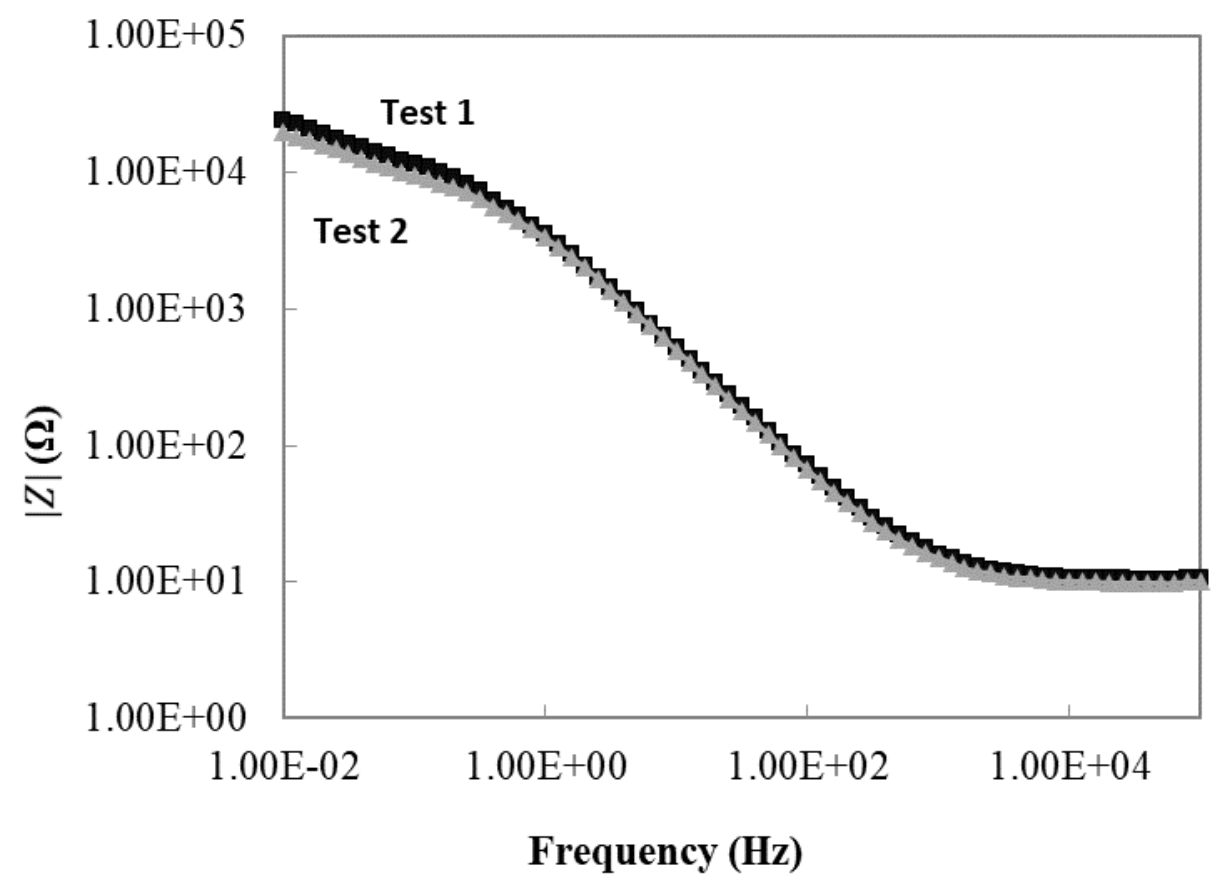

(a)

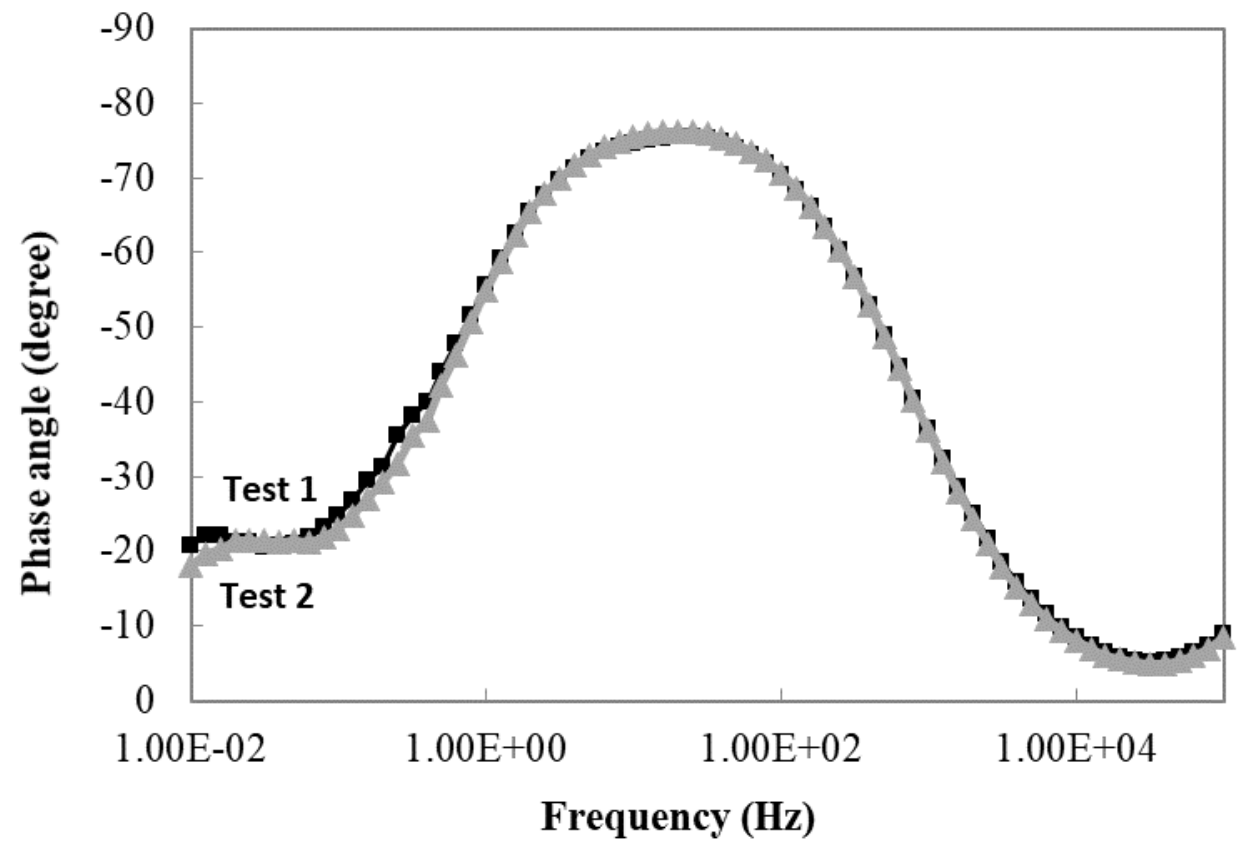

(b)

Figure 2-11: Bode plot of Stellite 6B tested in $3.5 \mathrm{wt} \%$ NaCl solution: (a) impedance and (b) phase angle [6] 


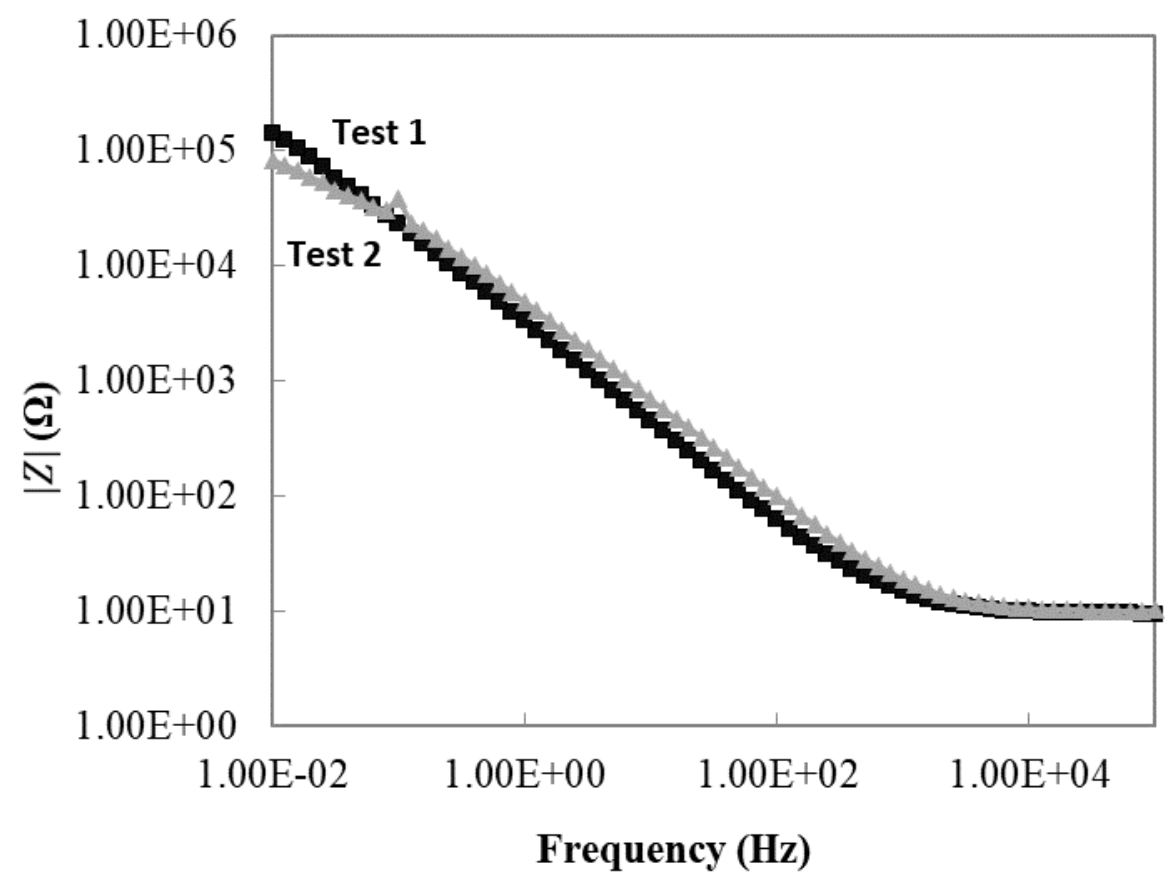

(a)

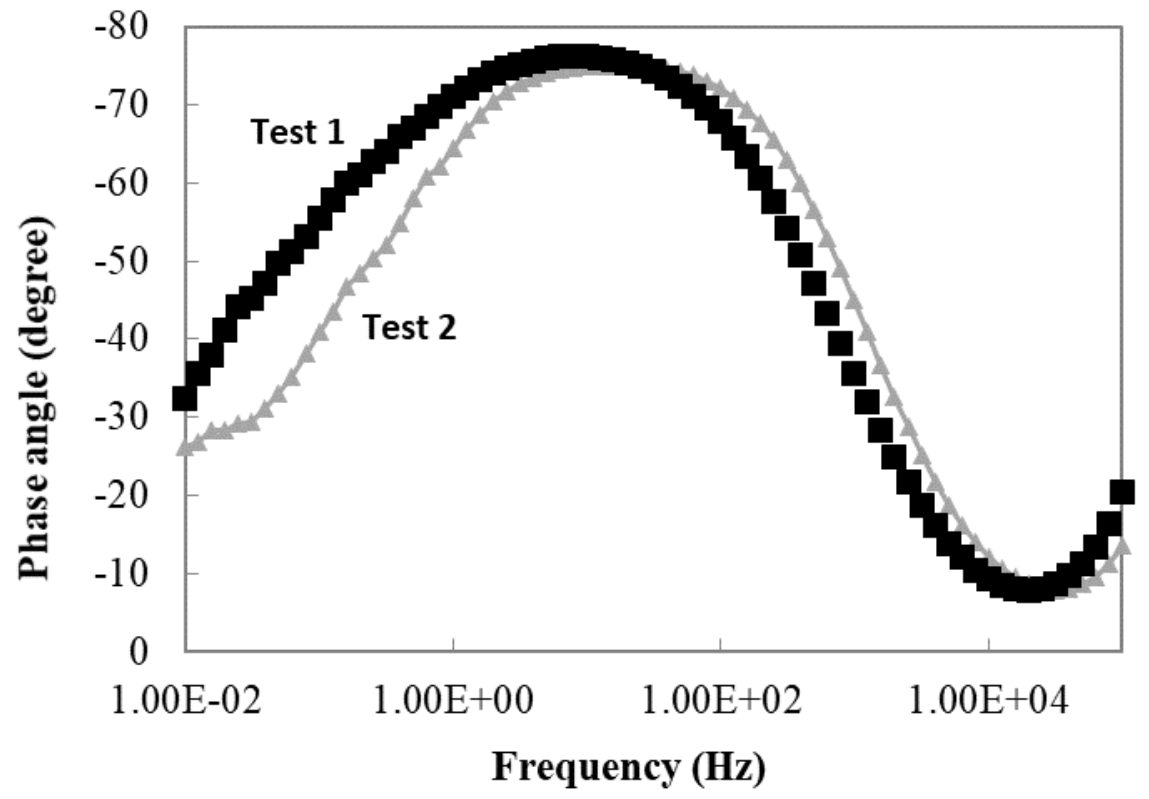

(b)

Figure 2-12: Bode plot of Stellite 6K tested in 3.5 wt.\% NaCl solution: (a) impedance and (b) phase angle [6] 
$\mathrm{Hu}$ et al. investigated the corrosion resistance of Stellite 720 and Stellite 21 in simulated human body fluid (Hanks solution with $\mathrm{pH} 7.4$ at $37^{\circ} \mathrm{C}$ ) using electrochemical and immersion corrosion tests. The composition is given in Table 2-9 indicating a clear difference in Mo content.

Table 2-9: Composition of Stellite 21 and Stellite 720 [20]

\begin{tabular}{|c|c|c|c|c|c|c|c|}
\hline Alloy & Cr & Mo & C & Fe & Ni & Si & Mn \\
\hline Stellite 21 & 27 & 5.5 & 0.25 & 3 & 2.75 & 1 & 1 \\
\hline Stellite 720 & 33 & 18 & 2.5 & 3 & 3 & 1.5 & 1.5 \\
\hline
\end{tabular}

The EIS spectra for the alloys are shown in Figure 2-13 and the EIS parameter fitting results revealed that Stellite 720 had the better oxide layer integrity. The oxide film in Stellite 21 was more susceptible to anodic perturbations that might occur during routine application in vivo. The oxide film on Stellite 720 surface appeared to re-passivate more quickly to a more protective oxide [20]. This research suggests that having additional Mo despite the increase in $\mathrm{C}$ content compensates the effect of having more interfaces.

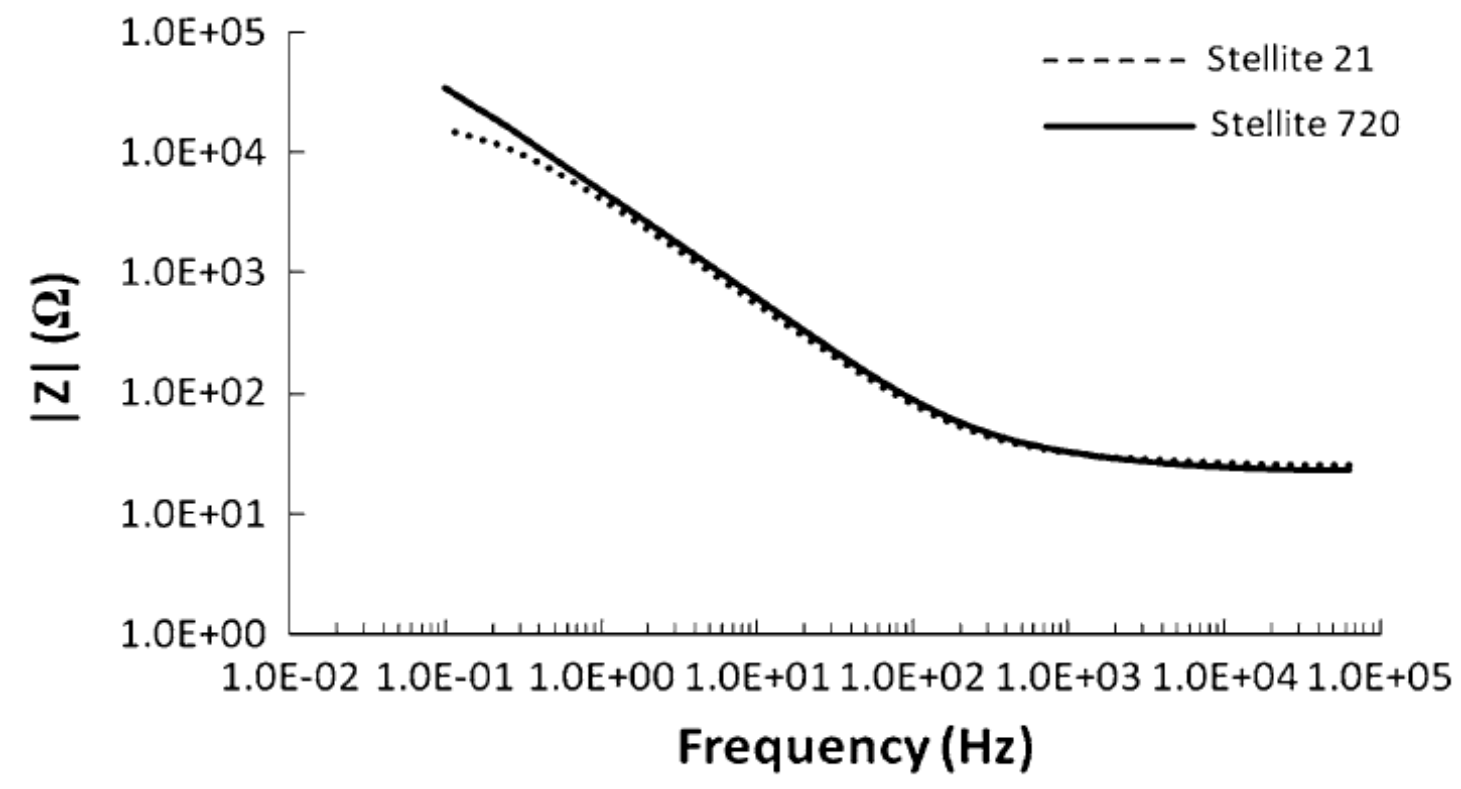

(a) 


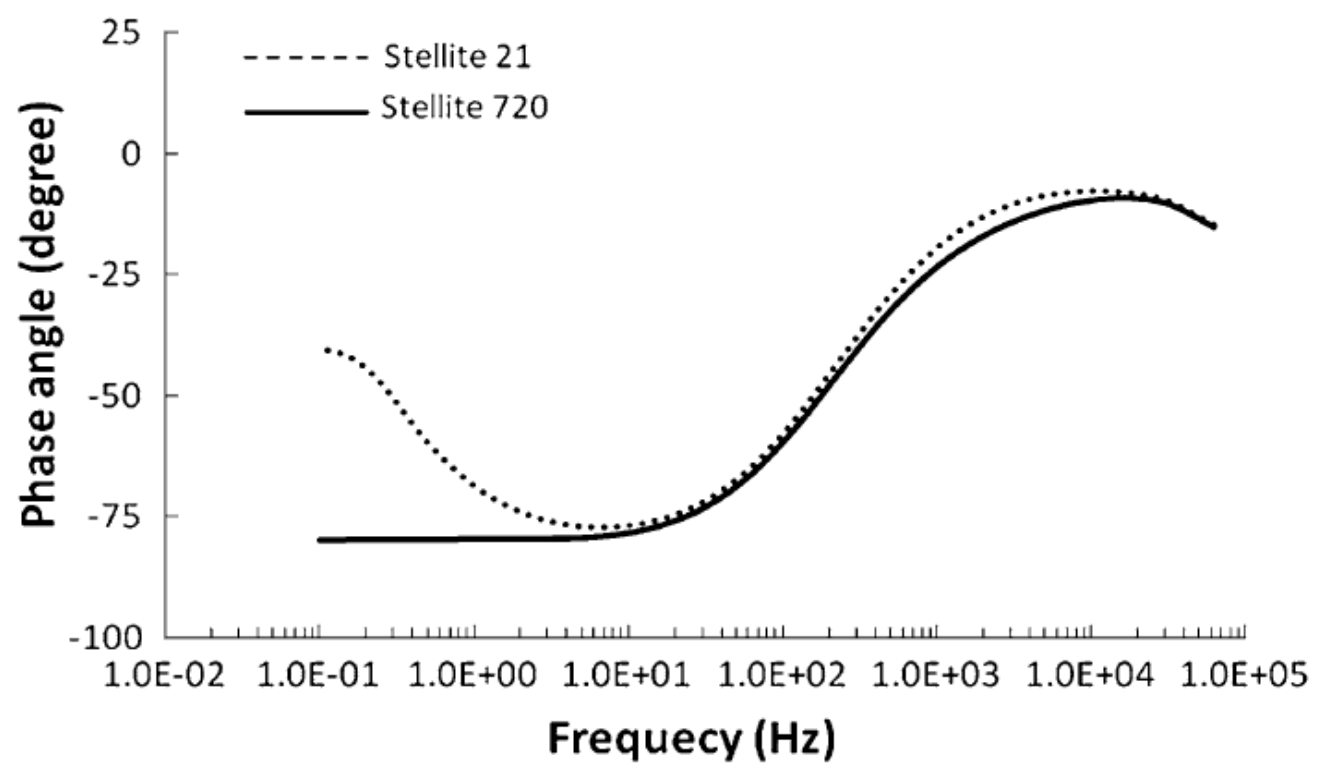

(b)

Figure 2-13: Bode plot of Stellite alloys in Hanks solution: (a) impedance and (b) phase angle [20]

The effect of Mo on corrosion was studied by Olefjord et al. by examining the surface composition of stainless steel during anodic dissolution and passivation in $0.1 \mathrm{M} \mathrm{HCl}+0.4 \mathrm{M} \mathrm{NaCl}$ using $\mathrm{X}$ ray photoelectron spectroscopy (XPS) shown in Figure 2-14. Based on the XPS data and its correlation with polarization tests, the conclusion was reached that Mo has a strong influence on the corrosion properties of stainless steels because the $\mathrm{Mo}^{4+}$ and hexavalent $\left(\mathrm{Mo}^{6+}\right)$ oxides forming on the surface of a metal can block active sites [35]. Similar research from Sugimoto et al. and Hashimoto et al. in relation to Mo influence also reported that the Cr oxide film was heterogeneous, thus microcracks were a source where currents can leak through [36, 37]. In the corrosive solution, $\mathrm{Mo}^{6+}$ ions were formed in the cracked or porous regions of the oxide film, thus reducing the size of the active sites and effectively slowing down the dissolution [35]. 


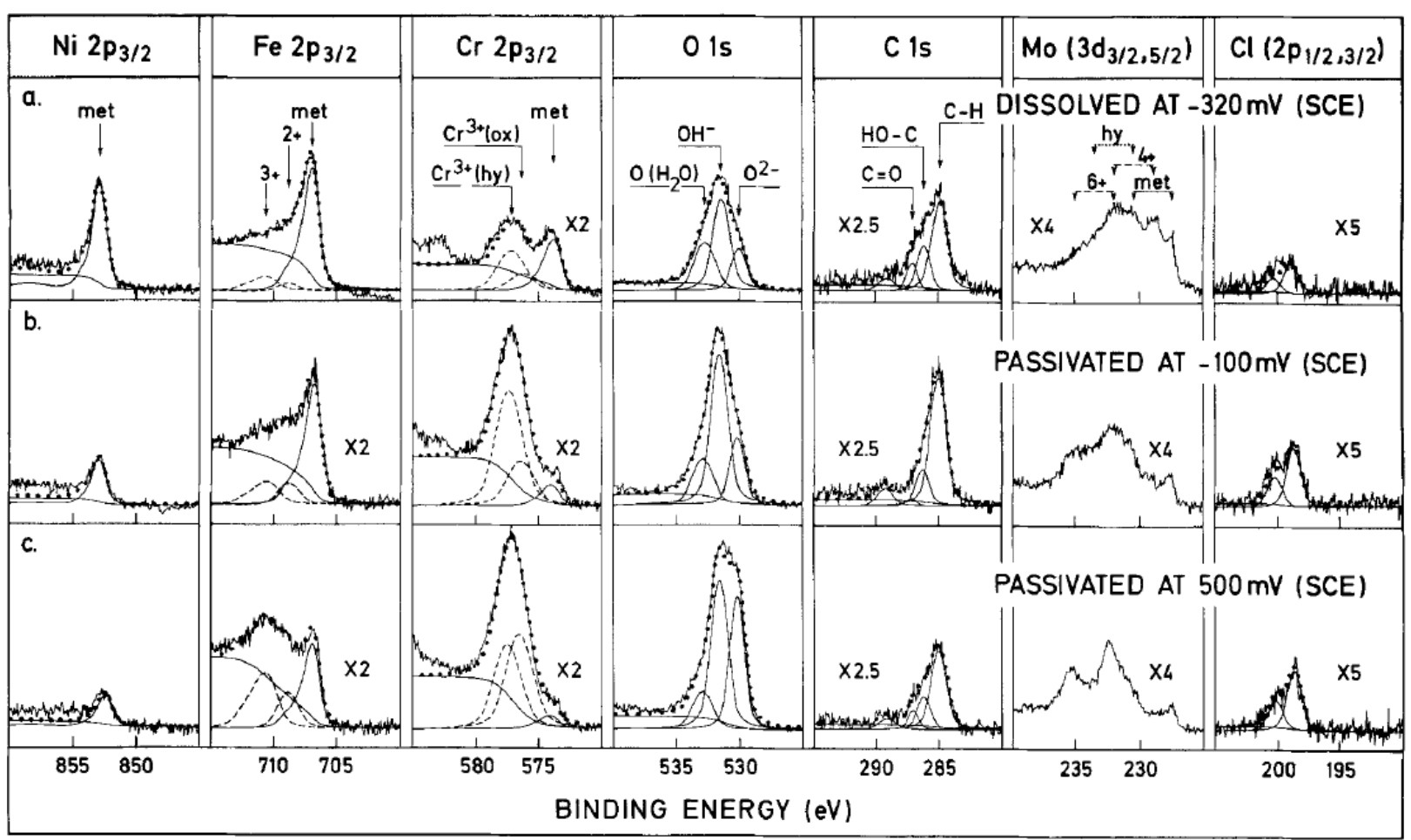

Figure 2-14: XPS spectra of Fe-18Cr-14.3Ni-2.5Mo alloy [35]

Bastidas et al. researched Mo influence on AISI 316L stainless steel in 5 wt. $\% \mathrm{NaCl}$ compared with AISI 304L steel without Mo. By using XPS analysis shown in Figure 2-15, it was found that Mo had the capability to form complex chlorides such as insoluble salt films containing $\mathrm{Mo}^{4+}$ ions [40], which lowered the concentration of chloride ions within pits hence allowing repassivation, leading to better oxide stability during recovery [40]. Mo has a strong interaction with $\mathrm{Cr}$ when forming passive films in an aggressive chloride environment and its oxide state depends on the potential as well as the $\mathrm{pH}$ level of the aqueous solution [35-37]. 


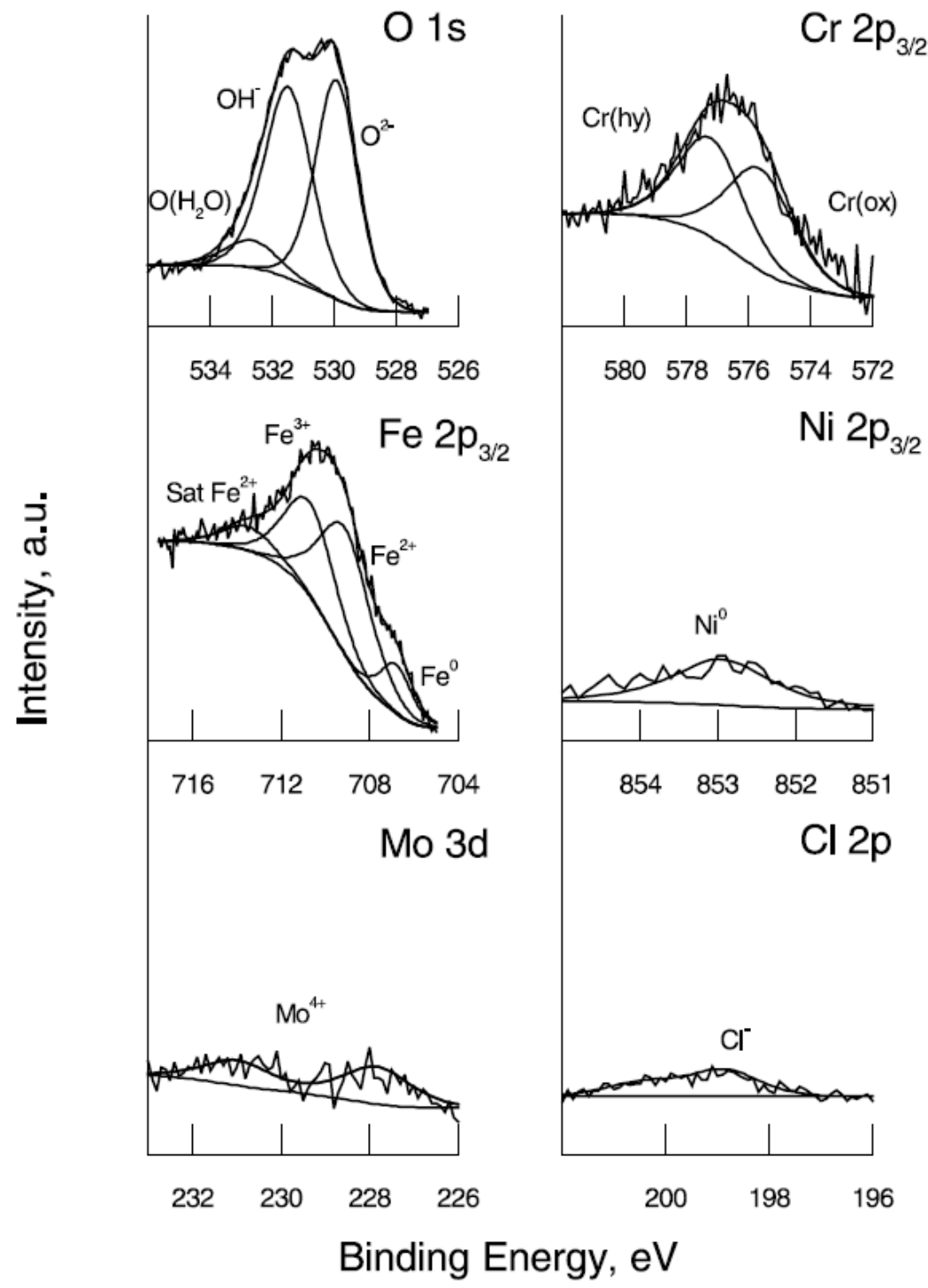

Figure 2-15: XPS spectra of AISI 316L [40]

\subsection{Methods of Corrosion Study}

\subsubsection{Electrochemical impedance spectroscopy (EIS)}

EIS is a non-destructive corrosion test method of materials to provide qualitative and quantitative information about changes in an electrochemical system. Essentially it is a steady-state technique 
that can provide information about a specimen's oxide layer integrity over time. EIS is done by applying a small sinusoidal alternating voltage on a specimen surface over a wide range of frequencies, thus an electrical response of the specimen is measured $[25,26]$. When working with EIS, a three-electrode cell is used where components such as a working electrode (WE), a counter electrode (CE) and a reference electrode (RE) are needed. The WE is applied to the specimen under investigation, $\mathrm{CE}$ is the main current source and $\mathrm{RE}$ is a reference voltage [25]. The concept of EIS is derived from Ohm's law where it defines simple electrical resistance that depends on voltage over current, as expressed by Eq (1). However, this concept is limited to the resistor circuit element [25]. More components exist such as inductor, capacitor and constant phase element, which exhibit more complex behaviour and the Ohm's law can be modified as shown in Eq (2) by replacing the resistor element with impedance, $Z$, which is a circuit's tendency to impede the flow of current. The adjusted mathematical model is expressed as [26]:

$$
\begin{aligned}
& R=\frac{V}{I} \\
& Z=\frac{V_{\omega}}{I_{\omega}}
\end{aligned}
$$

where $V_{\omega}$ is frequency-dependent potential and $I_{\omega}$ is frequency-dependent current. The excitation and response in EIS are shown in Figure 2-16, where the difference between applied voltage and measured current is illustrated along with their phase shift. The phase shift serves as an indication of whether a specimen displays capacitive or inductive behaviour. 


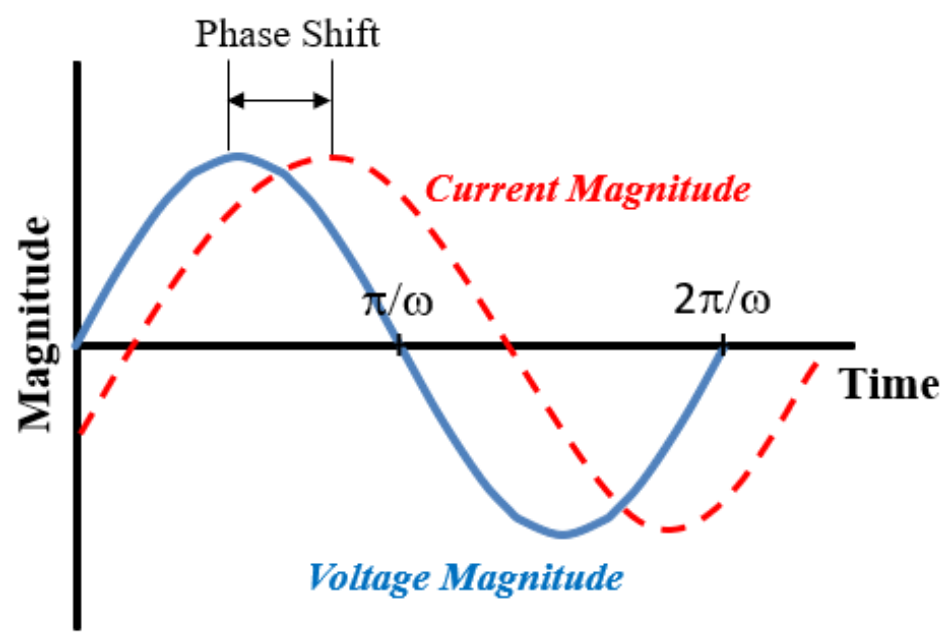

Figure 2-16: An example of a phase shift between the applied voltage and current response during the EIS process [24]

Figure 2-16 shows a sine wave voltage applied to an electrochemical system and its current magnitude response. The sine wave represents $\mathrm{AC}$ voltage and the current response is also an $\mathrm{AC}$ current, but both the curves are shifted in time due to charge and energy storage in the electrochemical system [25]. For example, if one cycle $\left(360^{\circ}\right)$ in a sine wave takes 1 second to complete and the time shift between the voltage and current sine wave is 0.1 second, then the phase shift is $36^{\circ}[25]$. The time shift can be represented as phase angle $\Phi$, and as seen from Figure 2-16, the voltage is leading the current, which means its peak amplitude occurs before the peak amplitude of the current. For this case the phase angle is positive and if the current is to lead voltage, then the phase angle is negative $[25,26]$.

The impedance $Z$ is characterized by three parameters, the impedance magnitude $|Z|$, the phase angle $\Phi$, and frequency $f$. These three parameters are used to plot what is known as the Bode plot, as shown in Figure 2-17(a). The Bode plot is characterized by two separate curves showing the impedance and phase angle. The impedance curve of the plot displays the oxide layer behaviour 
like that of a capacitor, inductor and resistor, which is correlated with the phase angle curve of the plot showing the phase shift. The frequency range can start over $10^{6} \mathrm{~Hz}$ down to $10^{-3} \mathrm{~Hz}$. The Nyquist plot is a way to indicate the presence of resistive or passive films on the alloy surface, as shown in Figure 2-17(b) where $R_{u}$ in the plot represents the solution resistance and $R_{p}$ represents the parallel resistance [26]. For this research, the bode plot is utilized primarily to interpret the surface behaviour of the Stellite alloys. The Nyquist plot is used to determine both the solution and parallel resistance values.

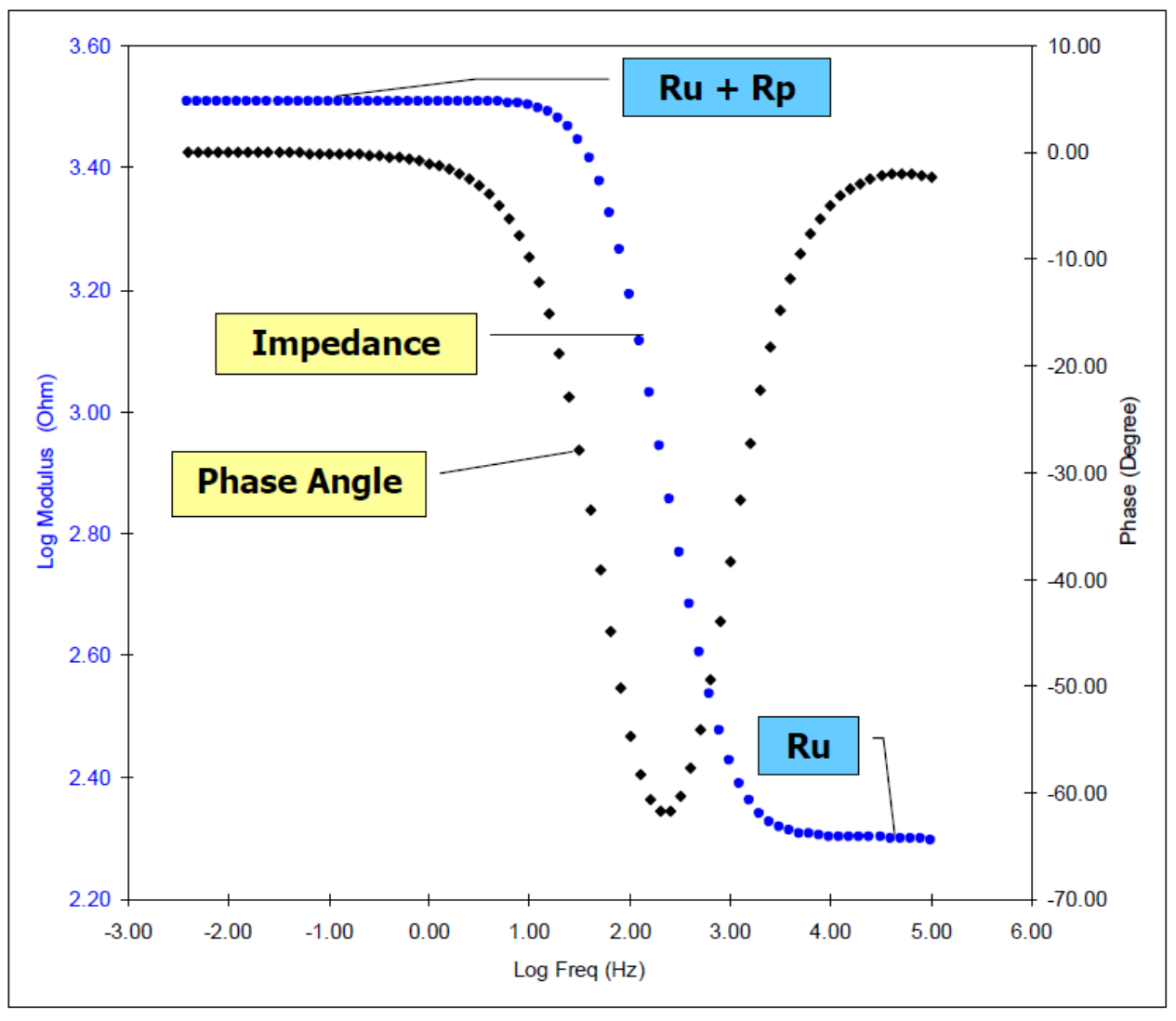

(a) 


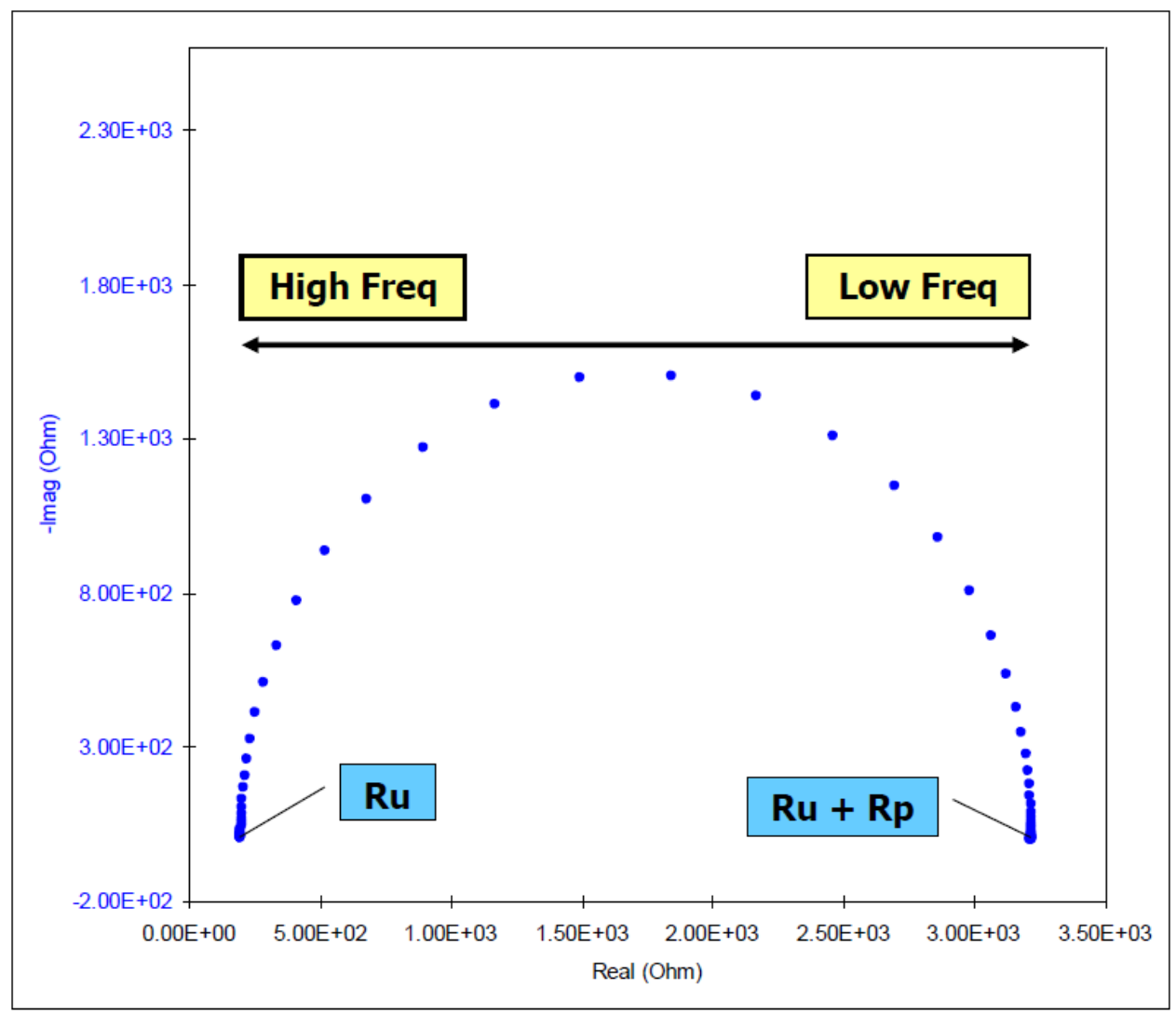

(b)

Figure 2-17: EIS spectra of (a) Bode plot and (b) Nyquist plot [24]

\subsubsection{Equivalent circuit modelling}

Corrosion of metals often behaves like a circuit, thus EIS can be used to create an equivalent circuit model to physically represent the actual measured system. If the model is a good representation of the actual measured system, then the changes/variations can be accounted for as a function of time [25]. In more simpler terms if a test specimen can form an oxide layer, then the equivalent circuit model can be used to further understand whether the surface displays resistive, capacitive or inductive behaviour or a combination of them all.

For a specimen to behave like an ideal resistor, an AC current sine wave will be the same as the sine wave voltage applied with $0^{\circ}$ phase angle. This would mean that there is no lag time between 
the voltage and current sine wave where the specimen behaves like a resistor that instantaneously reacts to any changes made in the voltage applied. The impedance term is represented by a real and imaginary vector in complex coordinates. Since the phase angle is $0^{\circ}$ for all frequencies it can be expressed as [26]:

$$
\begin{gathered}
Z_{\text {resistor }}=Z_{\text {real }}+j Z_{\text {imaginary }} \\
Z_{\text {resistor }}=R+j 0 \\
Z_{\text {resistor }}=R
\end{gathered}
$$

If the specimen surface behaves comparable to that of a resistor, then the phase angle will display a horizontal line since the phase angle is always $0^{\circ}$.

If a specimen behaves like an ideal capacitor, the current becomes frequency-dependent and it is $90^{\circ}$ out of phase compared the voltage. In this case, the impedance is represented with a straight line with a slope of -1 and the phase angle approaches at $-90^{\circ}$. The capacitor impedance term is expressed as [26]:

$$
\begin{gathered}
Z_{\text {capacitor }}=Z_{\text {real }}+j Z_{\text {imaginary }} \\
Z_{\text {capacitor }}=0+j \frac{-1}{2 \pi f C} \\
Z_{\text {capacitor }}=\frac{1}{j \omega C}
\end{gathered}
$$

Applying the same principle, if a specimen behaves like an ideal inductor, then the current varies linearly with frequency, which leads to a phase angle of $+90^{\circ}$ and the impedance has a slope of +1 . The impedance of an inductor is expressed as [26]: 


$$
\begin{gathered}
Z_{\text {inductance }}=Z_{\text {real }}+j Z_{\text {imaginary }} \\
Z_{\text {inductance }}=0+j \omega L \\
Z_{\text {inductance }}=j \omega L
\end{gathered}
$$

If a specimen has a surface that does not behave like a resistor, capacitor or an inductor, then it is termed as a non-ideal capacitor. The term, constant phase element (CPE), is used to describe a real inhomogeneous or an imperfect passive layer of an alloy, which is a non-ideal capacitor. The impedance expression for $\mathrm{CPE}$ is given as [26]:

$$
Z_{C P E}=\frac{1}{Q(j \omega)^{n}}
$$

Where the exponent, $n$, is an adjustable parameter between -1 and 1 . When $n=1$, the CPE is an ideal capacitor and the $Q$ constant is equal to the capacitance $(Q=C)$. For $n=-1, \mathrm{CPE}$ is an ideal inductor and for $n=0 \mathrm{CPE}$ is a resistor. For $0.5<n<1$, the CPE is described as pseudo-capacitance where the specimen has an imperfect passive layer. When $n=0.5$, this is described as Warburg impedance, which means current flow is limited by ionic diffusion, leading to a distinct $45^{\circ}$ angle tail line in the Nyquist plot in the low-frequency region $[25,26]$.

Figure 2-18(a) represents the equivalent circuit for a perfectly coated material, which usually has a high impedance. The resistance $R$ represents the solution resistance of the electrolyte and $C$ represents the capacitance of a coating/oxide layer [26]. Figure 2-18(b) shows an equivalent circuit model known as Randles cell, which is commonly used in electrochemistry and is referred to as the R-CPE model. It includes solution resistance of the electrolyte $\left(R_{s}\right)$, double layer capacitance or coating/oxide layer capacitance $\left(C_{d l}\right)$ and charge transfer resistance/parallel resistance $\left(R_{c t}\right)$ [26]. 


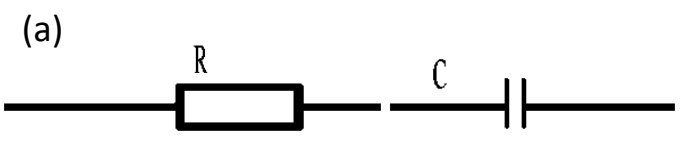

(b)

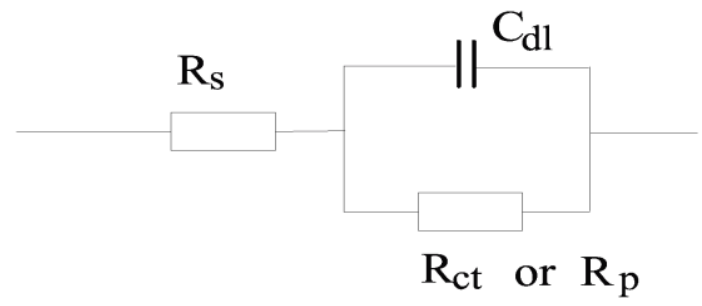

Figure 2-18: Equivalent circuit model: (a) a pure capacitor and (b) Randles circuit for an incomplete capacitor [26]

Solution resistance $\left(R_{S}\right)$ is the resistance between the WE and RE and is a major factor in measuring impedance in an electrochemical cell. If $R_{S}$ is large in value, then it would significantly contribute to impeding charge transfer as it will display a horizontal line for a broader range at higher frequencies. A low value of $R_{s}$ would not contribute much to impedance where the initial horizontal line will only display for a small range at higher frequencies. Double layer capacitance $\left(C_{d l}\right)$ exists between the alloy and the electrolyte solution, as shown in Figure 2-19. It is formed as a layer of ions when the electrolyte solution is absorbed on the alloy surface. The alloy surface and the ions are separated by an insulating space, which forms a capacitive layer allowing an alloy in an electrolyte solution to behave as a capacitor [26]. This concept is similar also for bare metals. The value of the double layer capacitance can be affected by various variables such as temperature, electrode roughness, impurities in solution, an oxide layer, electrode potential, etc. [26]. In the case where a coating/oxide layer exists on the surface of an alloy, the $C_{d l}$ abbreviation is changed to CPE. The charge transfer resistance $\left(R_{c t}\right)$ represents the ionic charge resistance or the rate of corrosion reactions at the OCP $[18,25]$. When a coating/oxide layer exists, then the $R_{c t}$ abbreviation is changed to $R_{p}$. If a coating/oxide layer on a specimen surface is strong and stable, it can have a high resistance value beyond $10^{8} \Omega$. 


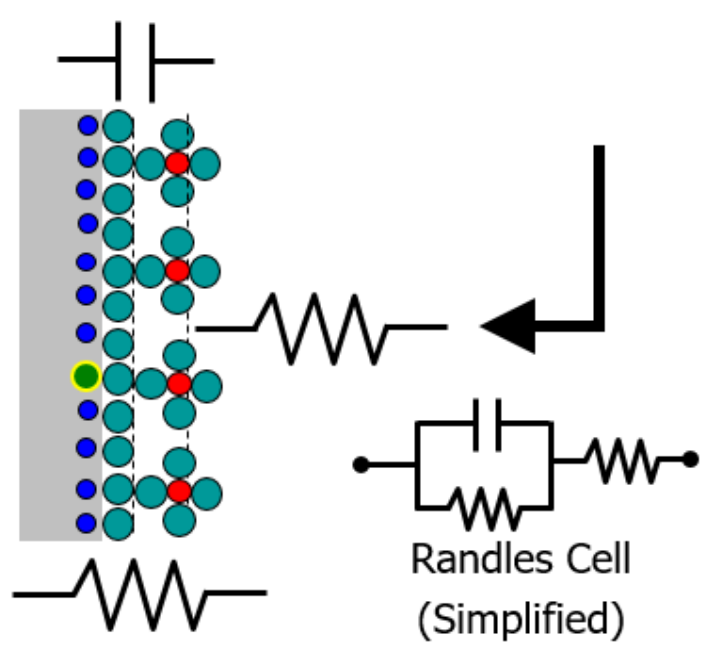

Figure 2-19: Schematic representation of the simplified Randles circuit [24]

Since an alloy surface can never realistically have a pure capacitance behaviour, the typical Randles circuit in Figure 2-18(b) can be used as a basis to define a heterogeneous specimen surface. The Bode plot for this reference example is shown in Figure 2-20, indicating a unique type of behaviour from high to low frequency. The impedance of the resistor component of a circuit is usually constant and the impedance of a capacitor is frequency-dependent. At high-frequency values, the impedance due to the capacitance of a coating/oxide layer decreases and at some point it will become smaller than $R_{s}$, thus it is possible that in the high-frequency region between $10^{3}$ $10^{6}$ the Randles circuit in Figure 2-18(b) is controlled entirely by $R_{s}$. As the frequency decreases the impedance increases due to increasing capacitive behaviour of the specimen surface, which represents the negative one slope line and is displayed to have a negative phase shift. In the lowfrequency region, the specimen behaves like a resistor once again and displays a horizontal line as the capacitance decreases. 

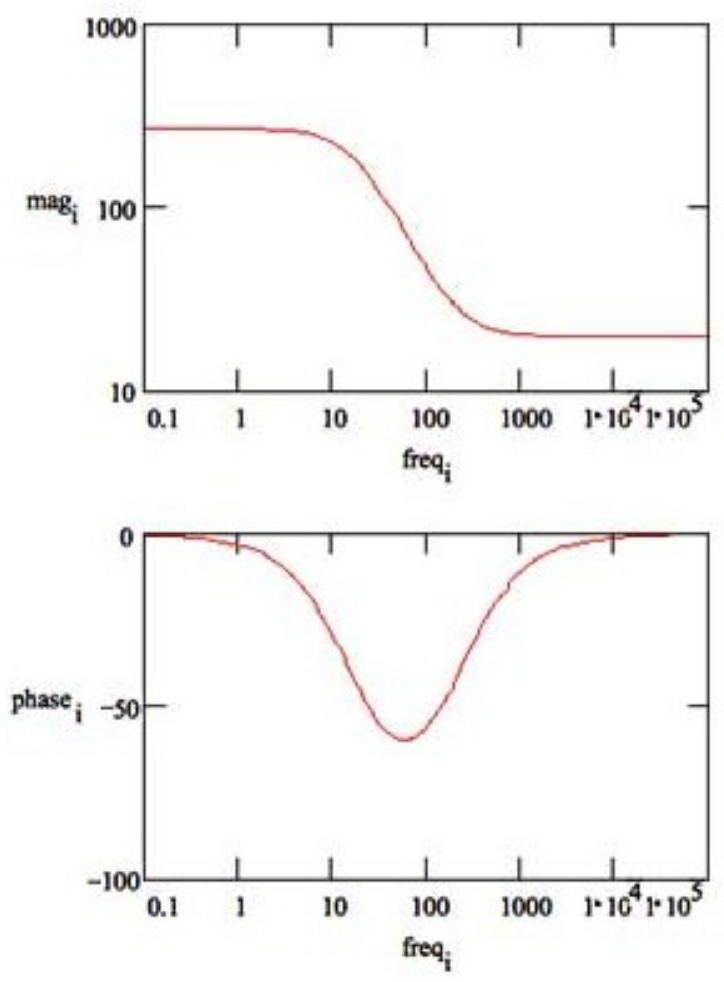

Figure 2-20: An example of bode plot for the reference Randles circuit [26]

For the example given in Figure 2-20, a horizontal line at higher frequencies would mean that solution resistance is higher than the impedance produced by the coating/oxide layer capacitance. As the frequency becomes lower, the capacitance portion is observed when the impedance becomes large and the sloped line starts approaching $-90^{\circ}$. In this case, the sloped line only makes it to $-50^{\circ}$ before switching back to a resistive mode with phase angle closer to $0^{\circ}$. This would be an indication that ions from the electrolyte have diffused into the oxide layer, meaning that capacitive behaviour is broken and the pore/crack resistance within the oxide layer plays a role [25]. Not all alloys will have similar behaviour to the example given, but the physical meaning behind each line remains the same. 


\subsubsection{Potentiodynamic polarization}

Potentiodynamic polarization or often referred to as cyclic polarization is a test method used to determine general corrosion behaviour using corrosion potential $\left(E_{c o r r}\right)$, corrosion current density $\left(I_{c o r r}\right)$ and polarization resistance $\left(R_{p}\right) . E_{c o r r}$ and $I_{c o r r}$ are values that define the point where corrosion of an alloy effectively starts and the $R_{p}$ value indicates the resistance to corrosion that is taking place. Figure 2-21 shows a hypothetical polarization forward curve of an alloy, where all the different types of characteristics of a polarization curve are displayed. Depending on the type of the alloy in question, the plots will be similar, but will not display the exact behaviour. The polarization scan can be started from a designated starting point specified in a computer software and can be set to run at a maximum specified potential value at a specific scan rate. This test is performed by applying DC voltage to the alloy surface.

The test starts with a slow increase in potential to monitor the response of current, thus formulating a cathodic region and eventually reaching a measured current close to zero where the cathodic and anodic reaction rates are equal. This equilibrium region between the anode and cathode is where the general corrosion parameters are obtained. Beyond the turning point, continuously increasing the potential makes the current rise to the active region where the oxidation reaction is dominant. As the potential continues to increase, a point is reached where the current almost does not change, indicating a passivation region on the curve. This is due to oxide film formation on the specimen surface where corrosion is being resisted. A transpassive region is eventually formed beyond the pitting potential $\left(E_{p}\right)$ where passive film breakdown and recovery takes place simultaneously. The parameters $E_{c o r r}$ and $I_{c o r r}$ can be obtained from Tafel extrapolation [21], which are important parameters characterizing the corrosion behaviour of an alloy. 


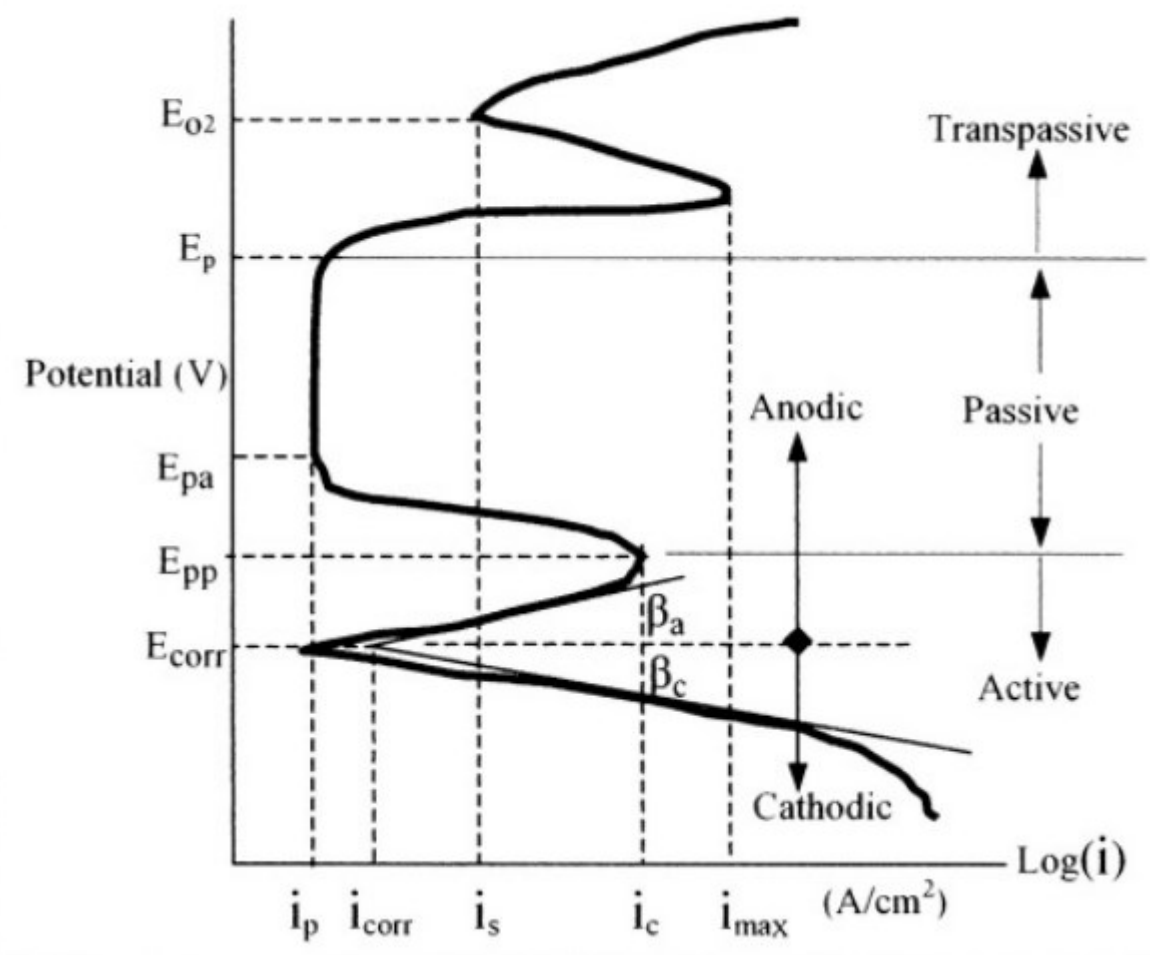

Figure 2-21: Hypothetical polarization curve [10, 22]

$I_{\text {corr }}$ is obtained based on the Stern-Geary (SG) relationship, which is a coefficient determined from both anodic and cathodic Tafel slopes [21]:

$$
\begin{gathered}
B=\frac{\beta_{a} \beta_{c}}{2.303\left(\beta_{a}+\beta_{c}\right)} \\
I_{c o r r}=\frac{\beta_{a} \beta_{c}}{2.303\left(\beta_{a}+\beta_{c}\right) R_{p}}
\end{gathered}
$$

The value of $R_{p}$ is obtained using Eq (2) where it is the inverse of $I_{\text {corr }}$ [21]:

$$
R_{p}=\frac{B}{I_{\text {corr }}}
$$


The SG coefficient can be calculated from the Tafel slopes, which are the corresponding $\beta_{a}$ and $\beta_{c}$ values shown in Figure 2-21, where both anodic and cathodic reactions strictly obey Tafel kinetics and their reactions are distributed uniformly over the specimen surface. The polarization curves can be further quantified with calculating the corrosion rate. The following relationships can be used for both potentiodynamic and potentiostatic analyses. Equivalent weight is first determined, which is defined as the mass of metal that is oxidized by one Faraday of electric charge and is given as [21]:

$$
Q=\sum \frac{n_{i} f_{i}}{W_{i}}
$$

where $n_{i}=$ valence of the $i^{\text {th }}$ element in the metal, $f_{i}=$ mass fraction of the $i^{\text {th }}$ element, $W_{i}=$ atomic weight of the $i^{\text {th }}$ element, and $Q$ is the electron equivalent for $1 \mathrm{~g}$ of the metal. Thus, the equivalent weight is the reciprocal of $Q$ given as [21]:

$$
E W=\frac{1}{Q}
$$

With density, corrosion current density and equivalent weight obtained, the corrosion rate is calculated using the following Faraday's law expression [21]:

$$
C R=K_{1} \frac{I_{\text {corr }}}{\rho} E W
$$

The value of $K_{l}$ is a constant used in calculating the corrosion rate, which is measured in $\mathrm{mm} / \mathrm{yr}$. and $\rho$ is the metal density. Mass loss rate can also be calculated as an alternative using the following expression [21]: 


$$
M R=K_{2} I_{c o r r} E W
$$

$K_{2}$ is a constant and the mass loss rate is measured in $\mathrm{mg} / \mathrm{dm}^{2} \mathrm{~d}$.

\subsubsection{Hysteresis loop}

Cyclic polarization often involves plotting a backward curve measurement where the potential is scanned backward to qualitatively determine an alloys localized corrosion behaviour. This backward scan is called a hysteresis loop and it is a method to determine an alloys ability to resist pitting. The backward scan is started from the onset of the peak potential value and the localized corrosion behaviour is observed only near the peak potential values. There are essentially three different ways to characterize the hysteresis loop. First, is if the reverse scan displays an electronegative hysteresis meaning the backward scan has larger current density compared to forward scan at the same potential. This would indicate that the specimen has clear signs of deep pitting corrosion susceptibility [23]. Second, is if the backward scan is extremely close to forward scan by a small margin. This is known as stable hysteresis where the specimen can display very small pits at higher potential values but is more resistant to the initiation and propagation of localized corrosion [23]. Third, is where the current density backward is smaller compared to current density forward. This is known as electropositive hysteresis where the specimen entirely goes through uniform corrosion behaviour beyond its corrosion potential and does not display any signs of pitting behaviour [23]. Figure 2-22 shows an example of how negative and stable hysteresis cases look like. Stainless steel type 304 displays a large negative hysteresis while alloy C-276 display stable hysteresis near peak potential values. 


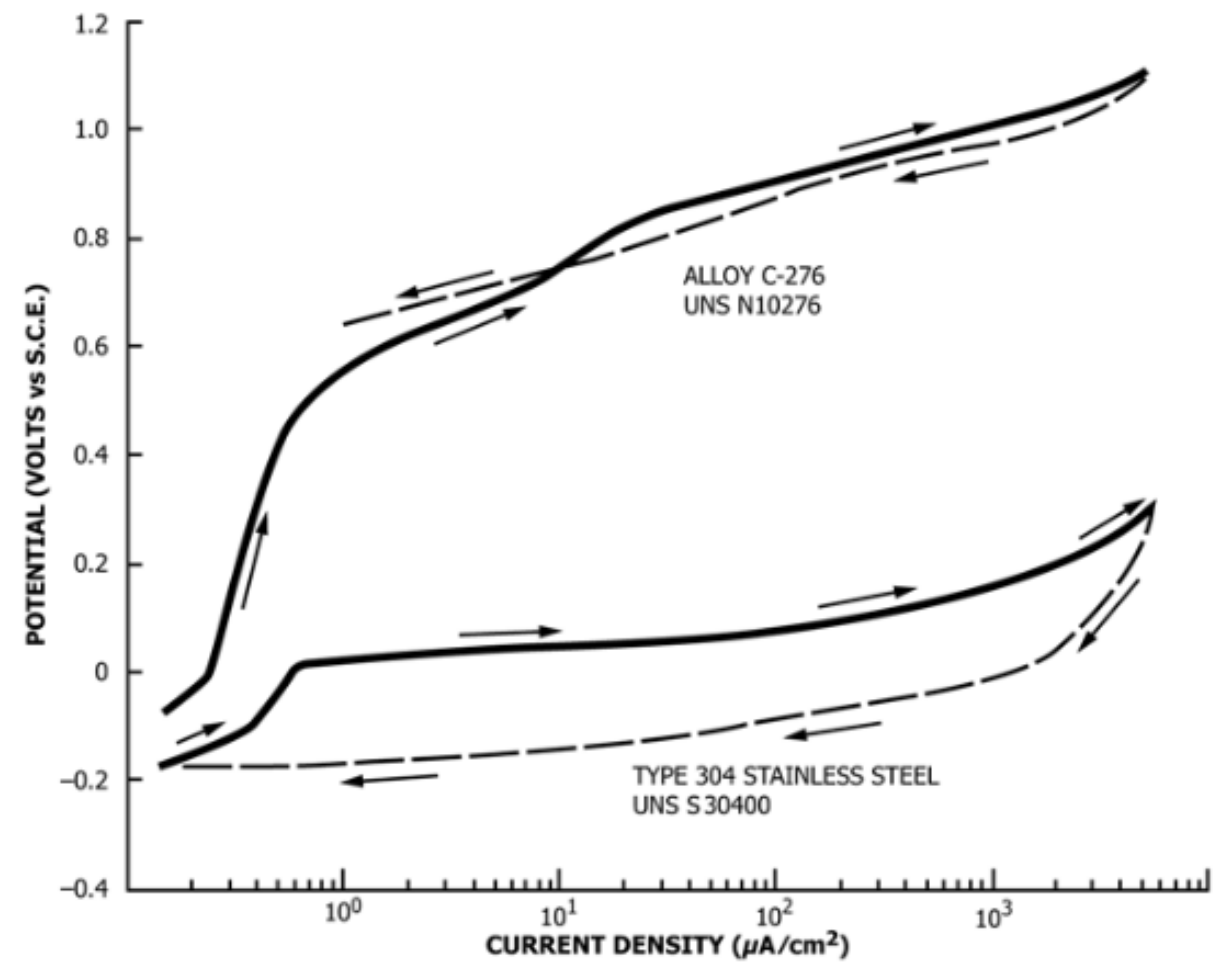

Figure 2-22: Cyclic polarization curves displaying hysteresis loop (dotted lines) [23]

\subsubsection{Potentiostatic polarization}

Potentiostatic polarization is also another experimental technique used to determine $I_{\text {corr }}$ with the DC voltage input locked at a specified value such as $0.5 \mathrm{~V}, 1 \mathrm{~V}$, etc. With the voltage kept constant for a set amount of time, current density behaviour is monitored where it eventually reaches a stable state. This stable state can be considered as $I_{\text {corr }}$ for the specified constant voltage. Potentiostatic test historically has not been a test method that is used or explored to characterize an alloys corrosion behaviour, however it can be used to investigate current behaviour as the alloy undergoes severe oxidation at higher potentials. 


\section{Chapter: Experimental Details}

\subsection{Alloy Specimens}

Three as-cast 700 series Stellite alloys, designated as Stellite 706, Stellite 712 and Stellite 720, were studied in this research. The alloy specimens were provided by Kennametal Stellite Inc., with the detailed chemical compositions given in Table 3-1. Their compositions are similar except for the contents of the alloying elements $\mathrm{C}$ and Mo varying among the alloys. Stellite 706 has the least amounts of C and Mo, while Stellite 720 has the most.

Table 3-1: Chemical compositions (wt.\%) of 700 series Stellite alloys $[1,3]$

\begin{tabular}{|c|c|c|c|c|c|c|c|c|}
\hline Alloy & Co & Cr & Mo & C & Fe & Ni & Si & Mn \\
\hline Stellite 706 & Bal. & 29 & 5 & 1.2 & $3(\max )$ & $3(\max )$ & $1.5(\max )$ & $1.5(\max )$ \\
\hline Stellite 712 & Bal. & 29 & 8.5 & 2 & $3(\max )$ & $3(\max )$ & $1.5(\max )$ & $1.5(\max )$ \\
\hline Stellite 720 & Bal. & 33 & 18 & 2.5 & $3(\max )$ & $3(\max )$ & $1.5(\max )$ & $1.5(\max )$ \\
\hline
\end{tabular}

The microstructures of the alloy specimens were examined with SEM using the Tescan Vega-II $\mathrm{XMU}$, as shown in Figure 3-1. The SEM images at a low and high magnification of the microstructures are presented in Figure 3-2 to Figure 3-4. These alloys have a similar microstructure consisting of $\mathrm{Cr}_{7} \mathrm{C}_{3}$ carbide in black and $(\mathrm{Co}, \mathrm{Mo})_{3} \mathrm{C}$ carbide in white embedded in CoCr solid solution matrix in grey.

For Stellite 706, there are major dendritic $\mathrm{Cr}_{7} \mathrm{C}_{3}$ carbide and minor $(\mathrm{Co}, \mathrm{Mo})_{3} \mathrm{C}$ carbide distributed across the CoCr solid solution matrix, see Figure 3-2. For Stellite 712, with the increase in C and Mo contents, the amount of $\mathrm{Cr}_{7} \mathrm{C}_{3}$ and $(\mathrm{Co}, \mathrm{Mo})_{3} \mathrm{C}$ carbide increases, as seen in Figure 3-3. With greater $\mathrm{C}$ and Mo contents result in significant increase in $(\mathrm{Co}, \mathrm{Mo})_{3} \mathrm{C}$ carbides, as seen in Figure 3-4 for Stellite 720. The size of $\mathrm{Cr}_{7} \mathrm{C}_{3}$ carbides in Stellite 720 become somewhat large spherical shape displayed by the lower magnification image in Figure 3-4(a). The dark grey regions consist of more $\mathrm{Cr}$ and light grey region consists of more Co. 
The volume fractions of carbides in these three alloys are taken from previous literature [11]. They were estimated using image analysis and the data are reported in Table 3-2. Stellite 706 has the least amounts of $\mathrm{C}$ and Mo among the three alloys, hence its carbide volume fraction is the lowest. With Stellite 712 and Stellite 720 having more C, more carbides are formed in these alloys. Stellite 720 contains approximately 18 wt. $\%$ Mo so that it has the highest volume fraction of Mo-rich carbides.

Table 3-2: Carbide volume fraction of 700 series Stellite alloys [11]

\begin{tabular}{|c|c|c|c|}
\hline Alloy & CoCr solid solution (\%) & Cr-rich carbide (\%) & Mo-rich carbide (\%) \\
\hline Stellite 706 & 83.45 & 13.91 & 2.64 \\
\hline Stellite 712 & 70.36 & 24.26 & 5.38 \\
\hline Stellite 720 & 55.31 & 25.09 & 19.6 \\
\hline
\end{tabular}

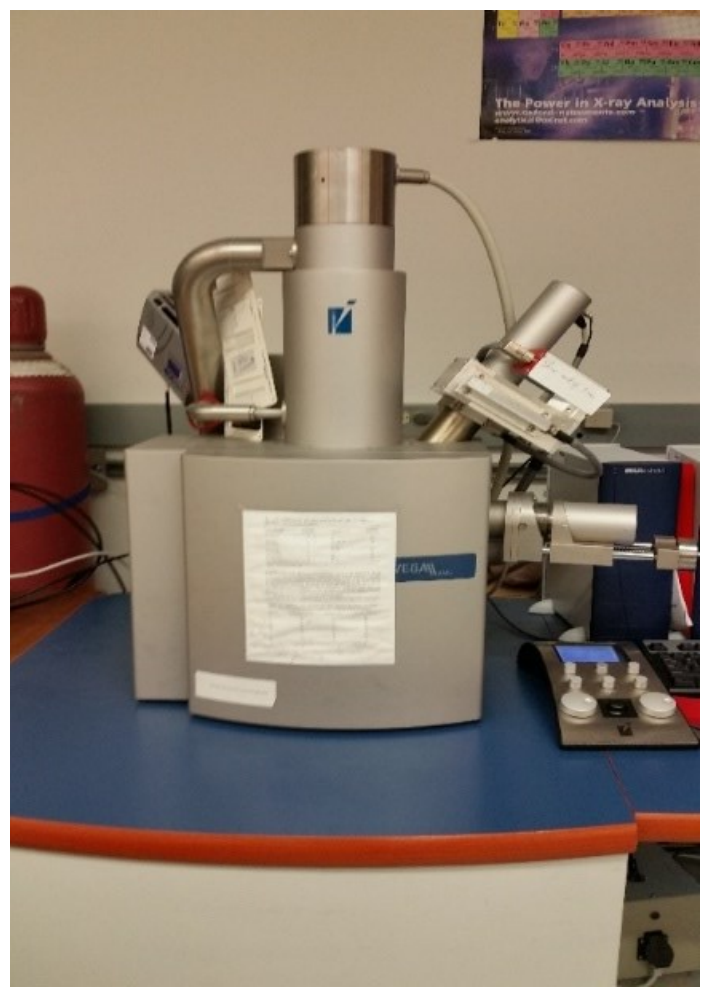

Figure 3-1: Tescan Vega-II XMU SEM instrument 


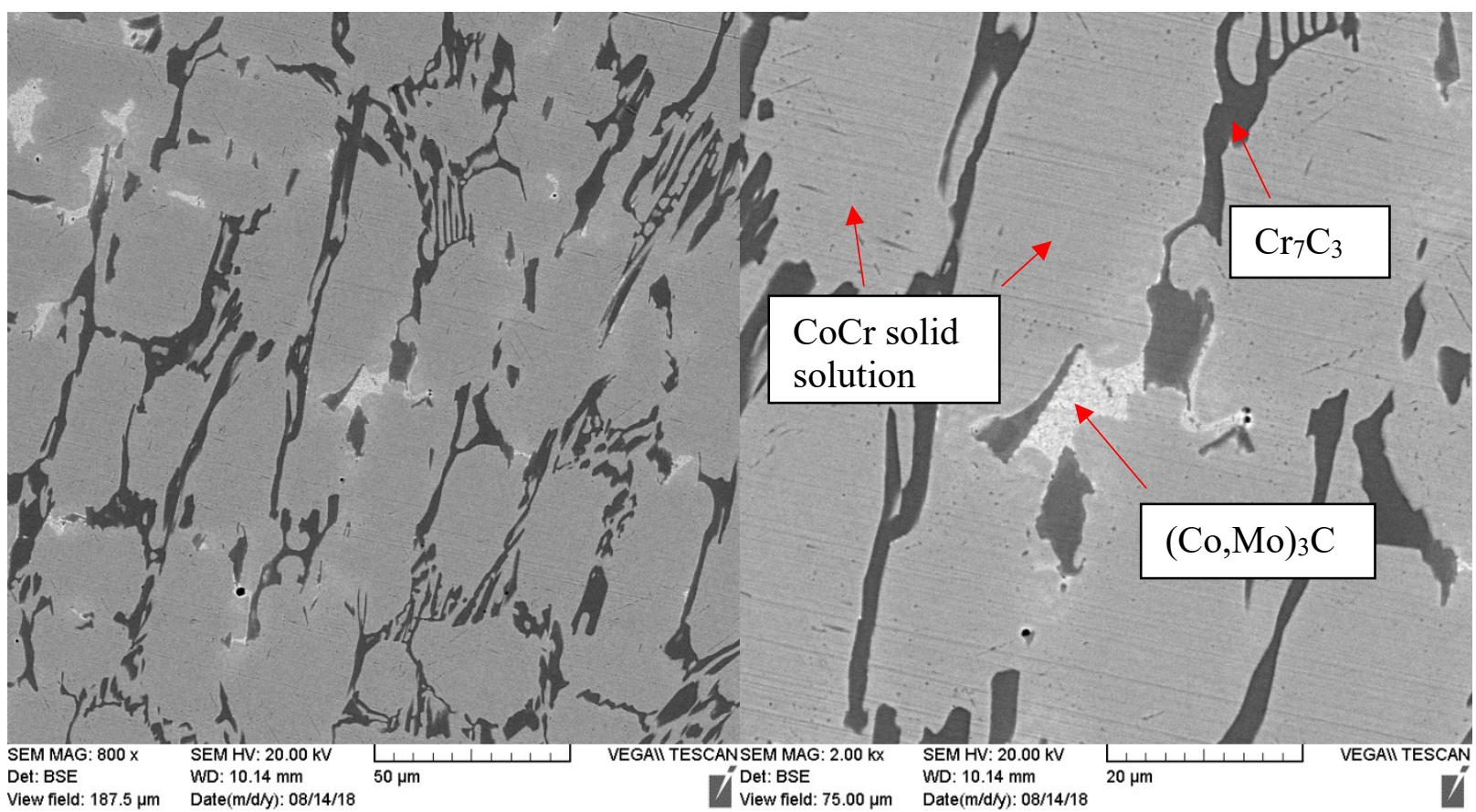

a)

b)

Figure 3-2: SEM microstructure of Stellite 706 a) 800x b) 2000x

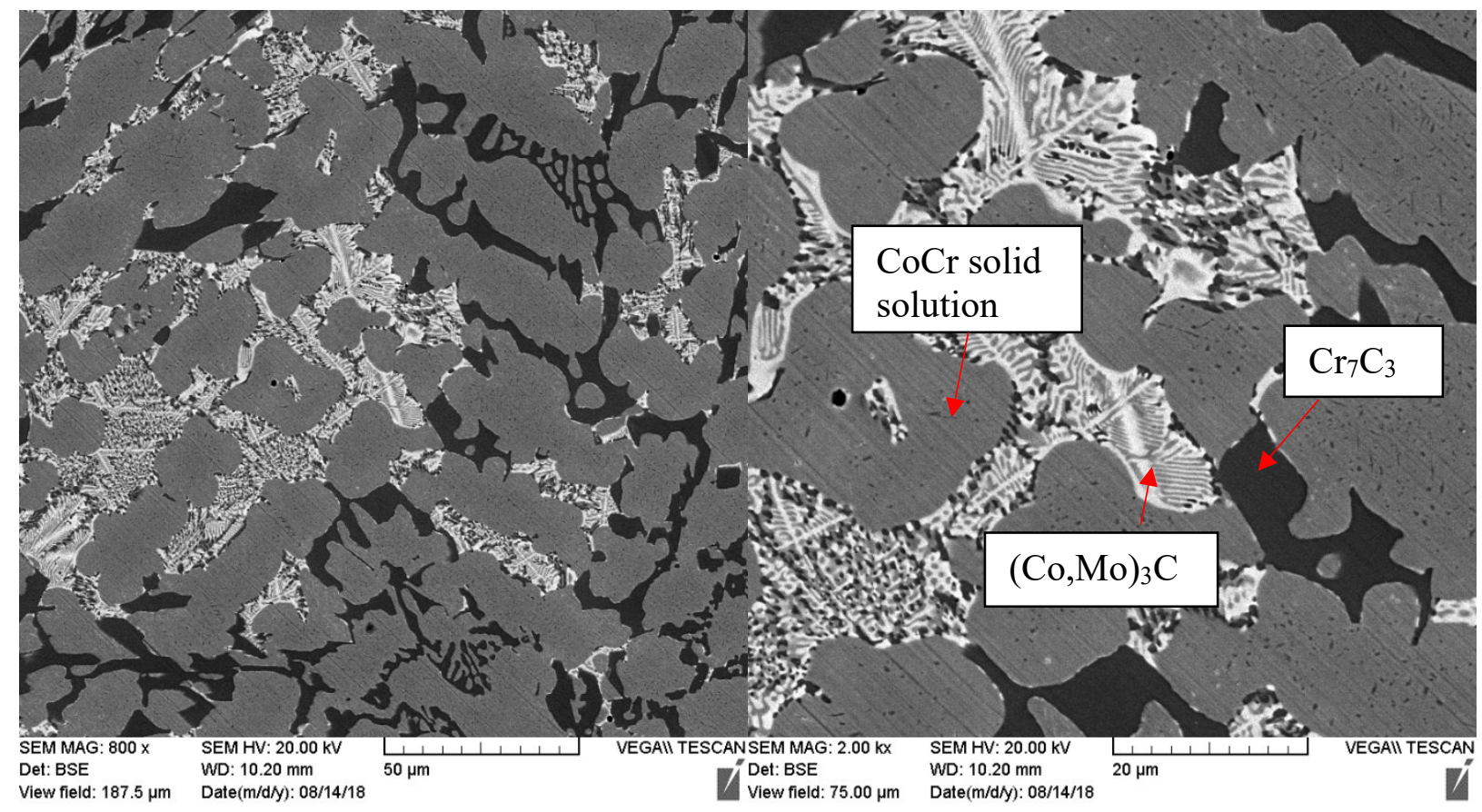

a)

b)

Figure 3-3: SEM microstructure of Stellite 712 a) 800x b) 2000x 


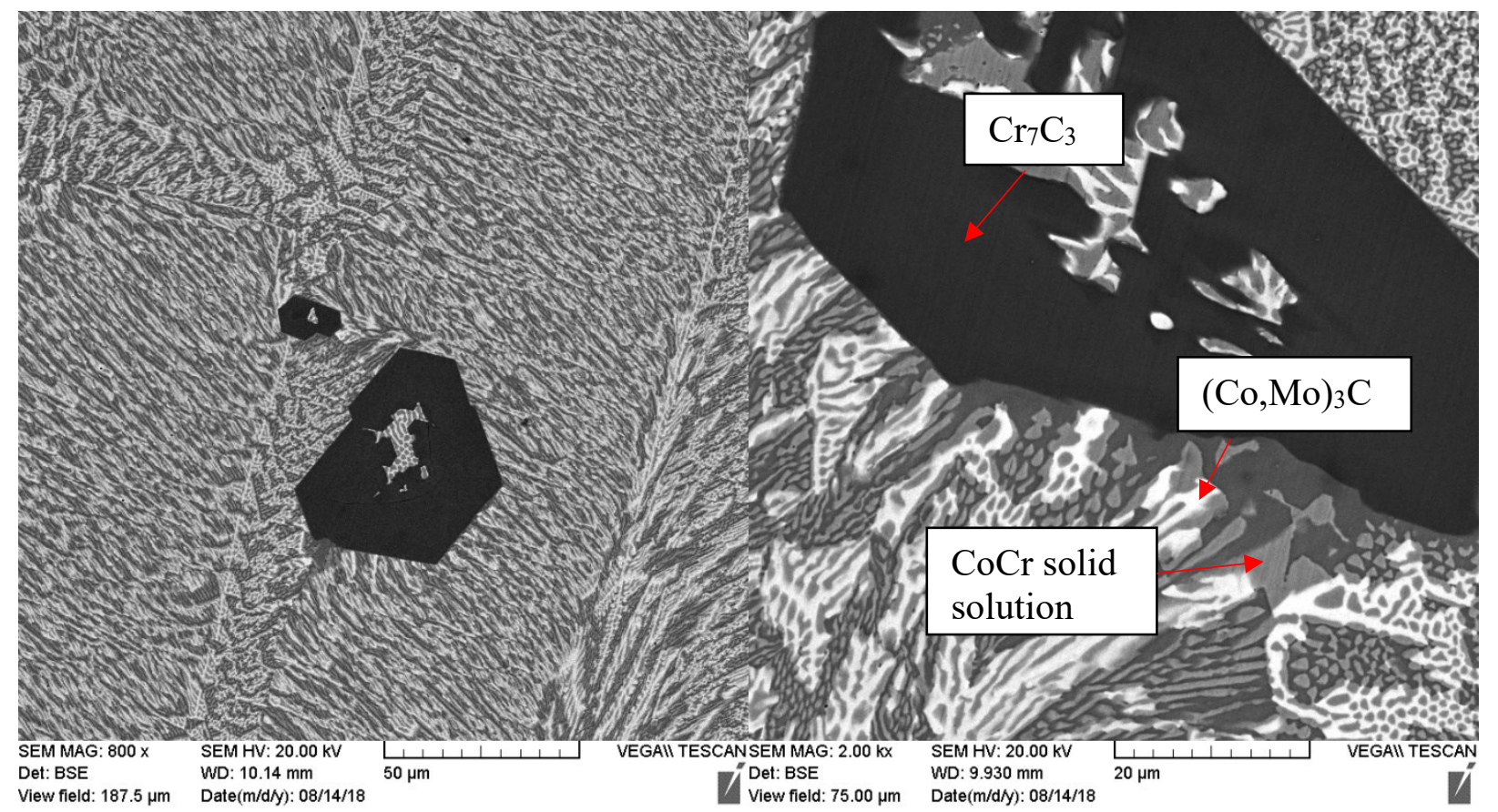

a)

b)

Figure 3-4: SEM microstructure of Stellite 720 a) 800x b) 2000x

All corrosion test specimens were prepared using the conventional grinding and polishing process, where they were attached on a cylindrical mounting rod via sticky tape. Silicon carbide water cooled grit papers from 400 to 1200 were used to grind the specimens, followed by the $9 \mu \mathrm{m}$ and $3 \mu \mathrm{m}$ polish finish. Polishing was not necessarily needed for polarization and EIS tests, but it can be done to achieve a smoother surface. Each grinding step was done manually, which included rotating the specimens $90^{\circ}$ after each subsequent grinding papers to eliminate scratches [30]. For each grinding paper, a rotating speed of $300 \mathrm{rpm}$ was used under approximately $27 \mathrm{~N}$ force until a planar surface was achieved [31]. The polishing step required the specimen to be held for approximately two minutes at a rotating speed of $150 \mathrm{rpm}$ under approximately $27 \mathrm{~N}$ force to obtain a reflective surface [31]. Each specimen was rinsed with tap water after each grinding step to clear excess debris from the surface, rinsed after final polishing and blow dried to quickly 
remove water from the surface to prevent stains. Each specimen was also rinsed with acetone before the corrosion tests to ensure that the surface was free from any debris.

\subsection{Electrolyte Solution}

The electrolyte solution for the experiments was a mixture of $700 \mathrm{ml}$ de-ionized water with 180 $\mu \mathrm{L}$ morpholine to obtain a $\mathrm{pH}$ value of 9.5 using an appropriate pipette. A digital waterproof $\mathrm{pH}$ meter was used to measure the $\mathrm{pH}$ value after the solution had been properly mixed with a magnetic stirrer for approximately 30 seconds. The solution was made under a fume hood due to the corrosive and toxic nature of concentrated morpholine. Proper personal protective equipment (PPE) was used when preparing the solution, which included nitrile gloves, safety goggles, safety mask, and lab coat. The corrosion tests were conducted at two temperatures of $25^{\circ} \mathrm{C}$ and $50^{\circ} \mathrm{C}$ using an ANOVA water bath shown in Figure 3-5 to control the temperature conditions.

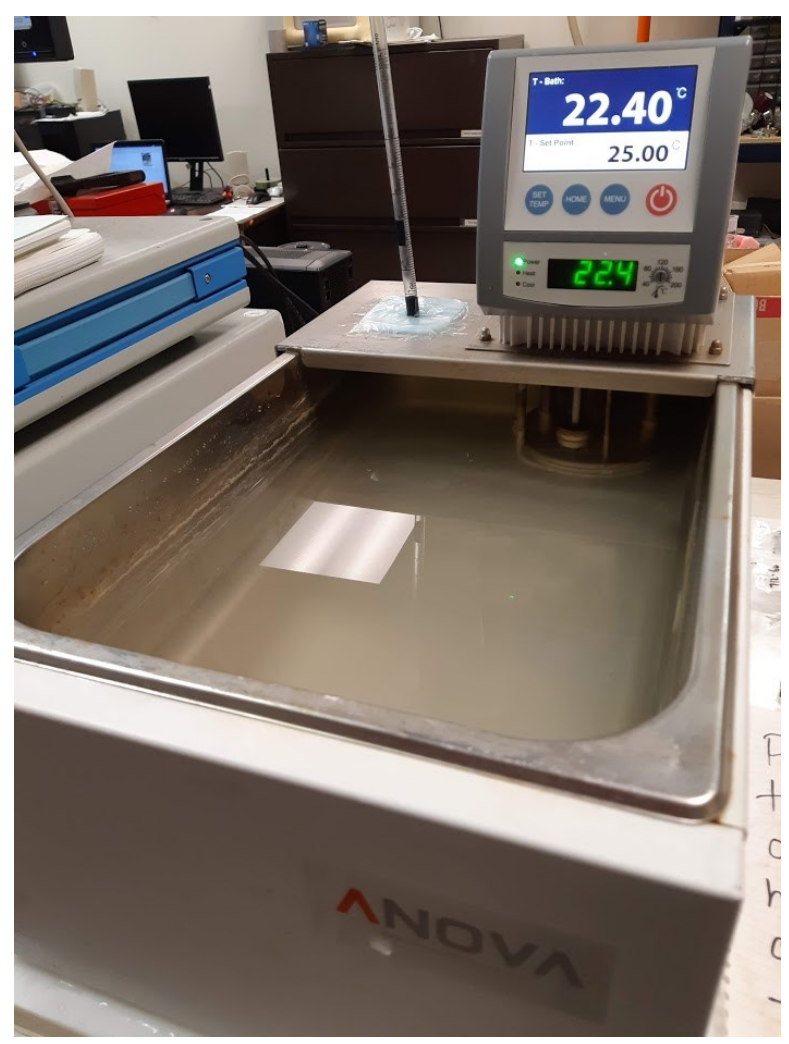

Figure 3-5: ANOVA water bath 


\subsection{Experimental Setup}

For corrosion testing, ASTM G5-14 [27] and ASTM G3-14 [28] were followed as instructions for conducting the polarization and EIS tests. The testing cell that is referenced from ASTM G5-14 is shown in Figure 3-6 where a similar three-electrode test cell was used to conduct all the experiments in this research. Figure 3-7 illustrates the overall test setup station with the various apparatus used for each experiment.

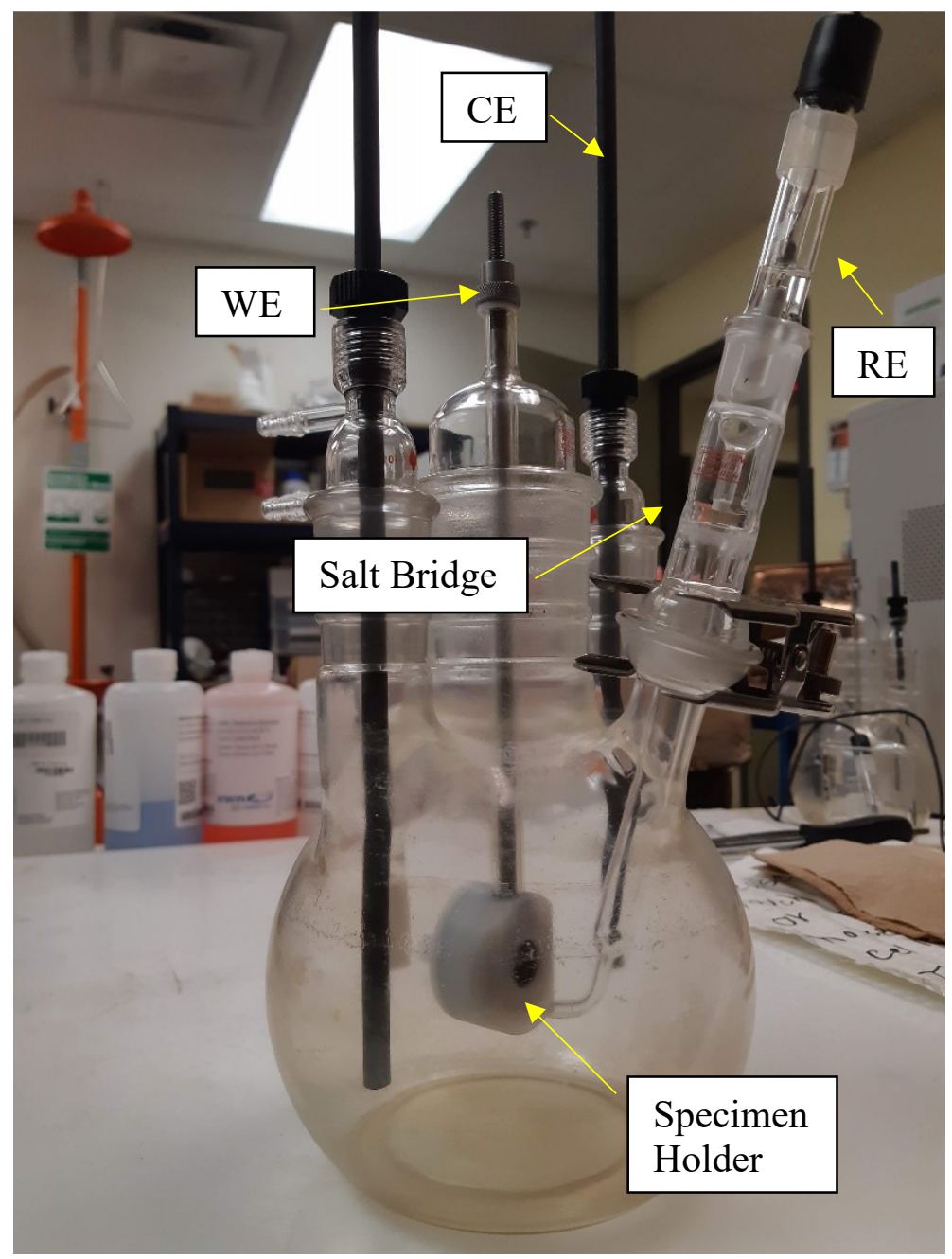

Figure 3-6: Corrosion test cell 


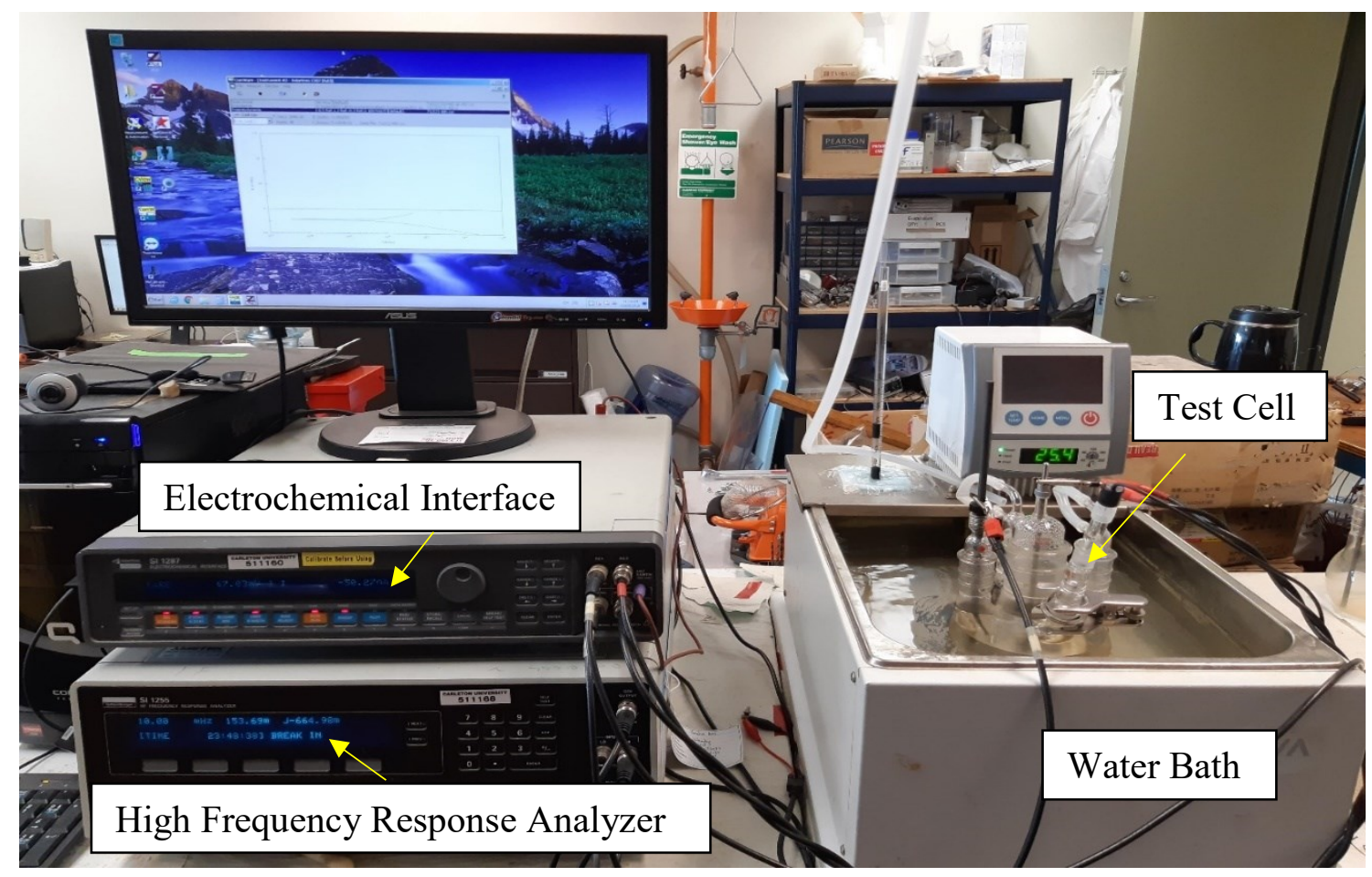

Figure 3-7: Corrosion test setup station

For both potentiodynamic and potentiostatic experiments, an instrument called Solartron SI 1287 Electrochemical Interface was used. EIS experiments were performed using the Schlumberger SI 1255 High Frequency Response Analyzer. The Solartron provided direct current (DC) for both polarization experiments and the software utilized to extract data is called CorrWare (version 3.2c) and CorrView (version 3.2d). For the EIS experiments, the software used is called ZPlot (version 3.2c) and ZView (version 3.2d). The corrosion cell is approximately a 1-liter flat bottom glass flask, which can withstand temperatures up to $100^{\circ} \mathrm{C}$ and is resistant to many solution types except for hydrofluoric acid solutions and extremely high $\mathrm{pH}$ solutions. The test cell is a three-electrode cell, which includes WE, CE and RE. The WE is the specimen being tested, which is $16 \mathrm{~mm}$ in diameter and $1.6 \mathrm{~mm}$ in thickness, mounted on a Teflon holder with a $12 \mathrm{~mm}$ diameter surface exposed to the electrolyte. The limitation of the WE sample holder is that it cannot be used for 
high-temperature testing beyond $50^{\circ} \mathrm{C}$ for a long period of time without the risk of damaging it. The CE was used to close the current circuit in order to balance the charge added or removed from the WE [29]. The $\mathrm{CE}$ is usually an inert material (Pt, Au, graphite, or glassy carbon) and does not participate in the electrochemical reactions taking place. The RE was used as a control medium to accurately measure and control the WE potential [29]. For this experiment, a high stable mercury RE was used, which is commonly known as saturated calomel electrode (SCE) due to pure mercury covering a platinum wire immersed in a saturated potassium chloride solution. The RE reaction is expressed as [29]:

$$
\mathrm{Hg}_{2} \mathrm{Cl}_{2}+2 e^{-} \rightarrow 2 \mathrm{Hg}+2 \mathrm{Cl}^{-} \quad E^{\circ}=0.244 V_{S H E} \text { at } 25^{\circ} \mathrm{C}
$$

where $E^{\circ}$ is the reference potential value at standard temperature and pressure. The standard hydrogen electrode (SHE) was used where all other types of RE can be expressed in terms of some constant deviation from SHE. The SHE reference reaction is the reduction reaction of hydrogen as follows [29]:

$$
2 \mathrm{H}^{+}+2 e^{-} \rightarrow \mathrm{H}_{2}(g) \quad E^{\circ}=0.000 V_{S H E} \text { at } 25^{\circ} \mathrm{C}
$$

This experiment must be in a chloride free environment and one may argue that using a potassium chloride SHE would be a poor choice due to a chance of chloride ions leaking into the bulk solution [29]. To counter this from happening a salt bridge was used, which contained a small portion of the bulk solution to prevent any leaks through the glass frit [29]. The salt bridge also acted to ensure that the RE and WE were close to one another without contact.

\subsection{Experimental Procedure}

Before starting any specific experiment, the electrolyte solution was purged with argon gas for 20 minutes at approximately 1.5 psi pressure. The purging was done to push the air out of the free 
surface and minimize dissolved oxygen concentration in the solution. Following the argon purge, open circuit potential (OCP) was conducted for about 1.5 hours to achieve an approximately steady-state in order to obtain accurate results of EIS. As a minimum standard, 1.5 hours OCP is used initially for both temperature and surface conditions. The EIS software parameters were set to use a small excitation signal of $10 \mathrm{mV}$ of $\mathrm{AC}$, which was applied to the specimen surface. In this research, the EIS spectra were performed from a high frequency of $10^{6} \mathrm{~Hz}$ down to a low frequency of $10^{-2} \mathrm{~Hz}$.

Cyclic polarization test was performed using a set of parameters in the setup program. The scan rate for all experiments was set to $0.1667 \mathrm{mV} / \mathrm{s}$ with the initial start voltage at negative $0.5 \mathrm{~V}$, peak voltage at $1.2 \mathrm{~V}$, and final voltage at negative $0.3 \mathrm{~V}$. For the failure experiments, the scan rate is set to $25 \mathrm{mV} / \mathrm{s}$ up to a peak potential of $12 \mathrm{~V}$, with the initial and starting voltage remaining the same.

For potentiostatic experiments, three different constant voltages were used such as $1 \mathrm{~V}, 2 \mathrm{~V}$ and 3 V for all Stellite alloys for up to one hour each.

\subsection{Wear Test}

To simulate the real industrial service condition of valve trim components, where there is a combination of corrosion and wear (erosion-corrosion), the surface of the test specimens was subjected to dry-sliding wear with a ball-on-disc tribometer before corrosion testing. The wear test was performed on three specimens of each alloy to investigate their corrosion behaviour. Polarization and EIS tests were conducted on the damaged surface specimens at both temperature conditions to study if their corrosion behaviour had any significant changes compared to the intact surface. The Neoplus ball-on-disc tribometer machine shown in Figure 3-8(a). The specimen was mounted to a square based static disk. A spherical tip ball of $2.5 \mathrm{~mm}$ radius, which was rotating at 
a speed of $120 \mathrm{rpm}$, was pressed down on the specimen surface under a load of $200 \mathrm{~N}$, illustrated in Figure 3-8(b). With the wear proceeding, a $3 \mathrm{~mm}$ diameter circular wear track was created on the specimen surface, as shown in Figure 3-8(c). The tip of the sphere is made of W carbide with a hardness value of $1534 \mathrm{HV}$. The duration of wear test on each specimen was 20 minutes at room temperature. The worn surfaces of the specimens are shown in Figure 3-9. From the SEM images, the Stellite 706 worn surface has deeper plow scars which result from abrasive wear and distinct white regions indicate adhesive wear due to friction [11]. Compared to Stellite 706, the worn surface of Stellite 720 was less damaged because the wear track is shallow. In fact, it is not significant as to how much the specimens were worn or the time set for this experiment, but rather to see if the polarization and EIS qualitative and quantitative data of worn alloys would display different results compared to intact specimens. In application, erosion-corrosion damage for valve trims is usually abrasive wear. However, whenever there is wear involved the combination of abrasive and adhesive wear is always present at the same time. Only one type of wear can be simulated here as it is not possible to model all wear criteria at the same time due to instrument limitations. By performing corrosion test on the worn Stellite samples at two different temperature conditions, it will give an idea of whether the performance of damaged specimens differs compared to intact. 


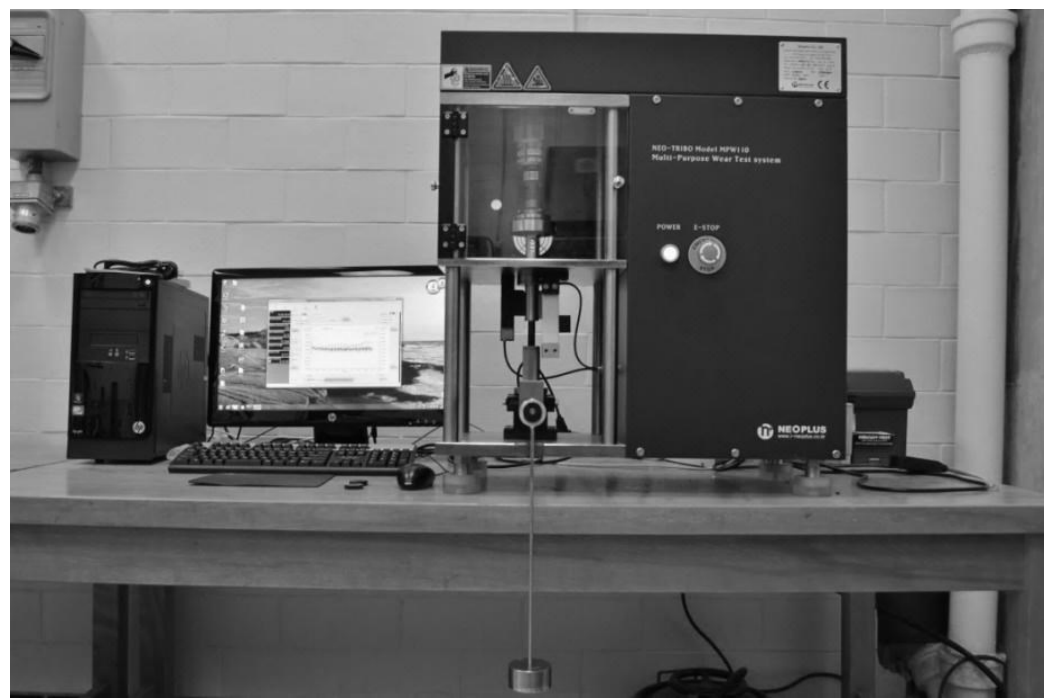

(a)

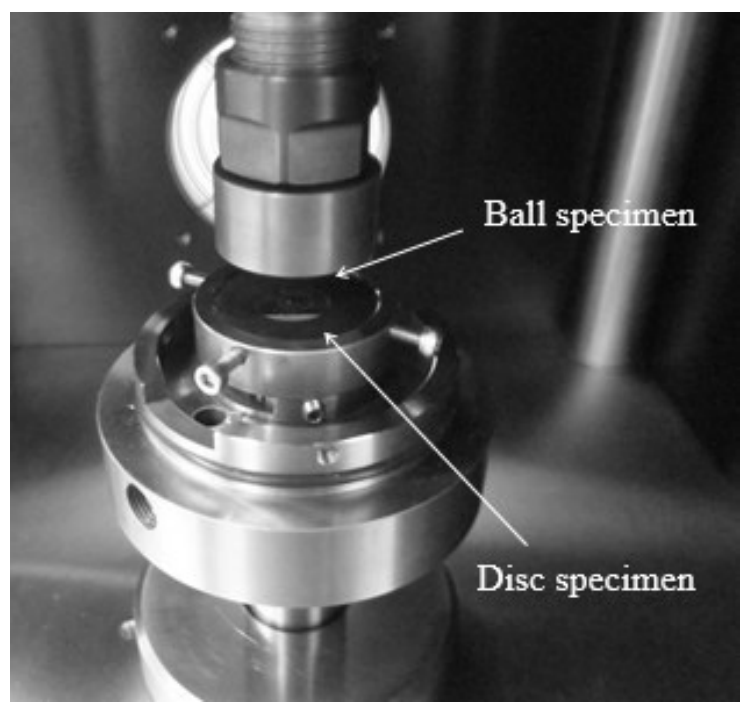

(b)

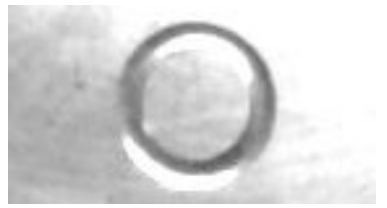

(c)

Figure 3-8: Ball-on-Disc Tribometer: (a) overall setup, (b) specimen holder and (c) photographic wear track generated in specimen surface 


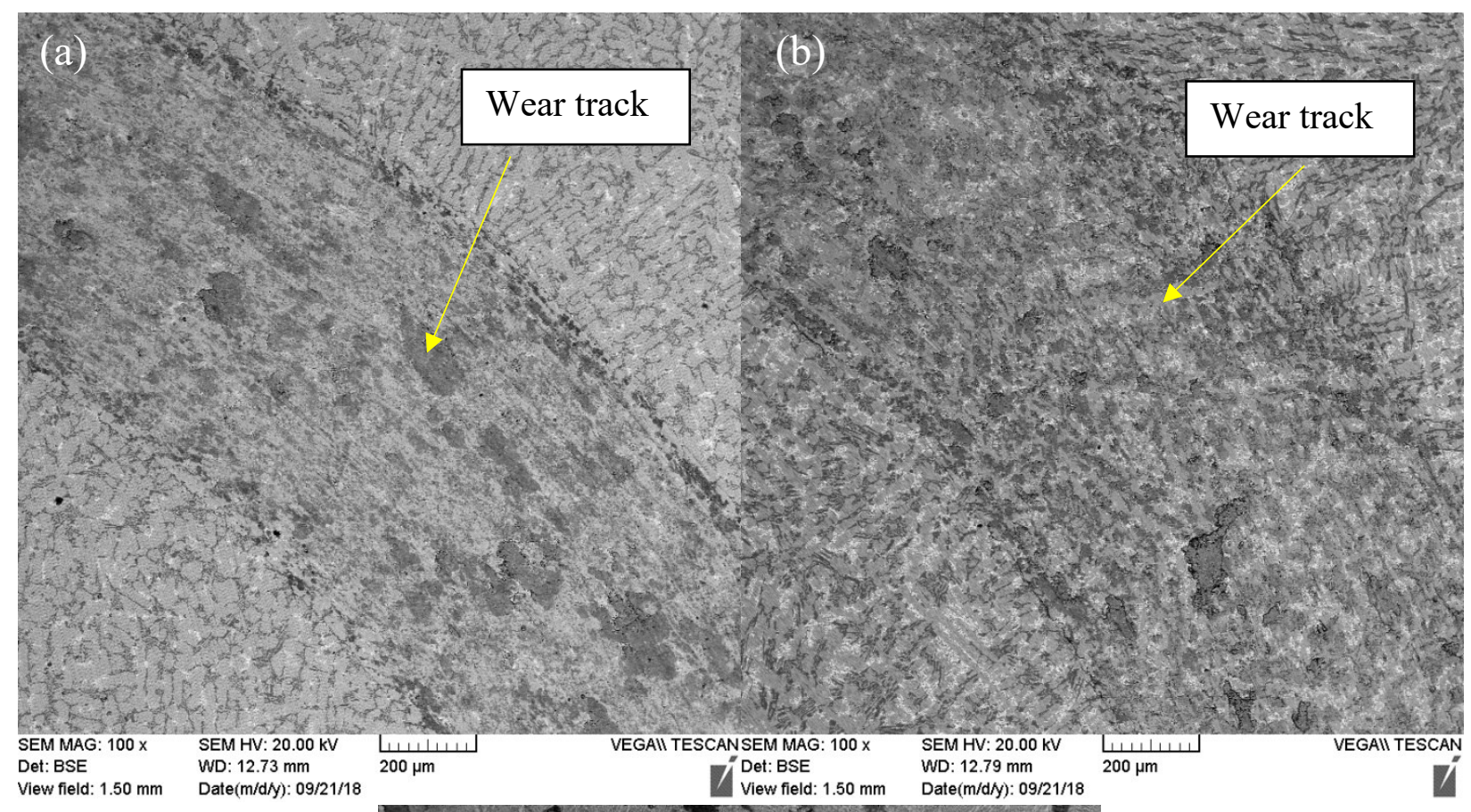

Date(m/d/y): 09/21/18

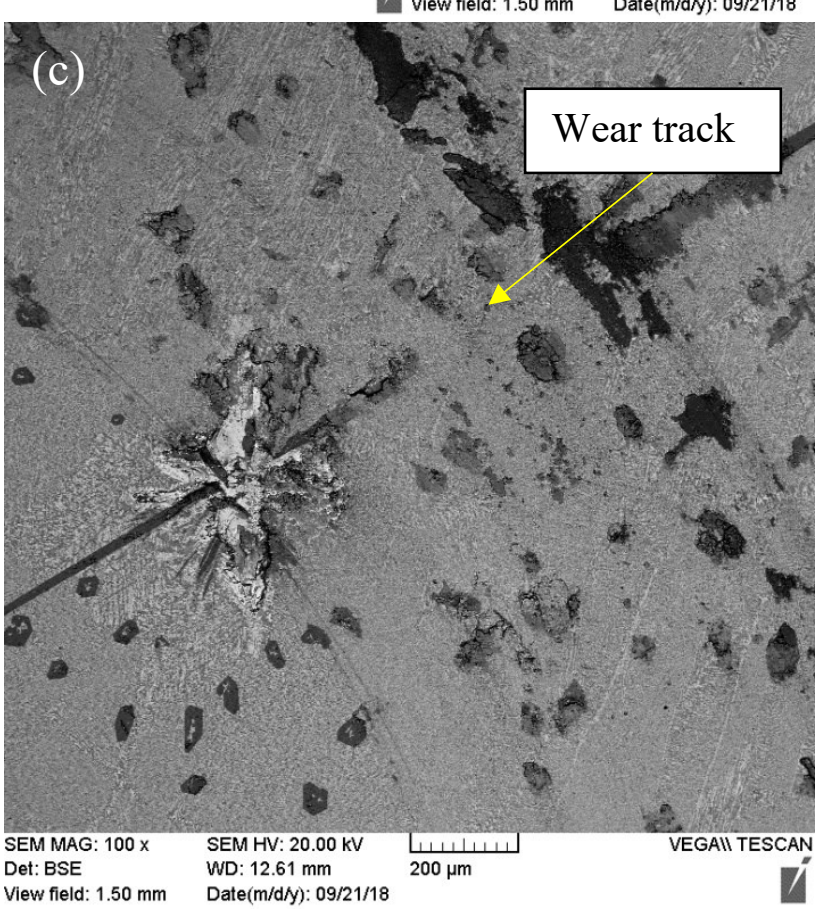

Figure 3-9: SEM images of worn surface (a) Stellite 706, (b) Stellite 712 and (c) Stellite 720 


\section{Chapter: Results and Discussion}

At least three tests were performed on each alloy under the same conditions to repeat the measurements and the results showed high repeatability. Thus, one of the curves from the multiple tests for each condition is presented in this chapter.

\subsection{Open Circuit Potential}

OCP test is initially done as a standard in electrochemical testing in order to accomplish two important tasks. It is primarily used to stabilize the electrochemical system in order to allow the alloy immersed in a certain electrolytic solution to reach close to equilibrium and to determine the starting point of cyclic polarization. From all 1.5 hours OCP testing done on each alloy, the stable negative potential value reached ranged between $0.25-0.35 \mathrm{~V}$, hence the starting point for all cyclic polarization tests are set to $-0.5 \mathrm{~V}$. The timing chosen for the OCP duration would usually reflect on how stable the system will eventually become, however, this may not always be true for all alloys. The OCP duration is established by trial and error where depending on the alloy tested and the electrolytic solution used, it can take as little as 20 minutes or up to 168 hours for alloys to reach stability. In the case of Stellite 700 series alloys tested, which have corrosion protective elements such as $\mathrm{Cr}$ and $\mathrm{Mo}$, the expectation is that higher OCP durations would allow the alloys to reach further stability. If further stability is reached where the protective passive film on the alloy surface grows or becomes more stable, this will be reflected on the quantitative results in EIS and cyclic polarization. The OCP is measured as a function of time and the results are displayed in Figure 4-1 to Figure 4-4 for all tested Stellite alloys for both surface and temperature conditions for 1.5 hours in morpholine. 


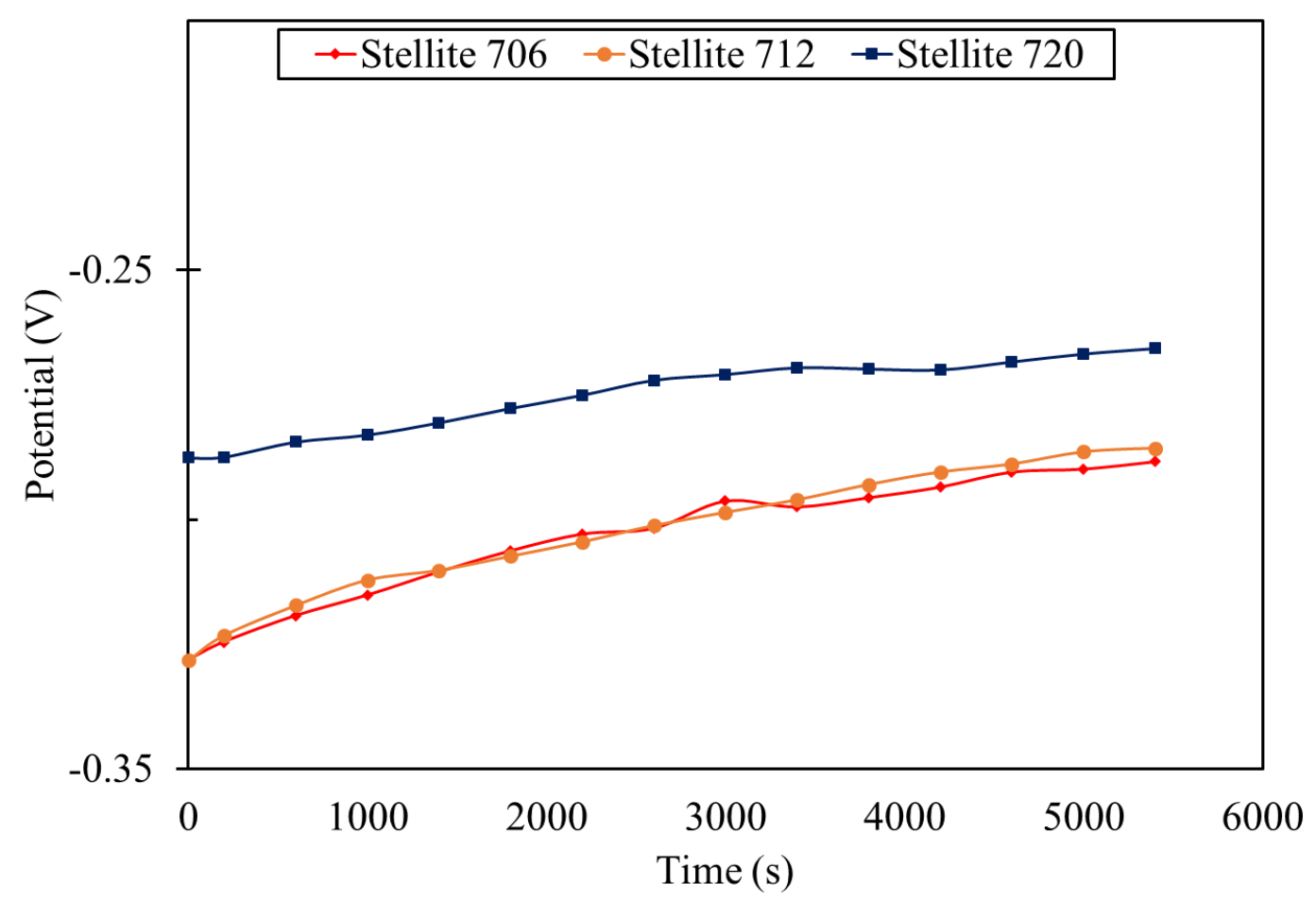

Figure 4-1: $\mathrm{OCP}$ curves at $25^{\circ} \mathrm{C}$

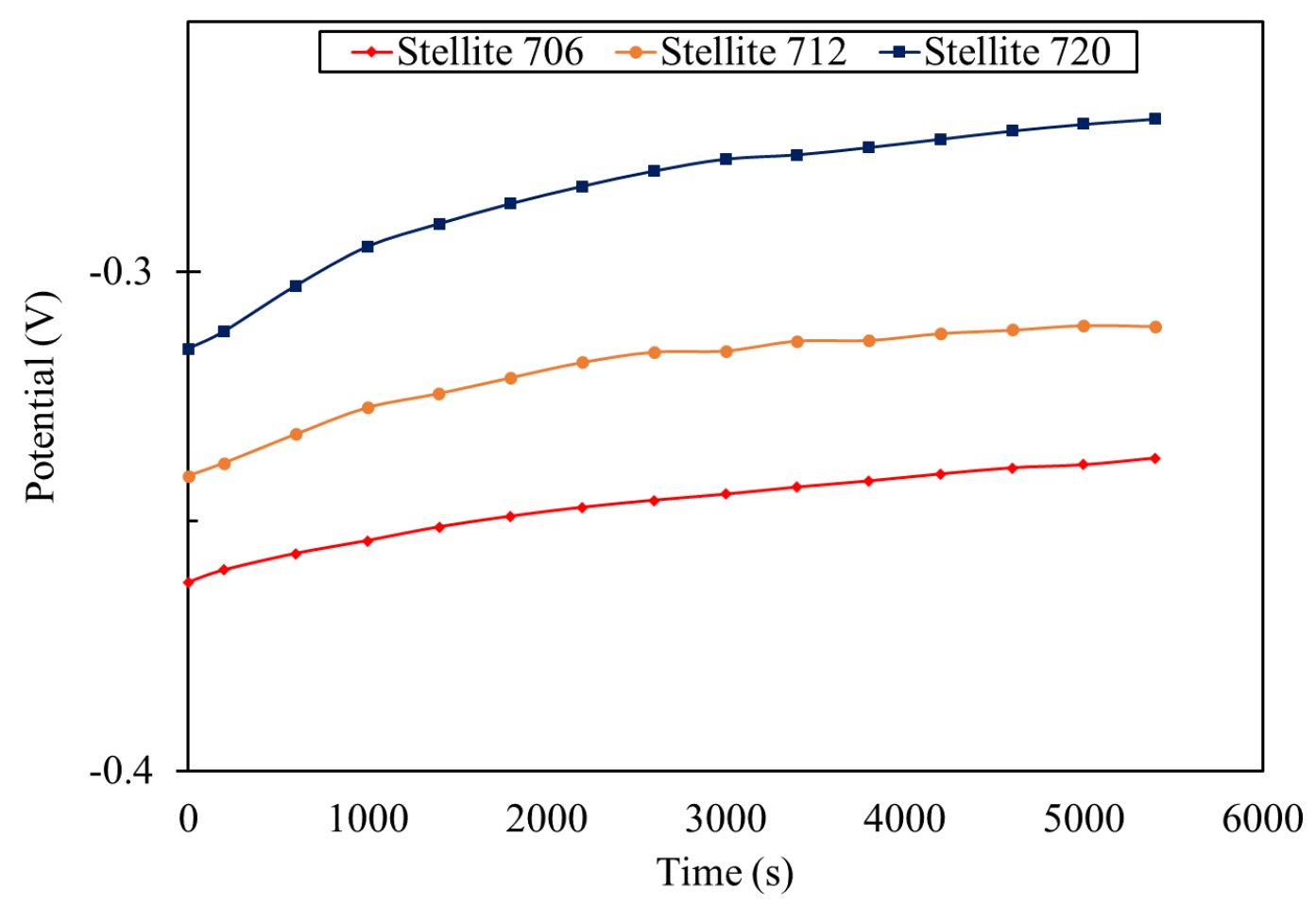

Figure 4-2: $\mathrm{OCP}$ curves at $50^{\circ} \mathrm{C}$ 


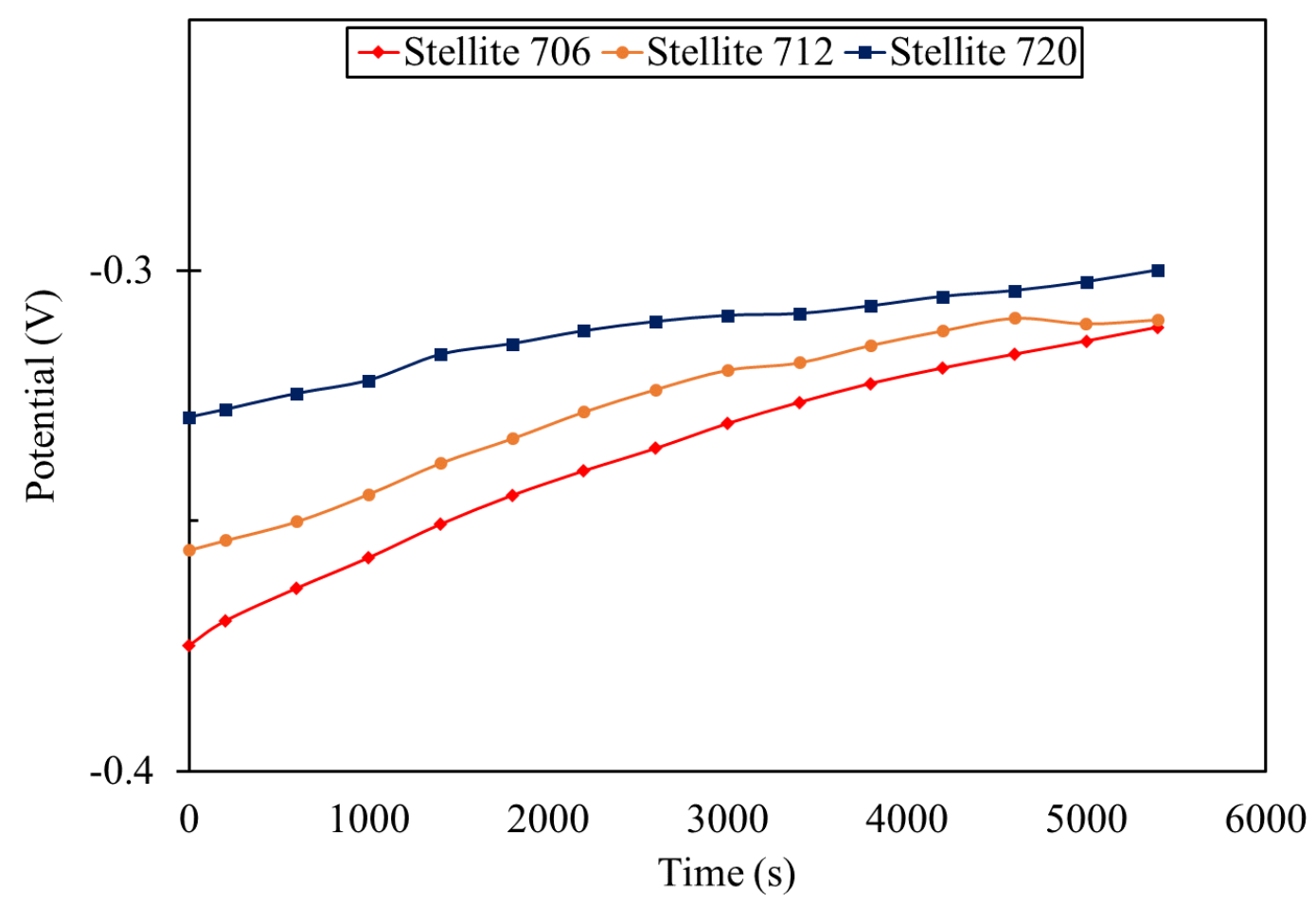

Figure 4-3: OCP curves of worn alloys at $25^{\circ} \mathrm{C}$

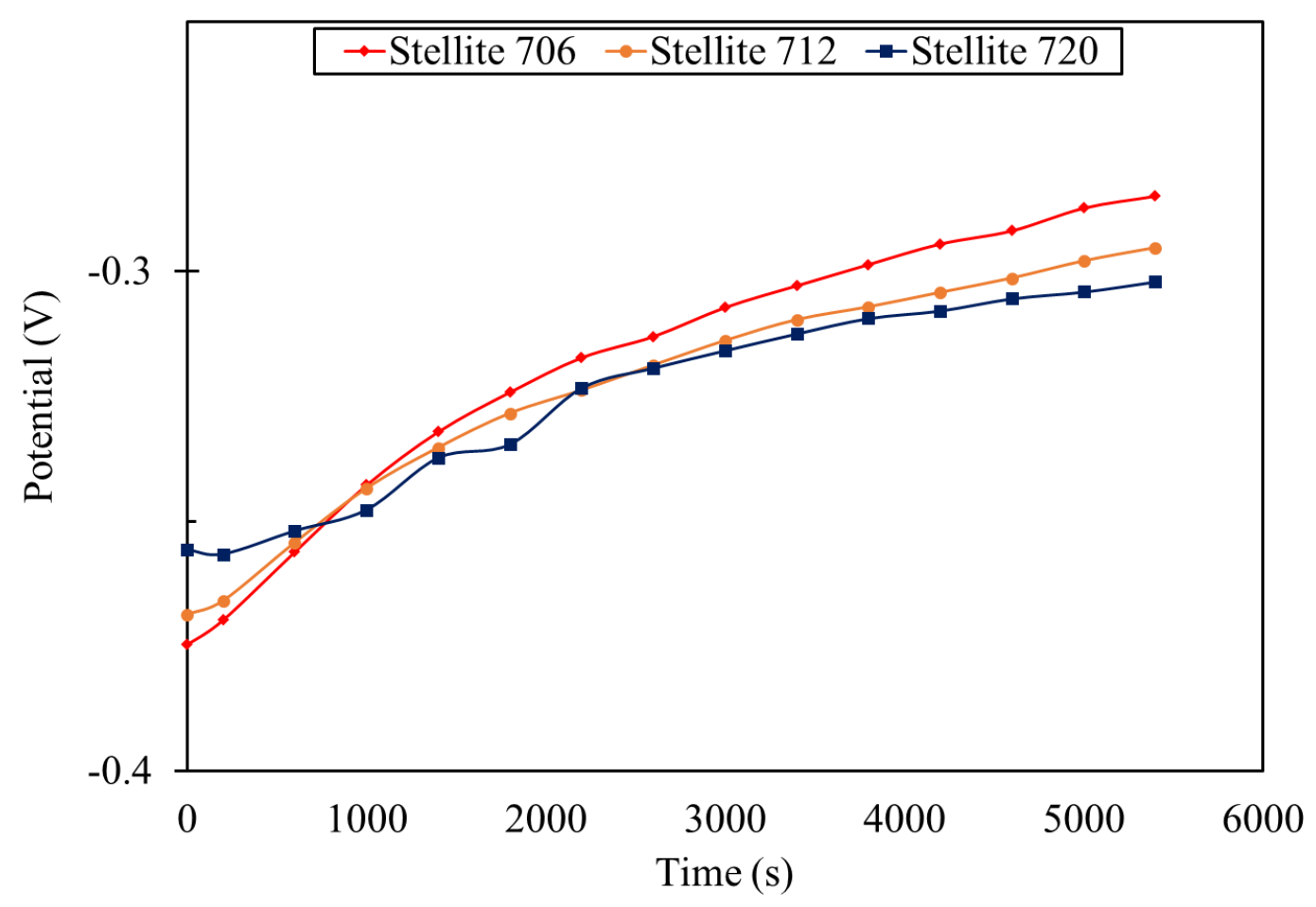

Figure 4-4: OCP curves of worn alloys at $50^{\circ} \mathrm{C}$ 
The final OCP value at the end of the test signifies the stability the alloy reached within the time set. The variation in final OCP value among the Stellite alloys did not show any significant differences. However, qualitatively since Stellite 720 displayed a slightly more positive value in comparison, it should have a slightly better performance in both EIS and cyclic polarization. OCP is also conducted for 24 and 48 hours at $25^{\circ} \mathrm{C}$ only and the results are displayed in Figure 4-5 and Figure 4-6. As mentioned earlier in Section 3.3, the sample holder has a limitation where it will effectively be damaged if it is held at $50^{\circ} \mathrm{C}$ and beyond for a long period of time. Due to this limitation, higher OCP time tests are not done at $50^{\circ} \mathrm{C}$ for both EIS and polarization.

With higher OCP duration it is observed that the final value ranged between $-0.25 \mathrm{~V}$ and $-0.3 \mathrm{~V}$ for 24 hours, whereas the range goes up slight between $-0.2 \mathrm{~V}$ and $-0.25 \mathrm{~V}$ for 48 hours. This is a clear indication that the Stellite alloys with greater immersion time in morpholine eventually displayed better stability where their potential value slightly becomes more positive. Once again, the reflection of such stability is given in the quantitative values for both EIS and polarization in Section 4.2 and Section 4.3. 


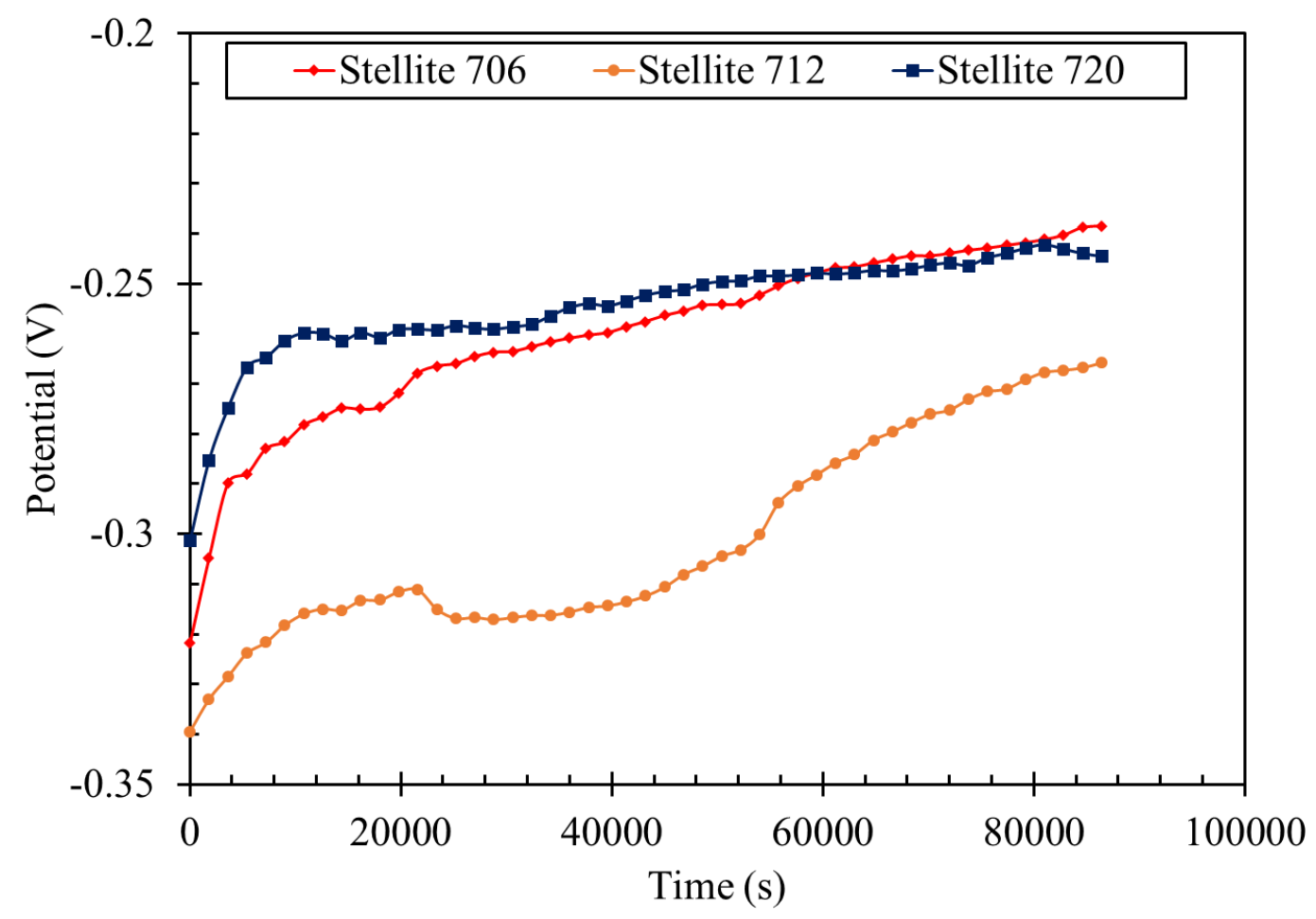

Figure 4-5: $24 \mathrm{hr}$ OCP curves of intact alloys at $25^{\circ} \mathrm{C}$

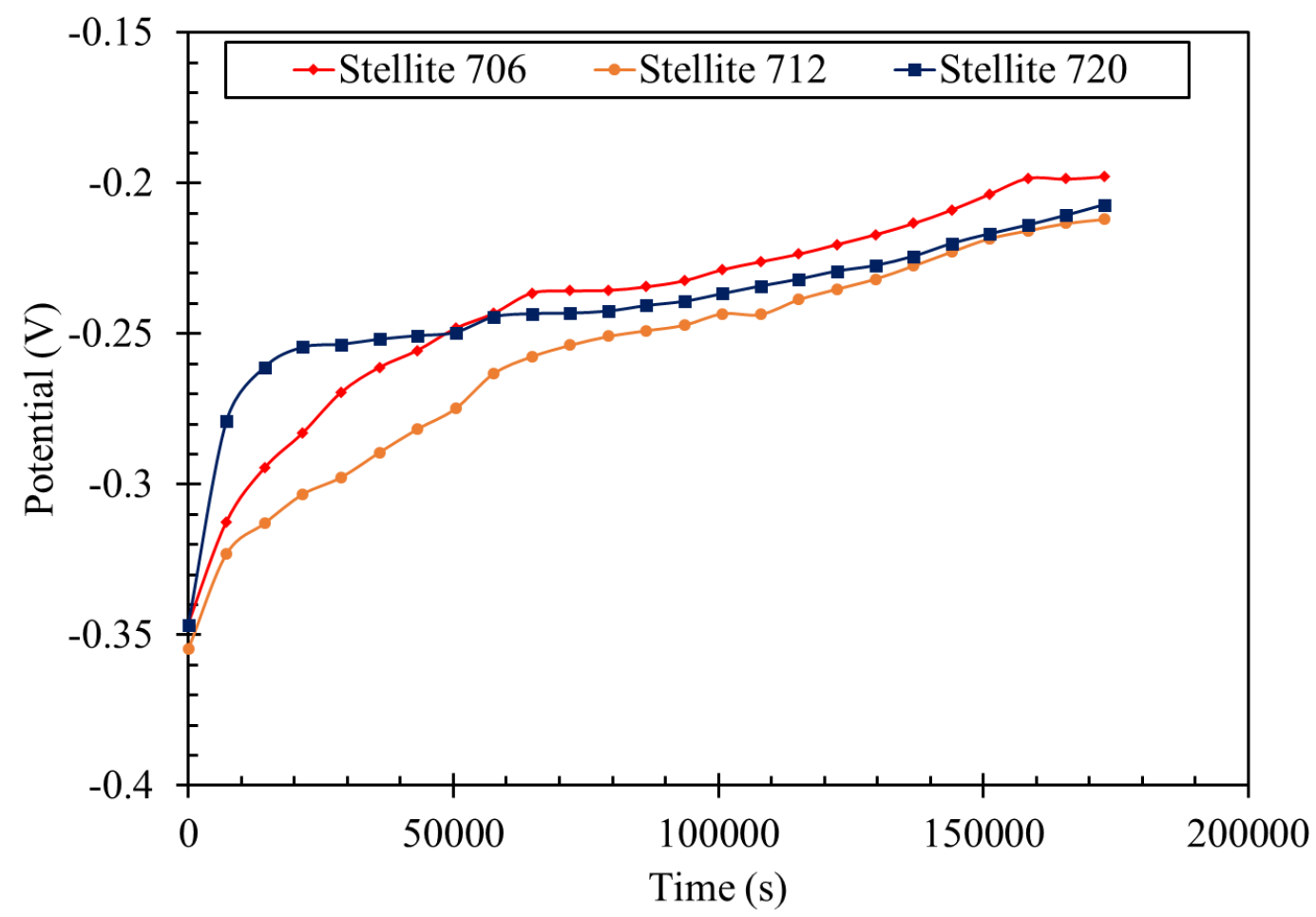

Figure 4-6: $48 \mathrm{hr}$ OCP curves of intact alloys at $25^{\circ} \mathrm{C}$ 


\subsection{EIS Results}

\subsubsection{Bode plots}

Corrosion is about the flow of electrons from a corrosive medium to a metal surface. If the flow is stopped, the corrosion stops. Thus, having an oxide film layer is an effective way to prevent further degradation of metallic alloys over time. As discussed earlier, Stellite alloys have Cr as a major alloying element, thus when exposed to oxygen/acid medium, they form a protective Cr-rich oxide layer $\left(\mathrm{Cr}_{2} \mathrm{O}_{3}\right.$ or $\left.\mathrm{CrO}_{4}{ }^{2-}\right)$, depending on the potential applied [38]. This layer acts as a chemical barrier on the metal substrate and prevents extraterrestrial chemical species from passing through. One of the main desirable aspects of Cr-rich oxide is its ability to self-passivate and prevent further corrosion over time. It is already well known that $\mathrm{Cr}$ does react with most acids, but it is unknown how it will behave in a basic solution such as morpholine at a certain $\mathrm{pH}$ value of 9.5.

If the oxide layer is stable within the environment a Stellite alloy is exposed to, EIS can be used to quantify the performance of the oxide layer. Figure 4-7 shows an example of Stellite 706 bode plot at 1.5, 24 and 48 hours of OCP. The other two alloys have the same bode plot trend as Stellite 706. 


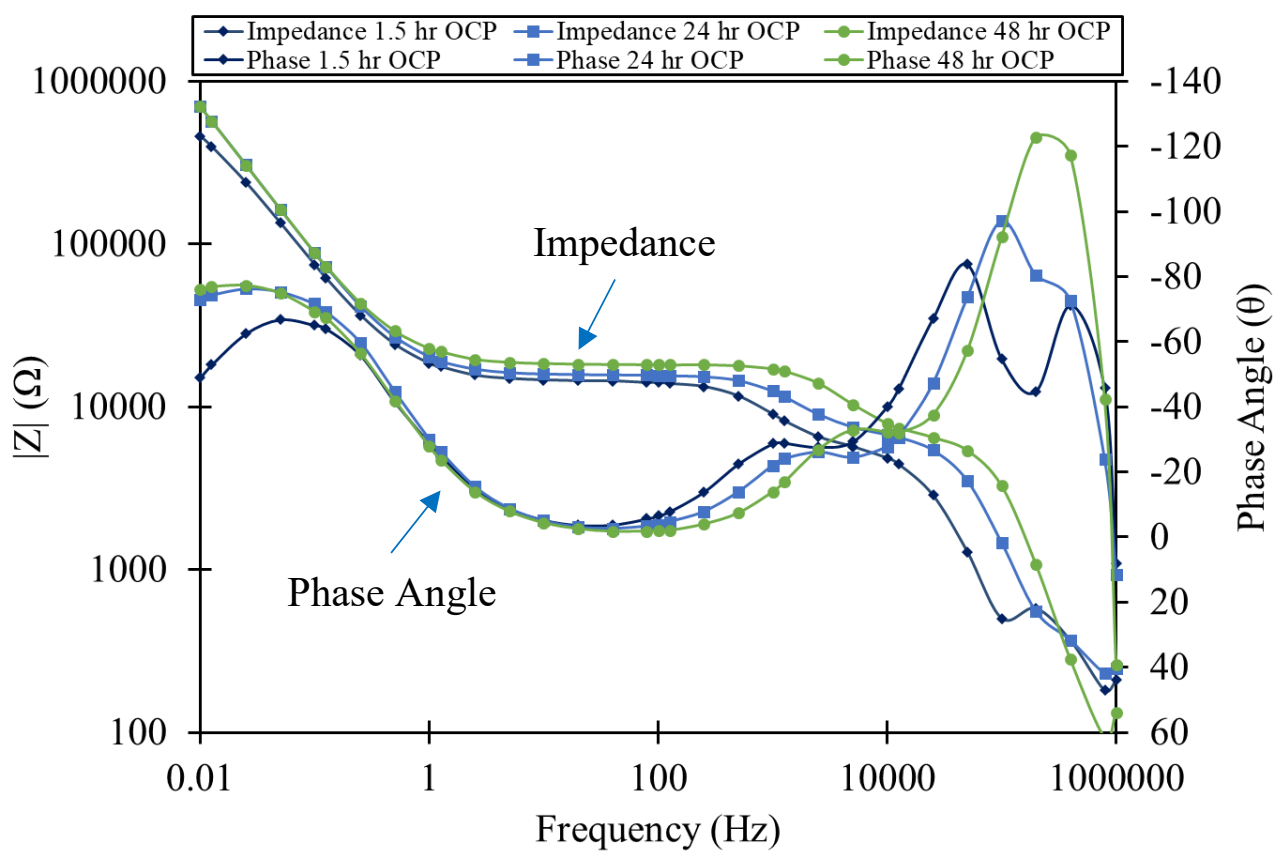

Figure 4-7: Stellite 706 bode plot for different OCP durations

As demonstrated in Figure 4-7, the high-frequency region from $10^{4}-10^{6} \mathrm{~Hz}$ is extremely distorted and cannot be interpreted with accuracy. This distortion can be caused by many factors such as the machine itself, the electrolyte solution, the coaxial cables, or salt bridge. This region has changes in phase angle going from capacitance to inductance and vise-versa. It gives no meaningful or relevant data points to help analyze the oxide behaviour of the Stellite alloys. Since the highfrequency range is mostly distorted, it cannot give a good fitting when modelling the equivalent circuit model. However, it is entirely possible that the middle frequency horizontal line is the solution resistance. To test this hypothesis, solution resistance test was conducted on morpholine alone to investigate if there was a difference in impedance behaviour in mid to high-frequency ranges. Morpholine solution impedance was measured and compared with Stellite 706 impedance for different OCP times, as shown in Figure 4-8. 


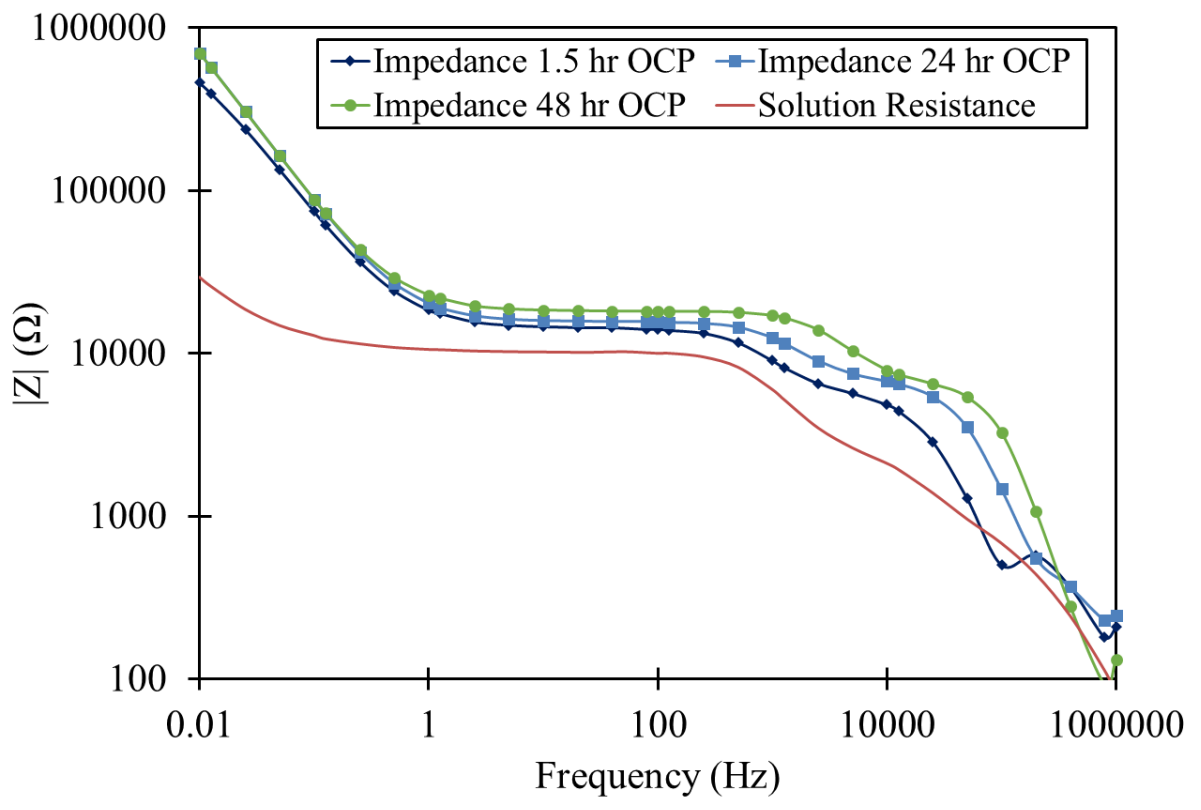

Figure 4-8: Morpholine solution resistance and Stellite 706 impedance curves for different OCP durations

The result showed that the solution resistance curve of morpholine has the same trend as the alloy curves. The horizontal portion of its resistance curve, which is approximately $10 \mathrm{k} \Omega$ is very close to the horizontal resistance values of the actual alloy. An alloy would have a higher impedance value and hence the horizontal portion of the impedance curve is a bit higher. To further verify if the horizontal portion of the curve is the solution resistance, a similar solution resistance measurement was made using $3.5 \mathrm{wt} . \% \mathrm{NaCl}$, deionized water and tap water. The comparison of the four solutions is shown in Figure 4-9. 


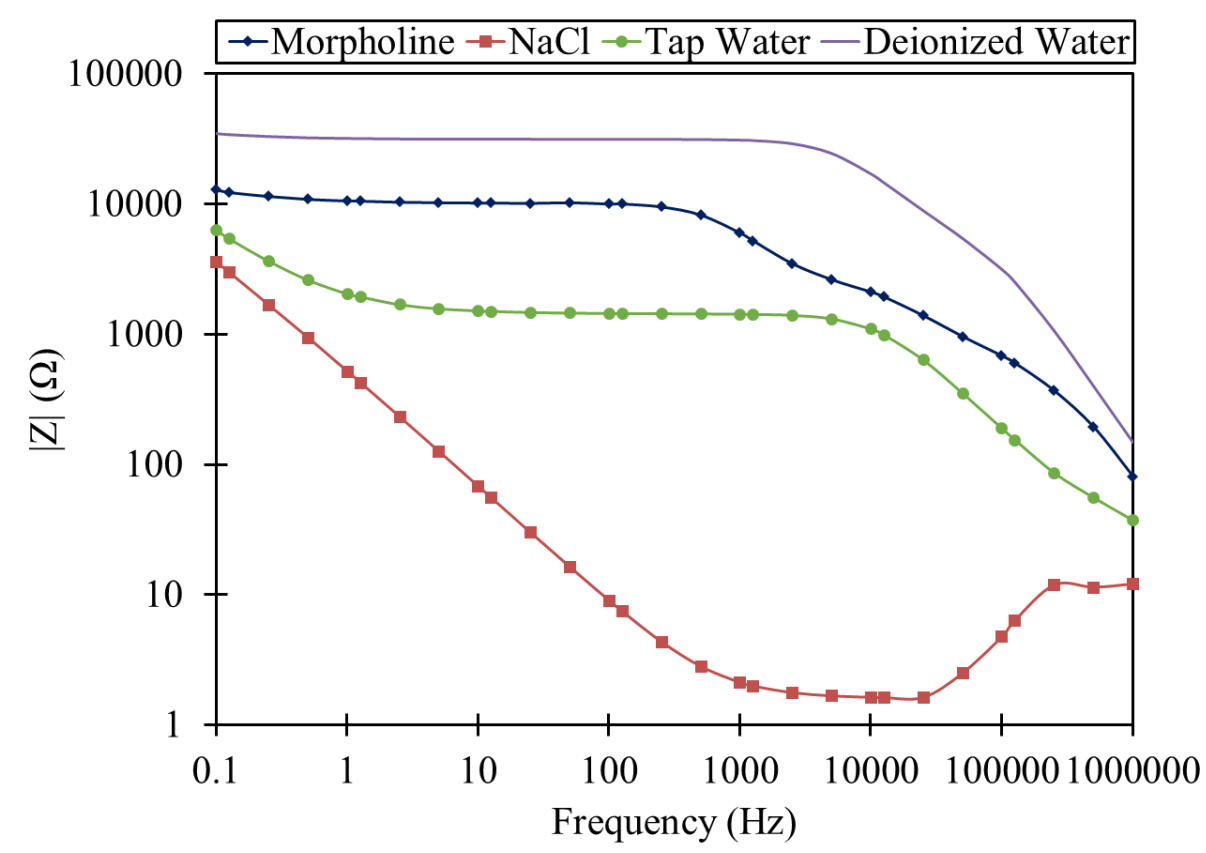

Figure 4-9: Solution resistance comparison

As expected, deionized water has the highest impedance given that there are little to no ions for conductivity. The second highest is morpholine, the third is tap water and lastly the $\mathrm{NaCl}$, which would be the most conductive out of all. Once again, the distortion in the higher frequency range is observed. To determine what had caused the distortion, some of the testing instrument components are investigated. Different co-axial cables were used to see if the cable caused the distortion, but it did not change the resulting impedance curve behaviour. However, once the additional salt bridge was removed from the test cell, where the RE alone was placed in the electrolyte, a change in the curves was observed, as demonstrated in Figure 4-10. Tap water and deionized water are shown as examples where the high-frequency range shifted towards the right by one order of magnitude when the salt bridge was not used, and the distortion was much less. 
Morpholine and $\mathrm{NaCl}$ behaved similarly as the curves shifted towards the right when the salt bridge was removed.

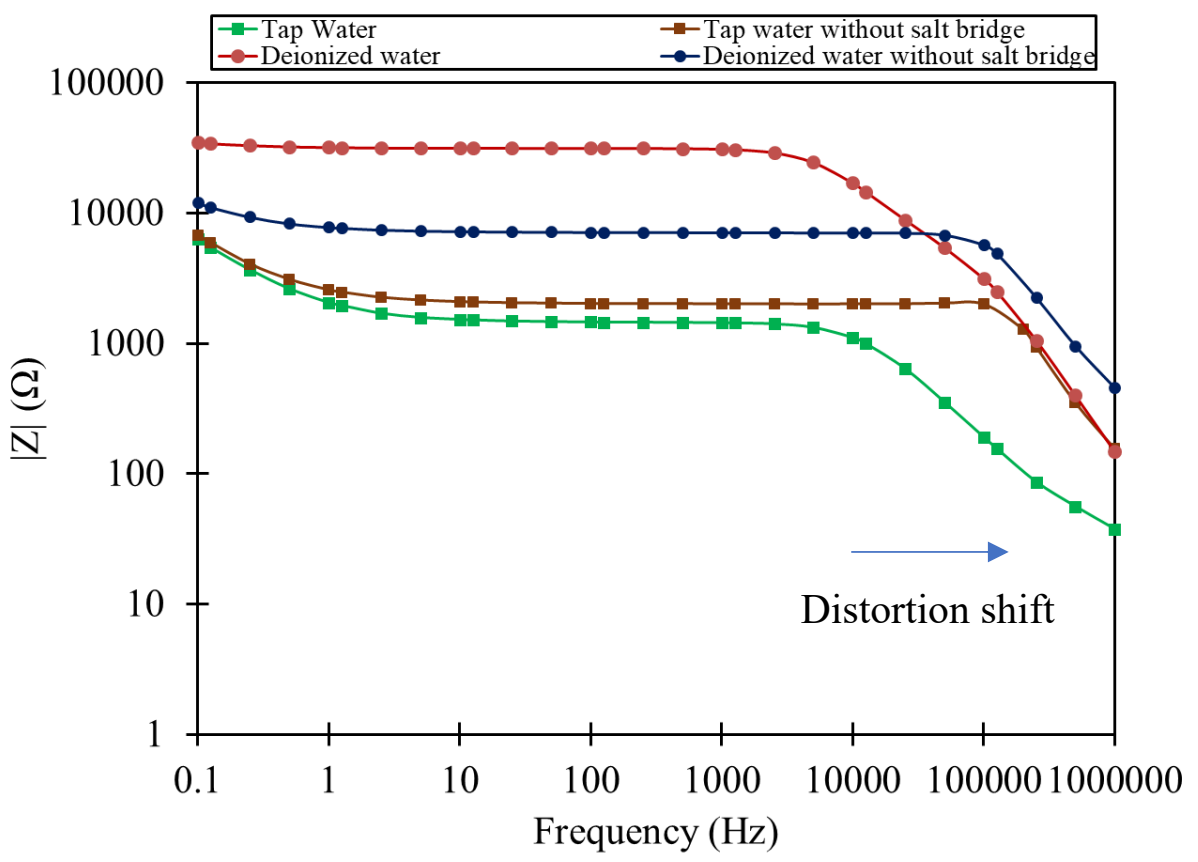

Figure 4-10: Impedance plots showing the effect of salt bridge not used

Given that the salt bridge induced the distortion in the high-frequency range, it would make sense to conduct all following EIS tests with the salt bridge removed. However, the measurement without the salt bridge can introduce other problems such as $\mathrm{KCl}$ solution leaking into the electrolyte from the RE. The main purpose of using the salt bridge is to minimize saturated $\mathrm{KCl}$ leaking into the main electrolyte solution. If the leaks were to occur, the EIS and polarization test results would possibly be compromised, because introducing new ions into the electrolyte solution would change the electrochemical reactions in the system. Thus, to make use of the EIS data obtained, all EIS results presented have the frequency set to $10^{3} \mathrm{~Hz}$ maximum, with all data in the high frequency 
removed. The horizontal impedance portion can then be correctly treated as solution resistance, as observed from the standalone solution resistance test in Figure 4-9.

Figure 4-11 and Figure 4-12 show bode plot of the three intact surface Stellite alloys at $25^{\circ} \mathrm{C}$ and $50^{\circ} \mathrm{C}$. The trends of impedance and phase angle are similar for both temperature and surface conditions. The higher temperature from a visual perspective did not introduce any changes to the alloy surface after 1.5 hours of OCP. The trends are clear at the lower frequencies with the negative slope indicating a pseudo-capacitive behaviour of the oxide layer and the horizontal portion at higher frequencies indicating a resistive behaviour, which is the solution resistance. The capacitive and resistive behaviour is also correlated with the phase angle behaviour across those frequency ranges, where capacitive behaviour has the phase angle moving towards $-90^{\circ}$ and horizontal solution resistance being near $0^{\circ}$. The high-frequency range has a lot of distortion with a mixture of capacitive and inductive behaviour and as such has been removed from the bode plots as mentioned earlier. 


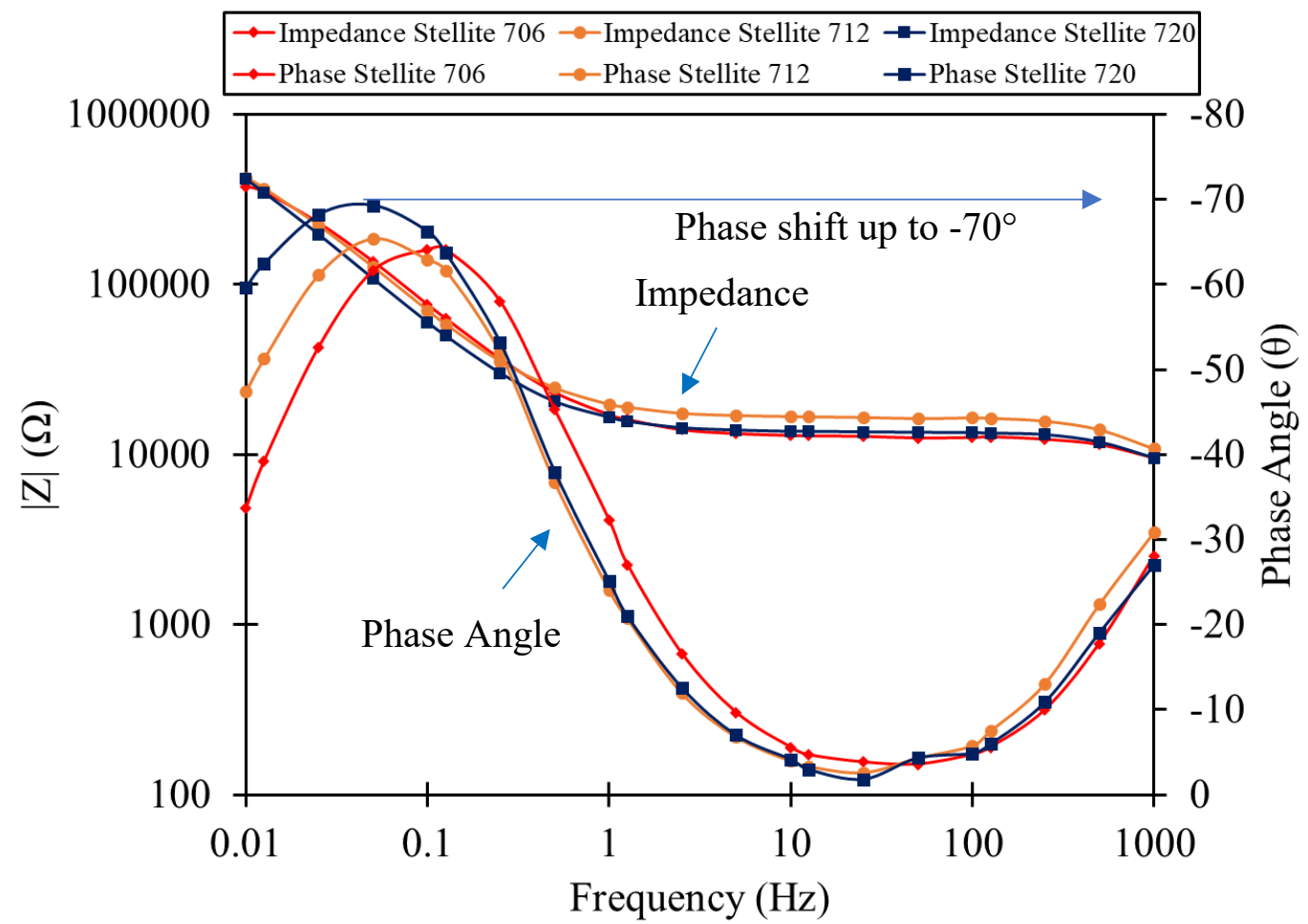

Figure 4-11: Bode plot for intact specimens at $25^{\circ} \mathrm{C}$

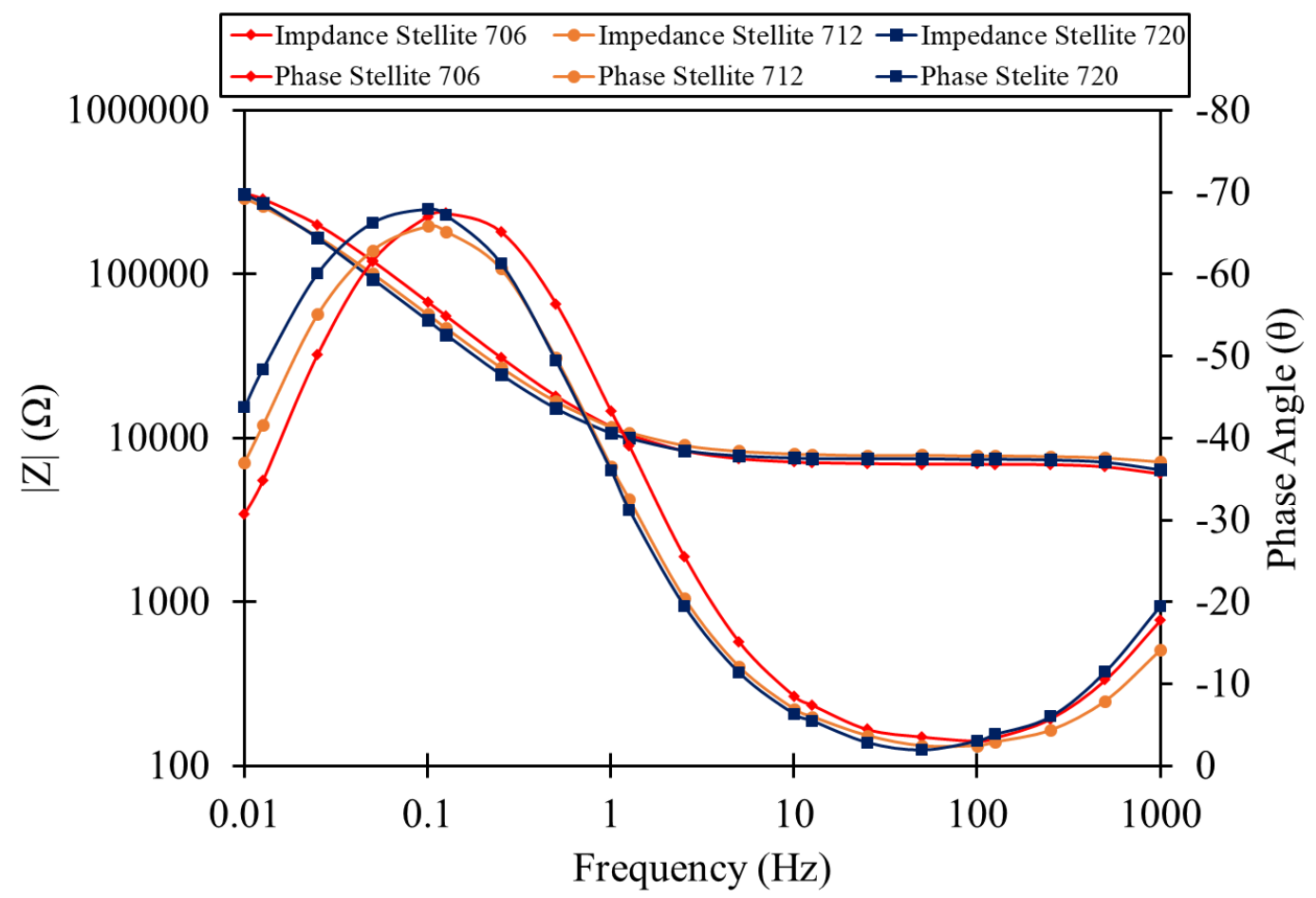

Figure 4-12: Bode plot for intact specimens at $50^{\circ} \mathrm{C}$ 


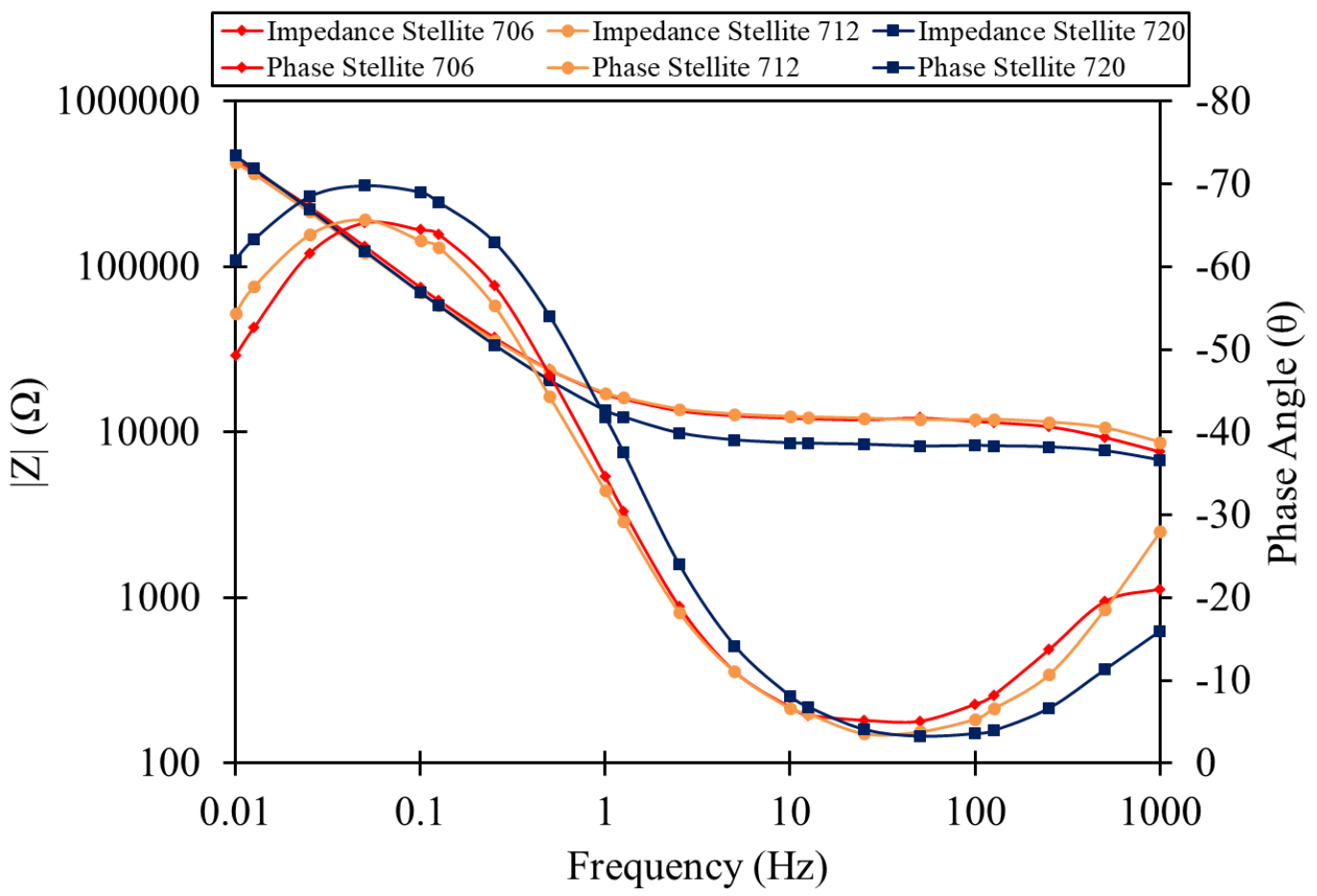

Figure 4-13: Bode plot for worn specimens at $25^{\circ} \mathrm{C}$

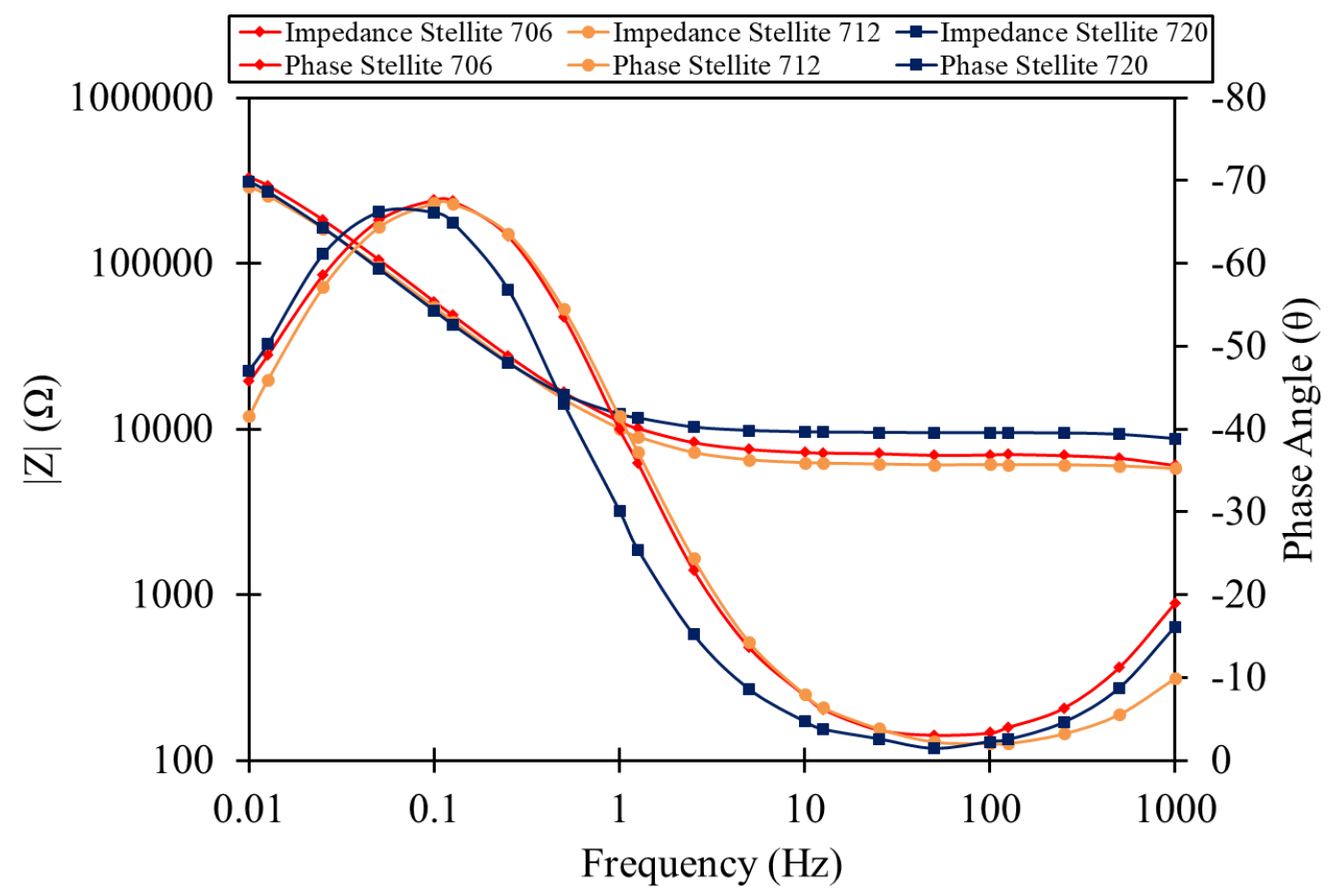

Figure 4-14: Bode plot for worn specimens at $50^{\circ} \mathrm{C}$ 
Qualitatively the bode plots display no significant difference among the alloys for both temperature and surface conditions, however, the changes can be recognized by reviewing the quantitative values summarized in Table 4-1. These numerical values obtained had chi-squared $\left(\chi^{2}\right)$ error values less than $10^{-3}$ and the errors associated with each individual element are up to $7 \%$. The two important parameters are the solution resistance $\left(R_{s}\right)$ and parallel resistance $\left(R_{p}\right)$ values. The $R_{p}$ values indicate the oxide layer integrity and the $R_{S}$ values indicate the resistance of the electrolytic solution to charge transfer. With the $n$ value for CPE being close to 1 , a very good capacitive behaviour of the oxide film in resisting charge transfer is indicated. The $R_{S}$ values for each temperature are relatively close to one another with very high resistance values ranging from 10 $18 \mathrm{k} \Omega$ for $25^{\circ} \mathrm{C}$ and $7-9 \mathrm{k} \Omega$ for $50^{\circ} \mathrm{C}$. Figure $4-15$ and Figure $4-16$ is a simpler representation of Table 4-1 showing the $R_{p}$ comparison amongst the alloys.

Table 4-1: EIS results for the tests after $1.5 \mathrm{hr}$ OCP

\begin{tabular}{|c|c|c|c|c|c|}
\hline Temp & Alloy & $\mathbf{1 0}^{\mathbf{6}} \times \mathbf{C P E}\left(\mathbf{\Omega}^{-\mathbf{1}} \mathbf{s}^{\mathbf{n}}\right)$ & $\mathbf{n}$ & $\mathbf{R}_{\mathbf{p}} \mathbf{( k \Omega} \mathbf{)}$ & $\mathbf{R}_{\mathbf{s}}(\mathbf{k} \mathbf{\Omega})$ \\
\hline \multirow{3}{*}{$25^{\circ} \mathrm{C}$} & Stellite 706 & $20.8 \pm 0.1$ & $0.91 \pm 0.004$ & $689 \pm 23$ & $13.6 \pm 0.1$ \\
\cline { 2 - 6 } & Stellite 712 & $23.4 \pm 0.2$ & $0.91 \pm 0.005$ & $663 \pm 23$ & $18.0 \pm 0.1$ \\
\cline { 2 - 6 } & Stellite 720 & $26.8 \pm 0.2$ & $0.91 \pm 0.003$ & $999 \pm 39$ & $14.2 \pm 0.1$ \\
\hline \multirow{3}{*}{$50^{\circ} \mathrm{C}$} & Stellite 706 & $21.5 \pm 0.1$ & $0.90 \pm 0.003$ & $626 \pm 18$ & $9.3 \pm 0.1$ \\
\cline { 2 - 6 } & Stellite 712 & $25.8 \pm 0.1$ & $0.90 \pm 0.002$ & $402 \pm 5$ & $7.3 \pm 0.02$ \\
\cline { 2 - 6 } & Stellite 720 & $26.4 \pm 0.2$ & $0.90 \pm 0.004$ & $521 \pm 13$ & $9.1 \pm 0.1$ \\
\hline \multirow{3}{*}{$25^{\circ} \mathrm{C}$} & Worn Stellite 706 & $18.6 \pm 0.1$ & $0.87 \pm 0.004$ & $1055 \pm 45$ & $12.6 \pm 0.1$ \\
\cline { 2 - 6 } & Worn Stellite 712 & $21.0 \pm 0.1$ & $0.86 \pm 0.003$ & $1135 \pm 34$ & $9.7 \pm 0.05$ \\
\cline { 2 - 6 } & Worn Stellite 720 & $22.1 \pm 0.1$ & $0.87 \pm 0.003$ & $1433 \pm 57$ & $10.6 \pm 0.05$ \\
\hline \multirow{3}{*}{$50^{\circ} \mathrm{C}$} & Worn Stellite 706 & $25.6 \pm 0.1$ & $0.90 \pm 0.002$ & $544 \pm 8$ & $7.9 \pm 0.03$ \\
\cline { 2 - 6 } & Worn Stellite 712 & $29.0 \pm 0.1$ & $0.89 \pm 0.001$ & $455 \pm 4$ & $5.7 \pm 0.01$ \\
\cline { 2 - 6 } & Worn Stellite 720 & $29.2 \pm 0.1$ & $0.90 \pm 0.002$ & $637 \pm 9$ & $7.7 \pm 0.02$ \\
\hline
\end{tabular}




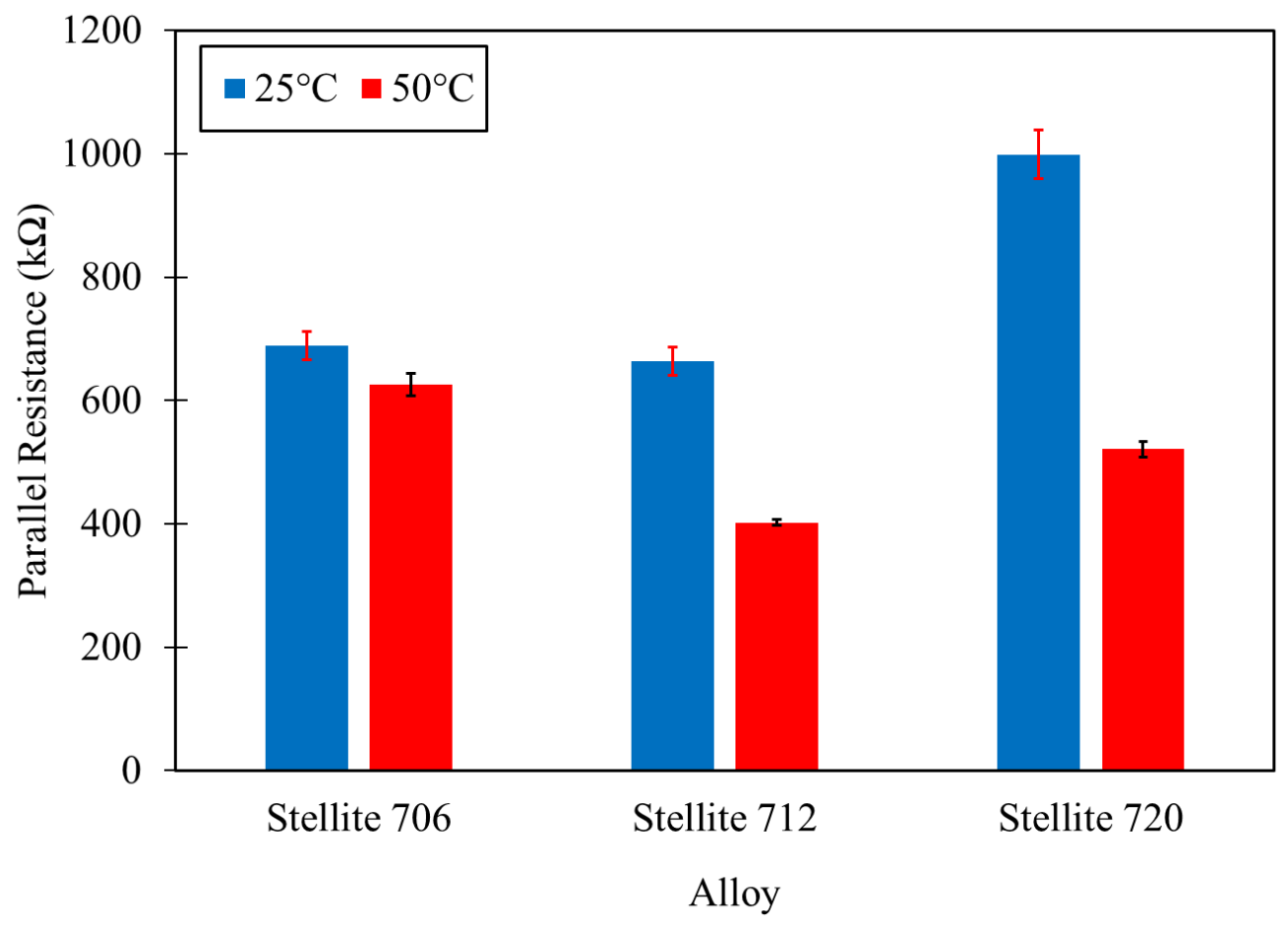

Figure 4-15: Bar graph representation of $R_{p}$ for intact specimens

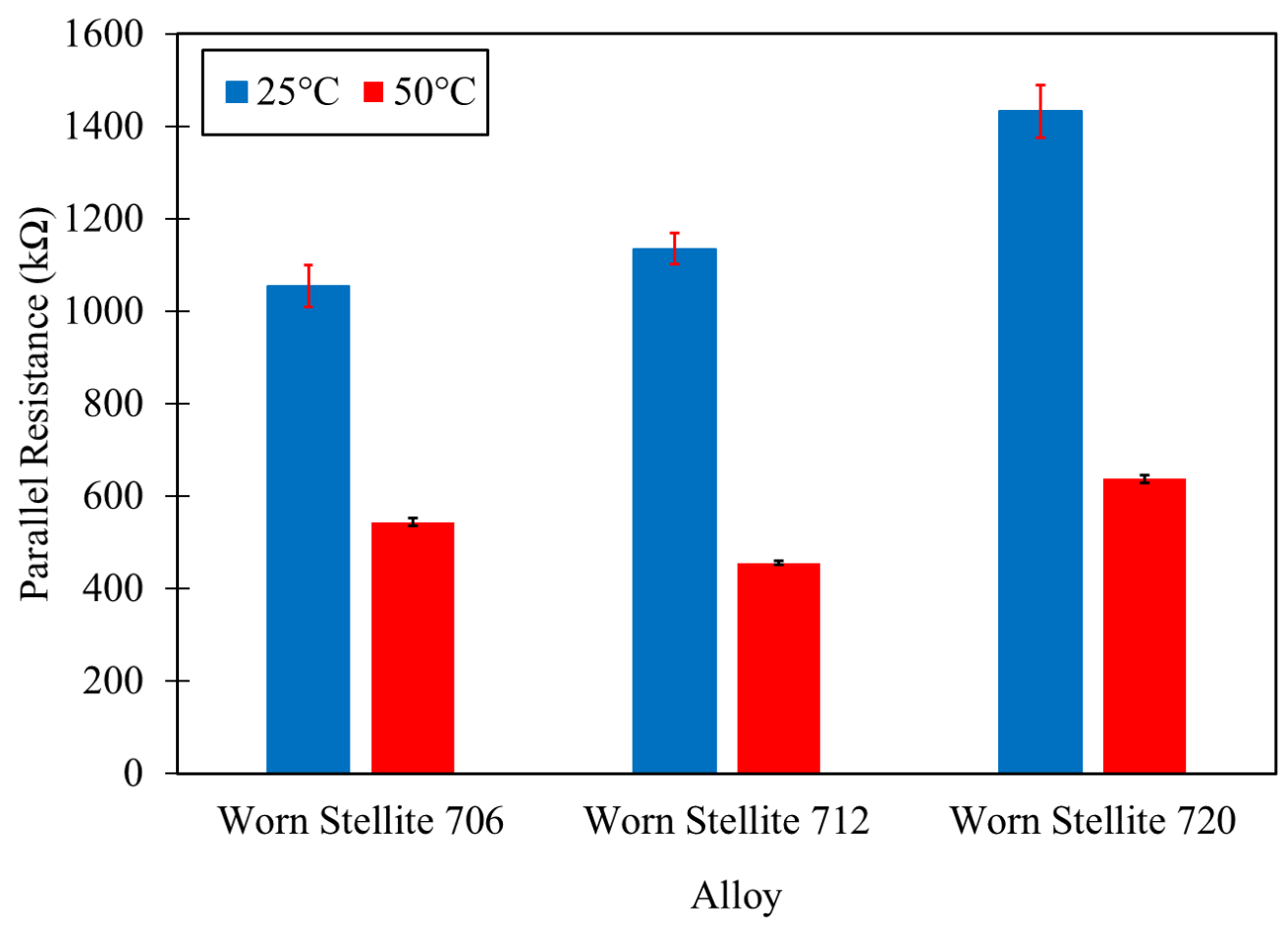

Figure 4-16: Bar graph representation of $\boldsymbol{R}_{p}$ for worn specimens 
From the bar graphs, Stellite 720 displays a better performance in comparison for both temperature and surface conditions apart from intact Stellite 706, which had a slightly better performance at $50^{\circ} \mathrm{C}$. The elevated temperature does influence $R_{p}$ for all the alloys as it reduces the oxide resistance performance. For the higher OCP durations, qualitatively the bode plot trends look similar in Figure 4-17 and Figure 4-18 compared to the figures for 1.5 hours OCP. However, quantitatively the $R_{p}$ value increased dramatically with Stellite 720 having the better performance in comparison shown in Figure 4-19 and Table 4-2. Figure 4-19 shows a simplified representation of $R_{p}$ increase with higher OCP duration. This relationship is correlated with the Nyquist plot result in Figure 4-22 for 24 and 48 hours OCP where higher $R_{p}$ values are represented as larger semicircles. The results clearly show that $R_{p}$ increases with higher OCP duration where the Cr oxide protective properties are greatly improved with higher Mo content. The $R_{S}$ values remain consistent within the range $11-18 \mathrm{k} \Omega$ for each OCP time. The $R_{p}$ values obtained also satisfy the R-CPE circuit model mathematically where from Eq (13) the $R_{p}$ values from the table represent impedance $\mathrm{Z}_{C P E}$, thus if $\mathrm{Z}_{C P E}$ is larger, the $Q$ capacitance $(\mathrm{CPE})$ will be smaller. As the oxide film becomes increasingly stable, which is depicted by the larger $R_{p}$ impedance value, the fitting parameter $n$ will also slightly increase signifying the pseudo-capacitive behaviour gets closer to $-90^{\circ}$ phase shift as seen in Figure 4-17 and Figure 4-18. 


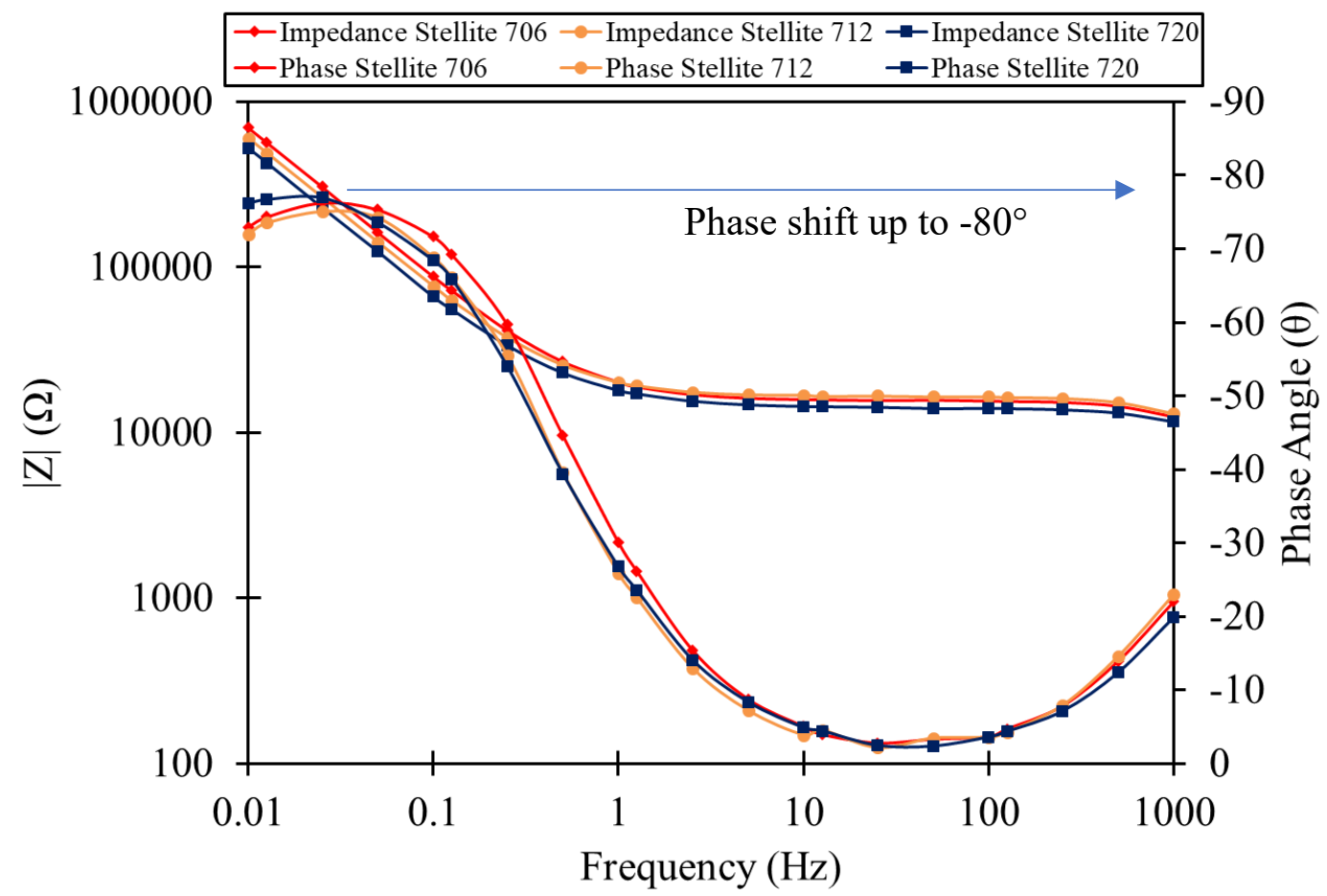

Figure 4-17: $24 \mathrm{hr}$ OCP bode plot for intact specimens at $25^{\circ} \mathrm{C}$

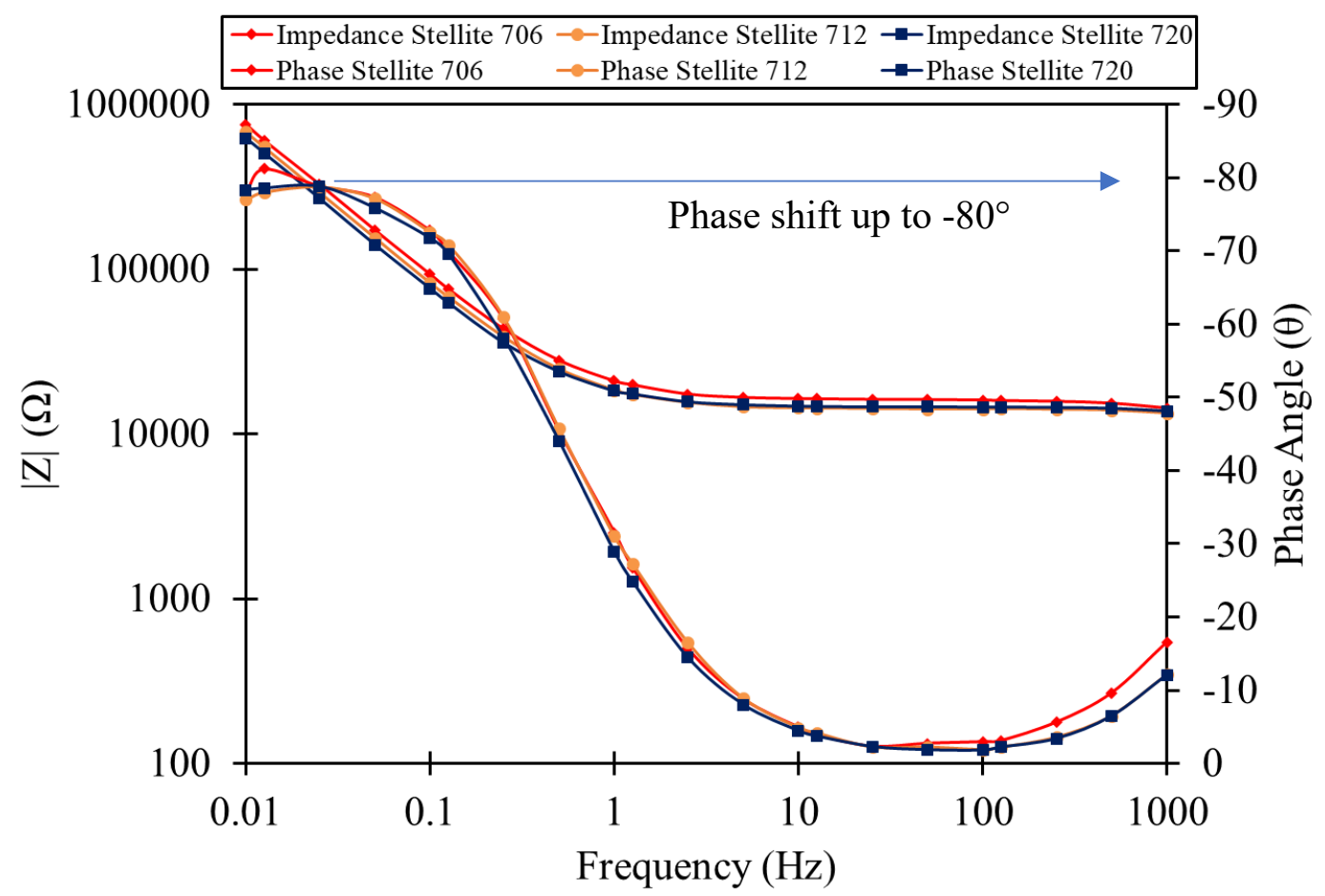

Figure 4-18: $48 \mathrm{hr}$ OCP bode plot for intact specimens at $25^{\circ} \mathrm{C}$ 
Table 4-2: Comparison of EIS results for different $\mathrm{OCP}$ durations at $25^{\circ} \mathrm{C}$

\begin{tabular}{|c|c|c|c|c|c|}
\hline Alloy & Time (hr) & $\mathbf{1 0}^{\mathbf{6}} \times \mathbf{C P E}\left(\mathbf{\Omega}^{\mathbf{- 1}} \mathbf{s}^{\mathbf{n}}\right)$ & $\mathbf{n}$ & $\mathbf{R}_{\mathbf{p}} \mathbf{( k \boldsymbol { } \mathbf { } )}$ & $\mathbf{R}_{\mathbf{s}}(\mathbf{k} \mathbf{\Omega})$ \\
\hline \multirow{3}{*}{ Stellite 706 } & 1.5 & $20.8 \pm 0.1$ & $0.91 \pm 0.004$ & $689 \pm 23$ & $13.6 \pm 0.1$ \\
\cline { 2 - 6 } & 24 & $16.2 \pm 0.1$ & $0.91 \pm 0.001$ & $6579 \pm 156$ & $13.6 \pm 0.06$ \\
\cline { 2 - 6 } & 48 & $15.7 \pm 0.1$ & $0.92 \pm 0.001$ & $9226 \pm 403$ & $14.7 \pm 0.05$ \\
\hline \multirow{3}{*}{ Stellite 712 } & 1.5 & $23.4 \pm 0.2$ & $0.91 \pm 0.005$ & $663 \pm 23$ & $18.0 \pm 0.1$ \\
\cline { 2 - 6 } & 24 & $18.7 \pm 0.05$ & $0.91 \pm 0.002$ & $5405 \pm 305$ & $12.7 \pm 0.04$ \\
\cline { 2 - 6 } & 48 & $17.7 \pm 0.1$ & $0.92 \pm 0.001$ & $8807 \pm 417$ & $11.1 \pm 0.04$ \\
\hline \multirow{3}{*}{ Stellite 720 } & 1.5 & $26.8 \pm 0.2$ & $0.91 \pm 0.003$ & $999 \pm 39$ & $14.2 \pm 0.1$ \\
\cline { 2 - 6 } & 24 & $20.9 \pm 0.1$ & $0.92 \pm 0.002$ & $6992 \pm 480$ & $11.8 \pm 0.06$ \\
\cline { 2 - 6 } & 48 & $19.3 \pm 0.1$ & $0.93 \pm 0.002$ & $9800 \pm 764$ & $12.0 \pm 0.03$ \\
\hline
\end{tabular}

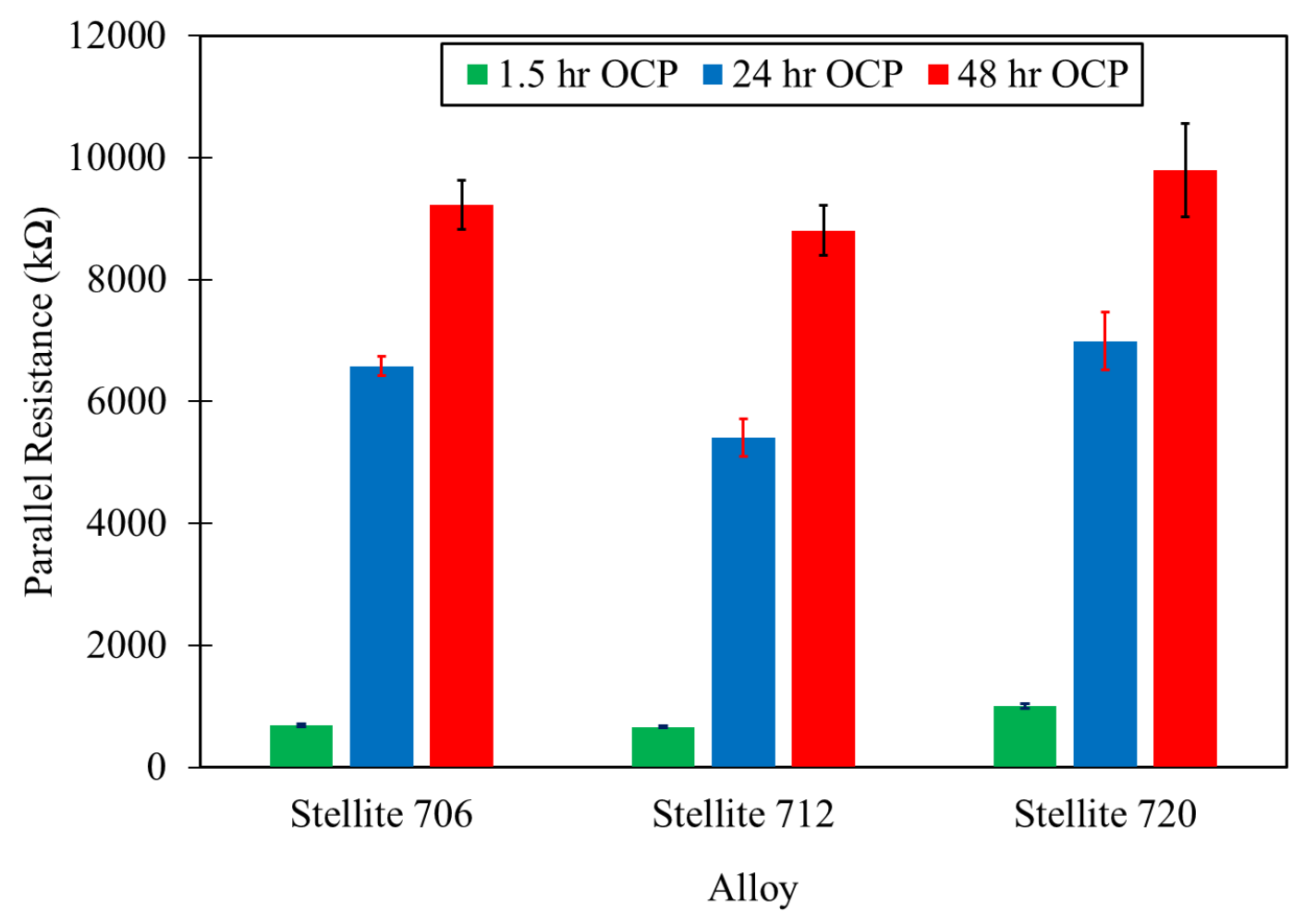

Figure 4-19: $R_{p}$ comparison for each OCP duration 


\subsubsection{Equivalent circuit}

Based on the results of the bode plot in Figure 4-7, there are three individual R-CPE parallel circuits, which describe the high, mid and low-frequency ranges. As described in Section 2.4.2, the measured capacitive response is not the same as an ideal capacitor due to the heterogeneity of the specimen surface, hence CPE is used to describe the capacitive behaviour. Figure 4-20 is the circuit representation of the system, which accurately works only up to $10^{4} \mathrm{~Hz}$ maximum.

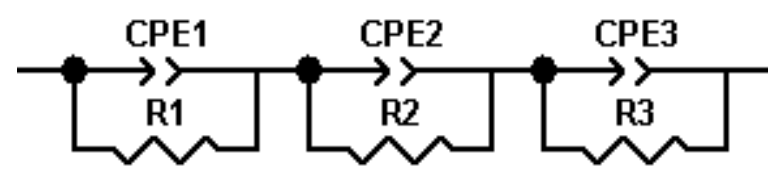

Figure 4-20: Initial equivalent circuit model for tested alloys

R-CPE1 circuit represents capacitance and resistance at high frequency from $10 \mathrm{kHz}$ and above, R-CPE2 circuit is for mid-frequency from $100-10 \mathrm{kHz}$ and R-CPE3 circuit is for low frequency below $100 \mathrm{~Hz}$. However, the CPE1 values have large errors associated with these circuits due to distortion and CPE2 cannot exist due to the mid-frequency being solution resistance. Thus, a final equivalent circuit model was proposed, as displayed in Figure 4-21 after considering all the corrections.

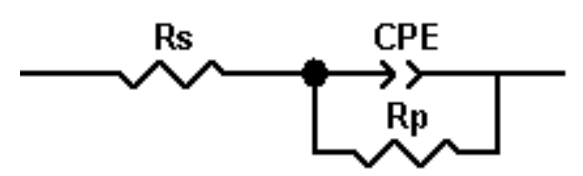

Figure 4-21: Final circuit model for tested alloys

This model is like a simple Randles circuit discussed in Section 2.4.2. It has only one R-CPE component, which describes the large semi-circles shown in Figure 4-22. If the semi-circles were to be drawn the entire way up to the end, they would look like as shown in Figure 2-17. 


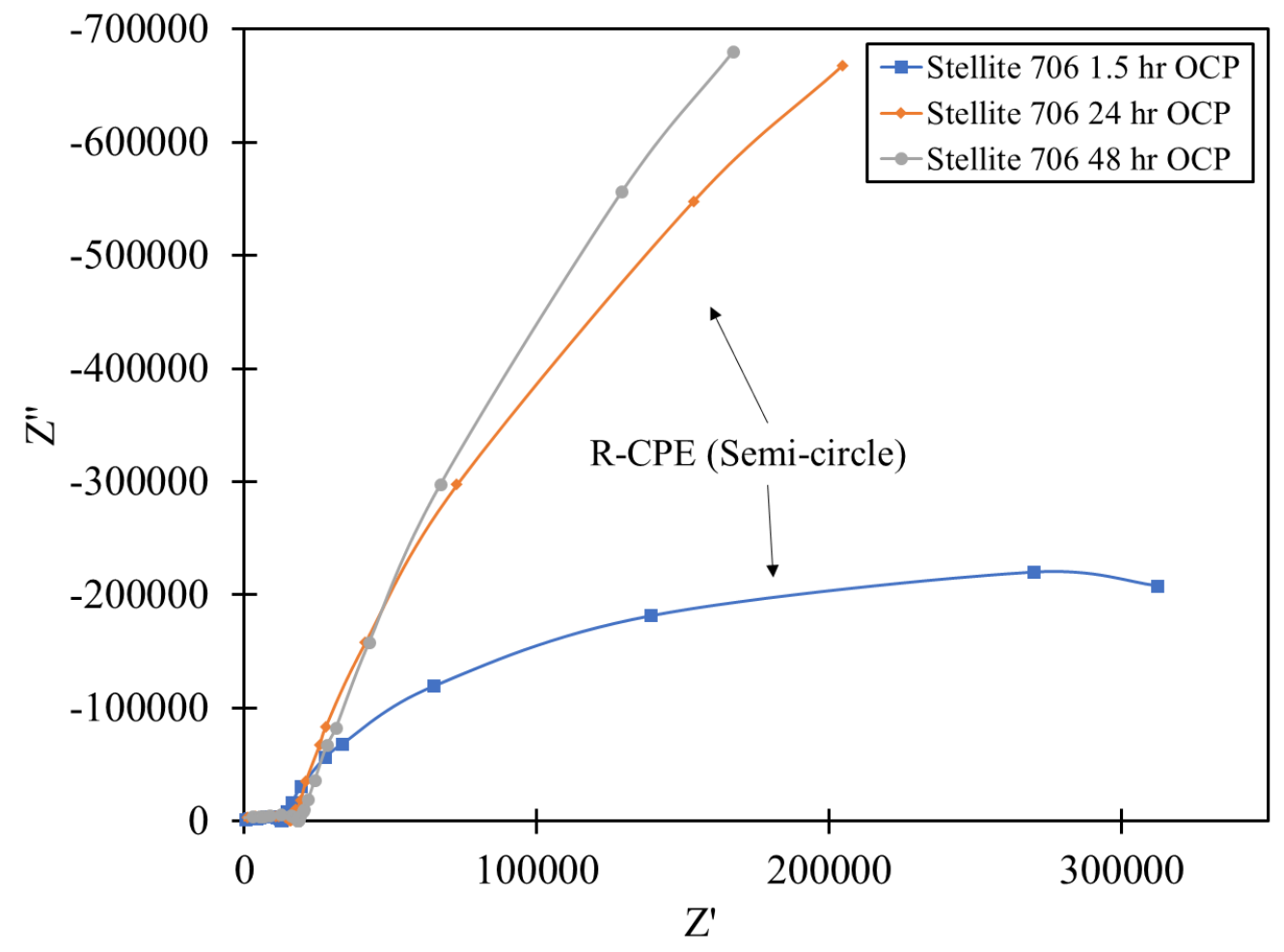

Figure 4-22: Nyquist plot of Stellite 706 for different OCP durations

\subsection{Polarization Results}

\subsubsection{Potentiodynamic polarization}

From what is observed quantitatively in EIS, the general corrosion behaviour should be analogous in cyclic polarization. The polarization forward curve of the three Stellite alloys has a similar trend from the start of the test to the peak potential value, as shown from Figure 4-23 to Figure 4-26. The test starts from $-0.5 \mathrm{~V}$ and after the turning point within the anodic region approximately between 0 to $0.3 \mathrm{~V}$, all alloys display a small spontaneous passive region. Beyond $0.3 \mathrm{~V}$ the curve crosses the $E_{p}$ where the curve transitioned to a transpassive mechanism, which indicates passive film breakdown and recovery simultaneously. The maximum current density reached is less than $10^{-4} \mathrm{~A}$. The forward scan stops after $1.2 \mathrm{~V}$ and the reverse scan starts, which is discussed in Section 4.3.2. 


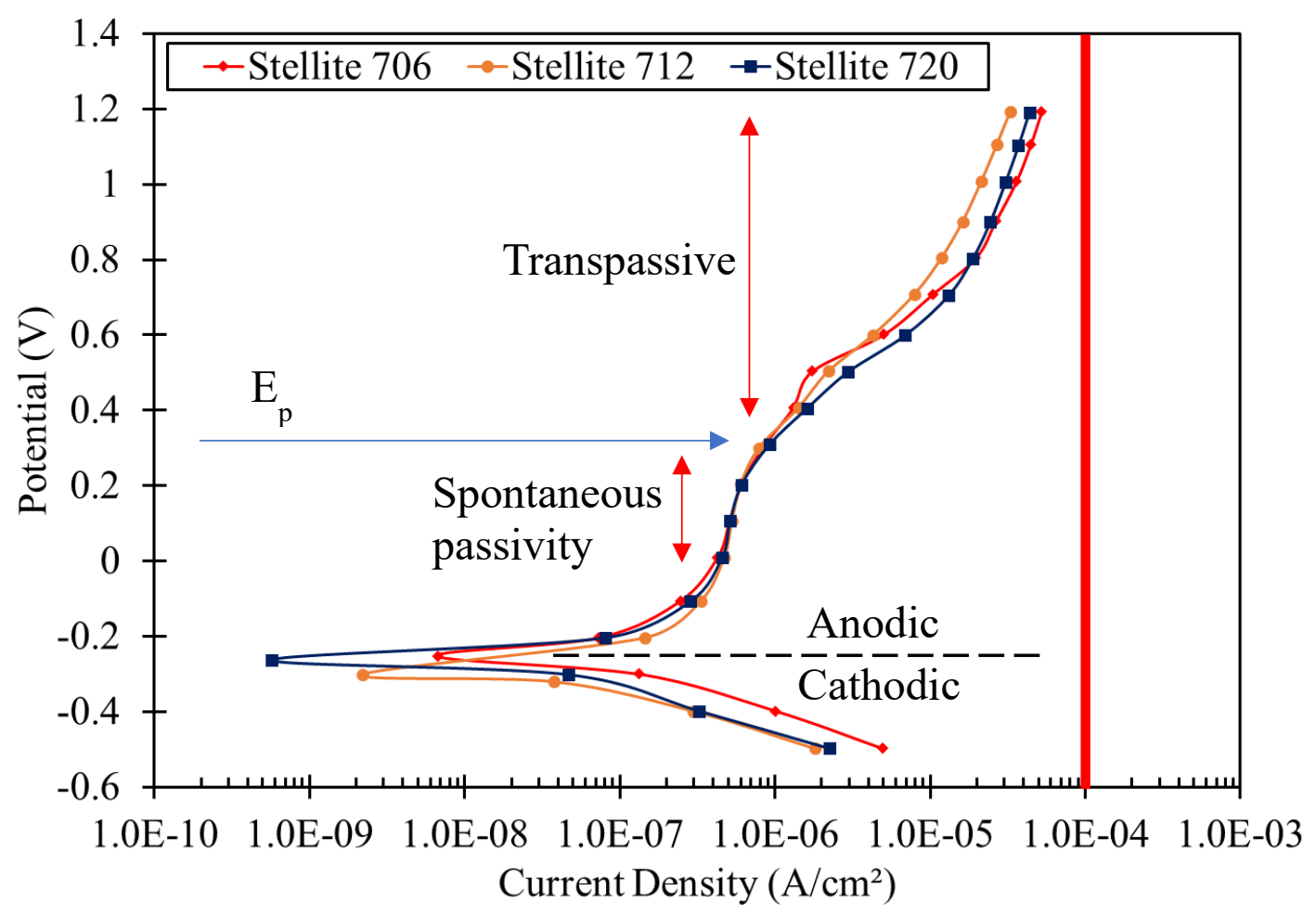

Figure 4-23: Polarization curves at $25^{\circ} \mathrm{C}$

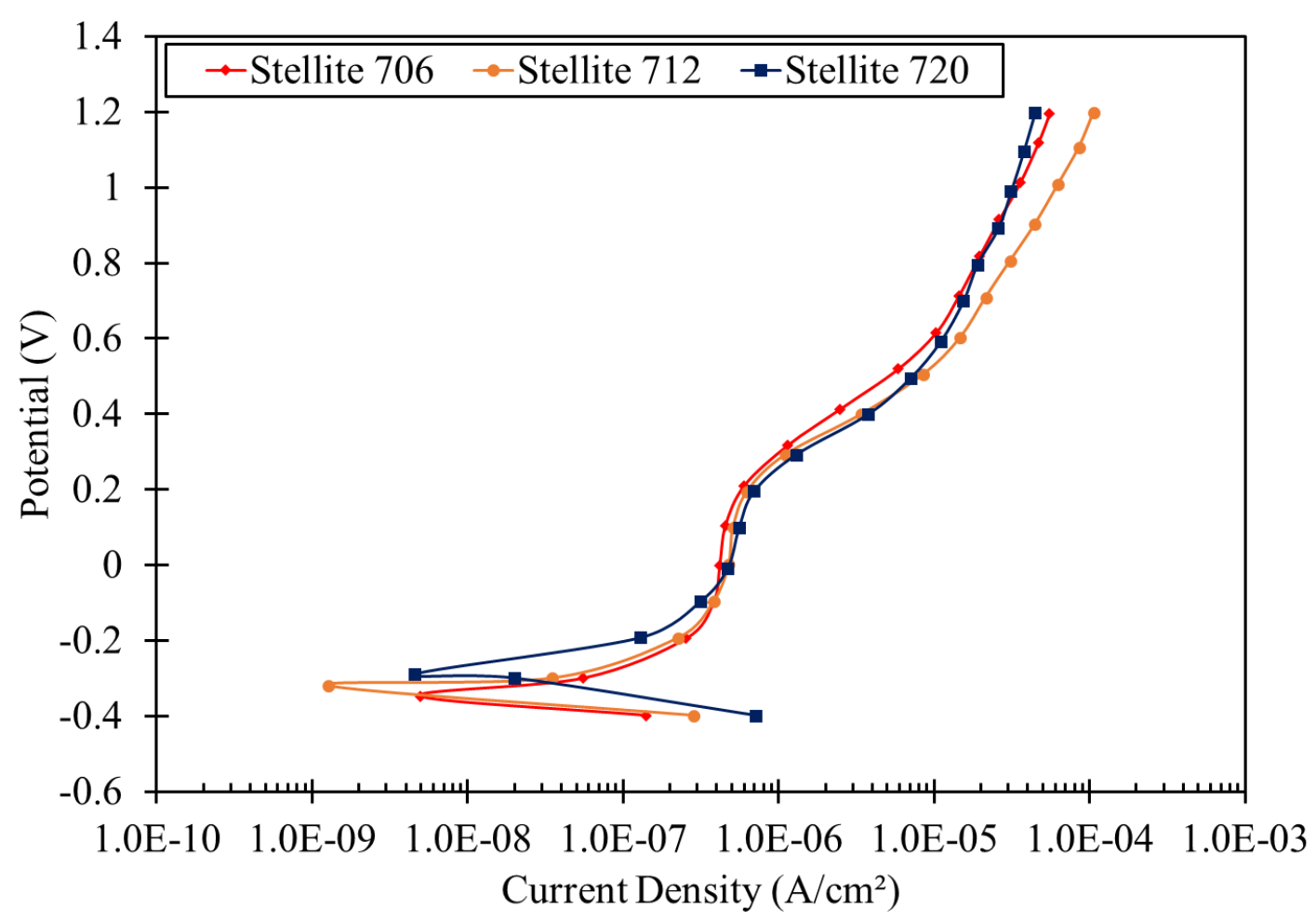

Figure 4-24: Polarization curves at $50^{\circ} \mathrm{C}$ 


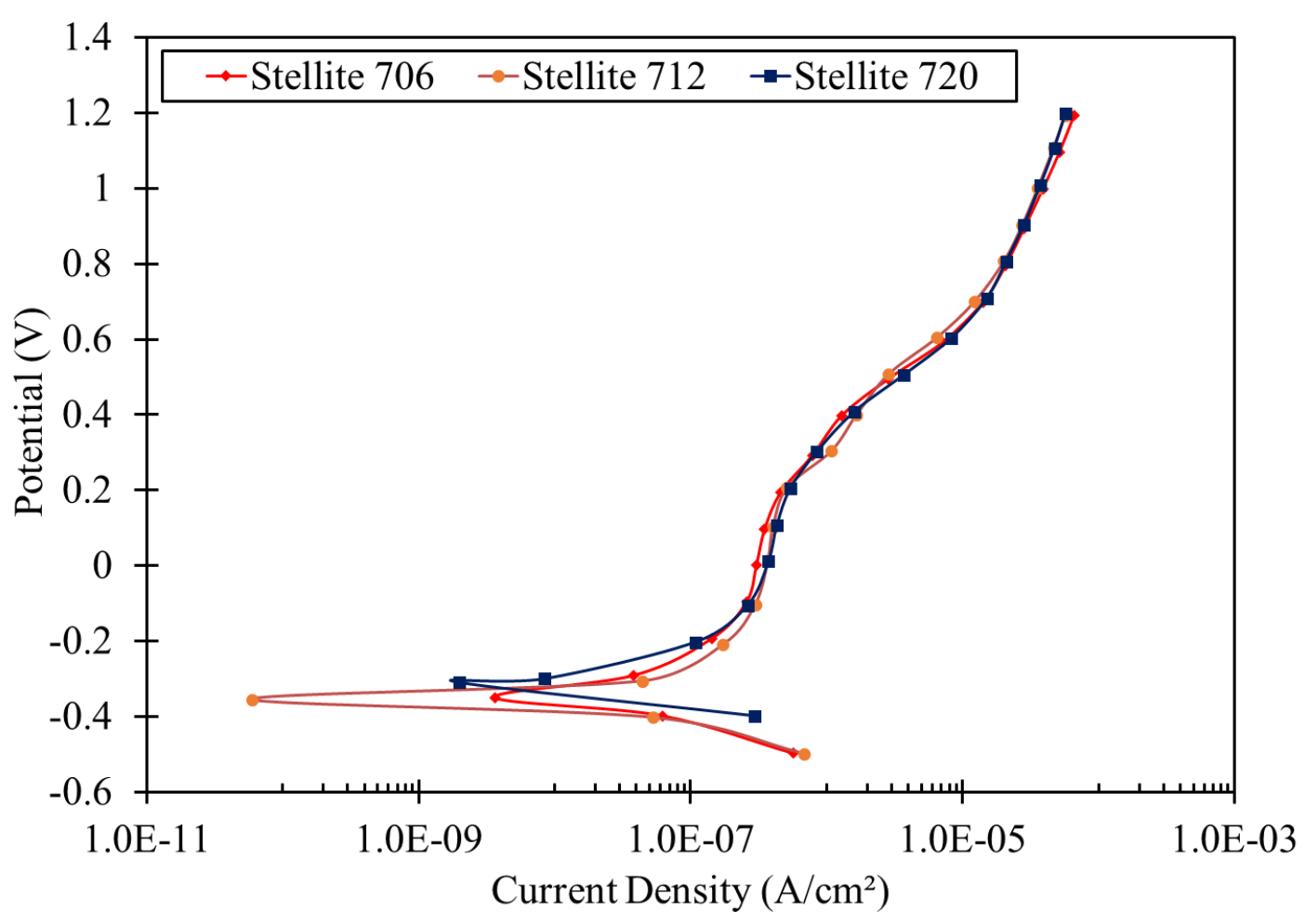

Figure 4-25: Polarization curves of worn alloys at $25^{\circ} \mathrm{C}$

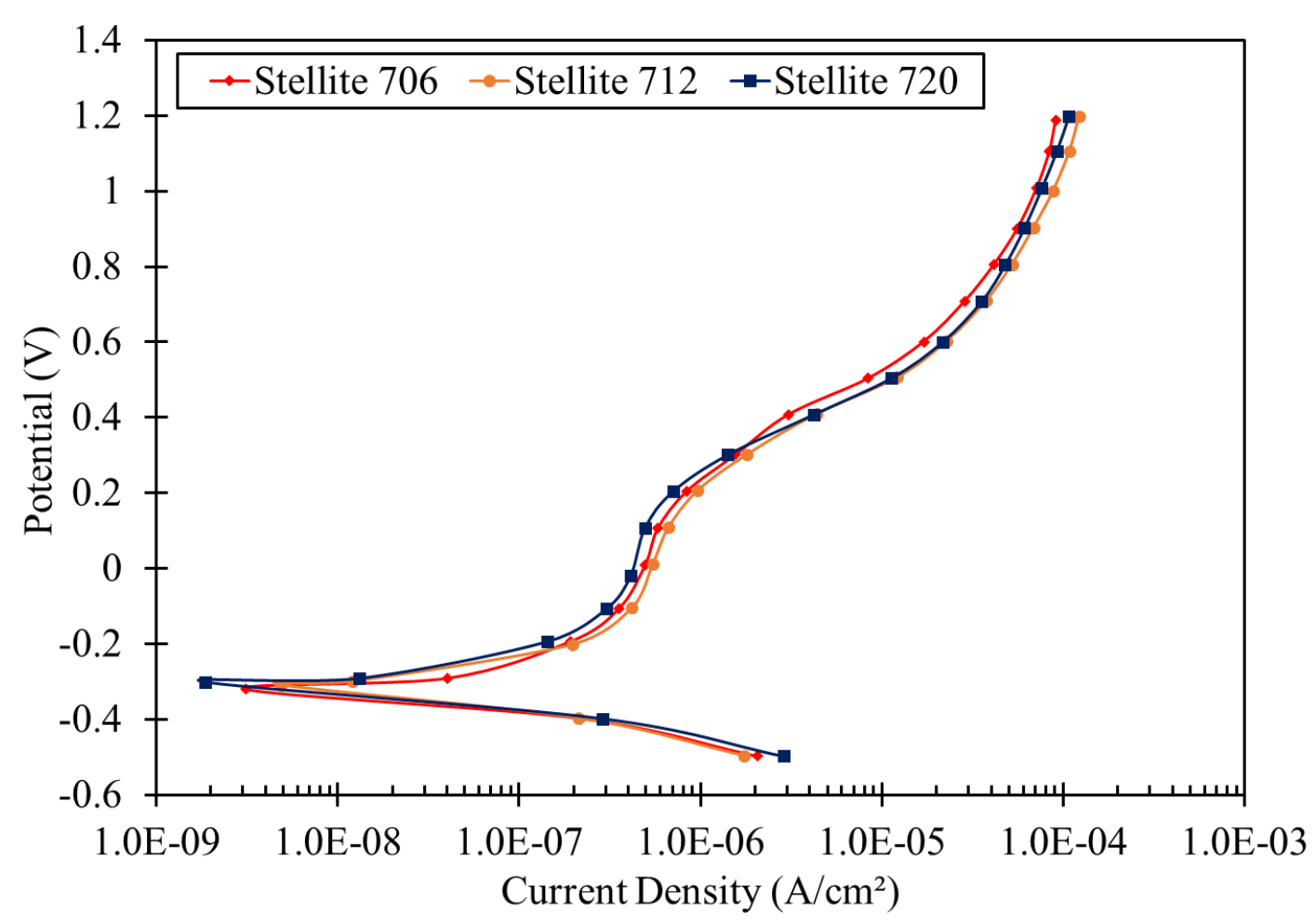

Figure 4-26: Polarization curves of worn alloys at $50^{\circ} \mathrm{C}$ 
Quantitatively the differences in general corrosion among the alloys are summarized in Table 4-3. If $E_{c o r r}$ is a more positive value with a small $I_{c o r r}$ and large $R_{p}$, then a material exhibits good corrosion resistance and vise-versa [11]. With a short OCP duration of 1.5 hours, the $E_{\text {corr }}$ values did not significantly change among the three Stellite alloys suggesting that within the 1.5 hours of immersion time they all had similar resulting stability. The $E_{\text {corr }}$ also closely resemble each alloy's respective stable OCP values. In general, Stellite 720 exhibited better general corrosion performance in morpholine at both temperature and surface conditions, apart from Stellite 706 having better performance at $50^{\circ} \mathrm{C}$ for both surface conditions.

The worn alloy surfaces experienced strain hardening of solid solution matrix and smashing of carbides, which may have altered the corrosion behaviour of the alloys. From the results obtained, the $E_{\text {corr }}$ values are slightly more negative compared to the intact specimens at $25^{\circ} \mathrm{C}$, which indicates that the damaged surfaces are slightly more prone to corrosion. The $I_{c o r r}$ and $R_{p}$ values in comparison to intact specimens at $25^{\circ} \mathrm{C}$ have better performance and a similar trend is seen at $50^{\circ} \mathrm{C}$. Looking at the bar graphs for $R_{p}$ in Figure 4-27 and Figure 4-28, Stellite 720 performed better at $25^{\circ} \mathrm{C}$ and Stellite 706 performed slightly better at $50^{\circ} \mathrm{C}$ for both surface conditions. The graphs are analogous to what is seen in EIS where the elevated temperature did influence resistance performance. From the data obtained, in $25^{\circ} \mathrm{C}$ Stellite 720 has better performance due to increased Mo content, however, this does not seem to be the case with $50^{\circ} \mathrm{C}$. Stellite 706 having the least number of interfaces due to low Mo and $\mathrm{C}$ content has a higher resistance at $50^{\circ} \mathrm{C}$. 
Table 4-3: Summary of polarization test results after $1.5 \mathrm{hr}$ OCP

\begin{tabular}{|c|c|c|c|c|c|}
\hline Temperature & Alloy & $\mathbf{O C P}(\mathbf{m V})$ & $\mathbf{E}_{\mathbf{c o r r}}(\mathbf{m V})$ & $\left.\mathbf{I}_{\mathbf{c o r r}} \mathbf{( n A} / \mathbf{c m}^{\mathbf{2}}\right)$ & $\mathbf{R}_{\mathbf{p}}\left(\mathbf{k} \mathbf{\Omega} \cdot \mathbf{c m}^{\mathbf{2}}\right)$ \\
\hline \multirow{3}{*}{$25^{\circ} \mathrm{C}$} & Stellite 706 & $-298 \pm 14$ & $-289 \pm 36$ & $47.1 \pm 2$ & $553 \pm 30$ \\
\cline { 2 - 6 } & Stellite 712 & $-300 \pm 27$ & $-308 \pm 14$ & $45.2 \pm 2$ & $577 \pm 28$ \\
\cline { 2 - 6 } & Stellite 720 & $-271 \pm 24$ & $-275 \pm 17$ & $27.8 \pm 4$ & $949 \pm 146$ \\
\hline \multirow{3}{*}{$50^{\circ} \mathrm{C}$} & Stellite 706 & $-349 \pm 17$ & $-338 \pm 33$ & $35.5 \pm 7$ & $771 \pm 95$ \\
\cline { 2 - 6 } & Stellite 712 & $-308 \pm 25$ & $-321 \pm 27$ & $54.8 \pm 6$ & $478 \pm 52$ \\
\cline { 2 - 6 } & Stellite 720 & $-270 \pm 25$ & $-298 \pm 17$ & $44.1 \pm 5$ & $595 \pm 77$ \\
\hline \multirow{3}{*}{$25^{\circ} \mathrm{C}$} & Worn Stellite 706 & $-312 \pm 13$ & $-334 \pm 16$ & $28.1 \pm 2$ & $956 \pm 49$ \\
\cline { 2 - 6 } & Worn Stellite 712 & $-317 \pm 15$ & $-336 \pm 15$ & $27.0 \pm 1$ & $995 \pm 53$ \\
\cline { 2 - 6 } & Worn Stellite 720 & $-301 \pm 30$ & $-319 \pm 31$ & $23.3 \pm 0.5$ & $1138 \pm 21$ \\
\hline \multirow{3}{*}{$50^{\circ} \mathrm{C}$} & Worn Stellite 706 & $-296 \pm 25$ & $-319 \pm 15$ & $36.0 \pm 1$ & $723 \pm 20$ \\
\cline { 2 - 6 } & Worn Stellite 712 & $-293 \pm 3$ & $-309 \pm 3$ & $41.7 \pm 1$ & $625 \pm 16$ \\
\cline { 2 - 6 } & Worn Stellite 720 & $-312 \pm 33$ & $-325 \pm 30$ & $38.3 \pm 3$ & $680 \pm 46$ \\
\hline
\end{tabular}

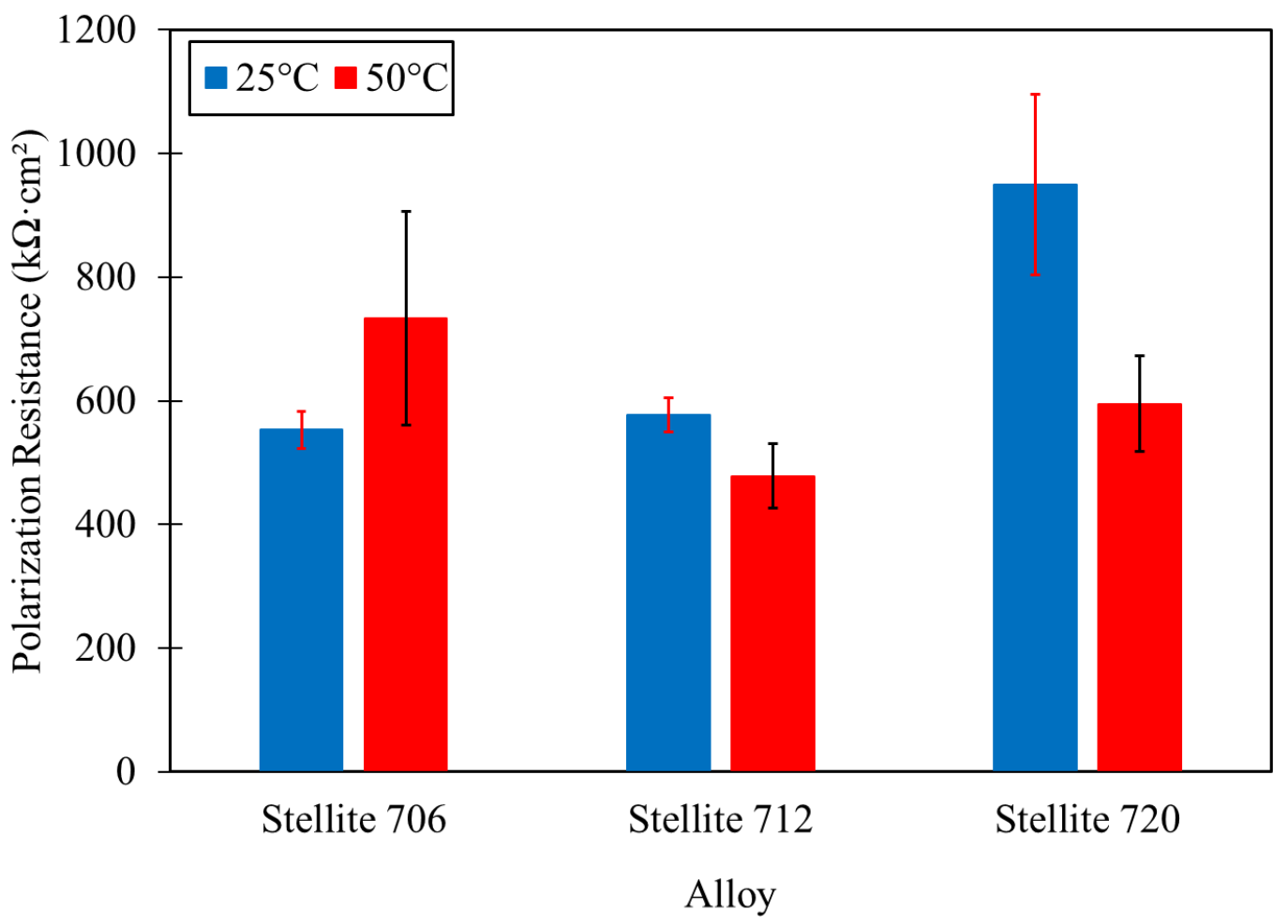

Figure 4-27: Comparison of $\boldsymbol{R}_{p}$ value for intact specimens 


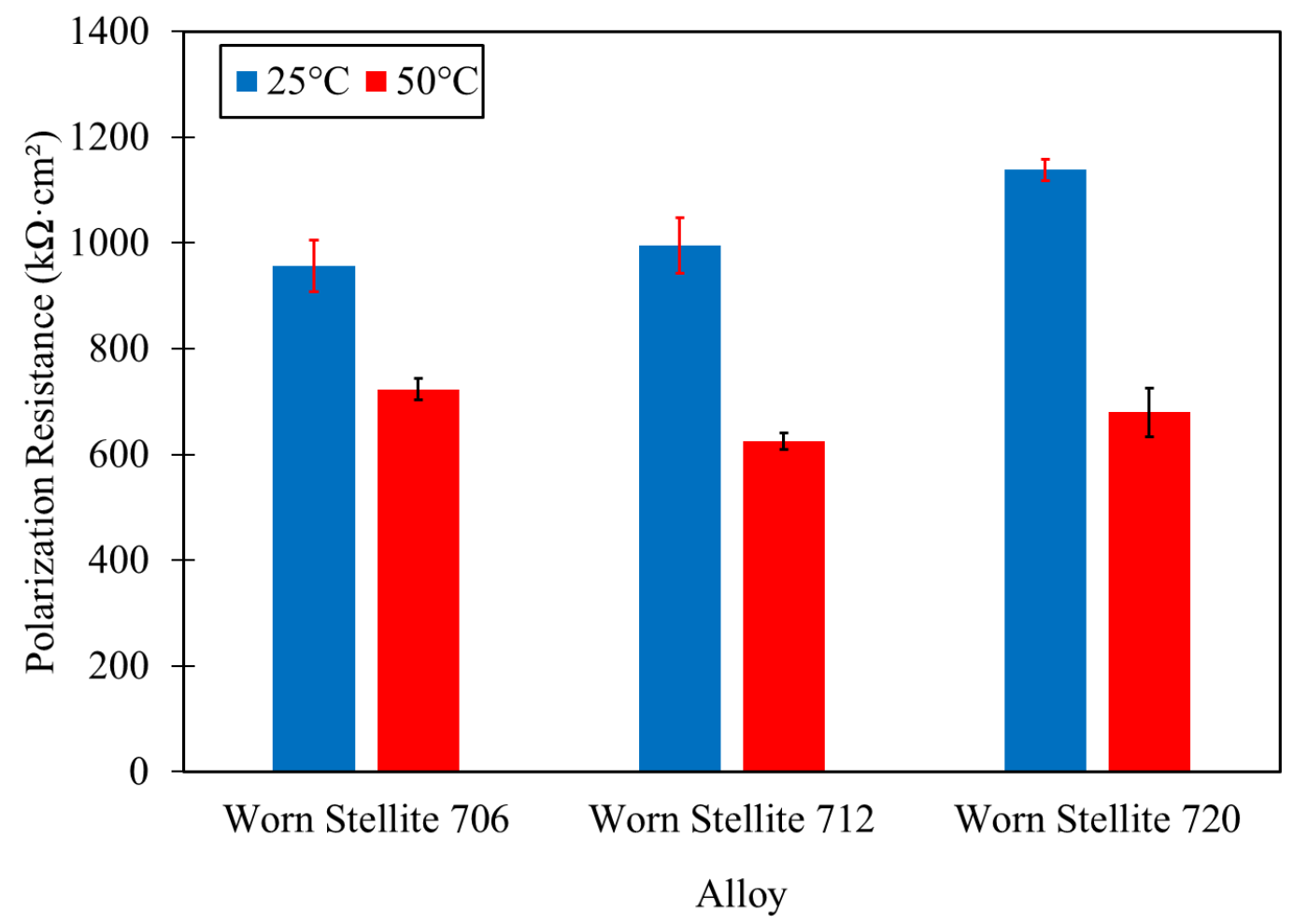

Figure 4-28: Comparison of $R_{p}$ value for worn specimens

For higher OCP durations, qualitatively the polarization curves appear to be similar compared to 1.5 hours OCP as shown in Figure 4-29 and Figure 4-30. However, quantitatively noticeable differences are found, as summarized in Table 4-4 where the $R_{p}$ values in comparison are much larger. The differences among the different OCP durations are once again analogous to what is observed for $R_{p}$ in the EIS results. A bar graph is shown in Figure 4-31 effectively comparing the $R_{p}$ values and clearly showing that Stellite 720 in each OCP duration has a higher resistance. The $E_{\text {corr }}$ values can now be effectively compared where as expected it becomes more positive with higher OCP duration signifying that the resistive properties of the alloys improved. 


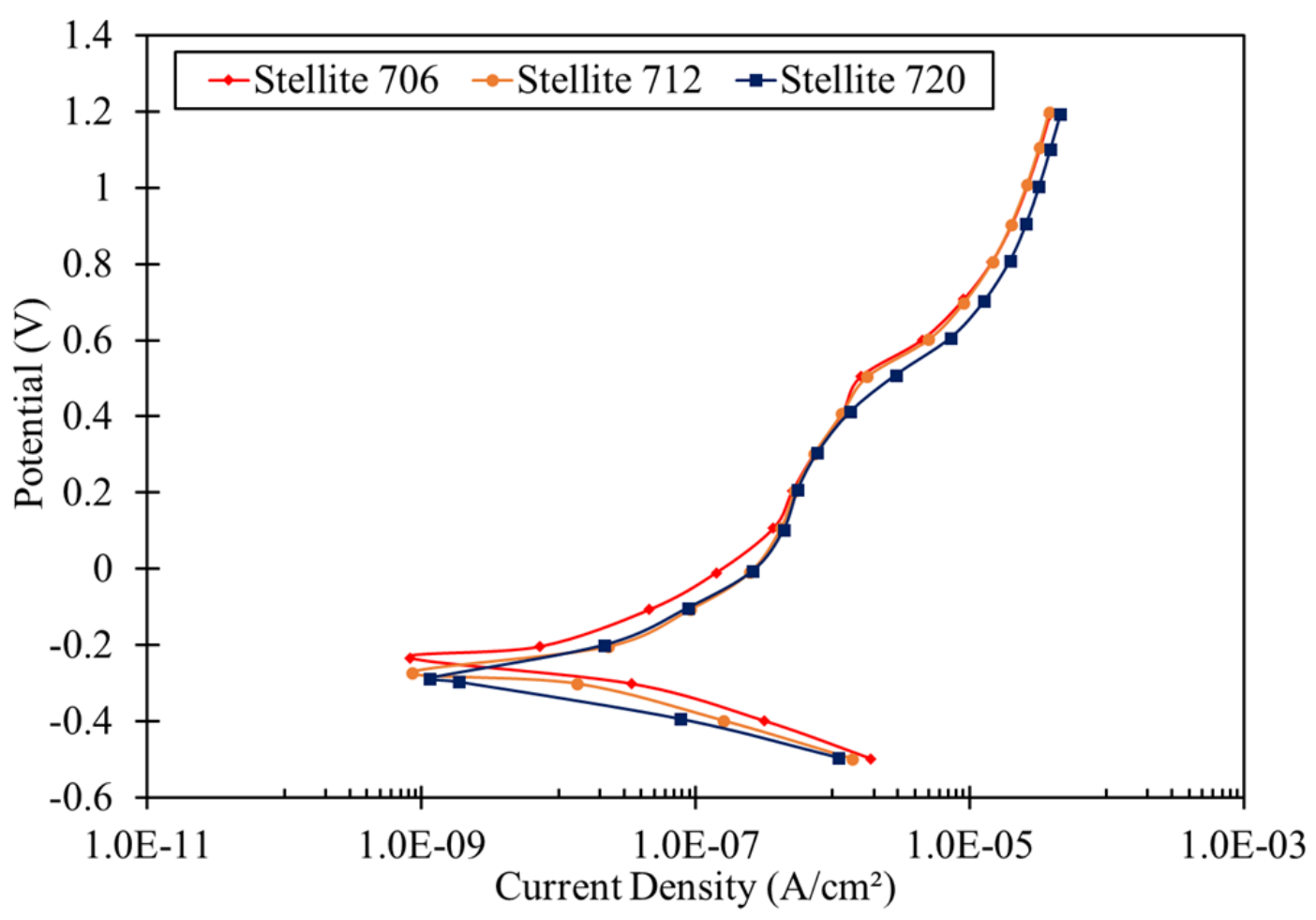

Figure 4-29: $24 \mathrm{hr}$ OCP polarization plot for Stellite alloys at $25^{\circ} \mathrm{C}$

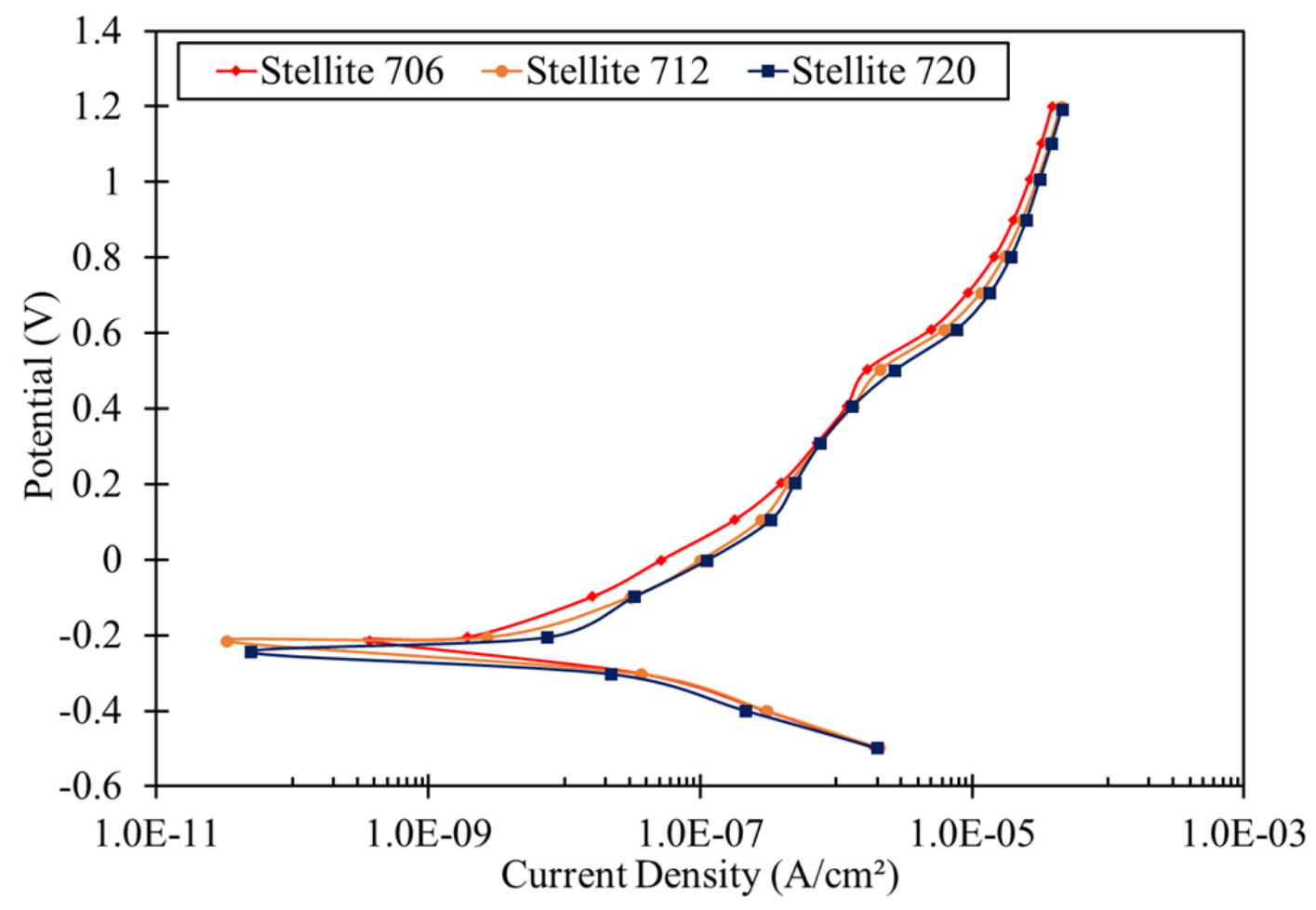

Figure 4-30: $48 \mathrm{hr}$ OCP polarization plot for Stellite alloys at $25^{\circ} \mathrm{C}$ 
Table 4-4: Summary of polarization parameters for all OCP durations

\begin{tabular}{|c|c|c|c|c|c|}
\hline Alloy & Time (hr) & OCP $(\mathbf{m V})$ & $\mathbf{E}_{\text {corr }} \mathbf{( m V )}$ & $\left.\mathbf{I}_{\mathbf{c o r r}} \mathbf{( n A / \mathbf { c m } ^ { 2 }}\right)$ & $\mathbf{R}_{\mathbf{p}}\left(\mathbf{k} \mathbf{\mathbf { c }} \mathbf{c m}^{\mathbf{2}}\right)$ \\
\hline \multirow{3}{*}{ Stellite 706 } & 1.5 & $-298 \pm 14$ & $-289 \pm 36$ & $47.1 \pm 2$ & 553 \\
\cline { 2 - 6 } & 24 & $-223 \pm 22$ & $-252 \pm 27$ & $9.3 \pm 0.1$ & 2807 \\
\cline { 2 - 6 } & 48 & $-179 \pm 28$ & $-224 \pm 14$ & $6.1 \pm 0.2$ & 4245 \\
\hline \multirow{3}{*}{ Stellite 712 } & 1.5 & $-300 \pm 27$ & $-308 \pm 14$ & $45.2 \pm 2$ & 577 \\
\cline { 2 - 6 } & 24 & $-259 \pm 13$ & $-272 \pm 1$ & $8.7 \pm 0.1$ & 2967 \\
\cline { 2 - 6 } & 48 & $-210 \pm 20$ & $-246 \pm 39$ & $5.7 \pm 0.04$ & 4607 \\
\hline \multirow{3}{*}{ Stellite 720 } & 1.5 & $-271 \pm 24$ & $-275 \pm 17$ & $27.4 \pm 5$ & 949 \\
\cline { 2 - 6 } & 24 & $-250 \pm 15$ & $-297 \pm 1$ & $7.0 \pm 0.1$ & 3713 \\
\cline { 2 - 6 } & 48 & $-211 \pm 24$ & $-247 \pm 20$ & $5.0 \pm 0.05$ & 5229 \\
\hline
\end{tabular}

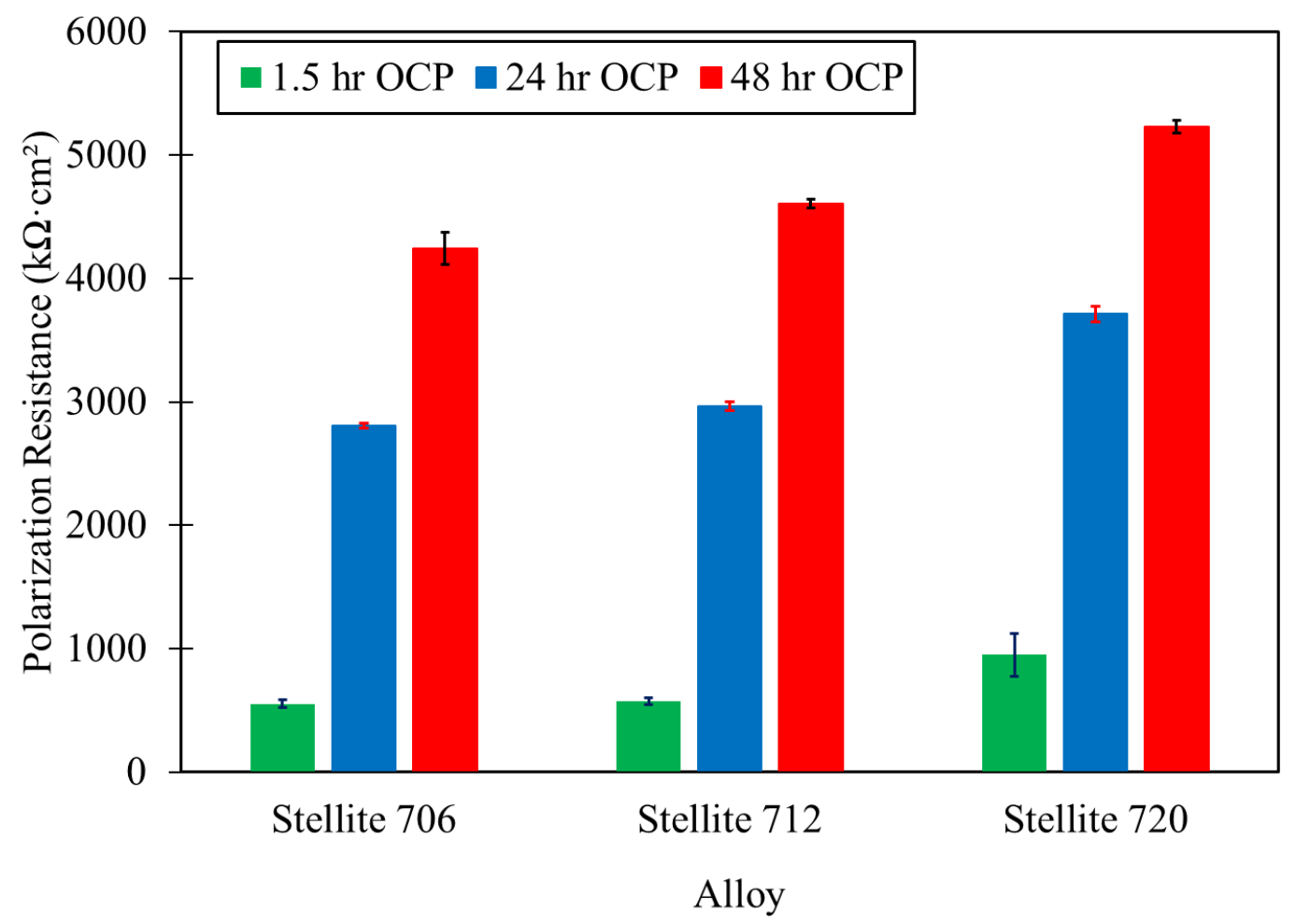

Figure 4-31: Comparison of $\boldsymbol{R}_{p}$ value for each OCP duration

\subsubsection{Hysteresis loop}

Localized corrosion resistance is qualitatively observed by looking at the reverse scan in cyclic polarization from the onset of the peak potential value. The peak potential value is $1.2 \mathrm{~V}$ and ends 
at $-0.3 \mathrm{~V}$. The main qualitative observation is within the entire transpassive region starting from the peak potential value down to near the $E_{p}$ starting point. The reverse scan is denoted as dotted lines as seen from Figure 4-32 to Figure 4-35. From the reverse scan curves of these alloys, it was observed to be a stable hysteresis behaviour as the reverse scan is either close or overlaps the forward scan. This is attributed to the good stability mechanism of the oxide layer on the specimen surface as the potential became more positive and swept backward. With a stable hysteresis behaviour, the three Stellite alloys have extremely small susceptibility to pitting in morpholine given the conditions in which they were tested in.

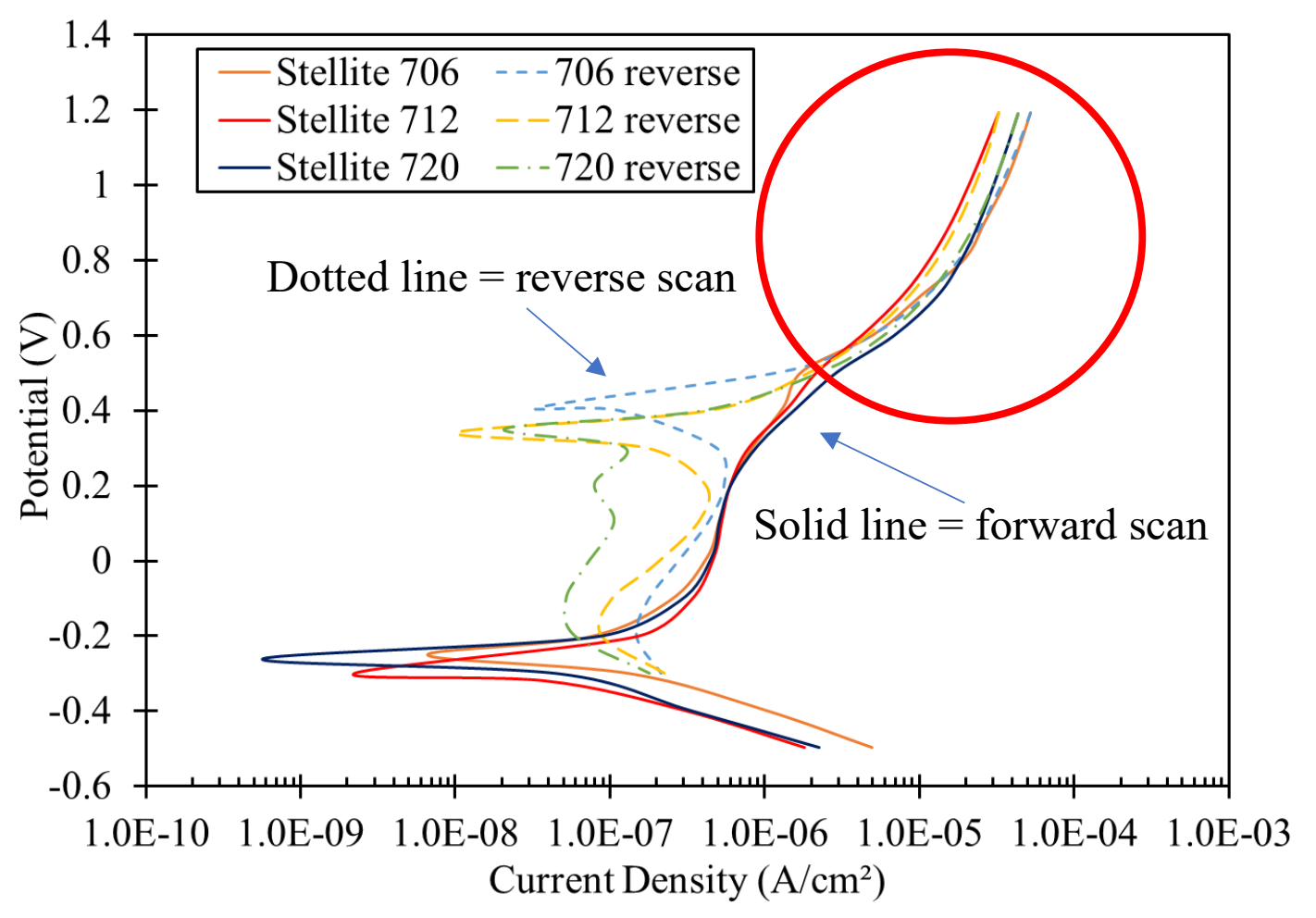

Figure 4-32: Hysteresis loop for intact Stellite alloys at $25^{\circ} \mathrm{C}$ 


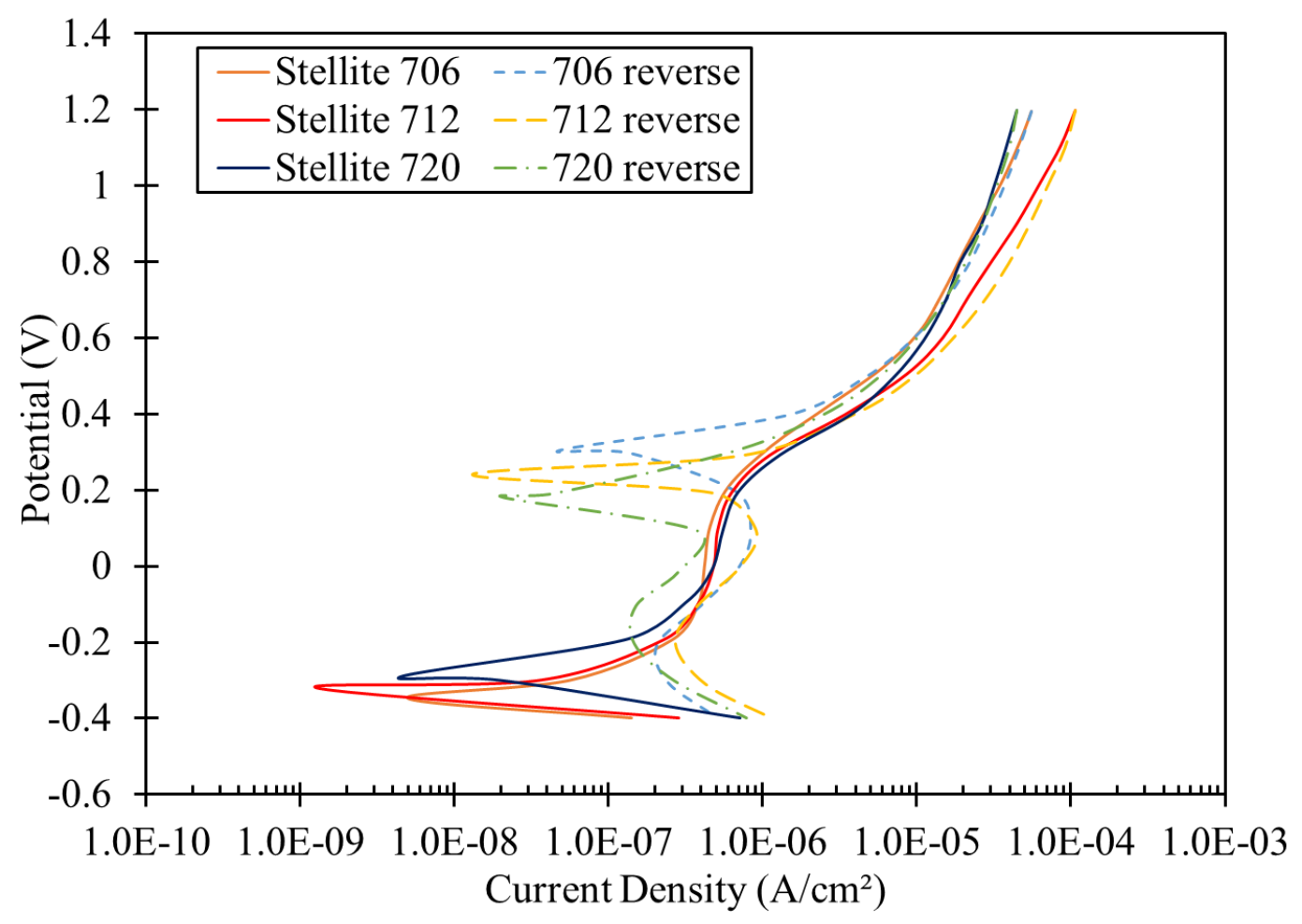

Figure 4-33: Hysteresis loop for intact Stellite alloys at $50^{\circ} \mathrm{C}$

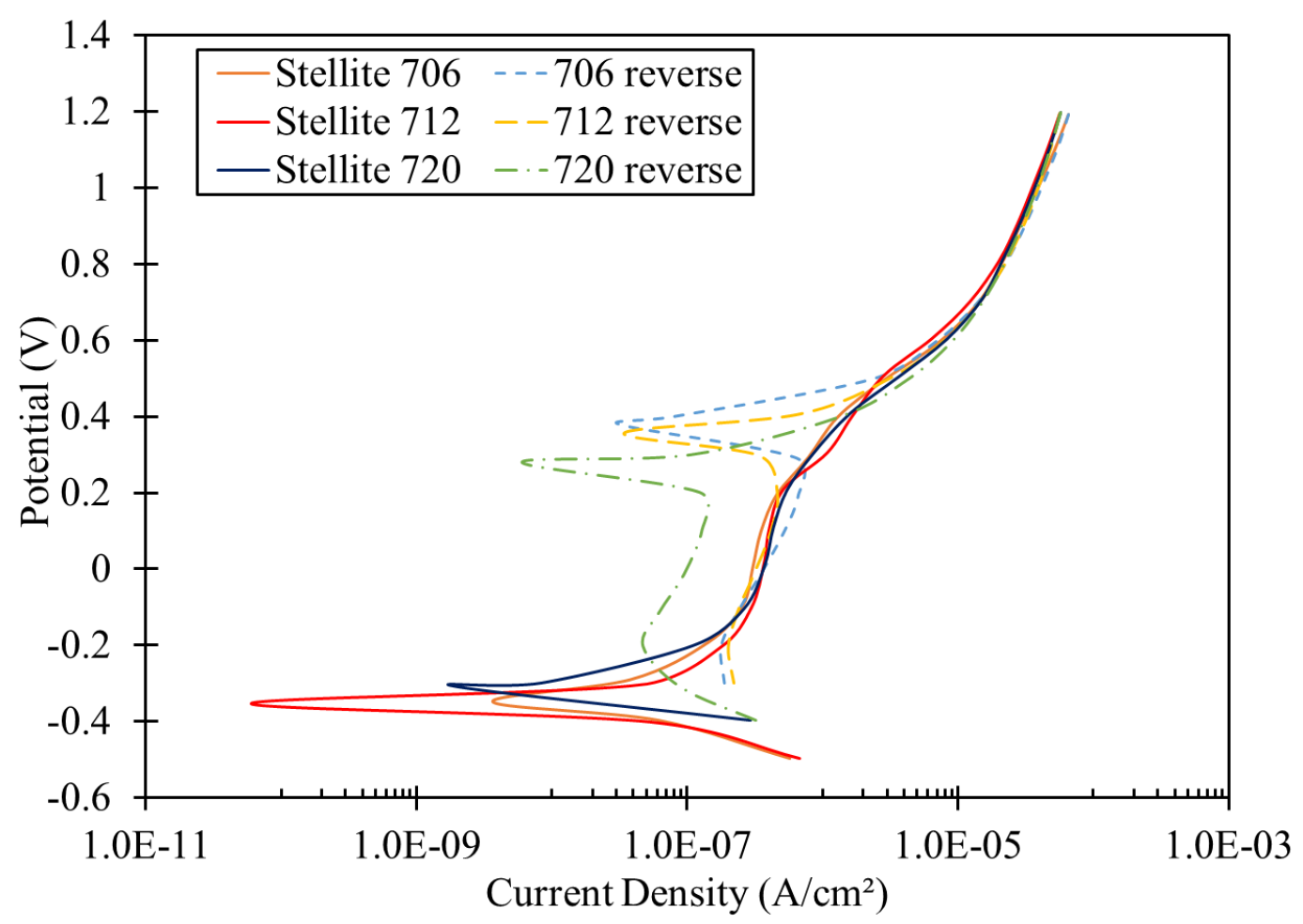

Figure 4-34: Hysteresis loop for worn Stellite alloys at $25^{\circ} \mathrm{C}$ 


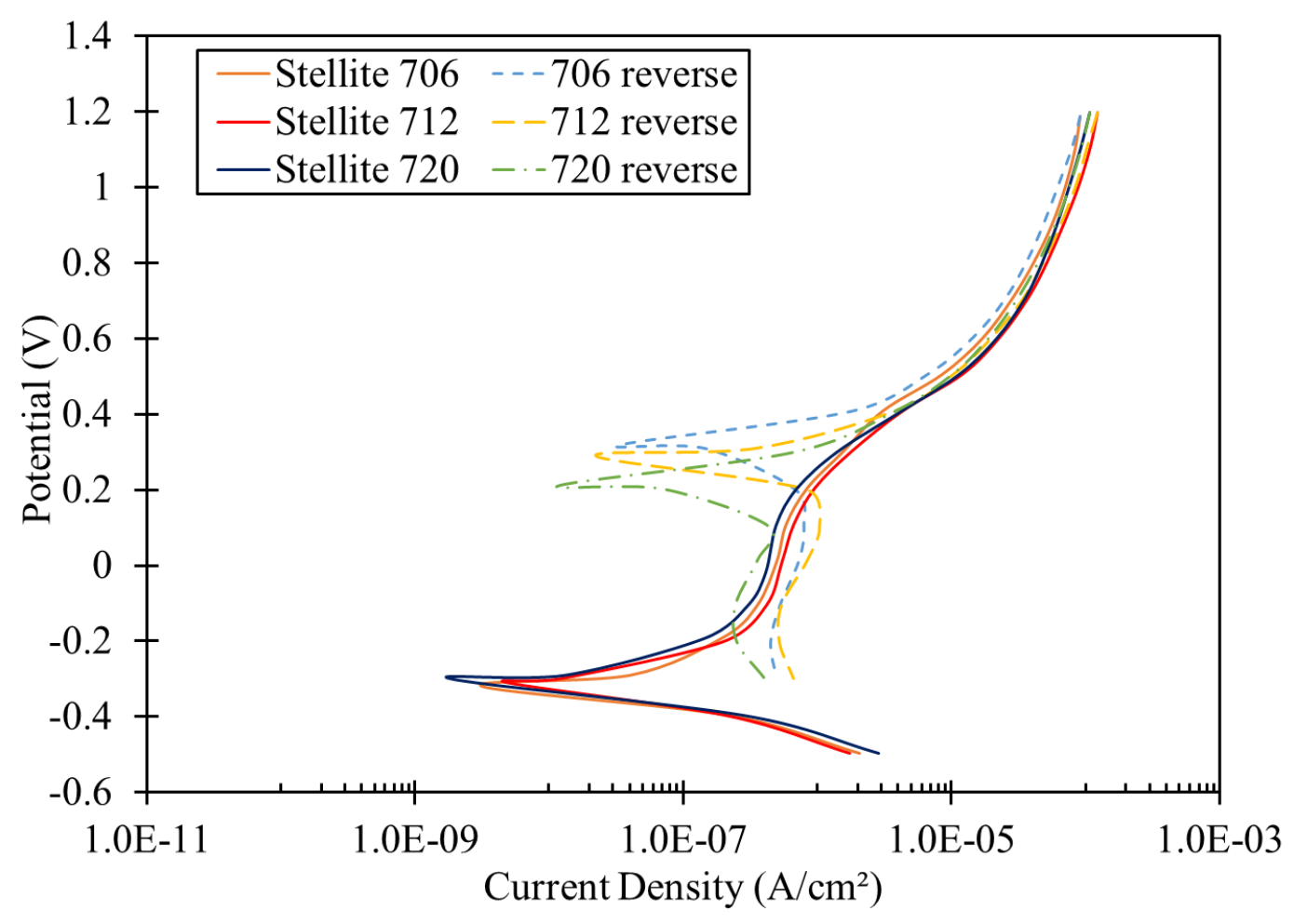

Figure 4-35: Hysteresis loop for worn Stellite alloys at $50^{\circ} \mathrm{C}$

The reverse scan results for 24 and 48 hours OCP is given in Figure 4-36 and Figure 4-37. The hysteresis behaviour was stable, and no changes were observed. Therefore, regardless of the OCP duration, the localized corrosion resistance behaviour for all three Stellite 700 series alloys display the same behaviour in morpholine at $\mathrm{pH} 9.5$. 


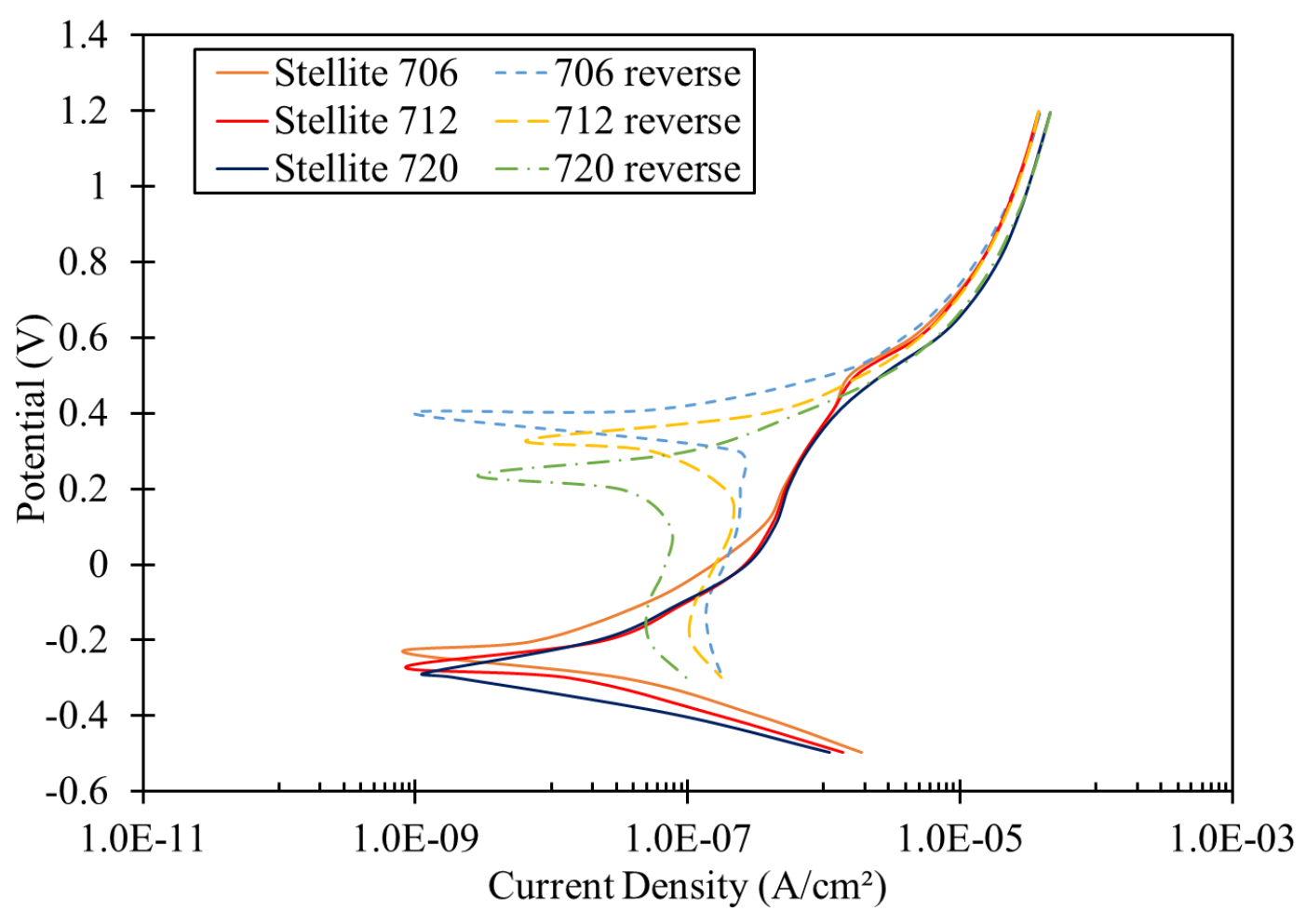

Figure 4-36: $24 \mathrm{hr}$ OCP hysteresis loop for intact Stellite alloys at $25^{\circ} \mathrm{C}$

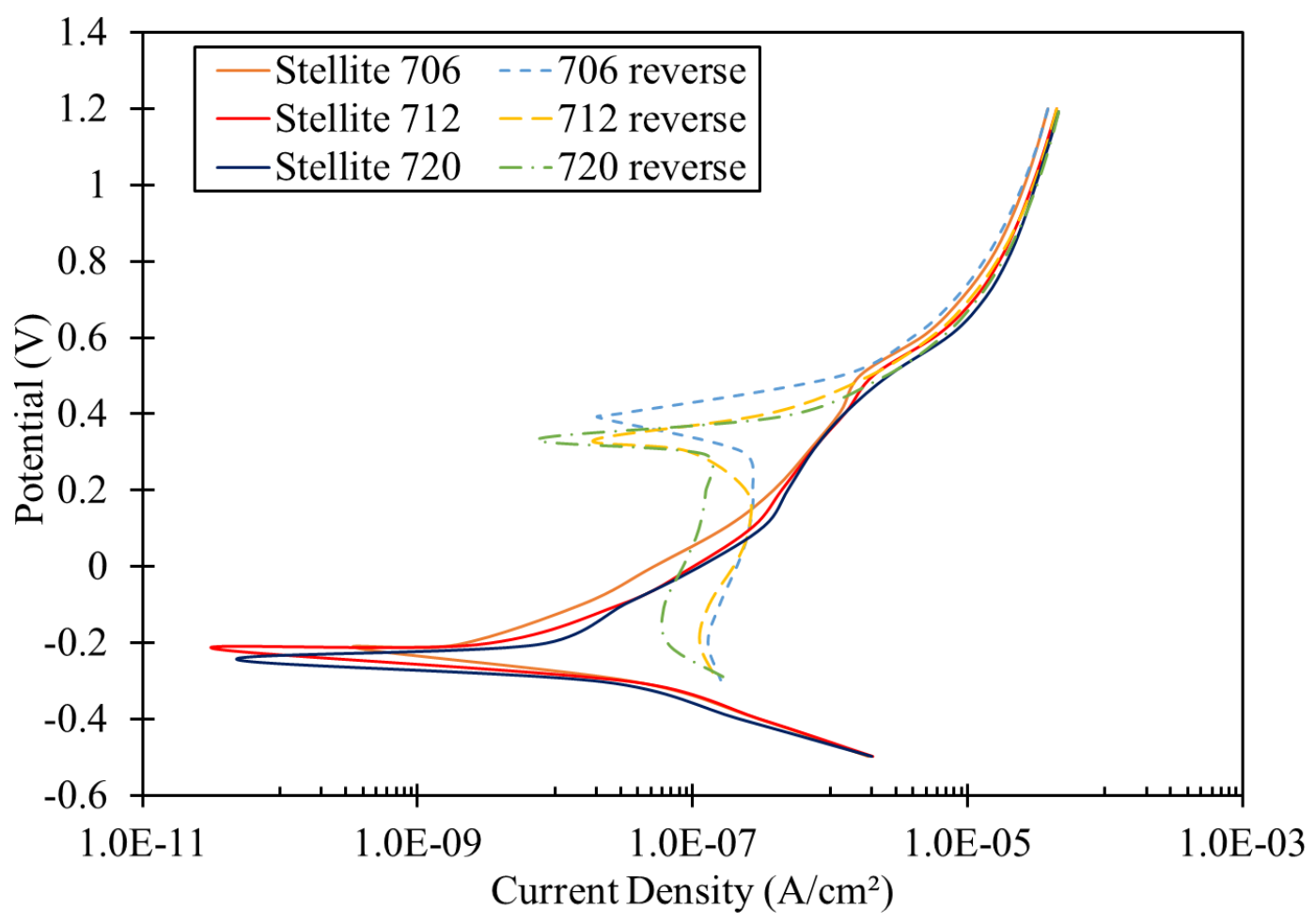

Figure 4-37: $48 \mathrm{hr}$ OCP hysteresis loop for intact Stellite alloys at $25^{\circ} \mathrm{C}$ 


\subsection{Corroded Surface}

The alloy specimens after the corrosion tests were examined using SEM and analyzed with EDS. The SEM images of the corroded surface at $25^{\circ} \mathrm{C}$ along with the intact surface of each alloy are given in Figure 4-38 to Figure 4-40. The surface morphology after corrosion is clearly seen, where the oxide film is noticeably fractured across the matrix. Closer magnification images are provided from Figure 4-41 to Figure 4-43, showing the fractured oxide layers on the surface. Initially, the assumption was that the fractured regions are grain boundaries, however, when a surface is corroded grain boundaries are not usually seen. This was confirmed in Figure 4-43, where the same fractured region is observed on the surface of the oxidized Cr carbide phase. In Stellite 720, the matrix is predominantly a mixture of $\mathrm{Co}, \mathrm{Cr}$ and Mo where the oxide fractured regions can be seen, however, they are extremely small.

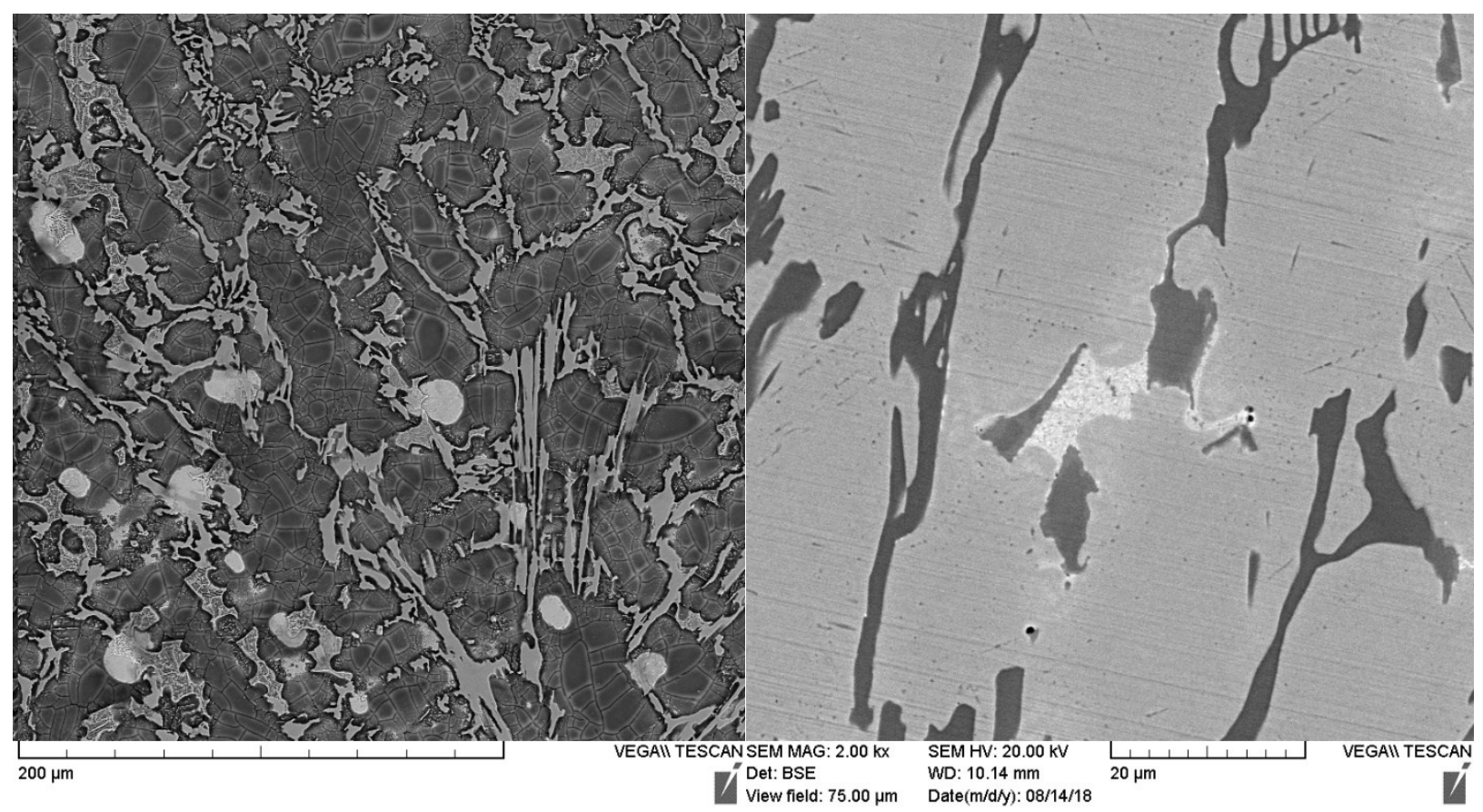

(a)

(b)

Figure 4-38: SEM images of Stellite 706: (a) corroded surface in morpholine solution with $\mathrm{pH} 9.5$ at $25^{\circ} \mathrm{C}$ and (b) intact surface 


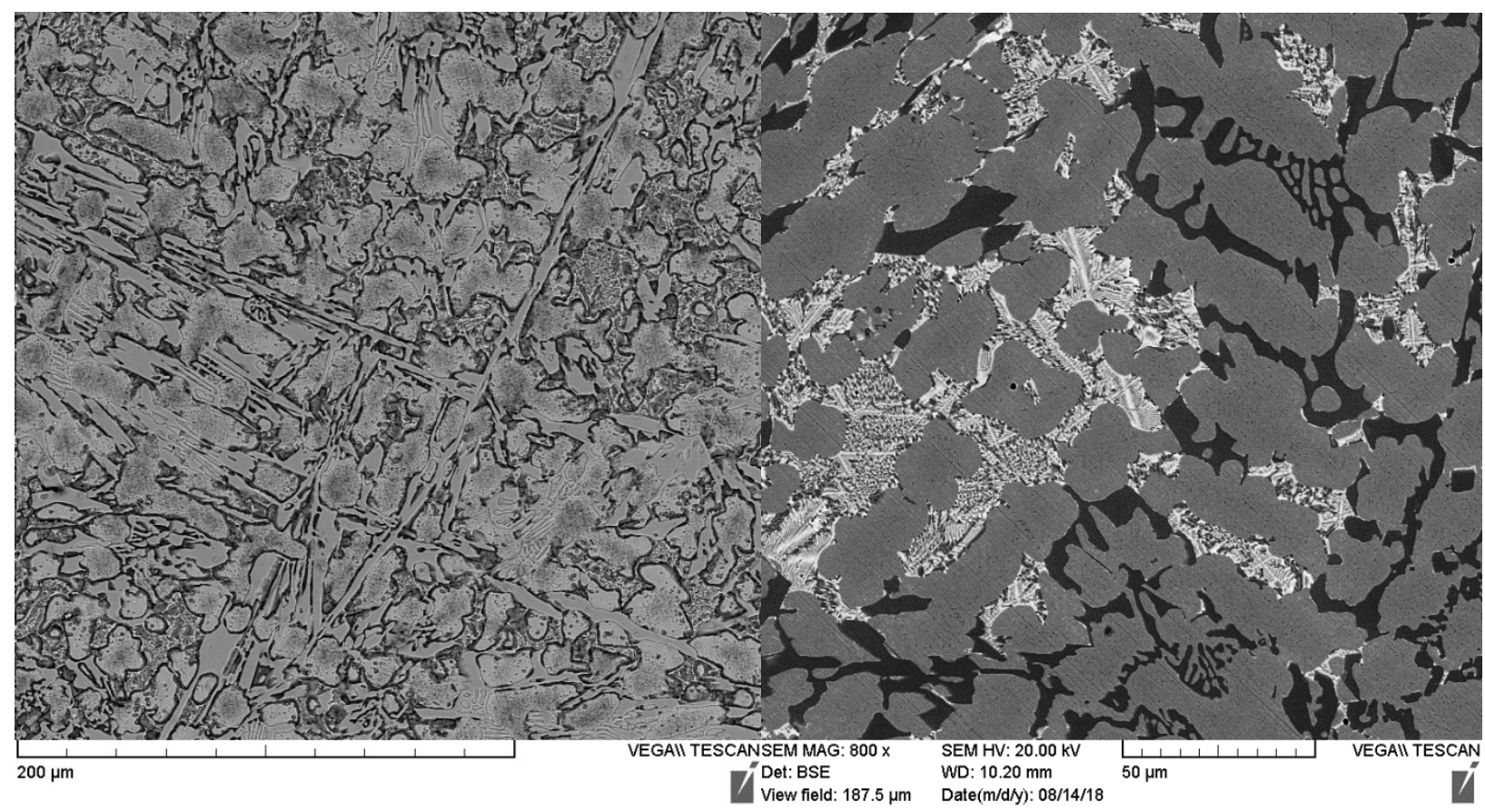

(a)

(b)

Figure 4-39: SEM images of Stellite 712: (a) corroded surface in morpholine solution with $\mathrm{pH} 9.5$ at $25^{\circ} \mathrm{C}$ and (b) intact surface

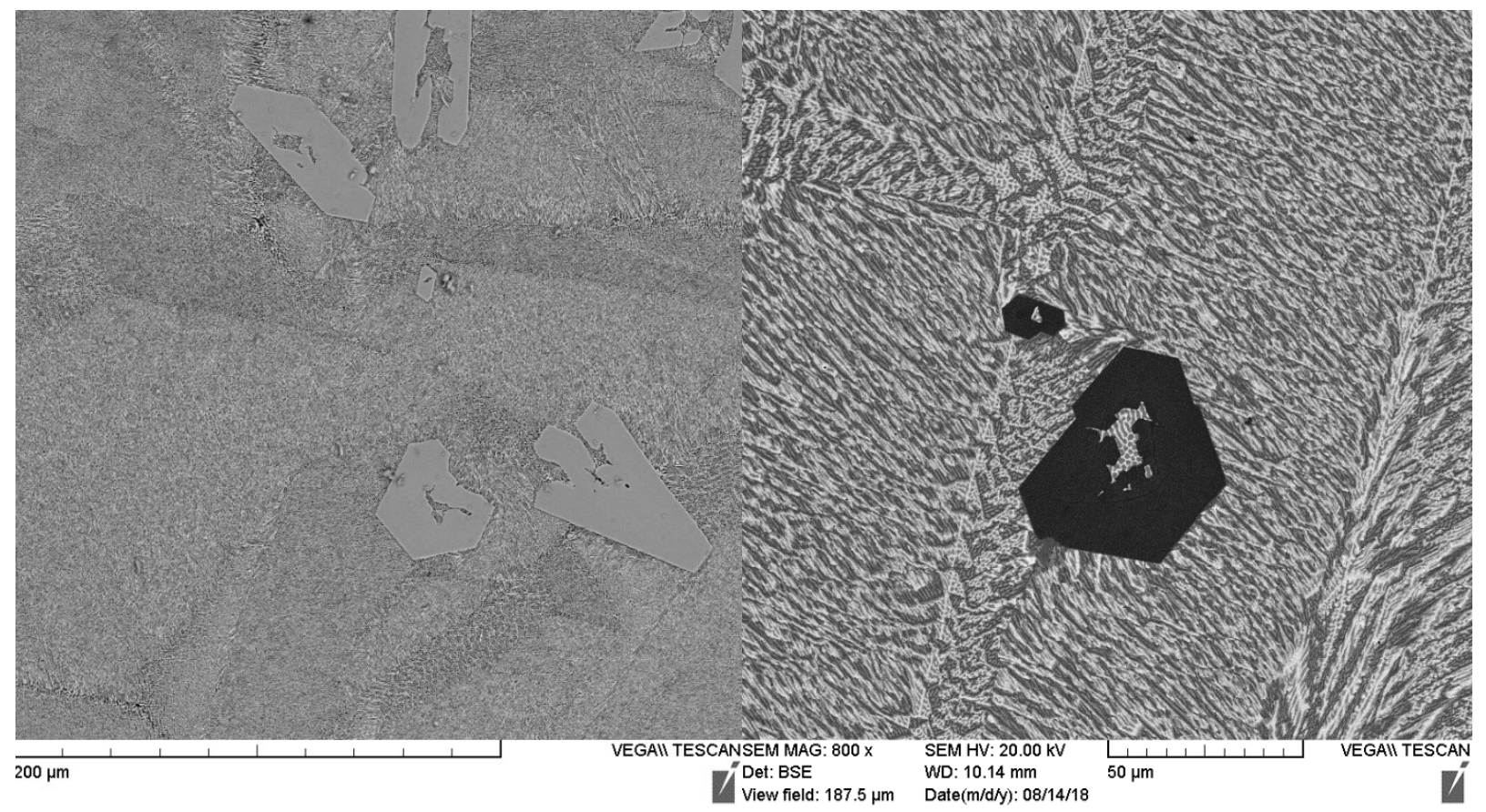

(a)

(b)

Figure 4-40: SEM images of Stellite 720: (a) corroded surface in morpholine solution with pH 9.5 at $25^{\circ} \mathrm{C}$ and (b) intact surface 


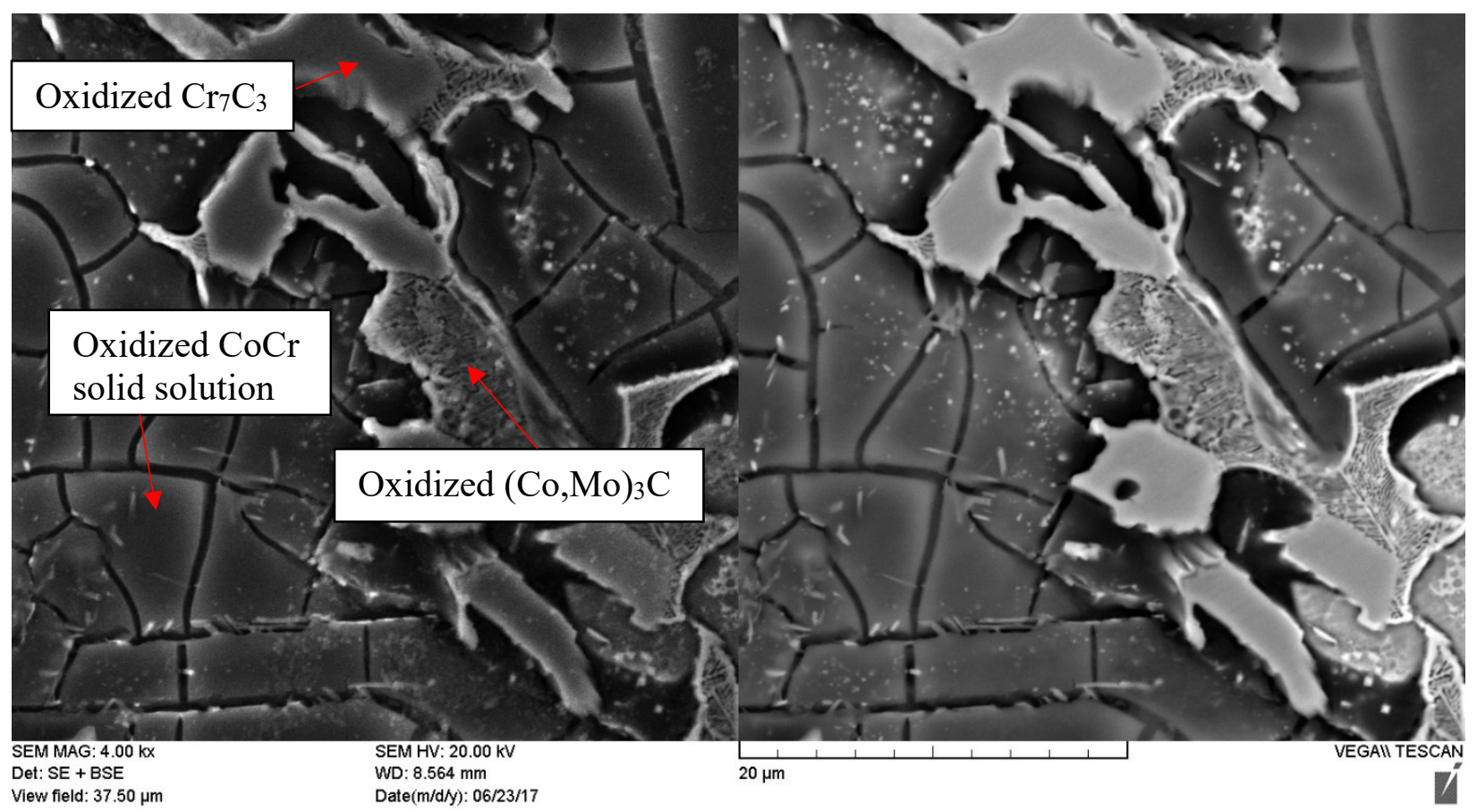

Figure 4-41: 4000x magnification image of Stellite 706 showing fractured oxide layer regions within CoCr solid solution

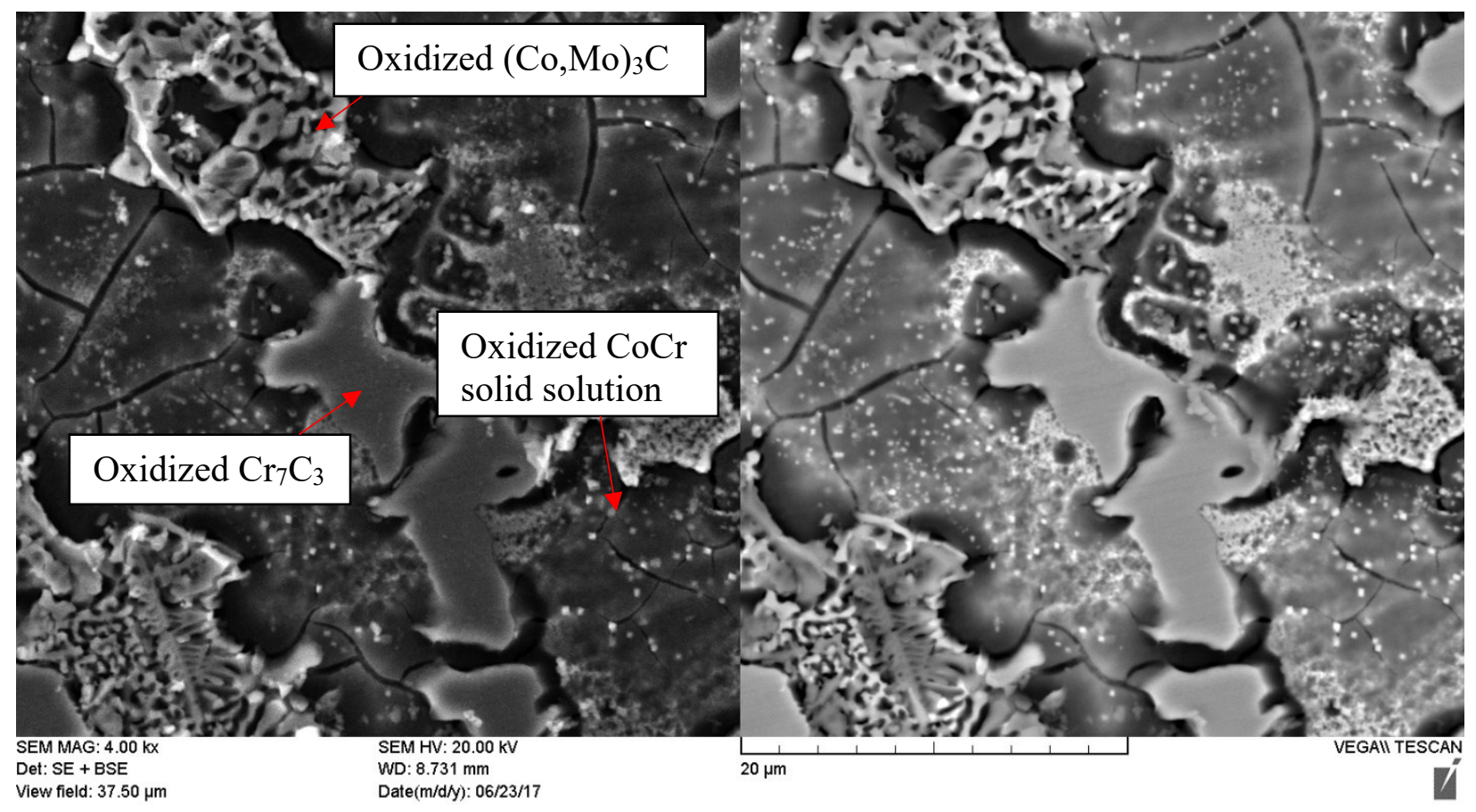

Figure 4-42: 4000x magnification image of Stellite 712 showing fractured oxide layer regions within CoCr solid solution 


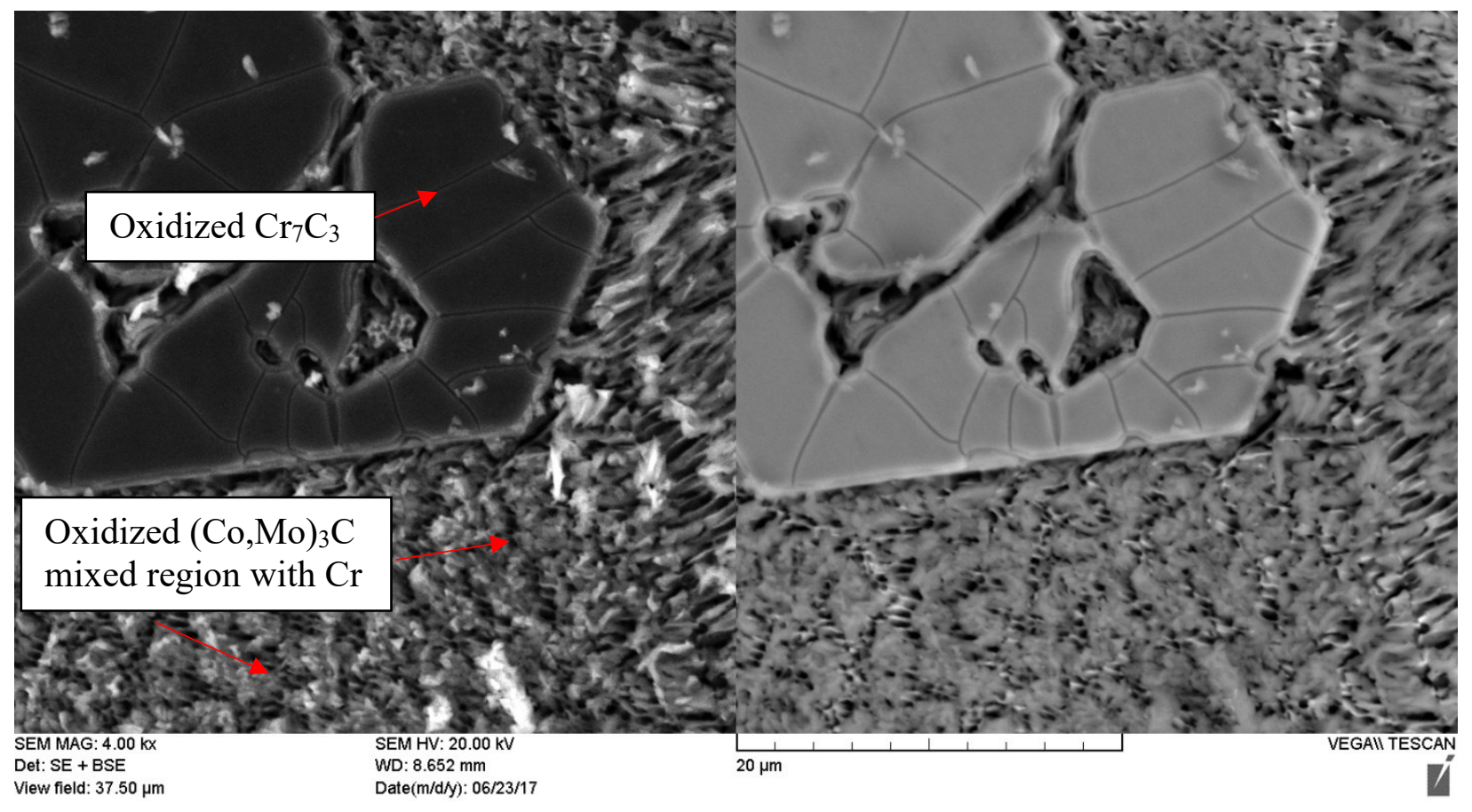

Figure 4-43: 4000x magnification image of Stellite 720 showing fractured oxide layer region on $\mathrm{Cr}_{7} \mathrm{C}_{3}$ surface

To further confirm if the surface is oxidized, EDS analysis was performed on the corroded surfaces. It should be noted that although $\mathrm{C}$ is an essential addition to 700 series Stellite alloys, the EDS system cannot accurately detect the compositions of nonmetallic elements such as $\mathrm{C}$ and $\mathrm{O}$, hence the EDS results with these elements can only be qualitative but not quantitative. Figure 4-44 shows dark regions in Stellite 706 that has numerous fractured oxide layers, which was detected to have high $\mathrm{O}$ and $\mathrm{Cr}$ contents reported in Table 4-5. The reason for the fractures could pertain to the increasing potential value during cyclic polarization test. The light region contains very low $\mathrm{O}$ with high Cr contents, which denotes Cr carbides. Compared with the intact surface of Stellite 706 in Figure 4-38(b), the black region in the microstructure is $\mathrm{Cr}$ carbides, which corresponds to the light region of the corroded surface. The $\mathrm{Cr}$ carbide phase can be oxidized during the corrosion process, however, the $\mathrm{O}$ concentration at this region is low and the majority of the oxide is formed 
with solute $\mathrm{Cr}$ in the solid solution and $\mathrm{Mo}$ within the $(\mathrm{Co}, \mathrm{Mo})_{3} \mathrm{C}$ intermetallic region as indicated from Table 4-5 to Table 4-7.

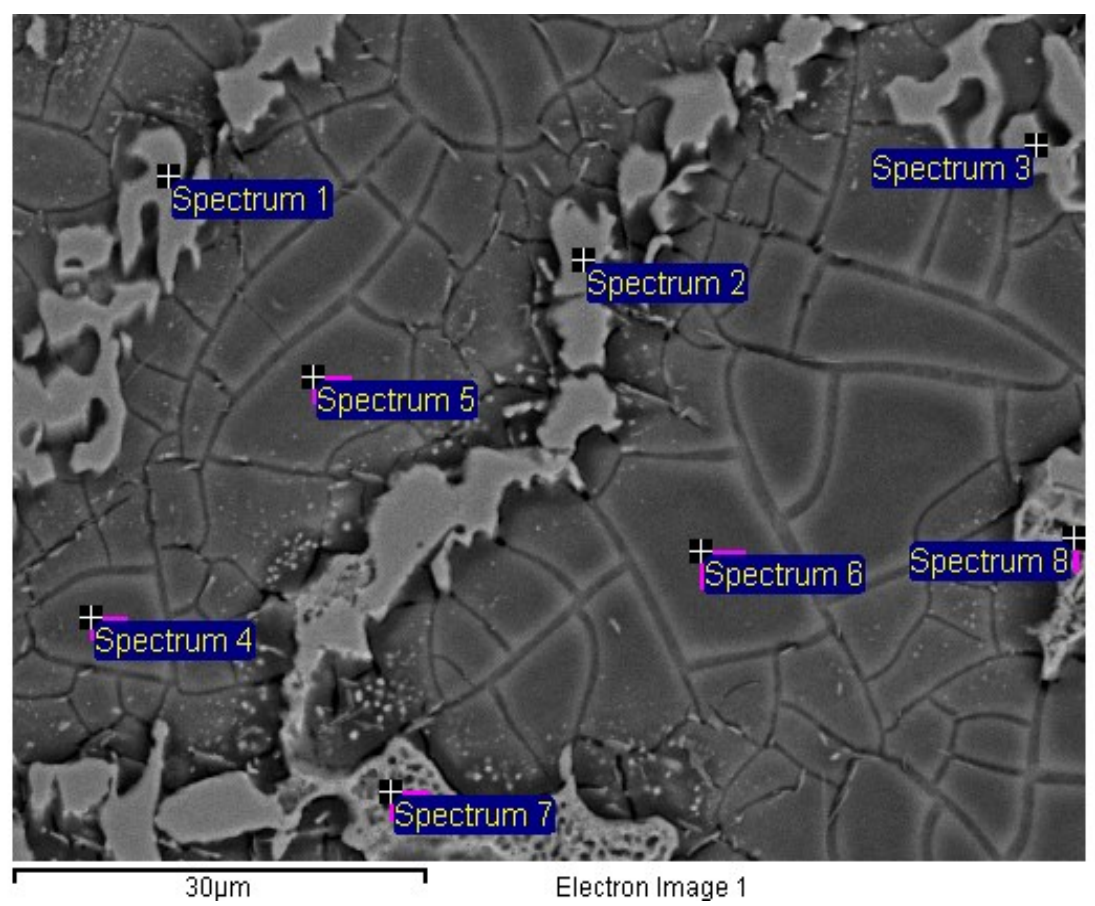

Figure 4-44: Stellite 706 surface after polarization test

Table 4-5: EDS elemental concentration (wt.\%) of corroded Stellite 706 surface

\begin{tabular}{|c|c|c|c|c|c|c|c|c|c|c|}
\hline Spectrum & C & O & Co & Cr & Mo & Fe & Ni & Mn & Si & Total \\
\hline Spectrum 1 & 21.80 & 6.15 & 12.05 & 82.55 & 5.79 & 0.98 & 0.47 & 0.81 & 0.03 & 130.62 \\
\hline Spectrum 2 & 18.26 & 3.86 & 12.30 & 81.28 & & & & & & 115.70 \\
\hline Spectrum 3 & 24.26 & 4.29 & 11.79 & 79.91 & 5.09 & 0.86 & & & & 126.21 \\
\hline Spectrum 4 & 24.10 & 32.06 & 50.17 & 31.22 & 2.76 & 6.00 & 1.87 & 0.86 & 3.95 & 152.98 \\
\hline Spectrum 5 & 24.44 & 33.30 & 52.34 & 30.23 & 2.92 & 5.65 & & & 3.96 & 152.84 \\
\hline Spectrum 6 & 22.61 & 32.77 & 49.23 & 29.86 & 2.42 & 5.80 & & & 4.00 & 146.70 \\
\hline Spectrum 7 & 16.29 & 27.12 & 26.42 & 31.01 & 29.52 & 2.49 & 0.83 & 1.03 & 3.32 & 138.02 \\
\hline Spectrum 8 & 18.22 & 25.52 & 22.71 & 34.04 & 24.74 & 2.50 & & 1.15 & 3.47 & 132.36 \\
\hline
\end{tabular}

Stellite 712 in Figure 4-45 display similar results compared to Stellite 706. The smoother region (spectrum 1 and spectrum 2) contains low $\mathrm{O}$ but high $\mathrm{Cr}$ as indicated in Table 4-6, which are the $\mathrm{Cr}$ carbide regions. The fractured regions (spectrum 3 and spectrum 4) and the whiter regions 
(spectrum 5 and spectrum 6) contain high concentrations of $\mathrm{O}$, which indicates once again that oxide primarily resides within $\mathrm{CoCr}$ solid solution and $(\mathrm{Co}, \mathrm{Mo}){ }_{3} \mathrm{C}$ intermetallic regions.

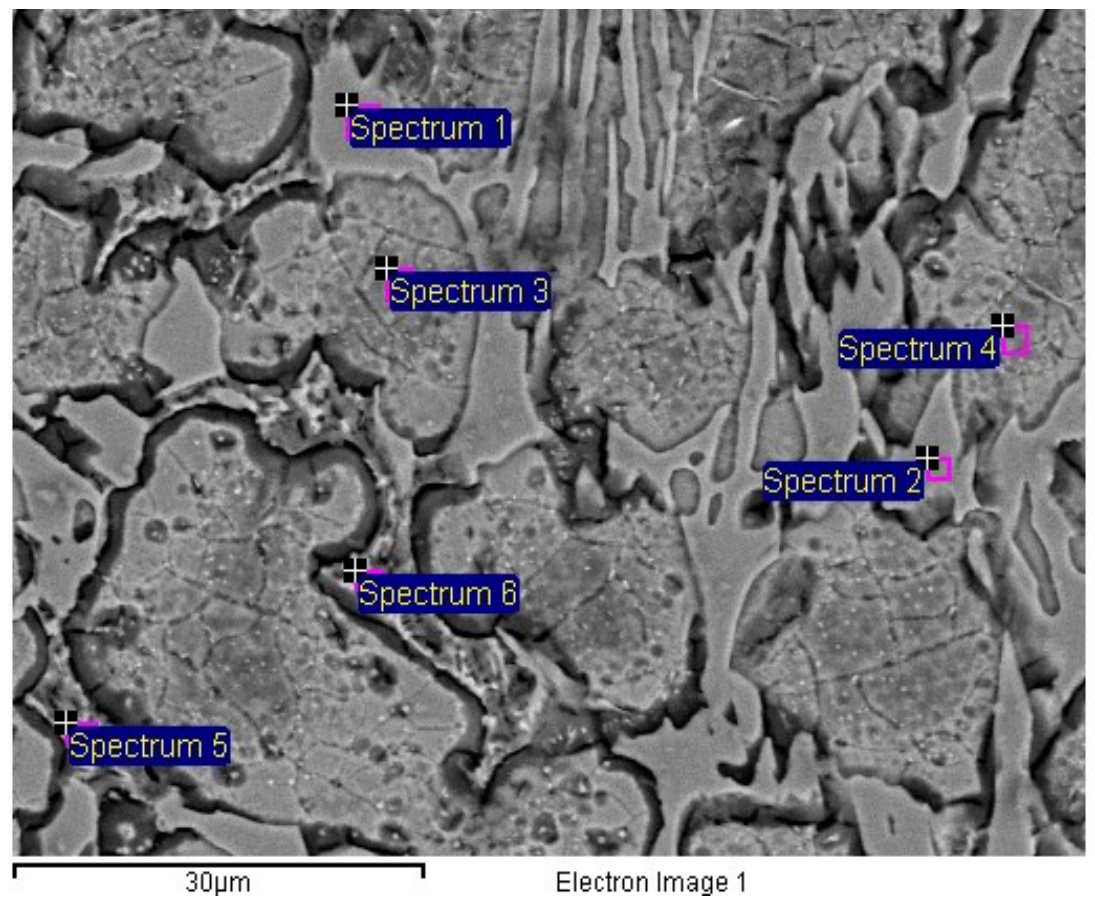

Figure 4-45: Stellite 712 surface after polarization test

Table 4-6: EDS elemental concentration (wt.\%) of corroded Stellite 712 surface

\begin{tabular}{|c|c|c|c|c|c|c|c|c|c|c|}
\hline Spectrum & C & O & Co & Cr & Mo & Fe & Ni & Mn & Si & Total \\
\hline Spectrum 1 & 19.62 & 12.89 & 16.65 & 76.59 & 11.21 & 1.48 & 0.38 & 0.72 & 0.08 & 139.62 \\
\hline Spectrum 2 & 16.06 & 10.97 & 14.85 & 77.29 & & 1.16 & & & & 120.33 \\
\hline Spectrum 3 & 17.16 & 27.08 & 61.92 & 28.67 & 6.73 & 7.18 & & & 1.40 & 150.14 \\
\hline Spectrum 4 & 18.91 & 24.44 & 63.07 & 27.53 & 6.34 & 7.53 & & & 0.72 & 148.54 \\
\hline Spectrum 5 & 21.87 & 35.61 & 18.03 & 42.30 & 22.44 & 4.78 & & & 0.76 & 145.80 \\
\hline Spectrum 6 & 18.09 & 24.65 & 16.36 & 46.31 & 19.73 & 4.41 & & & 0.60 & 130.14 \\
\hline
\end{tabular}

The morphology of corroded Stellite 720 surface looks quite different from that of Stellite 706 and Stellite 712. As seen in Figure 4-46, due to high C and Mo content, the matrix is now a mixture of Co, $\mathrm{Cr}$ and Mo. EDS results in Table 4-7 show that the large second phase particle (spectrum 1) is 
$\mathrm{Cr}$ carbide since it contains small $\mathrm{O}$ concentration. The rest of the surrounding matrix have higher O concentration, indicating the presence of oxides. However, it should be noted that according to the microstructure of Stellite 720, shown in Figure 4-40(b), the oxide layer must be discrete and is small in size than that of the other two alloys, because of the large amount of carbides embedded in the matrix, hence they are too small to see in detail in the SEM images.

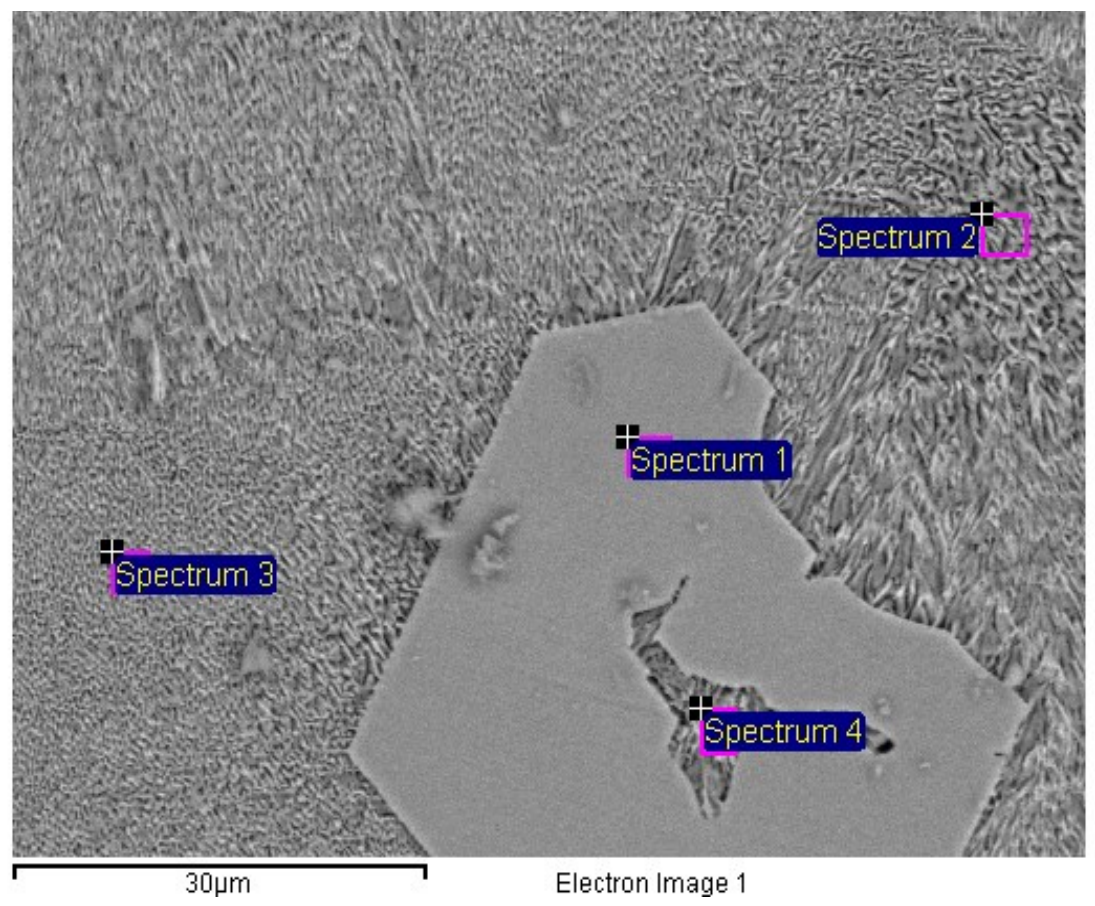

Figure 4-46: Stellite 720 surface after polarization test

Table 4-7: EDS elemental concentration (wt.\%) of corroded Stellite 720 surface

\begin{tabular}{|c|c|c|c|c|c|c|c|c|c|c|}
\hline Spectrum & C & O & Co & Cr & Mo & Fe & Ni & Mn & Si & Total \\
\hline Spectrum 1 & 15.78 & 6.09 & 9.05 & 80.43 & 12.51 & 0.87 & 0.01 & 1.28 & 0.14 & 126.16 \\
\hline Spectrum 2 & 17.56 & 24.82 & 25.10 & 41.89 & 25.18 & 3.67 & & & 1.27 & 139.50 \\
\hline Spectrum 3 & 19.38 & 20.78 & 26.81 & 41.57 & 28.32 & 3.77 & & & 1.31 & 141.94 \\
\hline Spectrum 4 & 20.27 & 22.31 & 29.94 & 43.28 & 23.54 & 3.44 & & & 1.24 & 144.03 \\
\hline
\end{tabular}


To further provide clarity, Figure 4-47 shows the EDS spectra of Stellite 706 of spectrums 1, 4 and 7 chosen as examples to show that each region has different peaks that are analogous to the EDS results shown in Table 4-5.

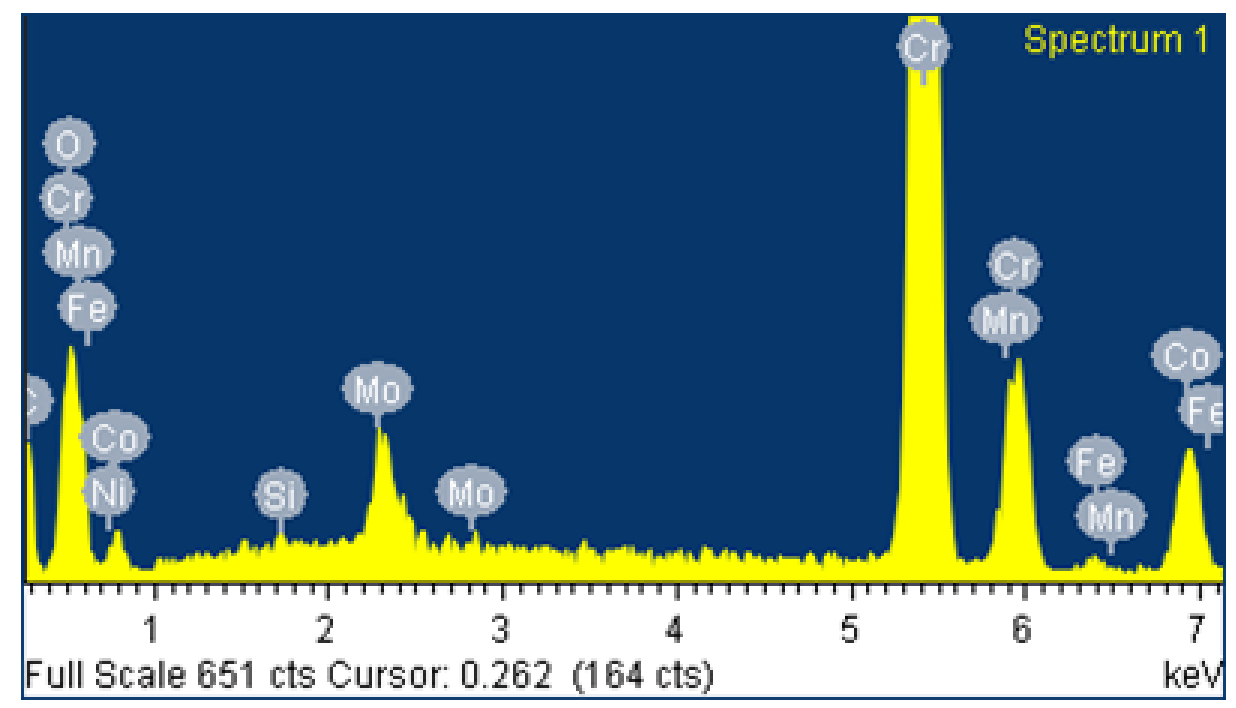

(a)

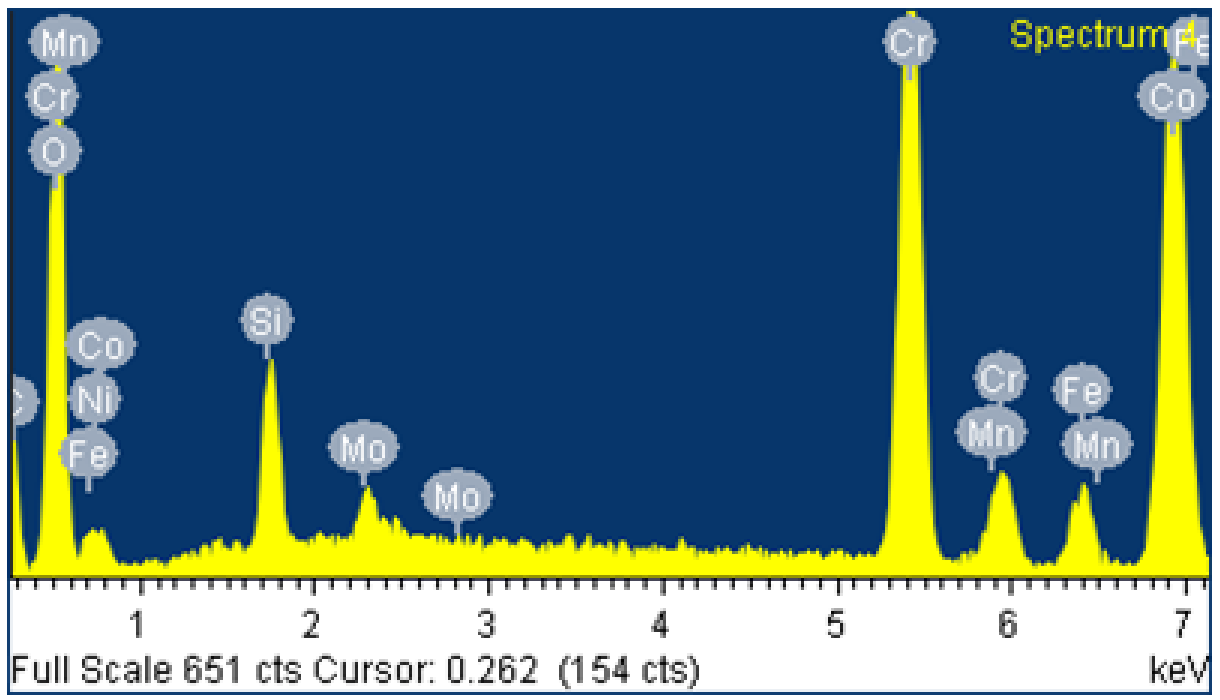

(b) 


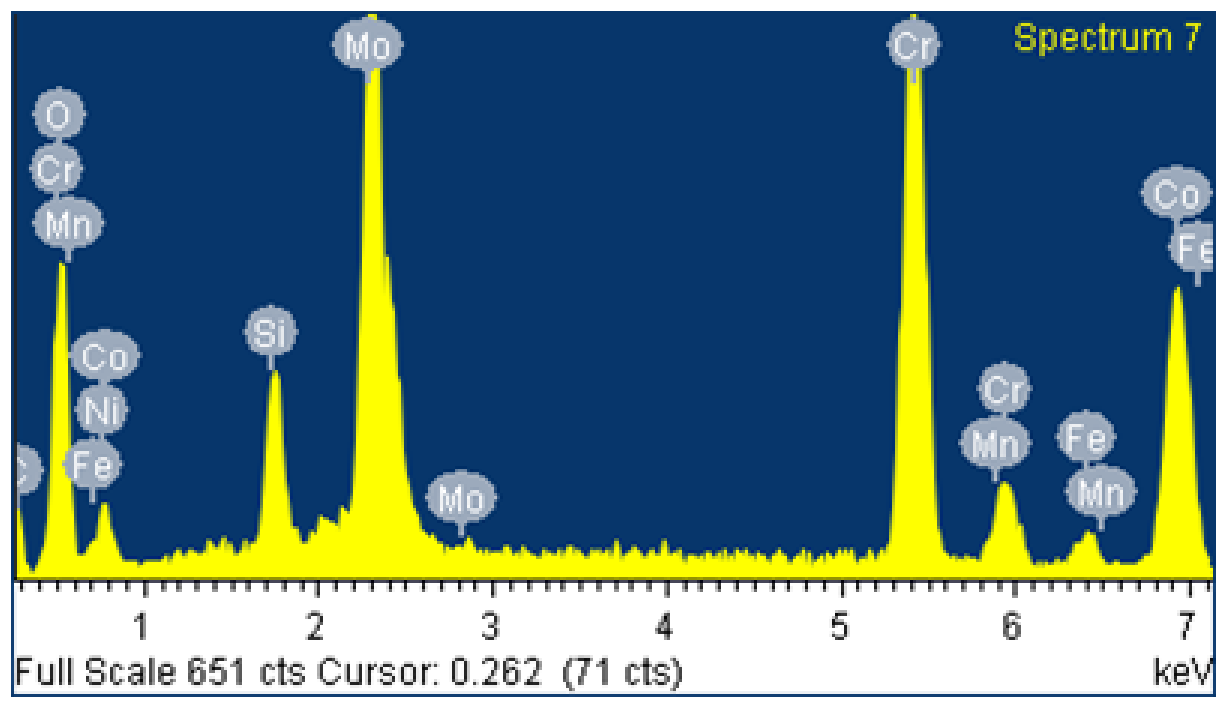

(c)

Figure 4-47: EDS spectra of Stellite 706: (a) Cr-rich region, (b) $\mathrm{Cr}$ and Co-rich region and (c) Mo-rich region

\subsection{Failure Tests}

\subsubsection{Potentiostatic polarization}

From EIS and potentiostatic tests, Mo influences corrosion performance as Stellite 720 comparatively shows the best performance. To test the alloy limitations, potentiostatic polarization can be used to assess an alloys current behaviour at a specified constant potential. When holding a voltage constant for a set period time, the alloy tested can eventually come to a stable region in current behaviour. In this case, if Stellite 720 displays the lowest stable current behaviour in comparison, it can further confirm the effects of Mo. The tests were done in morpholine solution with $\mathrm{pH} 9.5$ after 1.5 hours $\mathrm{OCP}$ at a constant temperature of $25^{\circ} \mathrm{C}$ and voltages of $1 \mathrm{~V}, 2 \mathrm{~V}$ and 3 $\mathrm{V}$, respectively, on the alloy's surface for one hour. The obtained polarization curves are shown in Figure 4-48 to Figure 4-50 for one out of the three experiments done for each alloy. 


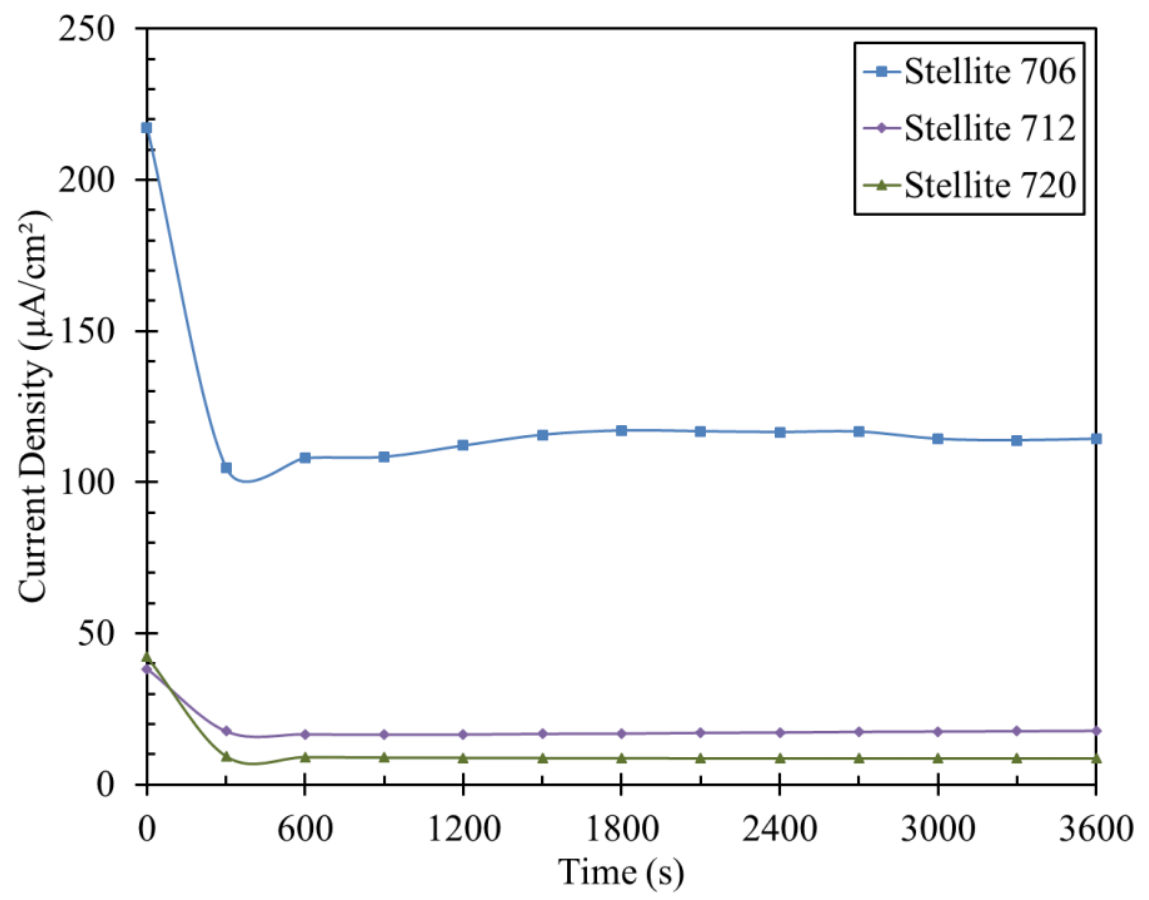

Figure 4-48: Current density variation for $1 \mathrm{~V}$ potential

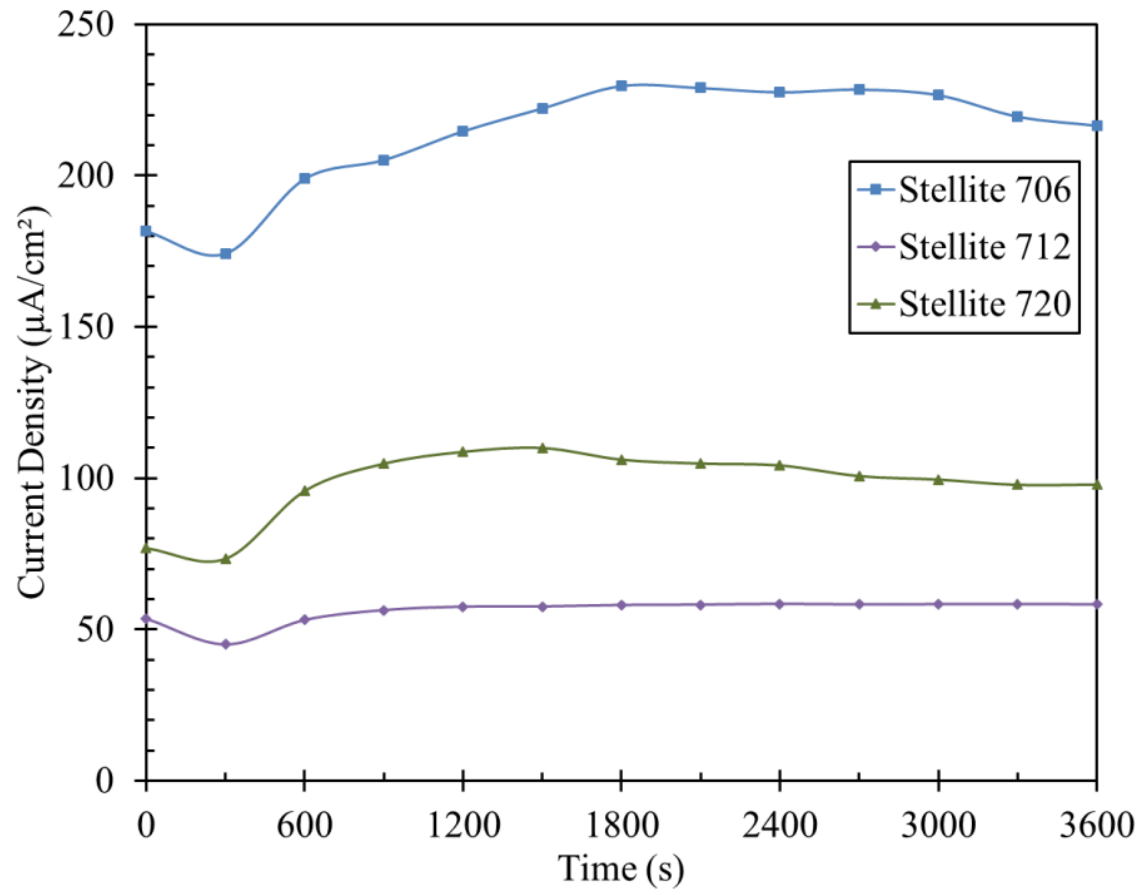

Figure 4-49: Current density variation for $2 \mathrm{~V}$ potential 


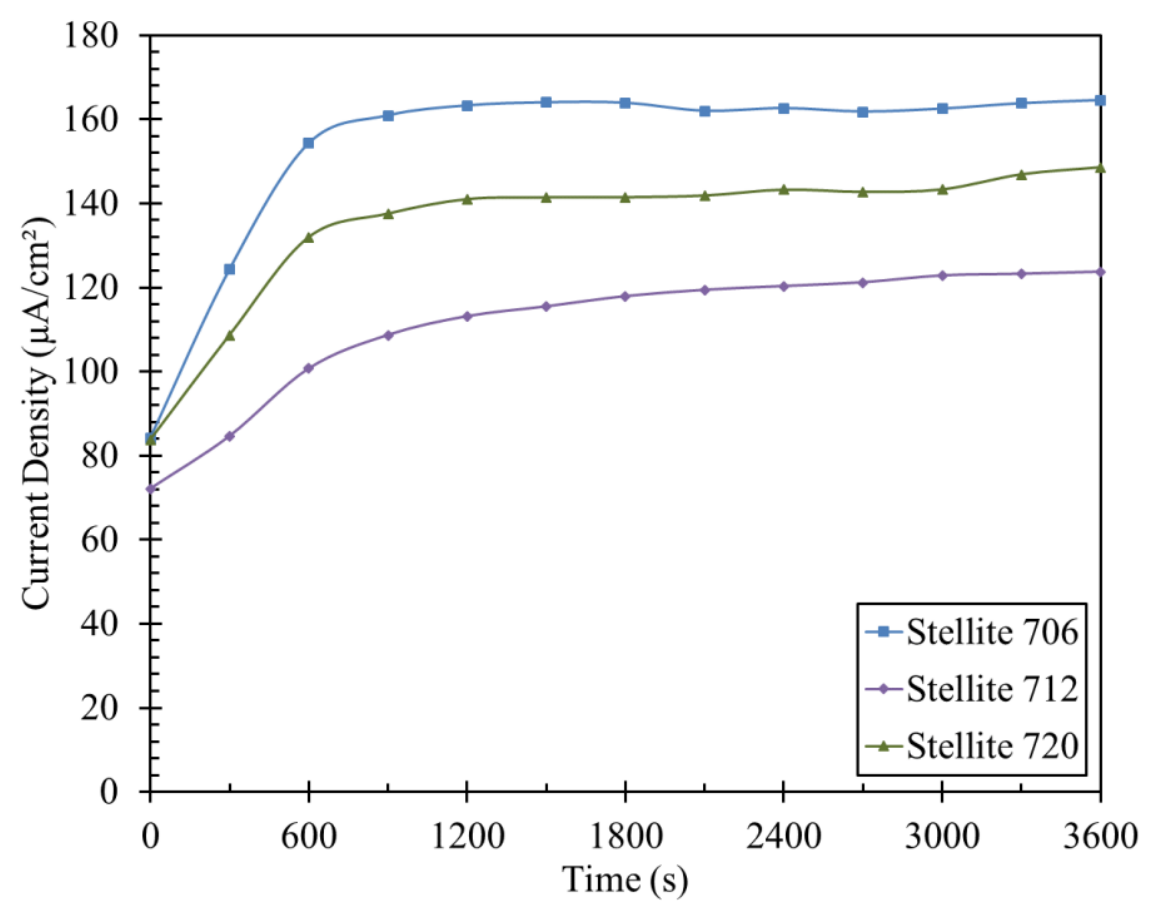

Figure 4-50: Current density variation for $3 \mathrm{~V}$ potential

For potentiostatic testing, the current density at some point of time within the duration of test displays a stable behaviour, as shown from the more linear horizontal lines. At this stable region, the current density of the alloy can be taken as the corrosion current density $\left(I_{\text {corr }}\right)$ for the voltage applied. In all cases of applied constant potential $(1 \mathrm{~V}, 2 \mathrm{~V}$ and $3 \mathrm{~V})$, Stellite 706 has the largest current density, indicating that its corrosion resistance is poor in comparison to Stellite 712 and Stellite 720. Between Stellite 712 and Stellite 720, the difference is not significant, but in general, Stellite 712 is better. However, the results here in comparison to previous EIS and cyclic polarization tests do not match. With Stellite 720 having the most Mo content, it was expected that it would have the lowest current density value, however, that was not the case. 


\subsubsection{Corrosion rate}

It is normally assumed that the oxidation process is uniform over the alloy surface and oxidation is not selectively occurring on a single element. Thus, the equivalent weight (EW) expression from Eq (5) normally considers a unit mass of the alloy oxidized. Given the composition of 700 Stellite alloys, the first likely oxidation reaction to occur will be with $\mathrm{Cr}$ as noted from the Standard Electrode Potential table [45]. In general, elements above 1 mass percent is considered in the EW calculations, however in the cases where the exact composition is not given for certain elements of the alloy a mid-range of the composition can be used. For all the three 700 Stellite alloys, the exact compositions of $\mathrm{Ni}, \mathrm{Fe}, \mathrm{Si}$, and $\mathrm{Mn}$ are unknown, but the maximum and minimum composition are known. Thus, for calculation simplicity, $2 \%$ by mass was used for $\mathrm{Fe}$ and $\mathrm{Ni}$ and $1.25 \%$ by mass was used for Si and Mn.

The valence values of each metal were obtained from individual Pourbaix diagrams [38] in a water system, which are summarized in Table 4-8, showing the stable phases of each element at $E_{\text {corr }}$ in $\mathrm{pH}$ 9.5. The density of each alloy was calculated, and the results are given in Table 4-9, with an implicit assumption that the alloy specimens were isotropic. After weighing each specimen before and after the potentiostatic tests, the time span of one hour only changed the weight of each alloy by the fourth decimal place. Due to extremely low mass loss, the resulting corrosion rate and the mass loss rate are extremely low. By comparison alone, the values in Table 4-10 indicate that Stellite 712 performed better only at $2 \mathrm{~V}$ and $3 \mathrm{~V}$ potentials, while Stellite 720 performed better at $1 \mathrm{~V}$. However, the error values for $I_{\text {corr }}$ are extremely large making it difficult to evaluate the performance of each alloy accurately. 
Table 4-8: Valence values used in EW calculations

\begin{tabular}{|c|c|c|c|c|c|c|c|c|}
\hline Elements & $\mathbf{C o}$ & $\mathbf{C r}$ & $\mathbf{M o}$ & $\mathbf{C}$ & $\mathbf{M n}$ & $\mathbf{F e}$ & $\mathbf{S i}$ & $\mathbf{N i}$ \\
\hline $\begin{array}{c}\text { Reaction at 9.5 pH at } \\
\text { Ecorr }\end{array}$ & $\mathrm{Co}(\mathrm{OH})_{2}$ & $\mathrm{Cr}_{2} \mathrm{O}_{3}$ & $\mathrm{MoO}_{4}{ }^{2-}$ & $\mathrm{HCO}_{3}{ }^{-}$ & $\mathrm{Mn}^{2+}$ & $\mathrm{FeOH}^{+}$ & $\mathrm{H}_{4} \mathrm{SiO}_{4}$ & $\mathrm{Ni}^{2+}$ \\
\hline Valence & $2+$ & $3+$ & $6+$ & $4+$ & $2+$ & $2+$ & $4+$ & $2+$ \\
\hline
\end{tabular}

Table 4-9: Density and EW values

\begin{tabular}{|c|c|c|c|}
\hline Alloy & Stellite 706 & Stellite 712 & Stellite 720 \\
\hline Mass (gram) & 2.66 & 2.60 & 2.69 \\
\hline Density $\left(\boldsymbol{g} / \mathbf{c m}^{\mathbf{3}}\right)$ & 8.28 & 8.07 & 8.36 \\
\hline EW (dimensionless) & $4.23 \mathrm{E}-5$ & $3.95 \mathrm{E}-5$ & $3.58 \mathrm{E}-5$ \\
\hline
\end{tabular}

Table 4-10: Potentiostatic polarization results under constant potentials at $25^{\circ} \mathrm{C}$

\begin{tabular}{|c|c|c|c|c|}
\hline Voltage & Alloy & $I_{\text {corr }}\left(\mathbf{n A} / \mathbf{c m}^{2}\right)$ & CR $(\mathbf{n m} / \mathbf{y r})$ & MR $\left(\boldsymbol{\mu g} / \mathbf{d m} \mathbf{m}^{\mathbf{2}} \mathbf{)}\right.$ \\
\hline \multirow{3}{*}{$1 \mathrm{~V}$} & Stellite 706 & $70.7 \pm 47.2$ & 1.18 & 0.27 \\
\cline { 2 - 5 } & Stellite 712 & $31.2 \pm 22.1$ & 0.50 & 0.11 \\
\cline { 2 - 5 } & Stellite 720 & $14.5 \pm 5.2$ & 0.20 & 0.05 \\
\hline \multirow{3}{*}{$2 \mathrm{~V}$} & Stellite 706 & $167.3 \pm 108.3$ & 2.79 & 0.63 \\
\cline { 2 - 5 } & Stellite 712 & $66.3 \pm 8.7$ & 1.06 & 0.23 \\
\cline { 2 - 5 } & Stellite 720 & $88.4 \pm 21.9$ & 1.24 & 0.28 \\
\hline \multirow{3}{*}{$3 \mathrm{~V}$} & Stellite 706 & $291.7 \pm 230.6$ & 4.87 & 1.10 \\
\cline { 2 - 5 } & Stellite 712 & $122.3 \pm 8.9$ & 1.96 & 0.43 \\
\cline { 2 - 5 } & Stellite 720 & $157.4 \pm 20.1$ & 2.20 & 0.50 \\
\hline
\end{tabular}

Furthermore, an example current density curve is shown in Figure $4-51$ for a $3 \mathrm{~V}$ test in $50^{\circ} \mathrm{C}$ showing extreme random behaviour. It is not practical to approximate an $I_{\text {corr }}$ value with such variation of values. In this condition, even with longer time, the stability of the current density may not be reached. Thus, the average value of $I_{\text {corr }}$ cannot be obtained directly on the current density curve with such large deviations. 


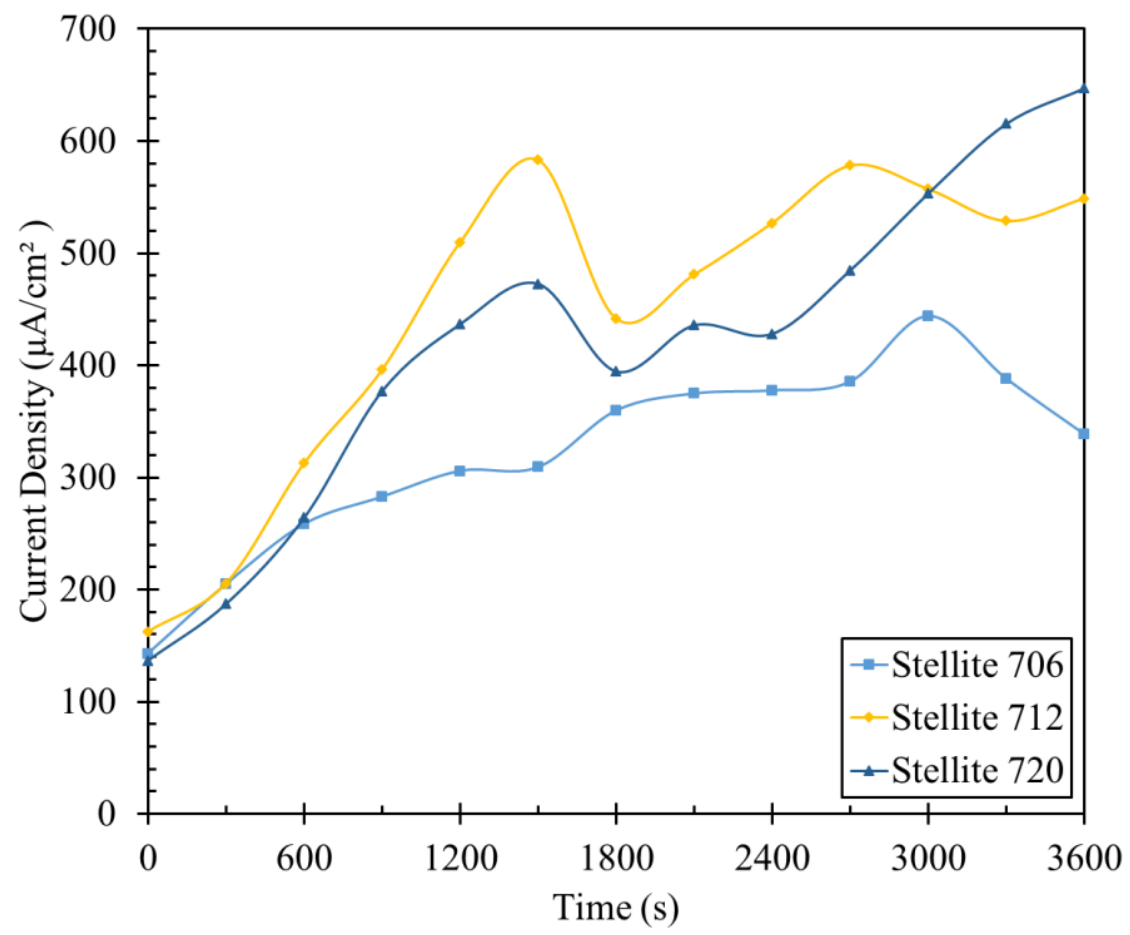

Figure 4-51: Current variation for the condition of $3 \mathrm{~V}$ potential at $50^{\circ} \mathrm{C}$

\subsubsection{Overall current}

A more reasonable approach in quantifying the graphs of current density for each voltage would be to calculate the area under the curve utilizing each set of data points collected. A numerical analysis method called the trapezoidal rule [46] can be used, which is a technique used for approximating the definite integral. Since each graph previously shown has a random output value per point which is independent of its preceding points, the representation of using an average is not ideal because the initial portion of every graph is essentially a slope. A graphical representation of the trapezoid rule is shown in Figure 4-52. It illustrates a simple process of how each point on a curve can be represented as a form of a trapezoid to calculate the area under the curve. As a result, the graphs generated from the potentiostatic tests can be simplified by straight lines connecting with each point from start to finish, thus the area is the sum of every trapezoid section. 


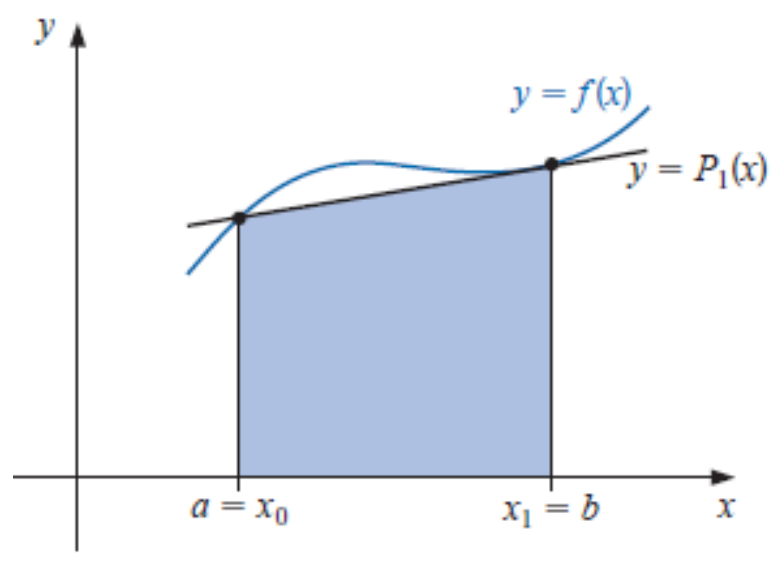

Figure 4-52: Trapezoid rule plot [46]

The equation used to calculate the area under the curve is as follows [46]:

$$
\int_{a}^{b} f(x) d x \approx \frac{\Delta x}{2} \sum_{i=1}^{N} f\left(x_{i-1}\right)+f\left(x_{i}\right), \Delta x=\frac{b-a}{N}
$$

where $N$ is equally spaced intervals between points $a$ and $b$, point $a$ is $x_{i}$ and point $b$ is $x_{i+1}$. By measuring the area under the curve, a better numerical comparison between the alloys for corrosion performance with respect to current density can be obtained, as presented in Figure 4-53, which shows a comparison of each alloy under $1 \mathrm{~V}$ potential at $25^{\circ} \mathrm{C}$ morpholine solution with $\mathrm{pH} 9.5$. The total one-hour duration was split into six 10-minute intervals to see if each alloy behaved differently in each smaller duration. 


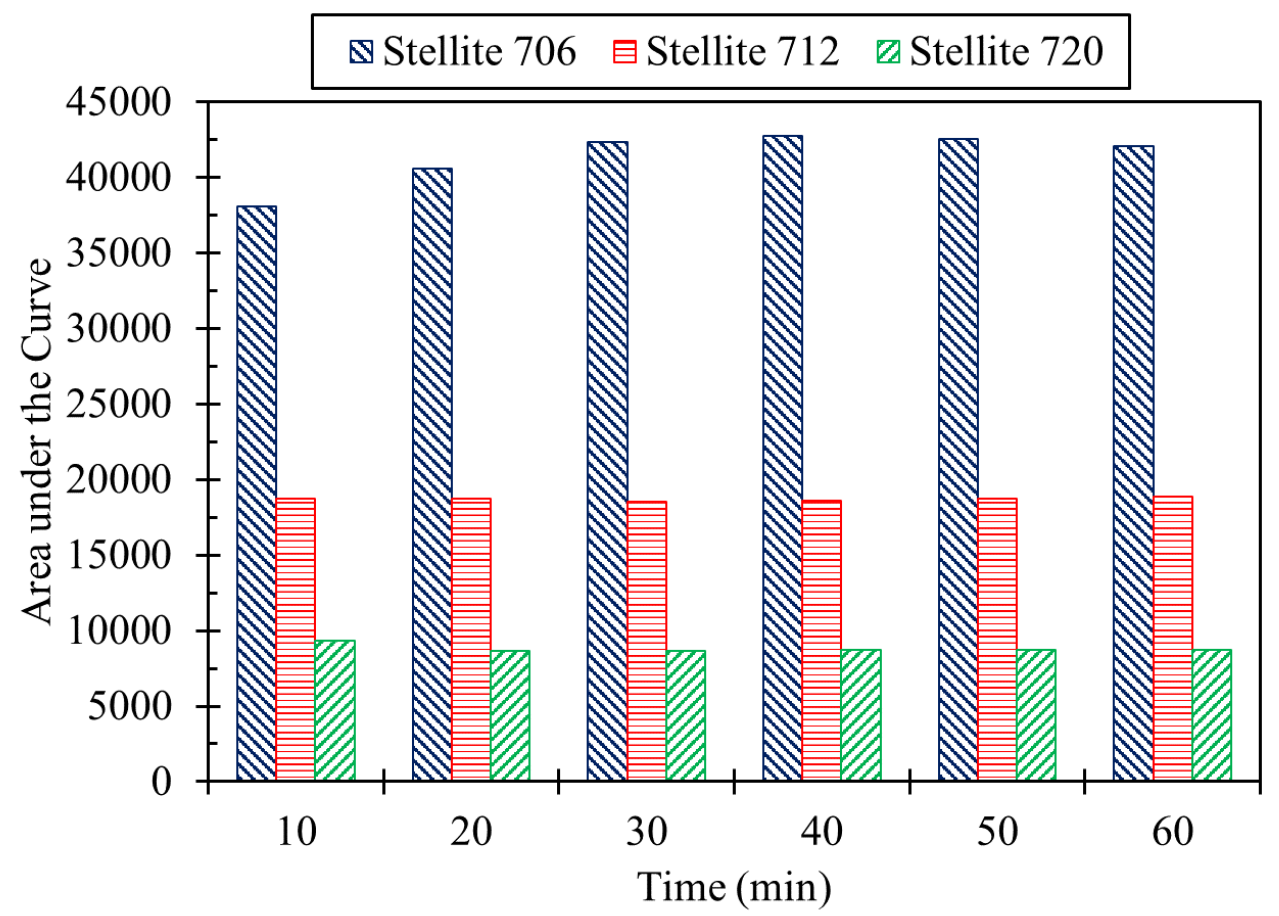

Figure 4-53: Current density variations at $1 \mathrm{~V}$ potential in $25^{\circ} \mathrm{C}$ morpholine solution with $\mathrm{pH} 9.5$

The potentiostatic test results for $1 \mathrm{~V}$ constant potential show no large difference for each individual alloy per 10-minute interval. For $2 \mathrm{~V}$ and $3 \mathrm{~V}$ potential tests, the same trend was observed. The plots for overall current density at the potentials of $1 \mathrm{~V}, 2 \mathrm{~V}$ and $3 \mathrm{~V}$ are given in Figure 4-54. For all voltages, Stellite 712 and Stellite 720 perform better than Stellite 706 at $25^{\circ} \mathrm{C}$ in morpholine, which agrees with the observations in the EIS and cyclic polarization tests. However, in this case, Stellite 720 is not seen as the better performing alloy overall except at $1 \mathrm{~V}$. 


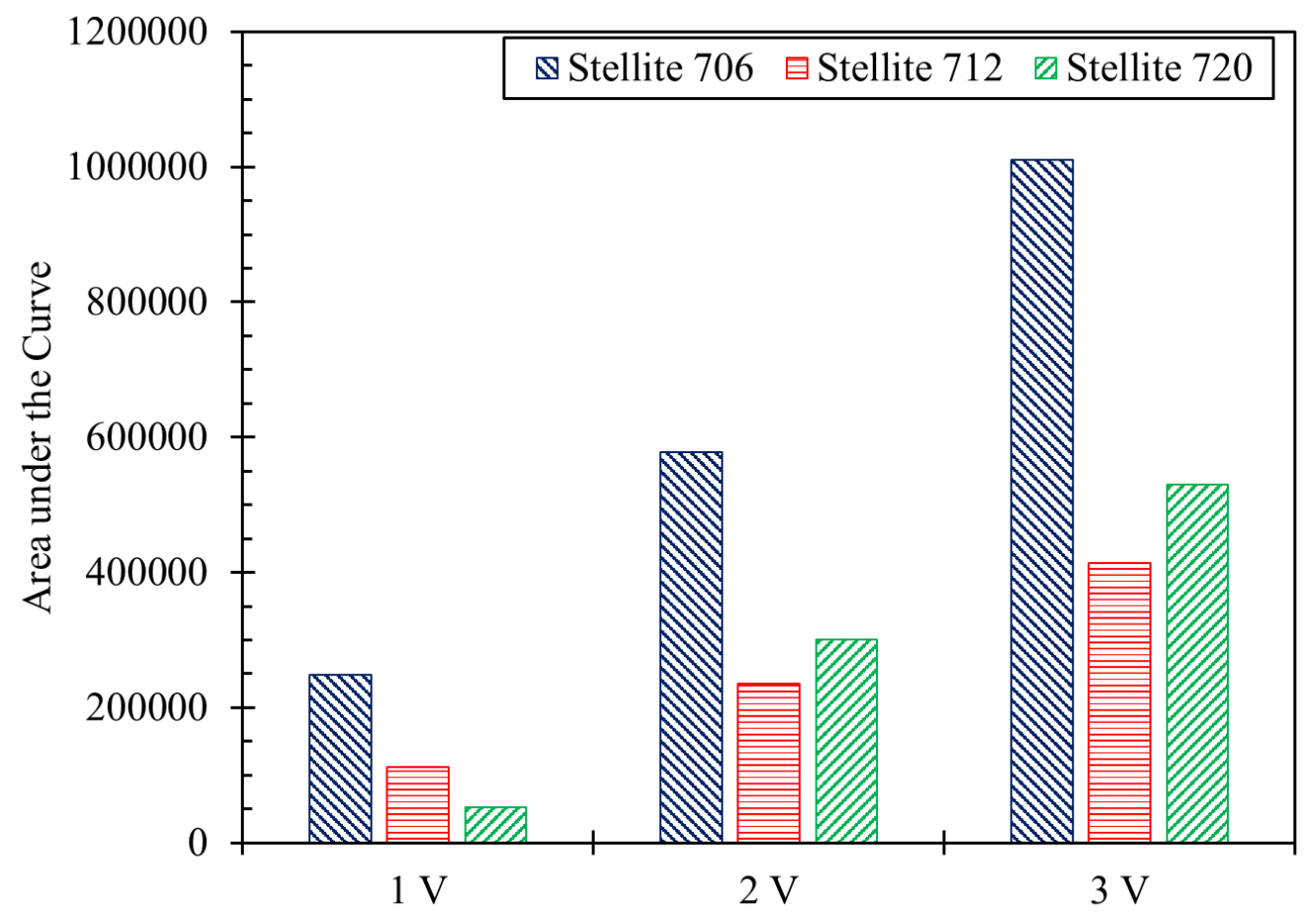

Figure 4-54: Overall current densities at a constant potential in $25^{\circ} \mathrm{C}$ morpholine solution with $\mathrm{pH} 9.5$

For the potentiostatic tests at $50^{\circ} \mathrm{C}$, as shown in Figure 4-55, the three Stellite alloys behaved similarly, particularly, at the potentials of $1 \mathrm{~V}$ and $2 \mathrm{~V}$. In general, temperature enhances chemical reaction due to active atomic motion. According to the current density results for $50^{\circ} \mathrm{C}$, the beneficial effect of Mo might be less pronounced than the detrimental effect of carbides, therefore Stellite 720 and Stellite 706 behaved similarly. In other words, Mo plays a more important role at room temperature than at elevated temperatures for improving the corrosion resistance of Stellite alloys. This can be correlated with the EIS and polarization performance bar graphs shown earlier at $50^{\circ} \mathrm{C}$, where $R_{p}$ in both cases had small changes amongst each alloy. More importantly, the randomness of the potentiostatic tests does not give any information about localized corrosion behaviour when testing the limitations of Stellite alloys. Thus, cyclic polarization at higher potentials is utilized as discussed in the next section. 


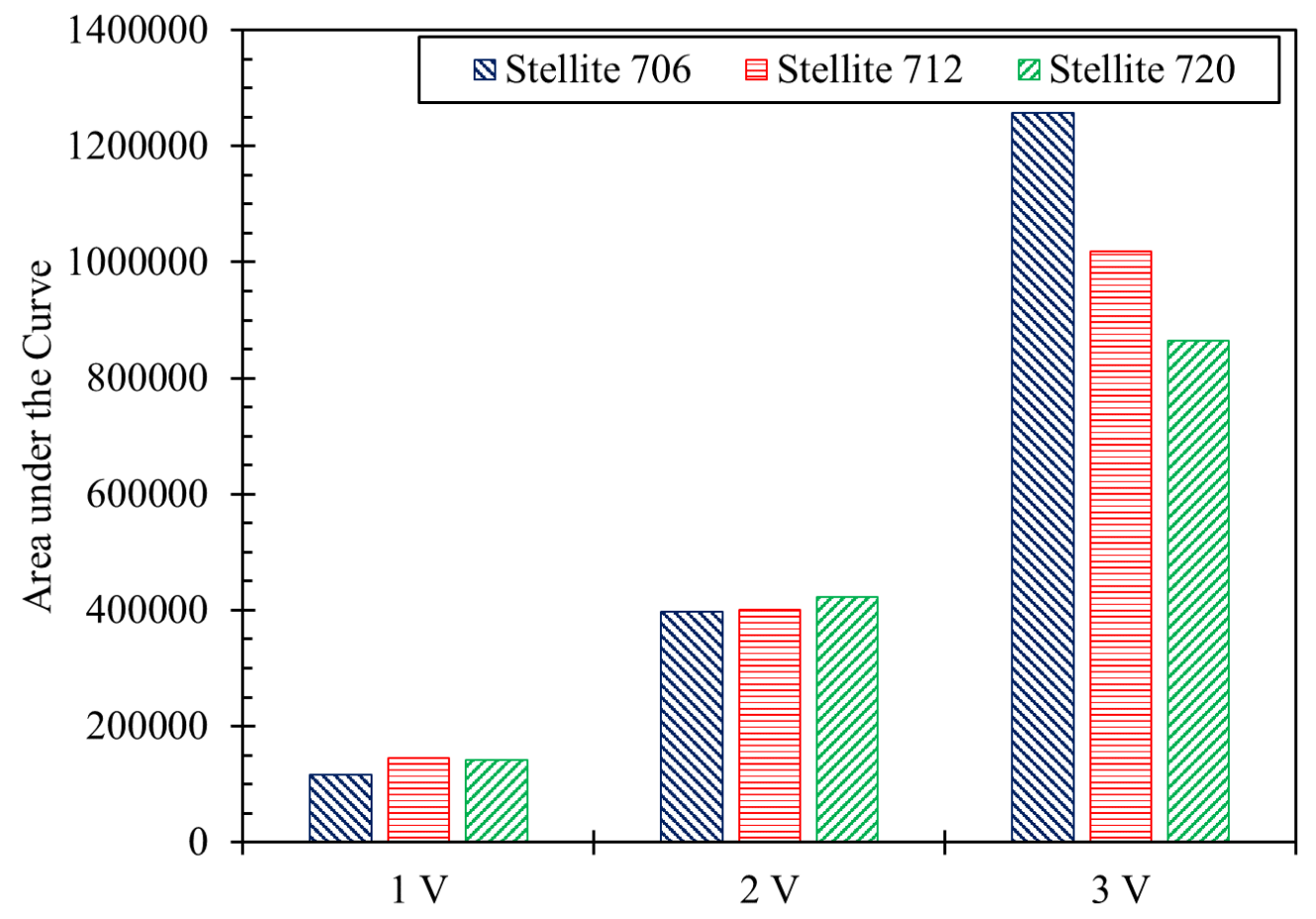

Figure 4-55: Overall current densities at a constant potential in $50^{\circ} \mathrm{C}$ morpholine solution with $\mathrm{pH} 9.5$

\subsubsection{Potentiodynamic polarization}

Since it was difficult to identify performance differences between the three 700 series Stellite alloys at higher potentials using the potentiostatic method, failure polarization test was conducted on these alloys using cyclic polarization. The purpose of this test was to qualitatively determine the maximum voltage that can cause severe oxidation of the alloy surface, that is, visible corrosion, and then observe the alloys oxide stability in the reverse scan. The test was done for peak voltages between $2-12 \mathrm{~V}$ with a higher scan rate at $25 \mathrm{mV} / \mathrm{s}$ under morpholine at $\mathrm{pH} 9.5$. The test was started at $-0.5 \mathrm{~V}$ and taken to $2 \mathrm{~V}$ peak and the peak voltage was increased by one volt for every subsequent test. Qualitatively every test done in different peak voltages displayed the same behaviour for all three alloys, thus only the highest voltage is shown for comparison from Figure 4-56 to Figure 4-58. The spontaneous passivity shown from previous test results for $1.2 \mathrm{~V}$ peak 
voltages is not observed here where all the alloys directly go into transpassive behaviour. All three Stellite alloys had the exact same performance in comparison where none of the alloys went beyond $10^{-3} \mathrm{~A}$ regardless of how high the voltage count was. Compared to $1.2 \mathrm{~V}$ peak tests where the maximum current density was less than $10^{-4} \mathrm{~A}$, the current density for all failure tests in morpholine only goes up by one order of magnitude. None of the alloys displayed signs of severe pitting with voltage increase as the hysteresis displayed a stable behaviour. The surface of each alloy eventually turned dark, which was an indication that oxidation did occur, but the specimens did not appear to degrade severely as displayed in Figure 4-59.

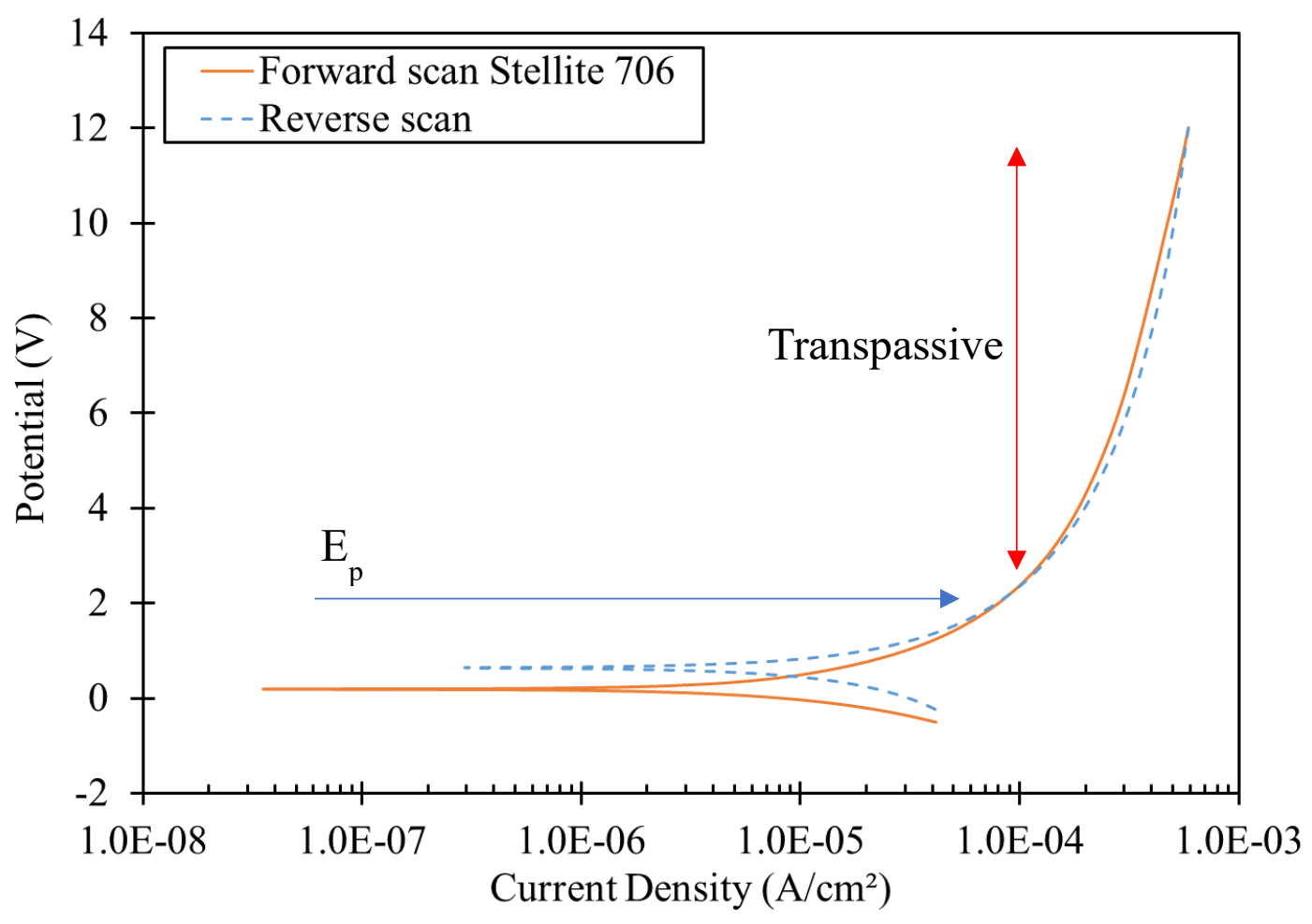

Figure 4-56: Cyclic polarization of Stellite 706 under peak voltage of $12 \mathrm{~V}$ in morpholine 


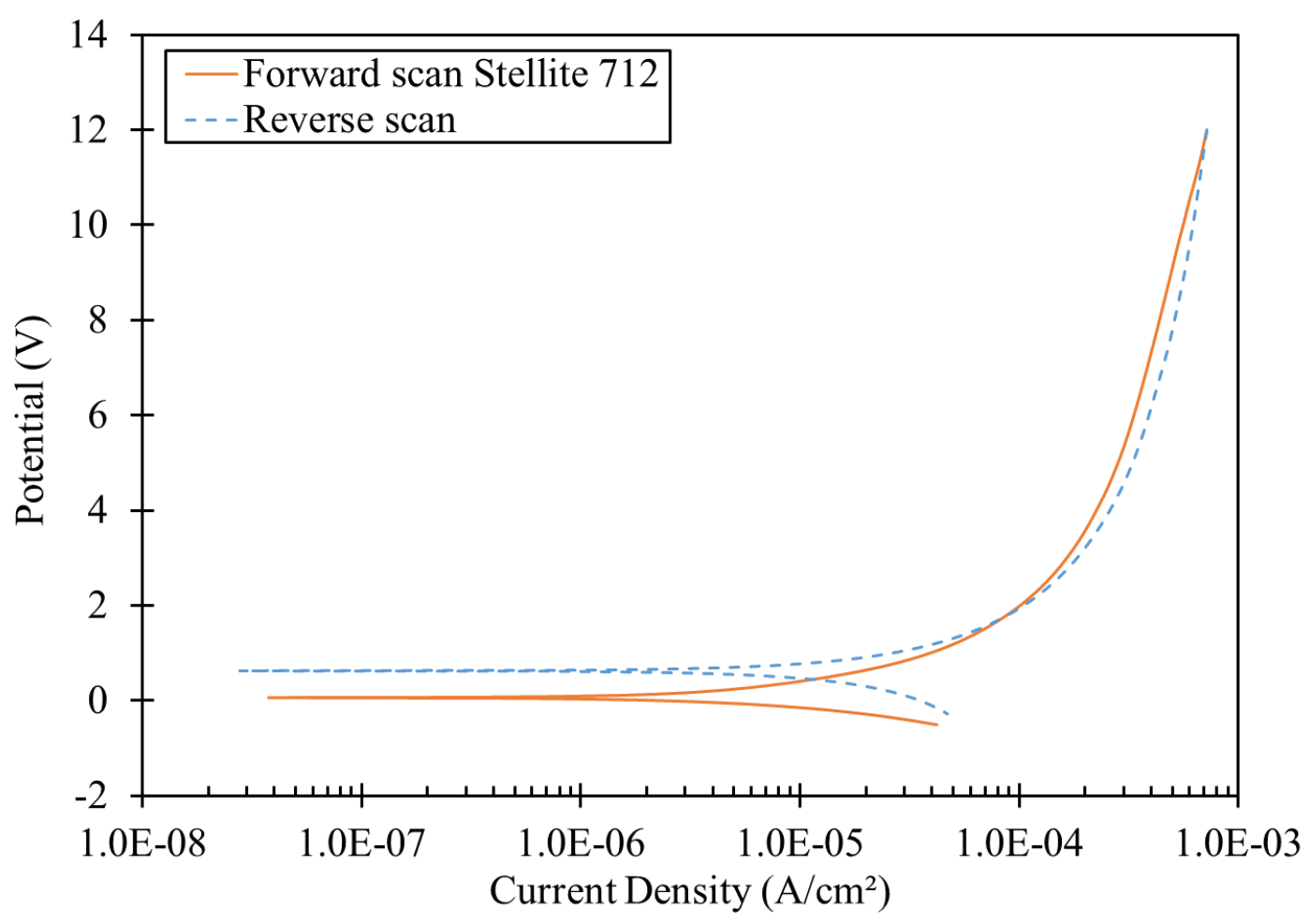

Figure 4-57: Cyclic polarization of Stellite 712 under peak voltage of $12 \mathrm{~V}$ in morpholine

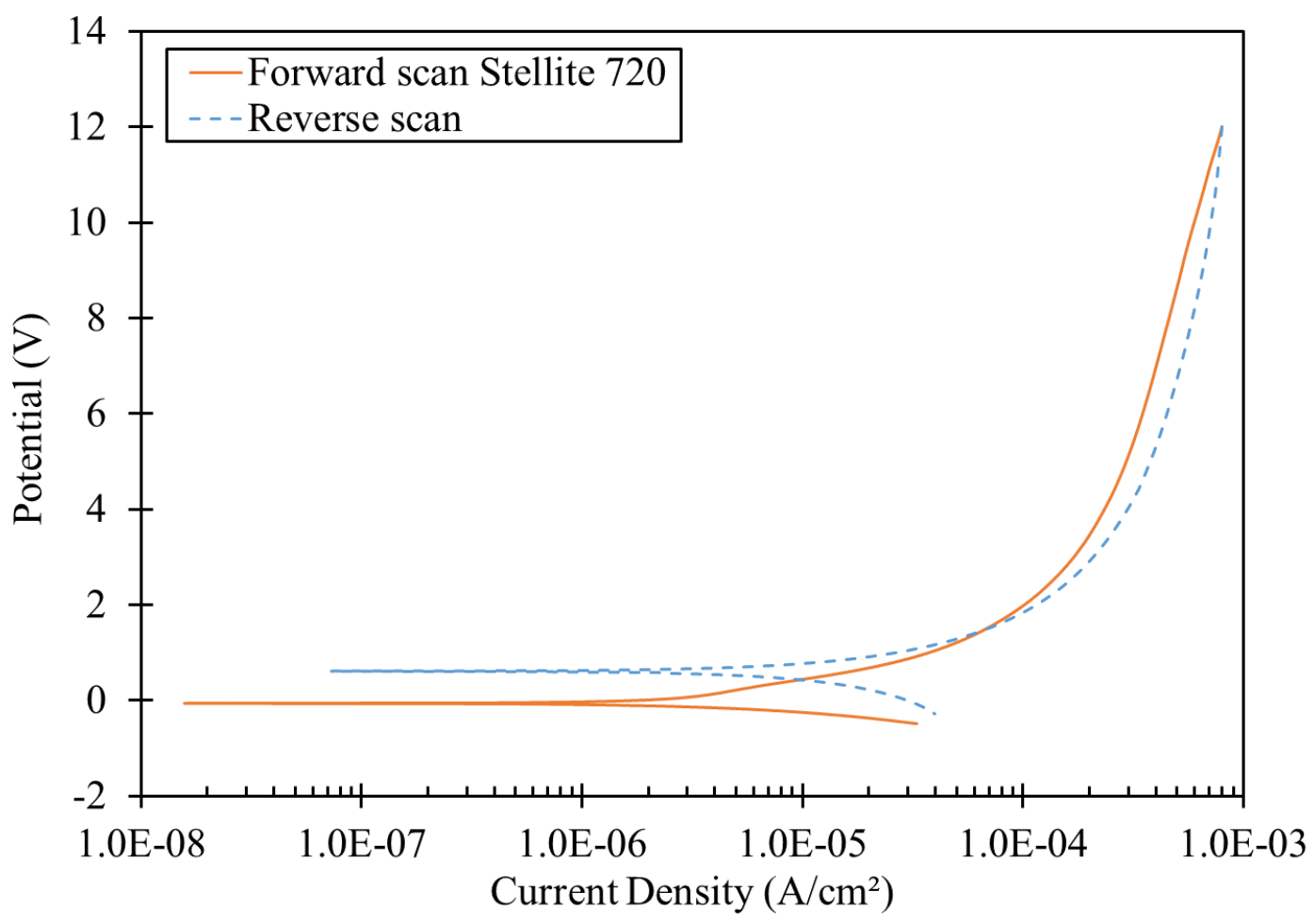

Figure 4-58: Cyclic polarization of Stellite 720 under peak voltage of $12 \mathrm{~V}$ in morpholine 


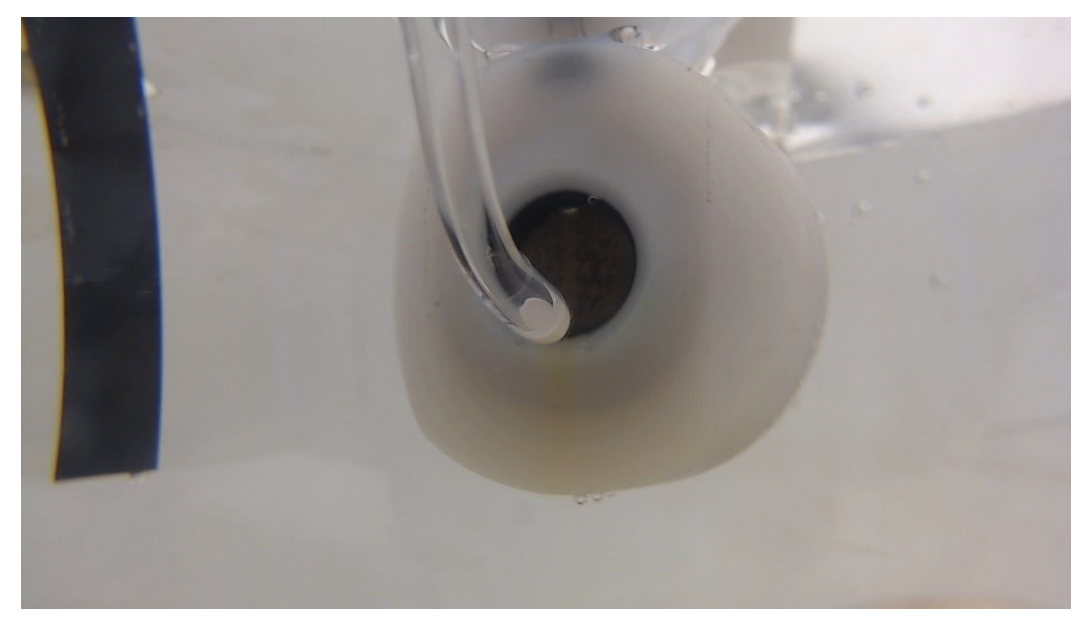

Figure 4-59: Stellite 706 surface after exposure of up to $12 \mathrm{~V}$ in morpholine

\subsubsection{Corrosion performance in $3.5 \mathrm{wt} . \% \mathrm{NaCl}$}

\subsubsection{Potentiodynamic polarization}

To further investigate the limitations of the Stellite alloys and influence of chemical composition on the corrosion behaviour, $3.5 \mathrm{wt} . \% \mathrm{NaCl}$ was also used as a substitute for morpholine. With a more conductive corrosive solution, a clear difference can be seen amongst the alloy in terms of their localized corrosion behaviour. The scan rate remained the same at $25 \mathrm{mV} / \mathrm{s}$ and the peak potentials were between $2-8 \mathrm{~V}$. Due to the limitations of the machine once a peak current value of $2.5 \mathrm{~A}$ is reached, the reverse scan starts before reaching the set peak potential value, hence the tests can only be done up to $8 \mathrm{~V}$. As shown in Figure 4-60, the alloys became more active with the current density going above 2 A. The alloy surface started rapid dissolution, which was visually observed above 1.2 V potential as shown in Figure 4-61 using Stellite 706 as an example. Small fluctuation can be observed above $4 \mathrm{~V}$ potential, which is an indication of pitting happening on the alloy surface due to high current activity. 


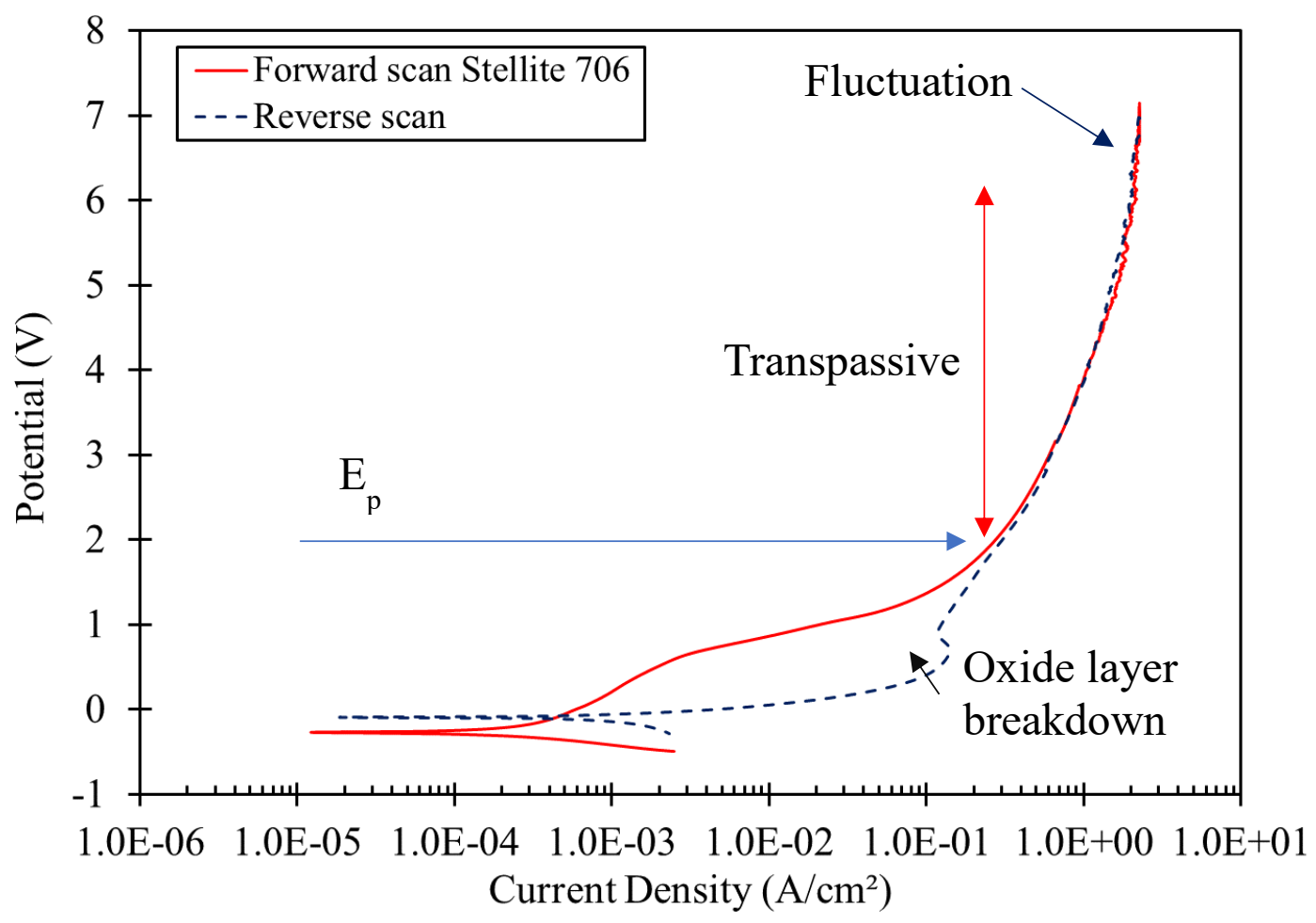

(a)

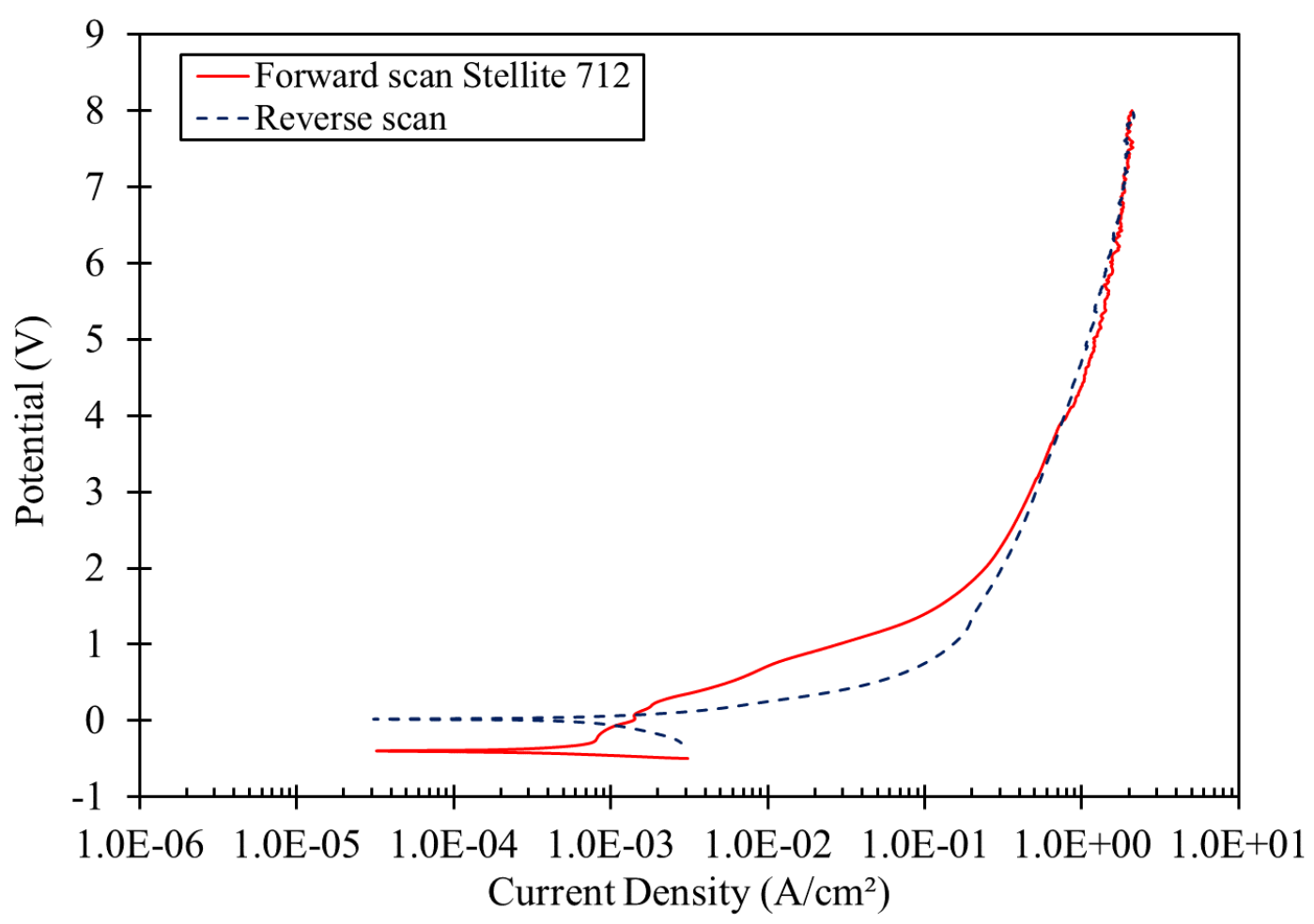

(b) 


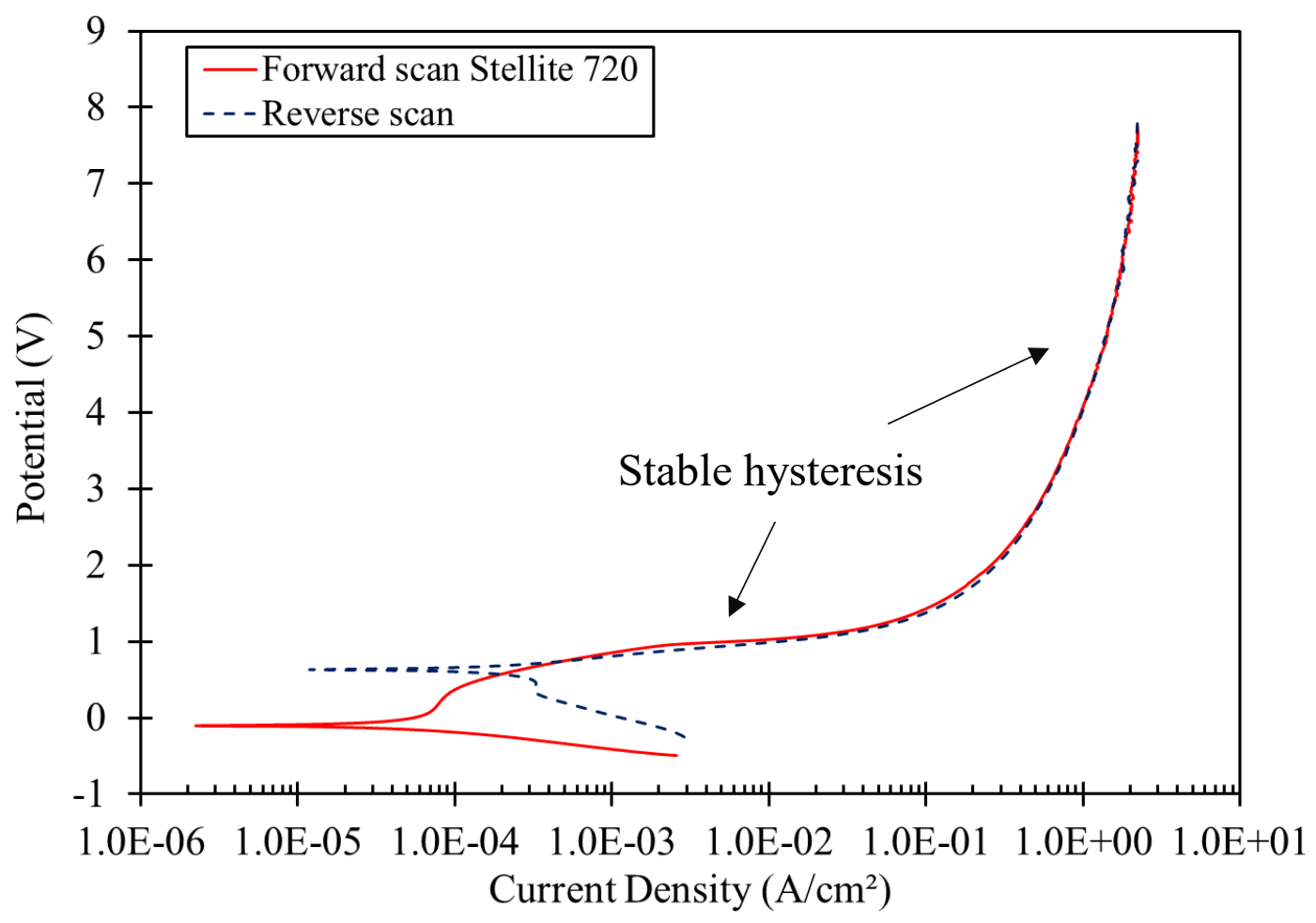

(c)

Figure 4-60: Cyclic polarization curves of (a) Stellite 706, (b) Stellite 712 and (c) Stellite 720 tested in 3.5 wt.\% $\mathrm{NaCl}$ under the peak voltage of $8 \mathrm{~V}$

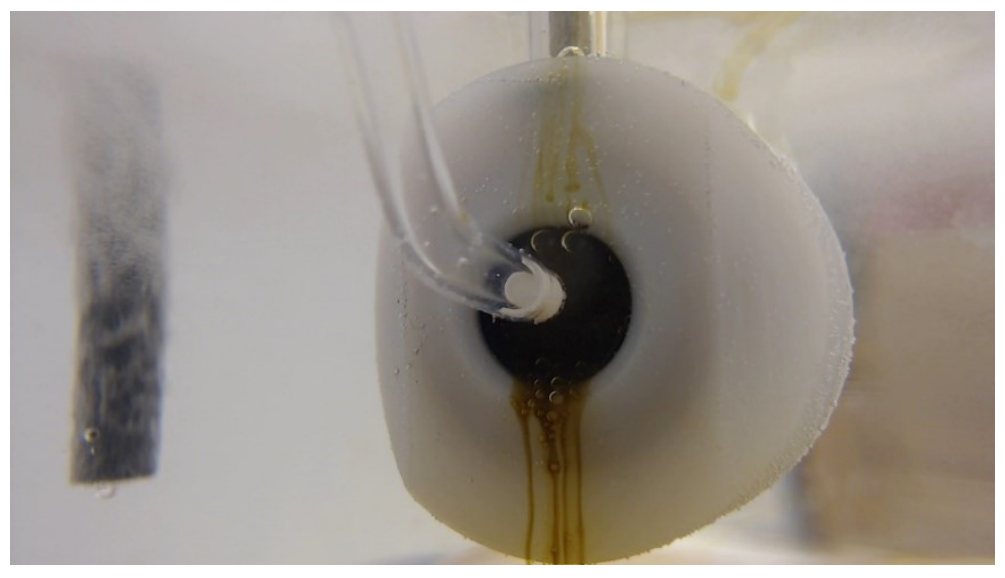

Figure 4-61: Stellite 706 surface corrosion in $3.5 \mathrm{wt} \% \mathrm{NaCl}$ solution 
In comparison among the three alloys, the most significant noticeable difference is the localized corrosion behaviour. Stellite 706 and Stellite 712 displayed oxide film breakdown below the $E_{p}$ in its reverse scan. The hysteresis is stable starting from the onset of the peak potential down to the $E_{p}$, however, it displays negative hysteresis once below the $E_{p}$. This breakdown is not observed in Stellite 720 as it is able to maintain its oxide film integrity with the hysteresis showing a stable behaviour entirely. This further verifies the influence of Mo content in Stellite, where Stellite 720 having the higher Mo at 18 wt.\% displayed the best performance. All tests done for every peak voltage displayed the same behaviour.

\subsubsection{EIS test}

As seen from Figure 4-60, Stellite 706 and Stellite 712 experienced negative hysteresis below 2 V, whereas Stellite 720 displayed continuous stability across the whole test. Furthering this study, EIS test was conducted on these alloys with a DC voltage within $0.2-1.2 \mathrm{~V}$ to observe the behaviour of the oxide layer within the negative hysteresis displayed in Figure 4-60(a) and (b). DC potential was applied up until $1.2 \mathrm{~V}$ in $0.2 \mathrm{~V}$ increments and then the voltage was reversed back to $0 \mathrm{~V}$ DC. The curves were obtained for each voltage in Figure 4-62 where the fluctuation was an indication of oxide film breakdown where pseudo-capacitive behaviour is no longer possible. All reverse scan impedance lines shifted downwards while maintaining a capacitive level with the same slope indicating that the oxide thickness was smaller compared to the beginning. A clear difference is seen among the curves in Figure 4-60 for the three alloys where Stellite 720 shows better stability in oxide retention because fluctuation is not observed until $1 \mathrm{~V}$ DC potential, whereas Stellite 706 and Stellite 712 start displaying fluctuation around 0.6 V DC potential. 


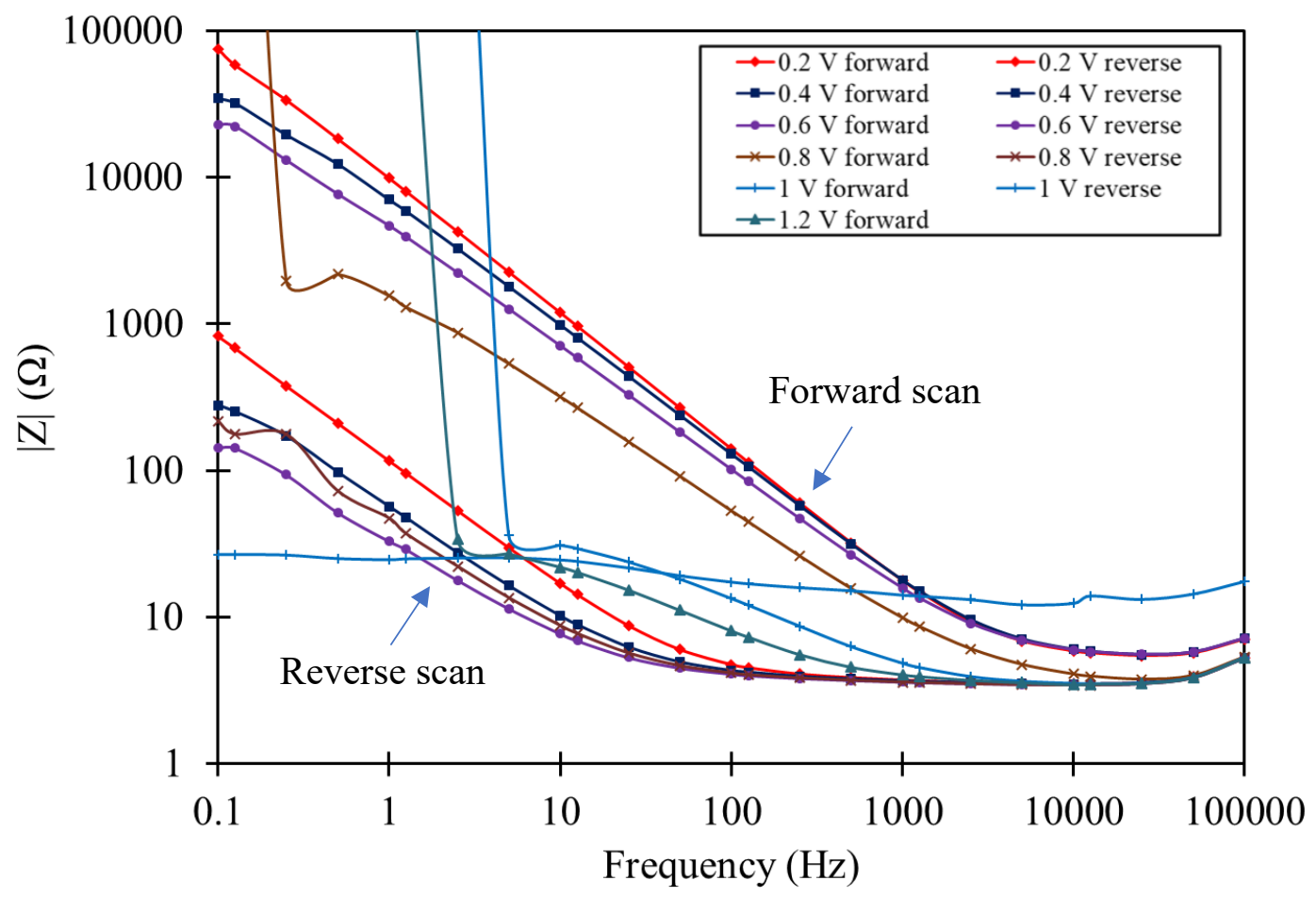

(a)

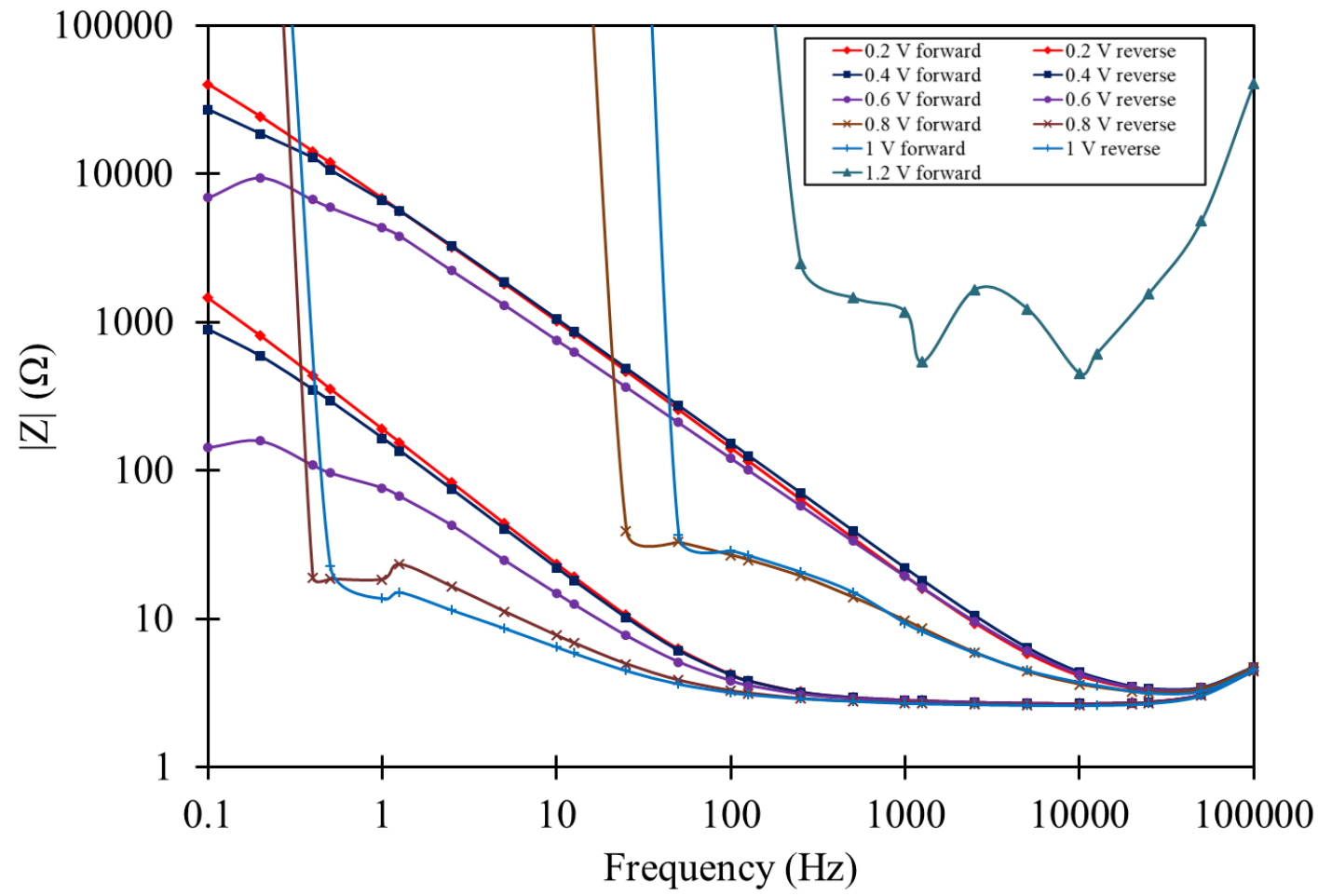

(b) 


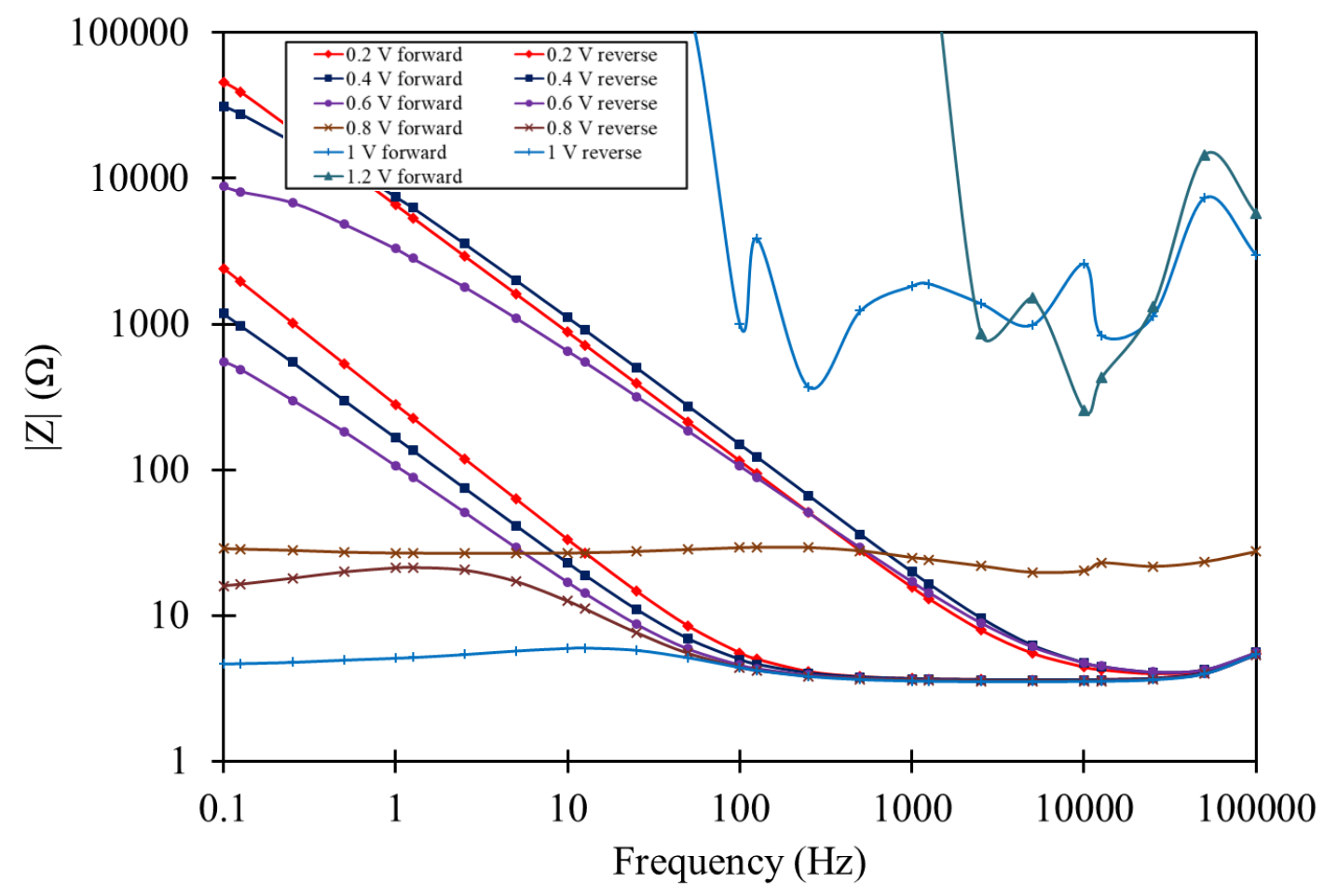

(c)

Figure 4-62: Impedance variations within the negative hysteresis range for (a) Stellite 706, (b) Stellite 712 and (c) Stellite 720

The early fluctuation from the forward scan in Stellite 706 and Stellite 712 indicate early oxide film breakdown and fluctuations in the reverse scan indicate slower recovery. Stellite 720 at $0.8 \mathrm{~V}$ showed a straight horizontal line indicating resistive behaviour, where the oxide layer was still stable enough to resist corrosion because it was not yet broken. This EIS test served as a confirmation that Stellite 720 can better retain oxide stability in an aggressive $\mathrm{NaCl}$ environment.

The concepts mentioned in the literature review about Mo influence in stainless steels can be applied to Stellite alloys, where $\mathrm{Cr}$ and Mo are major alloying elements for corrosion protection. In many industries such as oil and gas, pitting resistance equivalent number (PREN) is a parameter used to theoretically compare pitting corrosion resistance of stainless-steel alloys [33, 34]. The 
most common cause of pitting is attacks from acids and salt water in which $\mathrm{Cr}$ reacts readily with chloride ions to produce chromium chloride $\left(\mathrm{CrCl}_{3}\right)$ [34]. PREN number involves adding the compositions of three common alloying elements that are used as counter measures for pitting corrosion such as $\mathrm{Cr}$, Mo and nitrogen $(\mathrm{N})$ in stainless steels displayed in Eq (17) [33, 34]:

$$
\begin{aligned}
& \text { PREN }=\% C r+3.3(\% M o)+16(\% N) \\
& \text { PREN }=\% C r+3.3(\% M o+0.5 \% W)
\end{aligned}
$$

The percentage refers to the weight percent of each element. Since $\mathrm{N}$ is not an element used in Stellite, it can be removed from Eq (17) leaving the essential elements used in Stellite for combating corrosion in Eq (18). This equation can be used to rank pitting susceptibility of the Stellite alloys conducted for this research. Using Eq (18) the PREN for each Stellite alloy was calculated in Table 4-11. With Stellite 720 having the highest PREN, it can be a reasonable explanation for its better oxide stability in $3.5 \mathrm{wt} . \% \mathrm{NaCl}$ solution. Given what has already been discussed in Section 2.3, the $\mathrm{Mo}^{4+}$ and $\mathrm{Mo}^{6+}$ valence ions can play a role in forming a complex with chloride ions to reduce pitting and help with oxide film stability and recovery at higher potentials.

Table 4-11: PREN for each Stellite alloy

\begin{tabular}{|c|c|}
\hline Alloy & PREN \\
\hline Stellite 706 & 45.5 \\
\hline Stellite 712 & 57.05 \\
\hline Stellite 720 & 92.4 \\
\hline Stellite 6 & 41.375 \\
\hline
\end{tabular}




\subsection{Comparison with Stellite 6}

Stellite 6 has been the alloys of choice for hardfacing valve trim components due to its excellent combined mechanical, corrosion, wear, and high-temperature properties. To better assess the corrosion performance of 700 series Stellite alloys in morpholine, the experimental results of these alloys are compared with that of Stellite 6 under the same test conditions.

\subsubsection{Chemical composition and microstructure}

Stellite 6 and Stellite 706 are initially compared due to their compositional similarity. Stellite 6 has a chemical composition of $(29 \% \mathrm{Cr}, 4.5 \% \mathrm{~W}, 1.5 \% \mathrm{Mo}, 1.2 \% \mathrm{C}$, in weight $)$ like that of Stellite $706(29 \% \mathrm{Cr}, 5 \% \mathrm{Mo}, 1.2 \% \mathrm{C})$. The difference is the additional $\mathrm{W}$ content and less Mo content. The SEM image of Stellite 6 is compared with that of Stellite 706 in Figure 4-63. These two alloys have a similar microstructure with $\mathrm{Cr}_{7} \mathrm{C}_{3}$ dendritic carbides embedded in the matrix, however, the white region in Stellite 6 is $(\mathrm{W}, \mathrm{Co})_{6} \mathrm{C}$ as opposed to $(\mathrm{Co}, \mathrm{Mo})_{3} \mathrm{C}$ in Stellite 706.

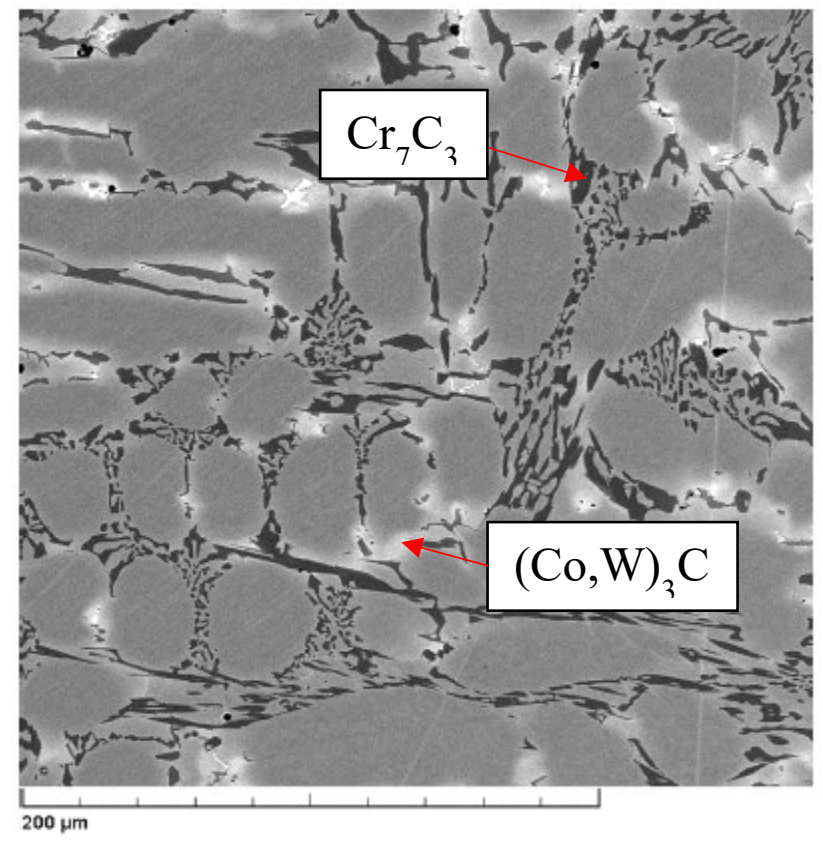

(a)

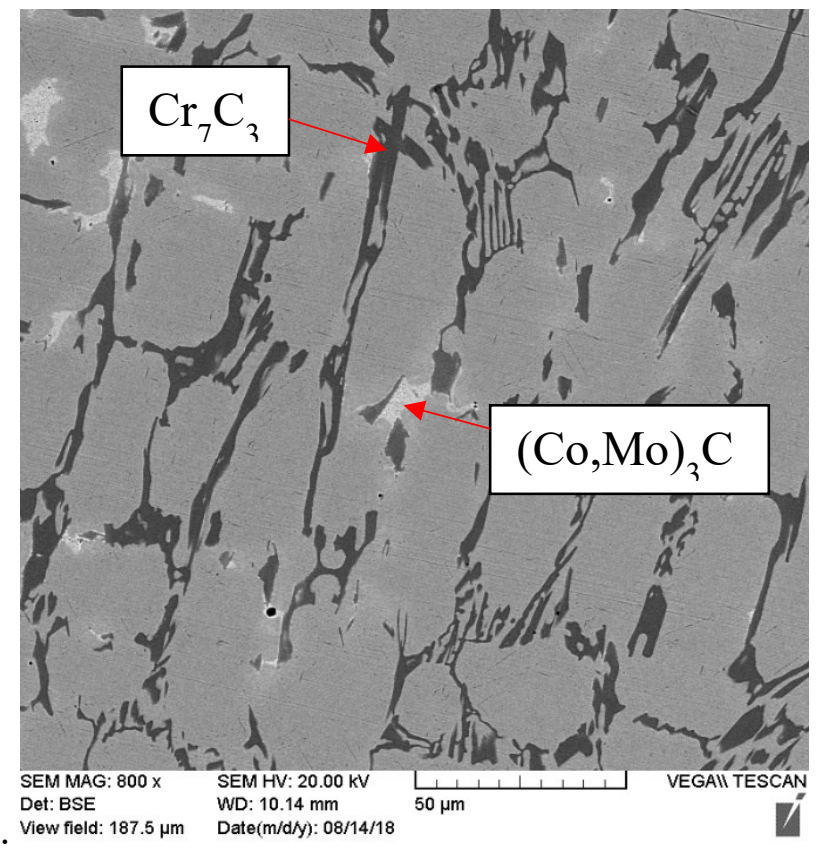

(b)

Figure 4-63: SEM microstructure of (a) Stellite 6 [5] and (b) Stellite 706 


\subsubsection{EIS and cyclic polarization}

The EIS trend comparison between Stellite 6 and Stellite 706 is shown in Figure 4-64 for all three OCP durations. Qualitatively the differences are not distinguishable, however quantitatively from Table 4-12, the $R_{p}$ values for Stellite 706 are larger. Stellite 6 is seen to reach its peak performance in morpholine after 24 hours OCP where its performance did not enhance after 48 hours. With Stellite 706 having higher Mo content, the resistance of the oxide in comparison is much stronger. Figure 4-65 show bar graphs representing $R_{p}$ for both alloys where Stellite 706 after 24 and 48 hours is clearly more resistant to charge transfer.

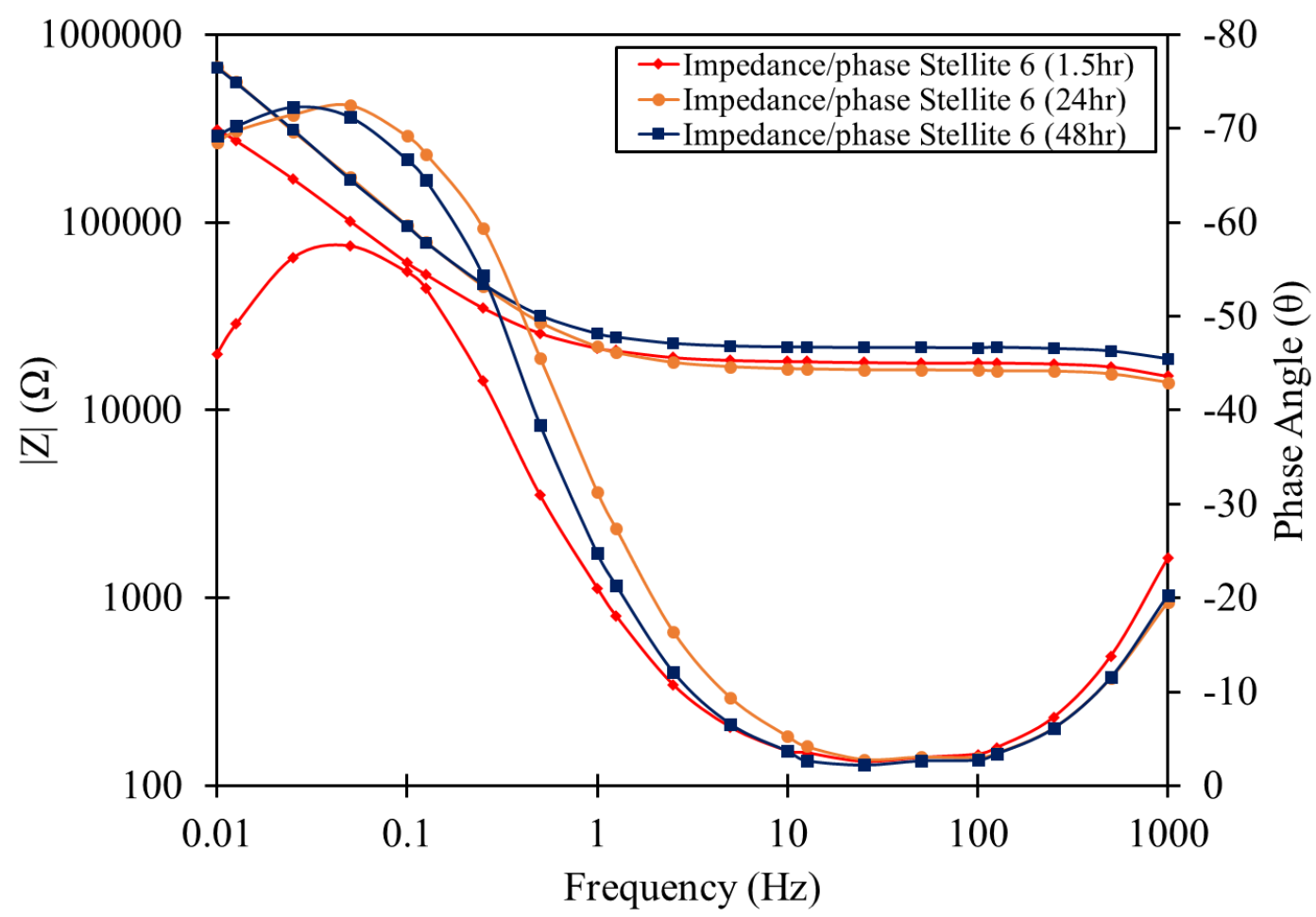

(a) 


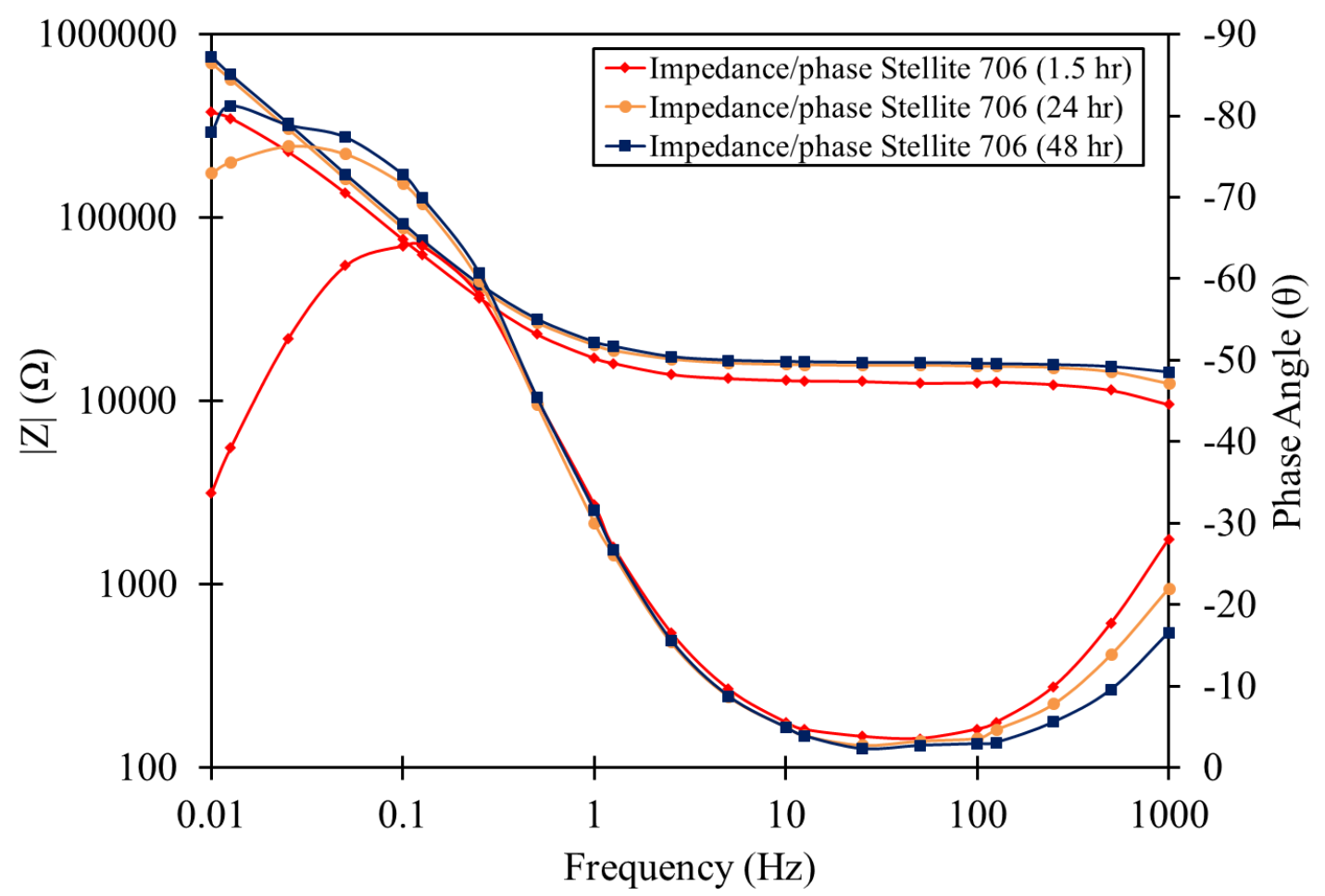

(b)

Figure 4-64: EIS comparison for all OCP durations between (a) Stellite 6 and (b) Stellite 706

Table 4-12: EIS result comparison between Stellite 6 and Stellite 706

\begin{tabular}{|c|c|c|c|c|c|}
\hline Time (hr) & Alloy & $\mathbf{1 0}^{-\mathbf{6}} \mathbf{x} \mathbf{C P E}\left(\mathbf{\Omega}^{-\mathbf{1}} \mathbf{s}^{\mathbf{n}}\right)$ & $\mathbf{n}$ & $\mathbf{R}_{\mathbf{p}} \mathbf{( k \Omega )}$ & $\mathbf{R}_{\mathbf{s}}(\mathbf{k} \mathbf{\Omega})$ \\
\hline \multirow{2}{*}{1.5} & Stellite 706 & $20.8 \pm 0.1$ & $0.91 \pm 0.004$ & $689 \pm 23$ & $13.6 \pm 0.1$ \\
\cline { 2 - 6 } & Stellite 6 & $19.1 \pm 0.1$ & $0.89 \pm 0.002$ & $607 \pm 6$ & $18.1 \pm 0.04$ \\
\hline \multirow{2}{*}{24} & Stellite 706 & $16.2 \pm 0.1$ & $0.91 \pm 0.001$ & $6579 \pm 156$ & $13.6 \pm 0.06$ \\
\cline { 2 - 6 } & Stellite 6 & $17.5 \pm 0.04$ & $0.90 \pm 0.001$ & $3653 \pm 140$ & $17.6 \pm 0.04$ \\
\hline \multirow{2}{*}{48} & Stellite 706 & $15.7 \pm 0.1$ & $0.92 \pm 0.001$ & $9226 \pm 403$ & $14.7 \pm 0.05$ \\
\cline { 2 - 6 } & Stellite 6 & $18.1 \pm 0.04$ & $0.88 \pm 0.001$ & $3470 \pm 92$ & $17.9 \pm 0.04$ \\
\hline
\end{tabular}




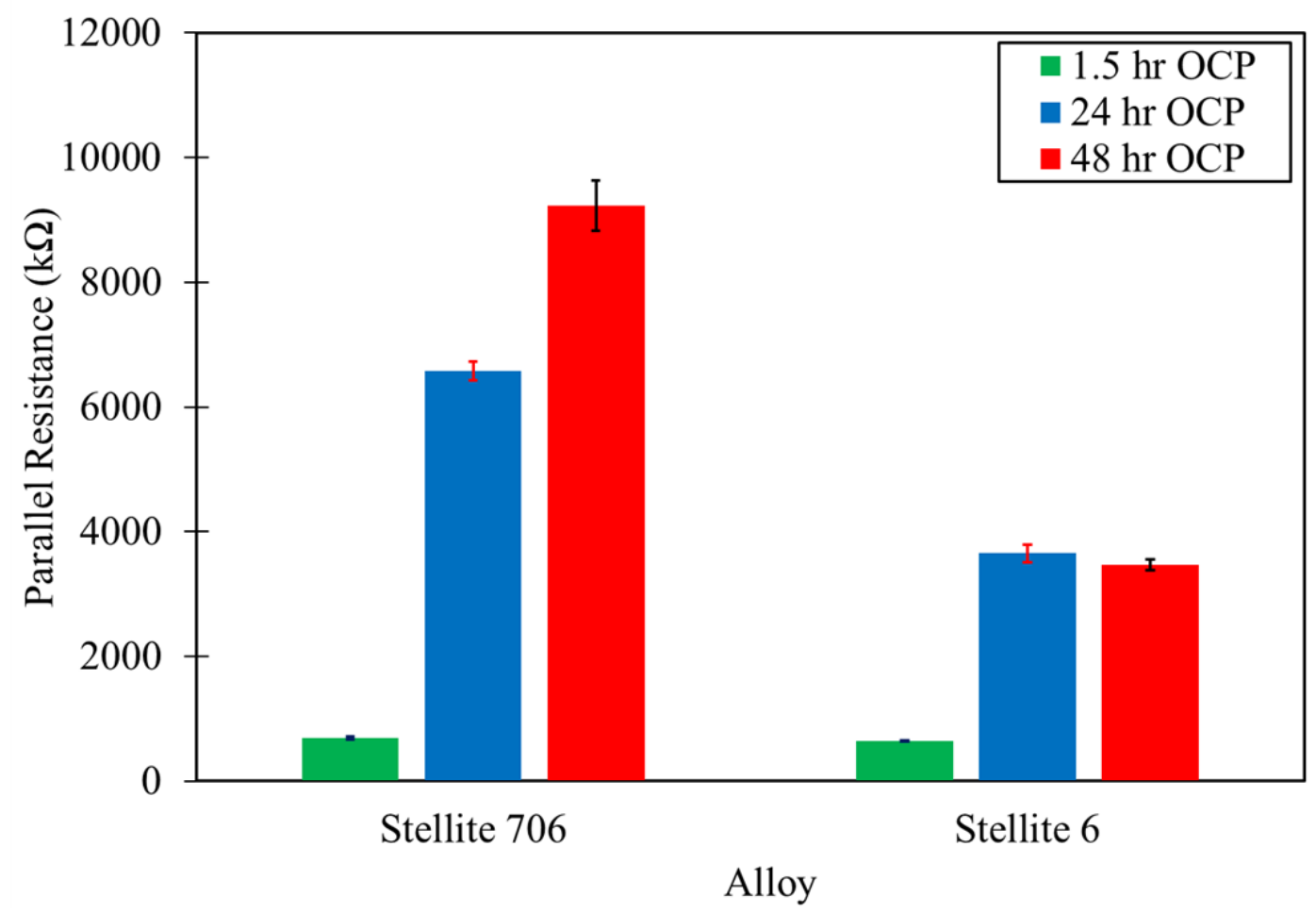

Figure 4-65: Comparison of $\boldsymbol{R}_{p}$ between Stellite 6 and Stellite 706

Cyclic polarization forward curve comparison is given in Figure 4-66, where once again qualitatively they both look similar in all OCP durations. Spontaneous passivity is not readily seen in Stellite 6, but the point at which transpassive behaviour starts is like Stellite 706. The maximum current density reached is also similar where it is less than $10^{-4} \mathrm{~A}$. Quantitative results in Table 4-13 show analogous results compared to EIS. General corrosion performance for Stellite 6 remains the same as $R_{p}$ does not increase after 48 hours OCP. Figure 4-67 shows the bar graph comparison of $R_{p}$, where Stellite 6 does not show an increase in performance analogous to what is seen in Figure 4-65. Hysteresis comparison in Figure 4-68 shows that Stellite 6 has stable localized corrosion behaviour for all OCP durations. 


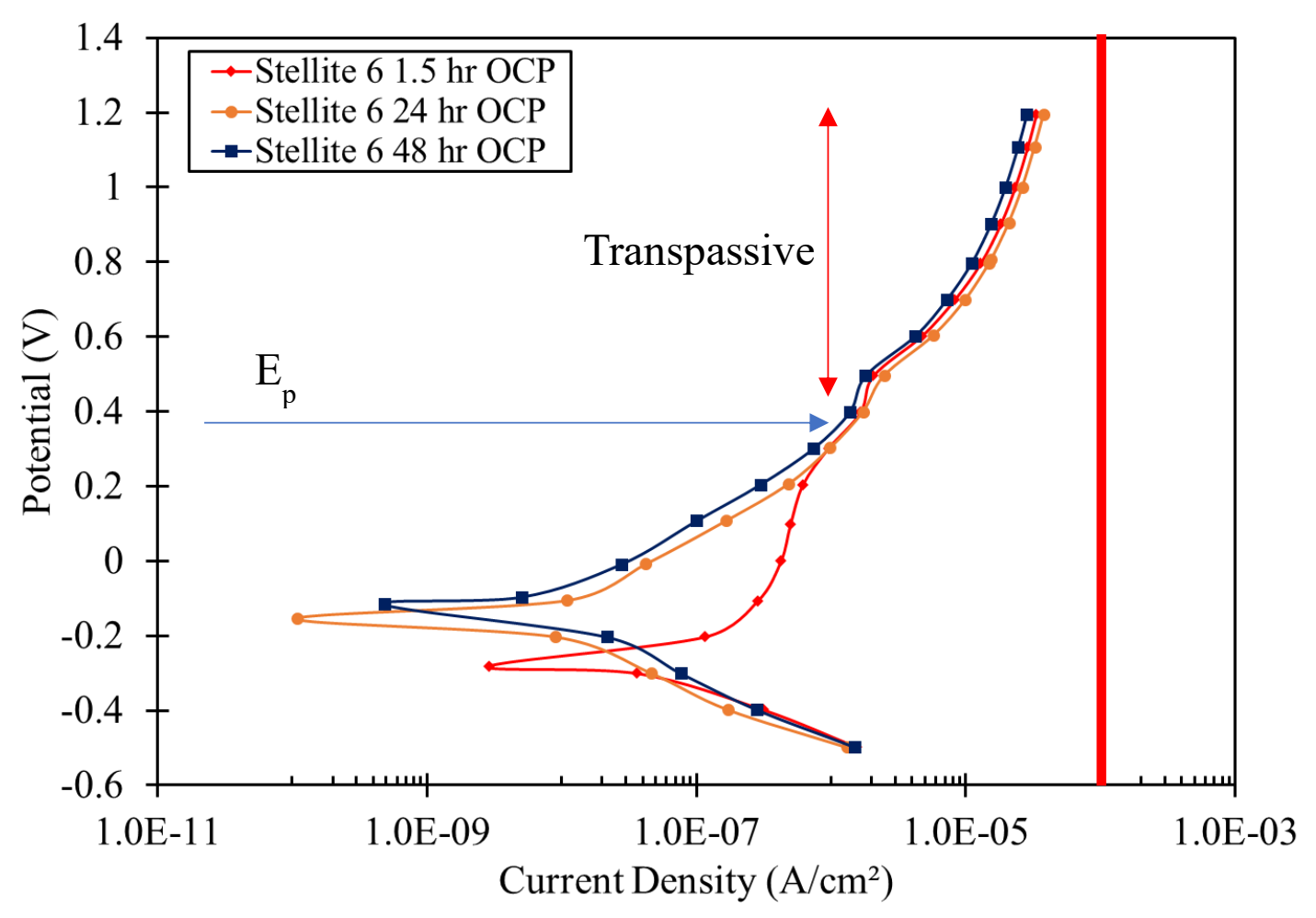

(a)

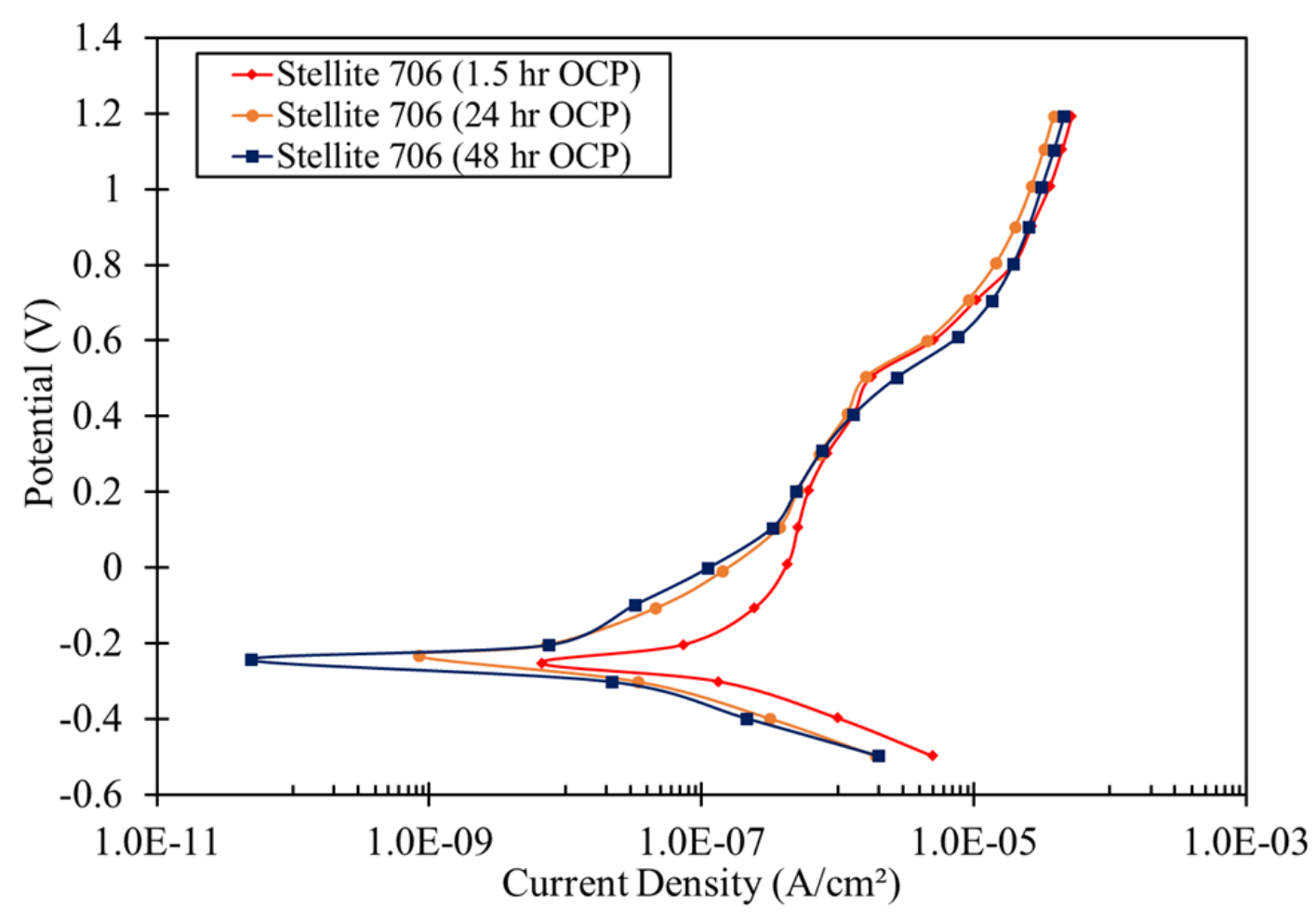

(b)

Figure 4-66: Polarization curves comparison between (a) Stellite 6 and (b) Stellite 706 
Table 4-13: Polarization result comparison between Stellite 6 and Stellite 706

\begin{tabular}{|c|c|c|c|c|c|}
\hline Time (hr) & Alloy & OCP $(\mathbf{m V})$ & $\mathbf{E}_{\text {corr }}(\mathbf{m V})$ & $I_{\text {corr }}\left(\mathbf{n A} / \mathbf{c m}^{\mathbf{2}}\right)$ & $\mathbf{R}_{\mathbf{p}}\left(\mathbf{k} \mathbf{\Omega} \cdot \mathbf{c m}^{\mathbf{2}}\right)$ \\
\hline \multirow{2}{*}{1.5} & Stellite 706 & $-298 \pm 14$ & $-289 \pm 36$ & $47.1 \pm 2$ & $553 \pm 30$ \\
\cline { 2 - 6 } & Stellite 6 & $-272 \pm 13$ & $-291 \pm 16$ & $40.4 \pm 3$ & $646 \pm 54$ \\
\hline \multirow{2}{*}{24} & Stellite 706 & $-223 \pm 22$ & $-252 \pm 27$ & $9.3 \pm 0.1$ & $2807 \pm 21$ \\
\cline { 2 - 6 } & Stellite 6 & $-157 \pm 3$ & $-172 \pm 2$ & $6.2 \pm 0.1$ & $4212 \pm 37$ \\
\hline \multirow{2}{*}{48} & Stellite 706 & $-179 \pm 28$ & $-224 \pm 14$ & $6.1 \pm 0.2$ & $4245 \pm 132$ \\
\cline { 2 - 6 } & Stellite 6 & $-73 \pm 1$ & $-115 \pm 3$ & $6.2 \pm 0.2$ & $4226 \pm 128$ \\
\hline
\end{tabular}

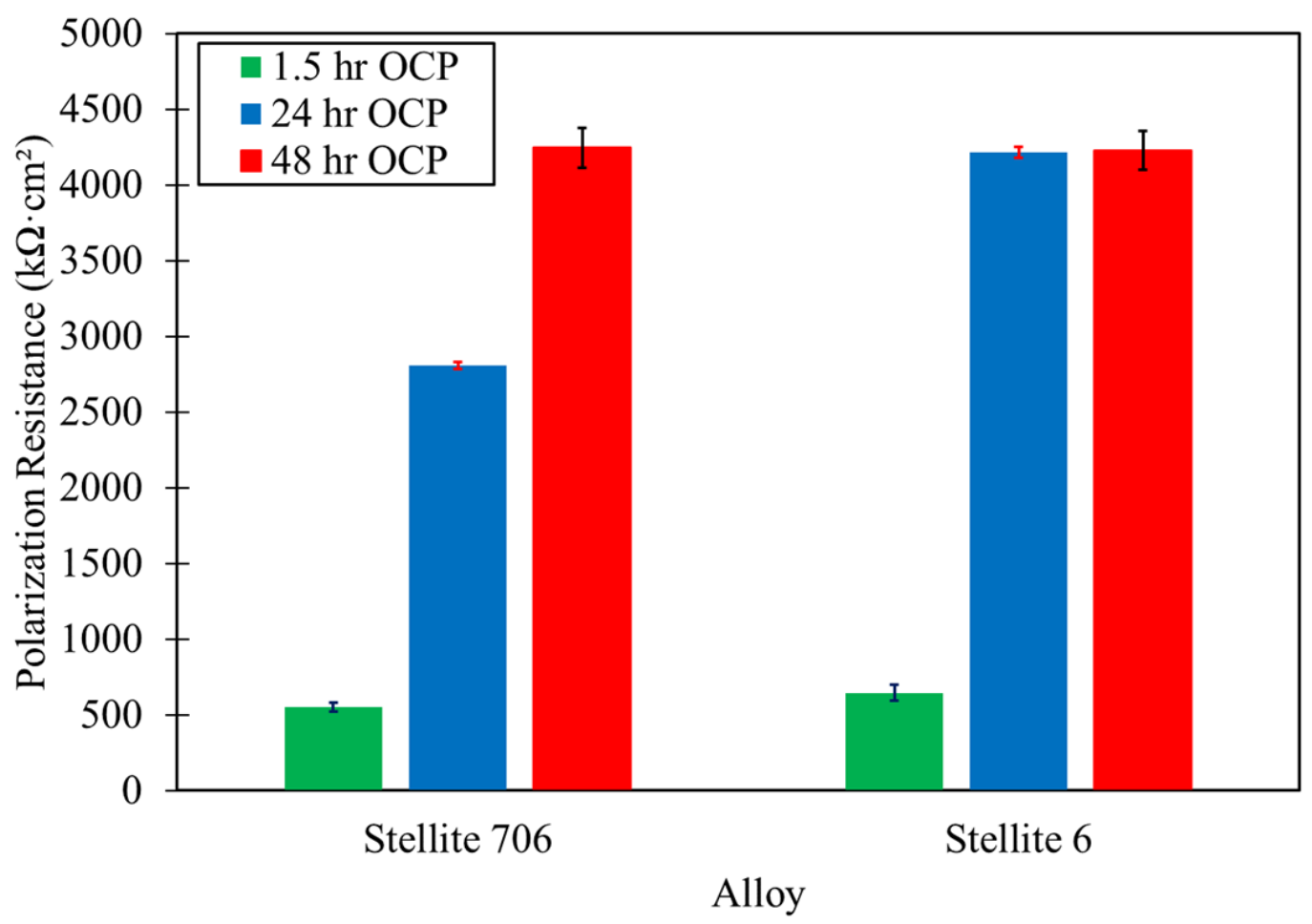

Figure 4-67: Comparison of $R_{p}$ between Stellite 6 and Stellite 706 


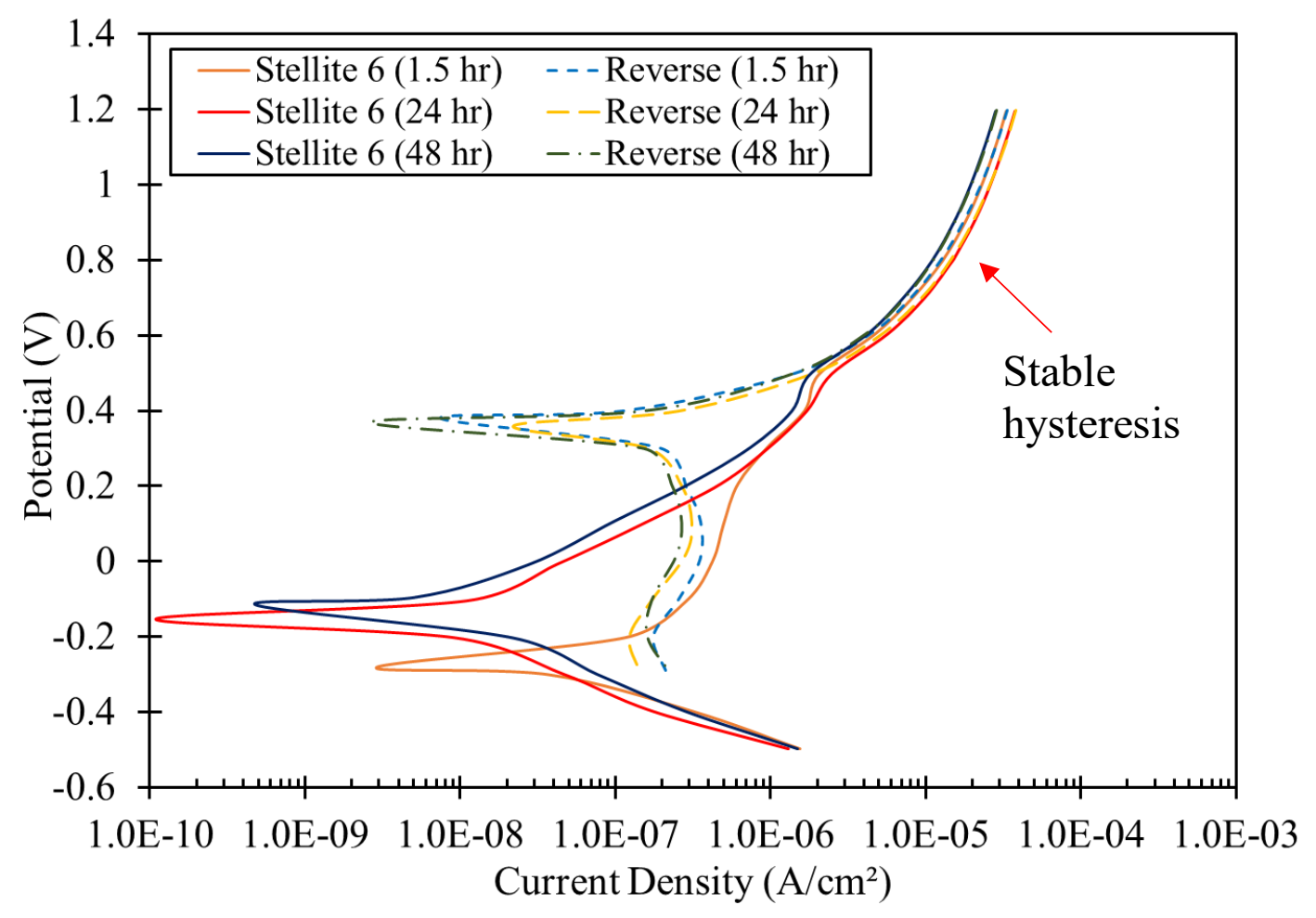

(a)

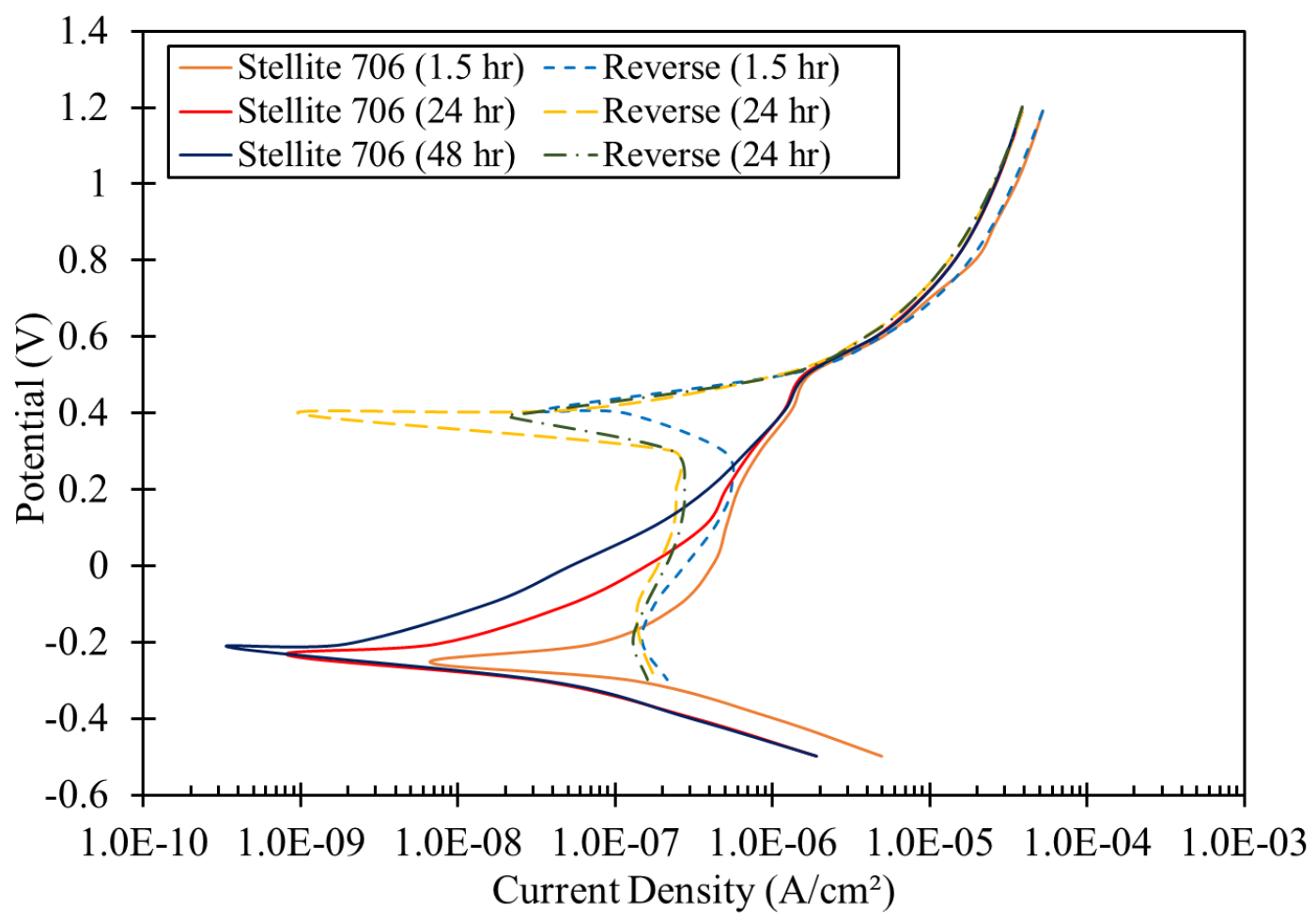

(b)

Figure 4-68: Hysteresis comparison between (a) Stellite 6 and (b) Stellite 706 


\subsubsection{Failure test}

Failure test is done on Stellite 6 with both morpholine at $\mathrm{pH} 9.5$ and $3.5 \mathrm{wt} . \% \mathrm{NaCl}$ at a high scan rate of $25 \mathrm{mV} / \mathrm{s}$. The test is started from $-0.5 \mathrm{~V}$, taken up to $2-12 \mathrm{~V}$ peak potential and ended at $-0.3 \mathrm{~V}$. All tests with peak potentials between $2-12 \mathrm{~V}$ show similar trend from start to finish. From Figure 4-69, Stellite 6 has a slightly larger gap in its hysteresis behaviour, however, the gap is not significant as it is fairly like Stellite 706. The maximum current density reached is $10^{-3} \mathrm{~A}$ and by comparison with Figure 4-57 and Figure 4-58, morpholine does not contribute to accelerating corrosion of all the Stellite alloys tested. Figure 4-70 shows the surface condition of Stellite 6 after applying $12 \mathrm{~V}$ potential. With the small color change, it is an indication that oxidation did occur, but at a very small scale.

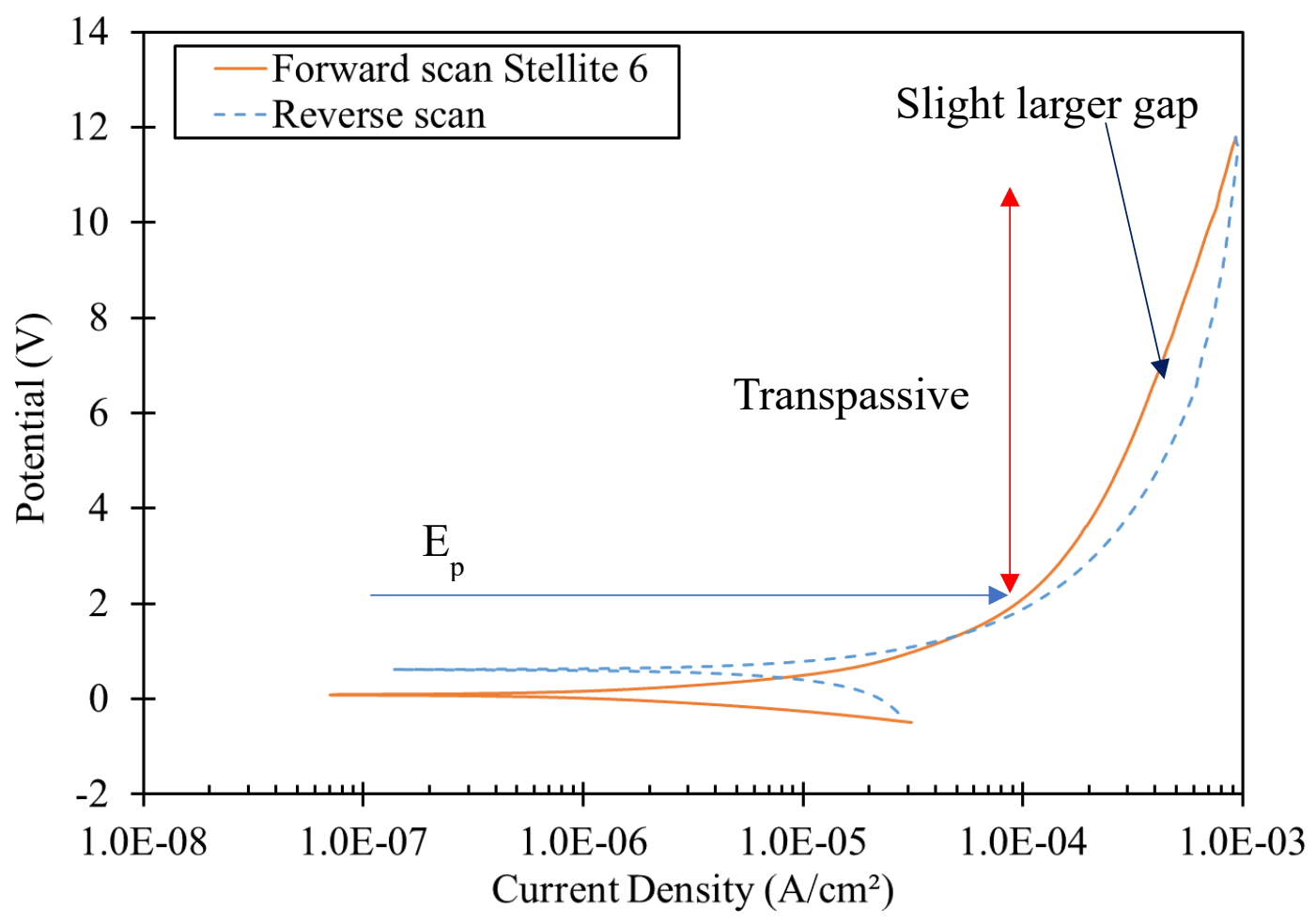

(a) 


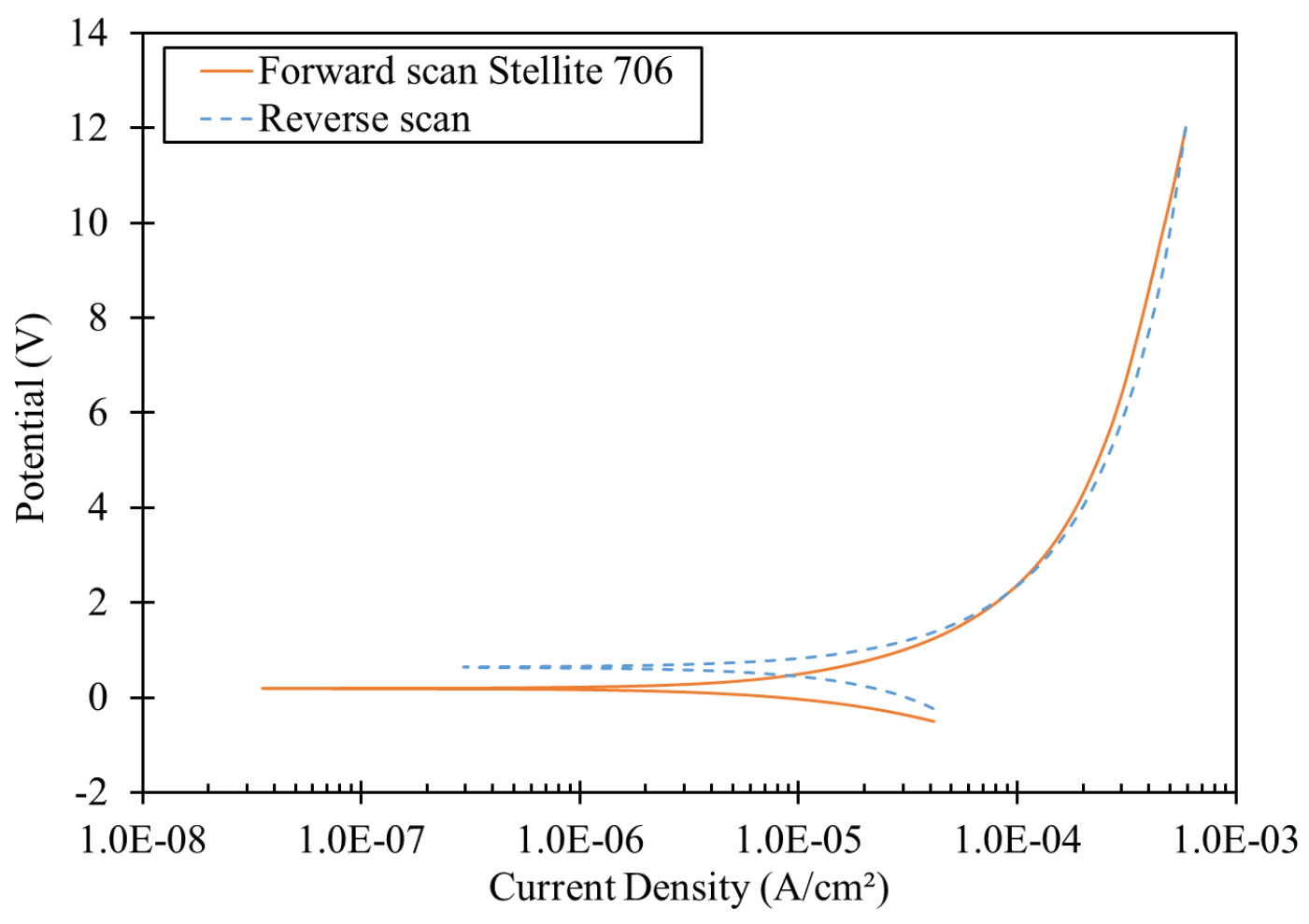

(b)

Figure 4-69: Cyclic polarization comparison between (a) Stellite 6 and (b) Stellite 706 in morpholine

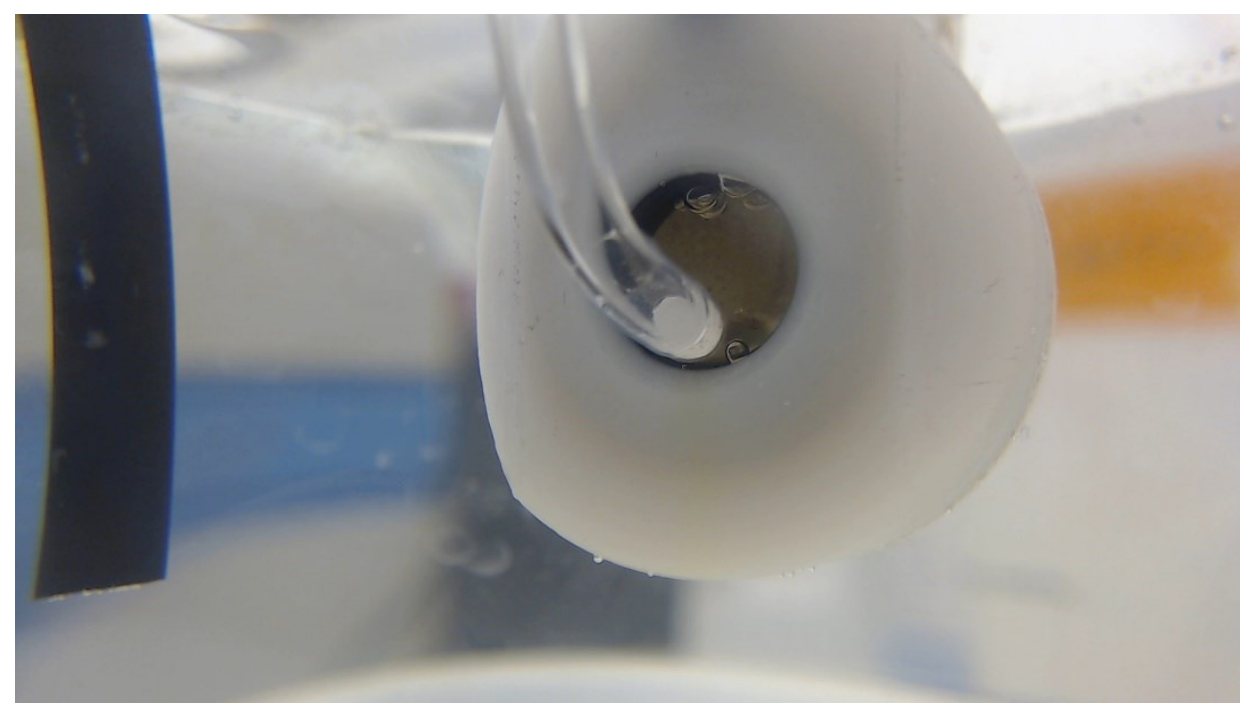

Figure 4-70: Stellite 6 after $12 \mathrm{~V}$ failure test in morpholine 
Comparison in $\mathrm{NaCl}$ is done at the same conditions where the test could not be done beyond $8 \mathrm{~V}$ due to instrument limitations. In Figure 4-71, Stellite 6 displays similar localized corrosion behaviour at the higher potentials compared to Stellite 706 . From a stable hysteresis, the oxide integrity is lost once the reverse scan goes below the $E_{p}$. With the inclusion of W in Stellite 6, it seemed to have made little difference in terms of localized corrosion performance in an aggressive $\mathrm{NaCl}$ environment.

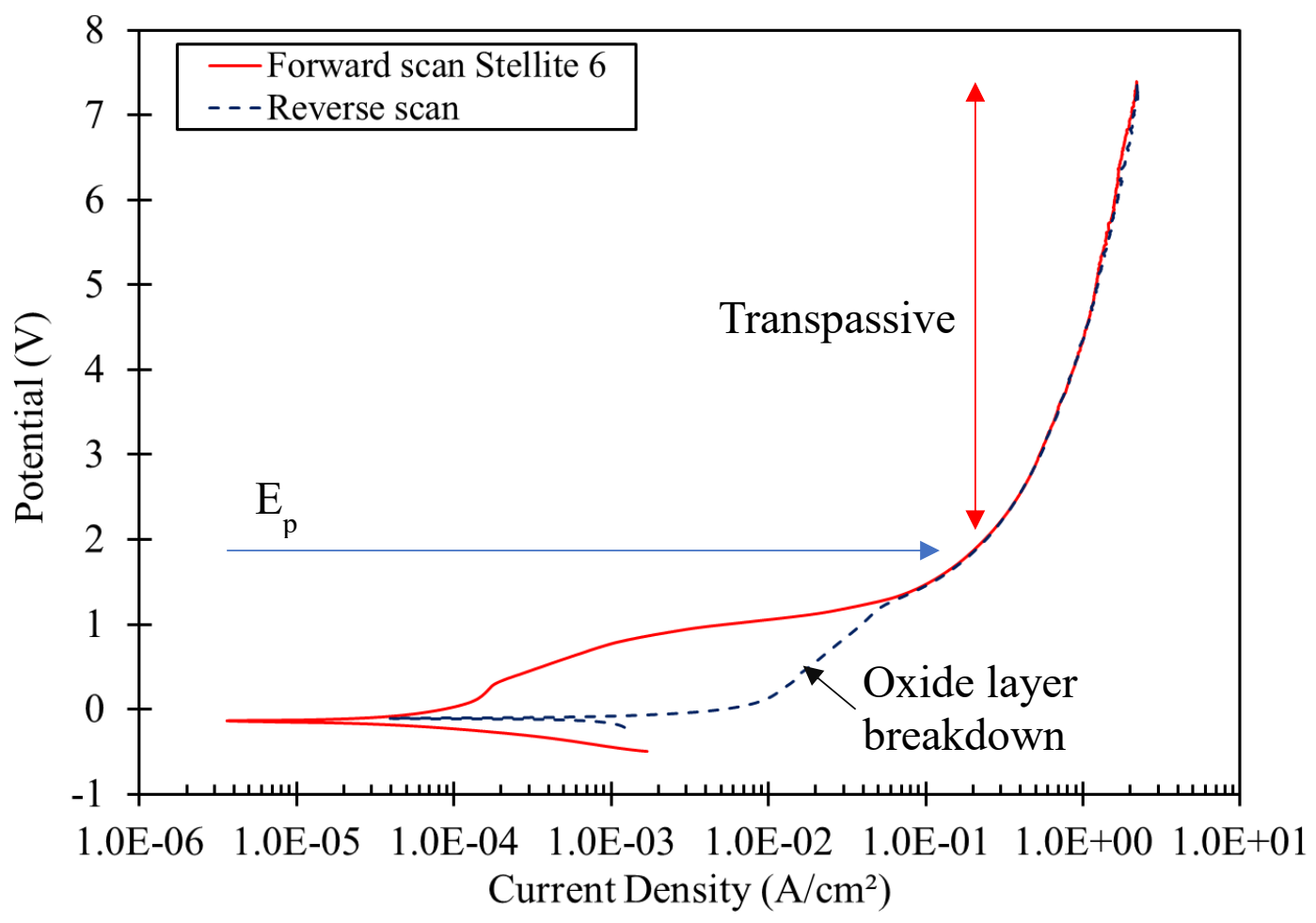

(a) 


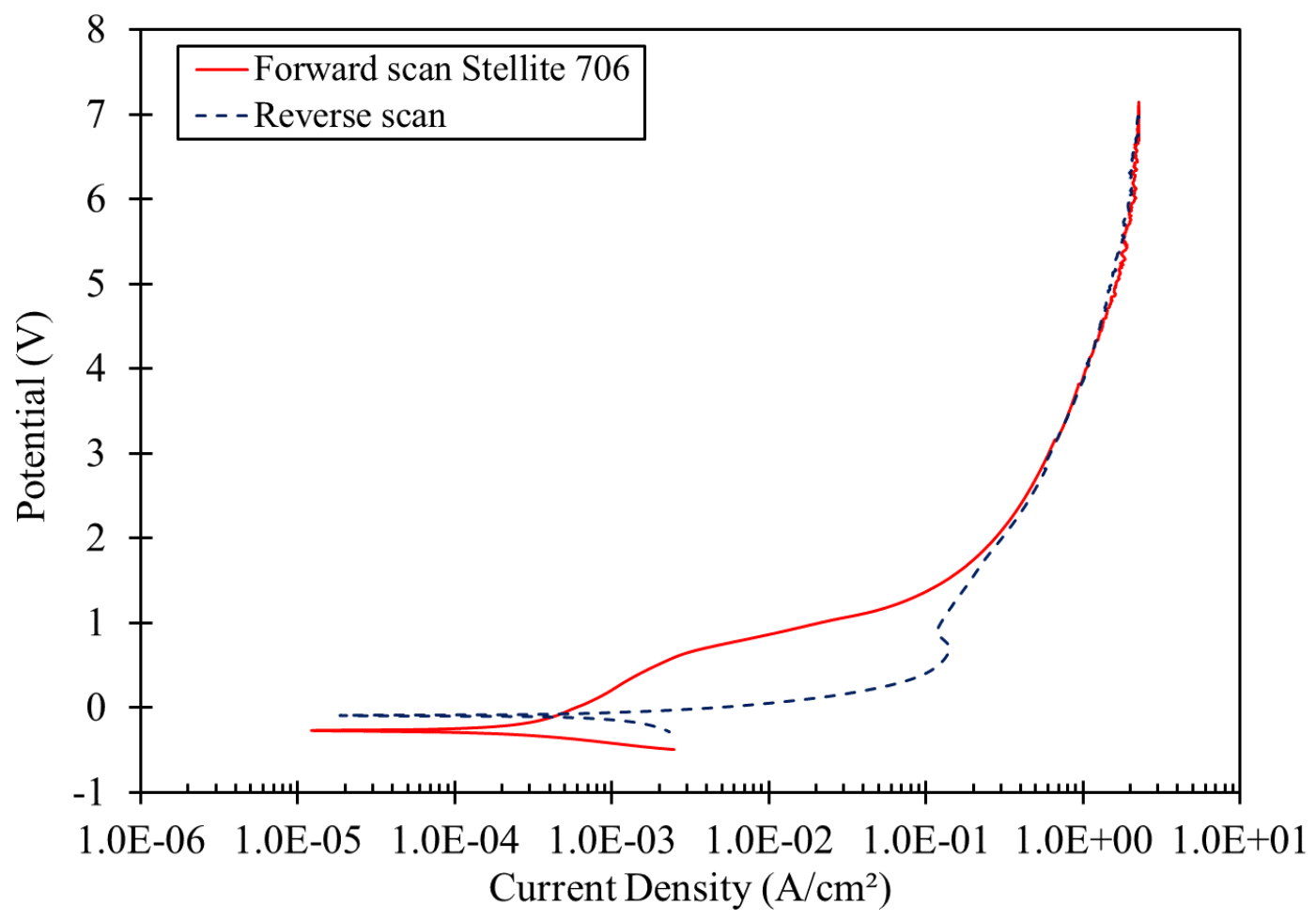

Figure 4-71: Cyclic polarization comparison between (a) Stellite 6 and (b) Stellite 706 in 3.5 wt.\% NaCl

\subsubsection{Overall comparison}

An overall comparison can be made amongst all the Stellite alloys to properly distinguish their performance. Figure 4-72 shows the $R_{p}$ behaviour of all Stellite alloys from EIS and polarization at 48 hours OCP. From the bar graph comparison, Stellite 720 overall has the best performance in both its oxide layers ability to impede charge transfer and general corrosion resistance in morpholine. In addition, Stellite 720 with the greatest amount of Mo content at $18 \mathrm{wt} \% \%$ has the optimum composition for best performance in localized corrosion resistance in an aggressive $\mathrm{NaCl}$ environment. 


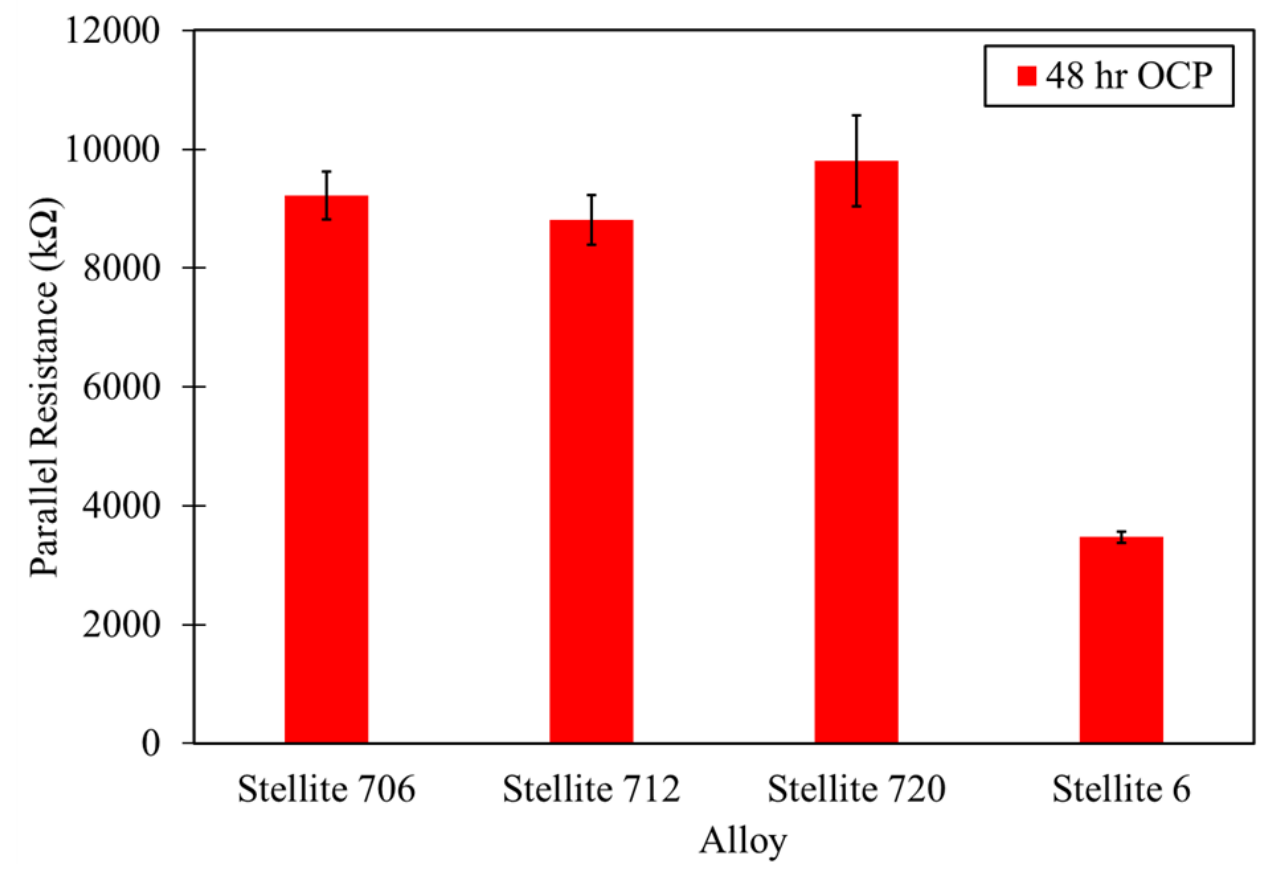

(a)

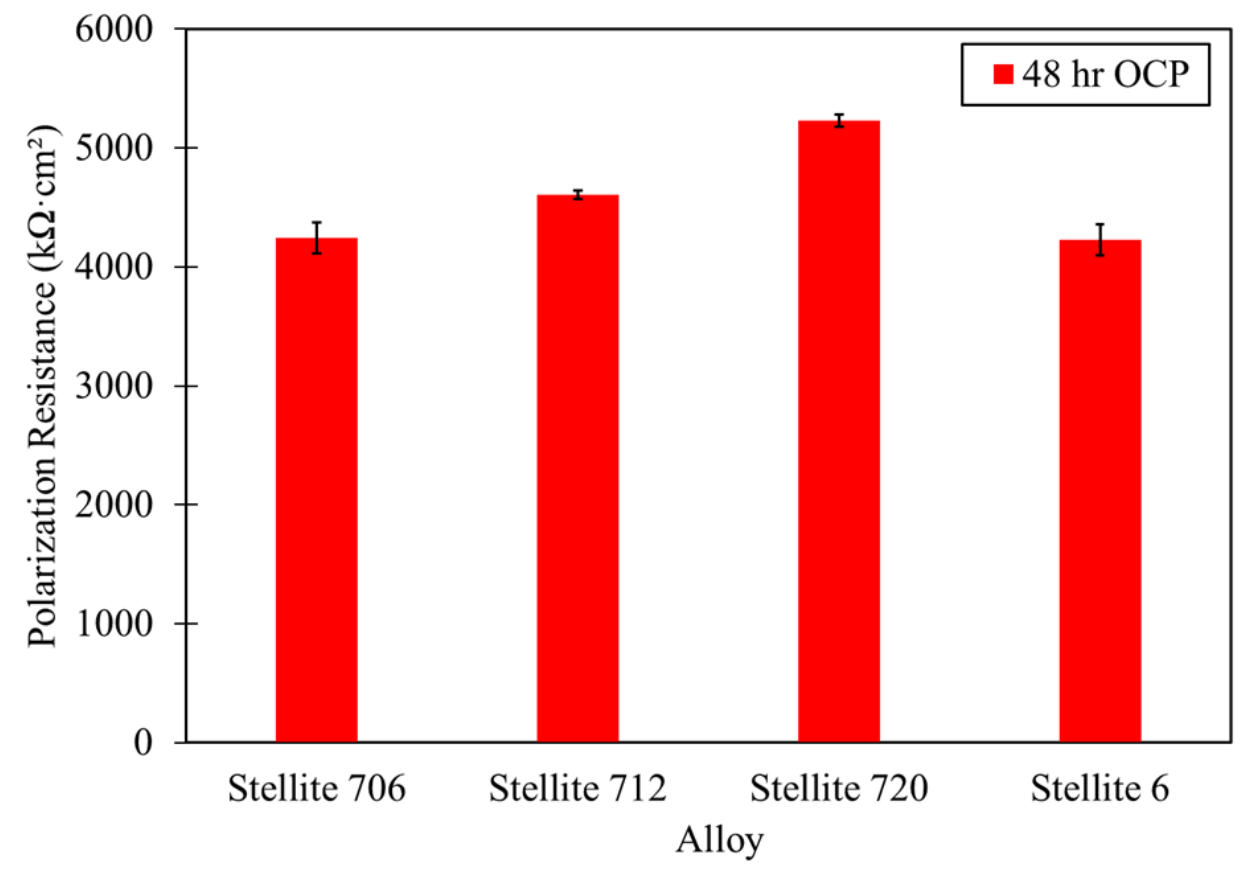

(b)

Figure 4-72: Overall comparison of all Stellite alloys for (a) EIS and (b) polarization after 48 hours OCP in morpholine 


\subsection{Discussion}

\subsubsection{Oxide film}

Stellite alloys are generally corrosion-resistant due to their high $\mathrm{Cr}$ content, which enables the formation of adherent oxide films on their surfaces. EIS tests showed that the oxide film for all 700 series Stellite alloys displays better protective properties with increasing OCP duration. This result was analogous in polarization tests, where the general corrosion properties also improved. Polarization tests also showed that the three Stellite alloys have the capability to form a spontaneous passivation region and transpassive region. All the Stellite alloys including Stellite 6 exhibited excellent corrosion resistance in morpholine solution with $\mathrm{pH}$ 9.5. In addition, the oxides are formed mainly in the $\mathrm{CoCr}$ solid solution region and the $(\mathrm{Co}, \mathrm{Mo})_{3} \mathrm{C}$ intermetallic regions. The Cr carbides can be oxidized, however not to a degree compared to the rest of the matrix. This has been confirmed from the EDS analyses of the corroded surfaces where large concentrations of O were found in the entire matrix except within the Cr carbide regions.

In summary, the Stellite alloys tested in morpholine has excellent general and localized corrosion resistance properties where EIS show strong oxide integrity and cyclic polarization show stable hysteresis at higher potential values. Also, from the failure tests, these alloys display excellent localized corrosion resistance in both morpholine and $\mathrm{NaCl}$, since under high potentials the alloy surfaces did not catastrophically fail. Overall the best performance amongst all the Stellite alloys in terms of oxide film stability is Stellite 720, which has the highest Mo content.

\subsubsection{Alloying elements}

Stellite 706, Stellite 712 and Stellite 720 are Co-based alloys with Cr, Mo and C as the main alloying elements. The electrochemical corrosion tests show that the overall corrosion resistance of these three alloys in morpholine solution with $\mathrm{pH} 9.5$ is close to one another with Stellite 720 
showing the best performance. Looking at the values from Table 4-1, Table 4-3 and their corresponding bar graph figures, Stellite 720 had better performance at $25^{\circ} \mathrm{C}$ while Stellite 706 had better performance at $50^{\circ} \mathrm{C}$. Considering the detrimental effect of the presence of carbides on corrosion, Stellite 706 should be better because it has the least amount of carbides. However, the only possible mechanism for better performance of Stellite 720 at $25^{\circ} \mathrm{C}$ for all OCP durations is the beneficial effect of Mo on corrosion.

From the comparative tests, Stellite 6 , containing $4.5 \mathrm{wt} . \% \mathrm{~W}$ and $1.5 \mathrm{wt} . \% \mathrm{Mo}$, behaved similarly to Stellite 706, containing 5 wt.\% Mo, qualitatively in morpholine solution with pH 9.5. They all exhibited excellent general and localized corrosion resistance including from the morpholine failure tests. However, the EIS results showed that Stellite 706 had increasingly better oxide stability compared to Stellite 6 with respect to the $R_{p}$ value. Stellite 6 reached its peak performance after 24 hours OCP and did not improve further. This suggests that the elements $\mathrm{W}$ and Mo function similarly in influencing the corrosion resistance of Stellite alloys in morpholine solution with $\mathrm{pH}$ 9.5, where Mo addition displays better results compared to $\mathrm{W}$.

\subsubsection{Elevated temperature}

Oxidation is a process in which elements are taken out of the substrate, thus consuming the material. Stellite alloys being a multi-phase system with $\mathrm{Cr}$-rich carbides and $(\mathrm{Co}, \mathrm{Mo})_{3} \mathrm{C}$ intermetallic regions have interfaces that are high energy sites. With increasing $\mathrm{C}$ and Mo, the interfaces increase, which means more grain boundaries are present. In electrochemical corrosion, the grain boundary regions behave as a battery to allow galvanic corrosion to proceed, hence fewer carbides in Stellite alloys should improve corrosion resistance at higher temperatures due to fewer interfaces. Polarization tests in morpholine solution with $\mathrm{pH} 9.5$ at $50^{\circ} \mathrm{C}$ showed Stellite 706 exhibited better corrosion resistance amongst the others. Generally, increasing temperature 
promotes atomic motion of the materials in the corrosion solution and thus expedites the chemical reactions in the electrochemical system. From each repeat experiments done, Stellite 706 consistently exhibited slightly better performance than the others. The detrimental effect of carbides can be offset by the addition of Mo, however, lack of interfaces at elevated temperatures ultimately show better performance.

\subsubsection{Worn surface}

The corrosion tests conducted on worn specimens were to simulate the practical service condition of the Stellite alloys, because valve trim components in application are subjected to synergetic loading of wear and corrosion. After wear, the alloy surfaces were damaged, with plastic deformation, residual stress, roughness, etc., which can alter the oxide behaviour on the alloy surfaces. However, due to too many random factors in the worn surfaces, it is difficult to relate the wear damage with the corrosion performance of the alloys, but in this study, the worn Stellite alloys did not show decreased corrosion resistance at $25^{\circ} \mathrm{C}$. The possible reason for the slight increase in performance can be due to wear generated heat at the contact surface, which may have promoted thicker oxide film formation in the alloy surface. At $50^{\circ} \mathrm{C}$, the corrosion performance of worn samples did not have significant differences compared to the intact surface.

\subsubsection{Pourbaix diagram}

Pourbaix diagram is a representation of a metals thermodynamic equilibrium phases in a water based electrolyte system. In electrochemistry, when redox reactions are happening, there is no practical way to know what exact reactions are taking place in the system. Thus, pourbaix diagrams can help identify the stable phases each individual metals can have during dissolution. Pourbaix diagrams show a metals stable state with increasing potential as a function of $\mathrm{pH}$. In this study, the DC input potentials were taken up to $1.2 \mathrm{~V}$ initially at $9.5 \mathrm{pH}$ and from the pourbaix diagrams 
given in Figure 4-73 to Figure 4-75 show the stable states of $\mathrm{Mo}, \mathrm{Cr}$ and $\mathrm{Co}$ are in up to that peak potential. At the spontaneous passive range the $\mathrm{Cr}$ is in $\mathrm{Cr}_{2} \mathrm{O}_{3}$ state and at the transpassive, it is at $\mathrm{CrO}_{4}{ }^{2-}$ state. Mo is in $\mathrm{MoO}_{4}{ }^{2-}$ state for most of the potential range. The state of Co will be at $\mathrm{Co}(\mathrm{OH})_{2}$ at the lower potentials and $\mathrm{Co}(\mathrm{OH})_{3}$ for the higher potentials. In the case of elevated temperatures and failure tests, the chemical state of each individual elements is unknown, as the limitation of pourbaix diagrams only apply to pure metals in standard temperature and pressure conditions (STP). Thermodynamic conditions for alloys differ when dealing with non-standard conditions and in the case of failure tests in $\mathrm{NaCl}$, the kinetics of the corrosion reaction cannot be determined using pourbaix diagrams. However, for room temperature conditions in morpholine, pourbaix diagrams help access the possible chemical states each element can be in theoretically.

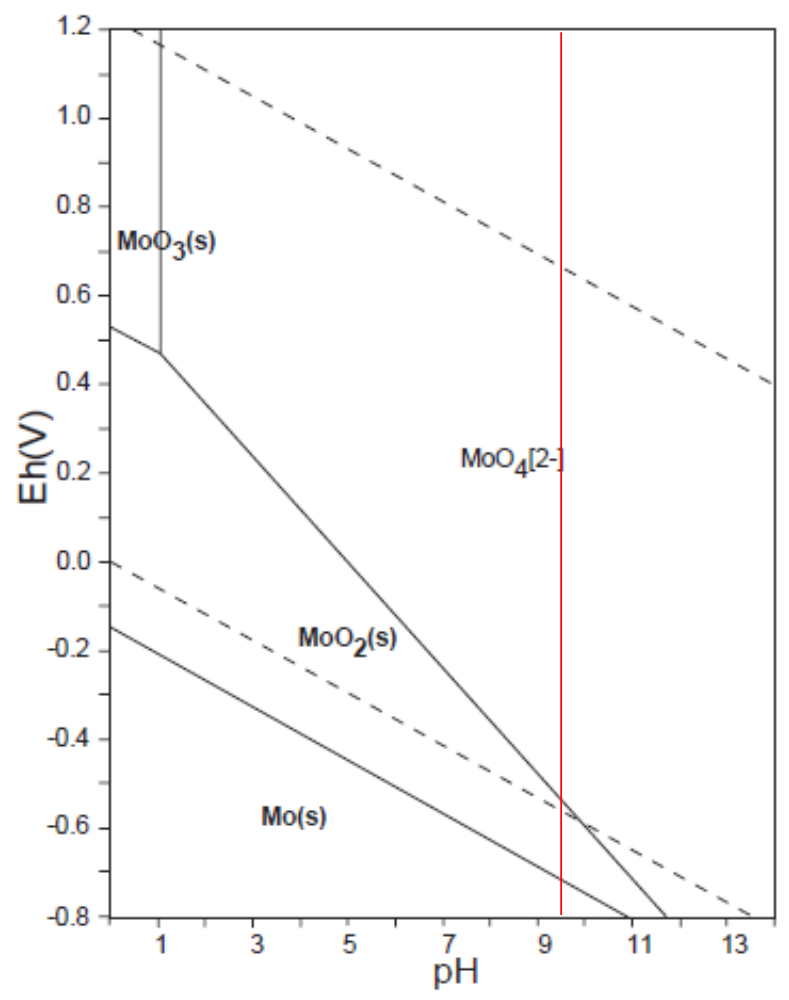

Figure 4-73: Pourbaix diagram of Molybdenum-water system [38] 


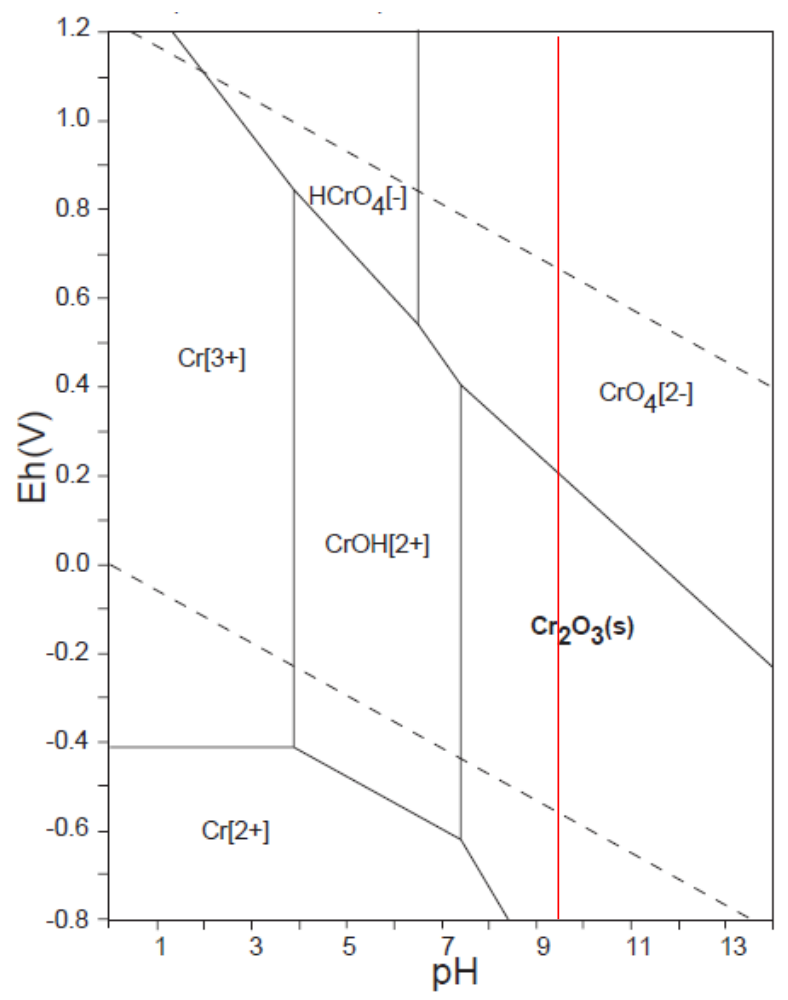

Figure 4-74: Pourbaix diagram for Chromium-water system [38]

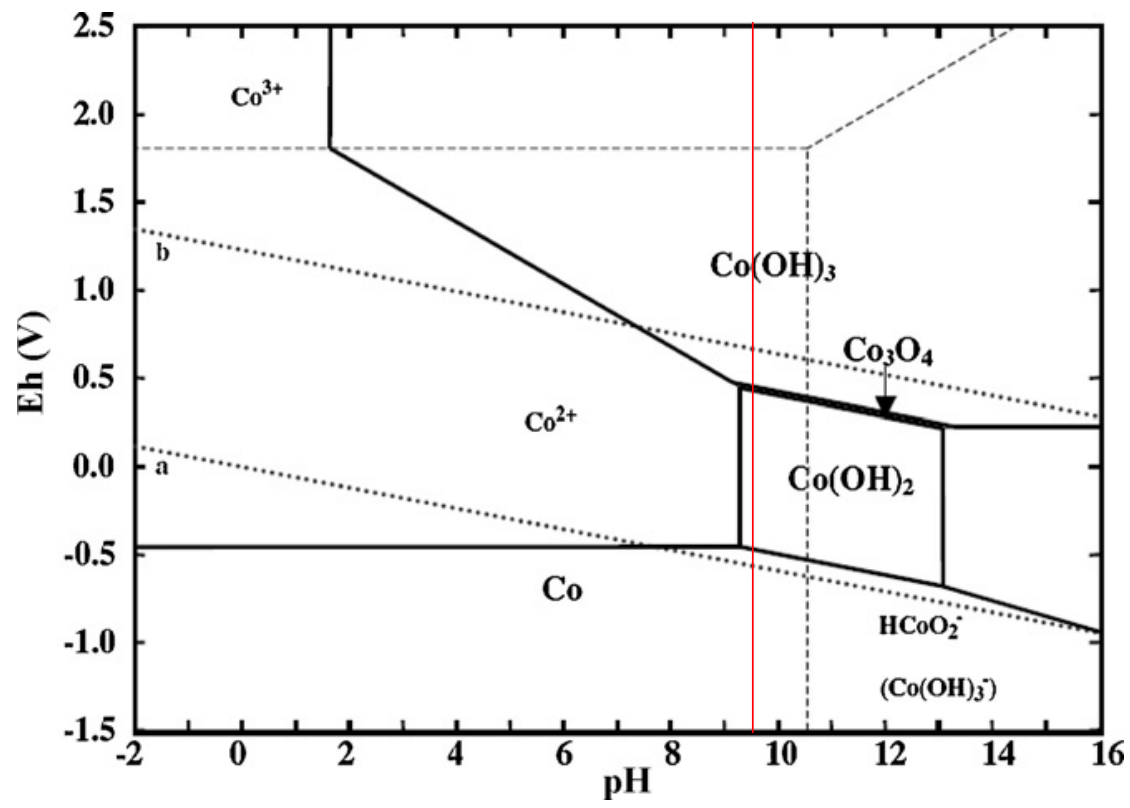

Figure 4-75: Pourbaix diagram for Cobalt-water system [48] 


\section{Chapter: Conclusions and Future Work}

\subsection{Summary}

To find suitable replacements of Stellite 6 alloy for corrosion resistance in amine environment, 700 series Stellite alloys — Stellite 706, Stellite 712 and Stellite 720 were studied in morpholine solution with $\mathrm{pH} 9.5$ using electrochemical tests such as EIS, potentiodynamic polarization and potentiostatic polarization at two different surface conditions and two different temperature conditions initially. The alloys were also subjected to different OCP times for a more accurate comparison near their peak performance. Failure tests in morpholine and $3.5 \mathrm{wt} . \% \mathrm{NaCl}$ were also done to test the alloy limits. The microstructure before and after corrosion was examined using SEM image analysis and the corroded surface was also examined for qualitative analysis using EDS. Stellite 6 was also tested under the same OCP and failure conditions for comparison. The experimental results were analyzed and discussed.

\subsection{Conclusions}

From the observations of these experiments and the analyses of the results as well as the discussion, the following conclusions can be derived:

1. Stellite 706 , Stellite 712 and Stellite 720 all exhibited excellent corrosion resistance in morpholine solution with $\mathrm{pH} 9.5$, by forming a protective $\mathrm{Cr}$-rich oxide film on their surfaces, which was retained well at both low and high peak potentials. Longer OCP time allowed the oxide film to further stabilize, thus enhancing both oxide film integrity and general corrosion resistance of the alloys.

2. Stellite 720 containing the highest $\mathrm{C}$ and Mo contents, performed better than the others at $25^{\circ} \mathrm{C}$ and Stellite 706 , containing the lowest $\mathrm{C}$ and lowest Mo contents, behaved slightly 
better at $50^{\circ} \mathrm{C}$. This can be the result of the compromise between beneficial Mo effect and detrimental carbide effect.

3. The worn alloys did not exhibit worse corrosion behaviour compared to the intact alloys in morpholine solution with $\mathrm{pH} 9.5$. The worn surfaces contain residual stresses and plastic deformations, which did not significantly affect the oxide film integrity and general corrosion performance under the $1.2 \mathrm{~V}$ peak applied polarization tests.

4. Due to the excellent performance of the oxide films, pitting corrosion did not occur on Stellite 706, Stellite 712 and Stellite 720 in morpholine solution with $\mathrm{pH} 9.5$ for all OCP durations as the hysteresis loop showed stable behaviour.

5. Stellite 706 , Stellite 712 and Stellite 720 are corroded in $3.5 \mathrm{wt} \% \mathrm{NaCl}$ to test their limitations. Stellite 720 exhibited better performance from the stable hysteresis behaviour.

6. 700 series Stellite alloys display better performance in both EIS and polarization tests compared to Stellite 6 in morpholine solution with $\mathrm{pH} 9.5$ for the highest OCP duration in $25^{\circ} \mathrm{C}$.

\subsection{Research Implications}

From previous literature studies in stainless steel, it was reported that Mo had an influence on corrosion performance via protecting the active sites by slowing down dissolution due to forming complex chlorides. Although solubility limit of Mo is not known, the information present so far is that 18 wt. $\%$ Mo makes a difference compared to the 8.5 wt.\%. Customers that purchase Stellite alloys for use in valve trim components can make purchasing decisions based on specific criteria's and constraints. Cost is an important consideration in choosing which Stellite alloy will be a better investment in the long run. A short and simple analysis can be done by looking at the weighted 
average cost for each major alloying element for Stellite alloys. The weighted average cost can be calculated using the following formula:

$$
W A C=\frac{\text { Summation } \text { cost of alloying elements }}{\text { Total composition }}
$$

The summation cost includes the total cost of $\mathrm{Co}, \mathrm{Cr}$ and Mo multiplied by their composition. Although $\mathrm{C}$ is also a major alloying element, its cost impact is minor compared to the other three. Using this equation, a historical graph can be constructed to show a relative total cost per metric ton of the major materials needed to synthesize the alloy assuming that the manufacturing process cost is constant. Three different sources [42-44] are looked at to obtain the cost of each element and Figure 5-1 shows their historical costs to present.

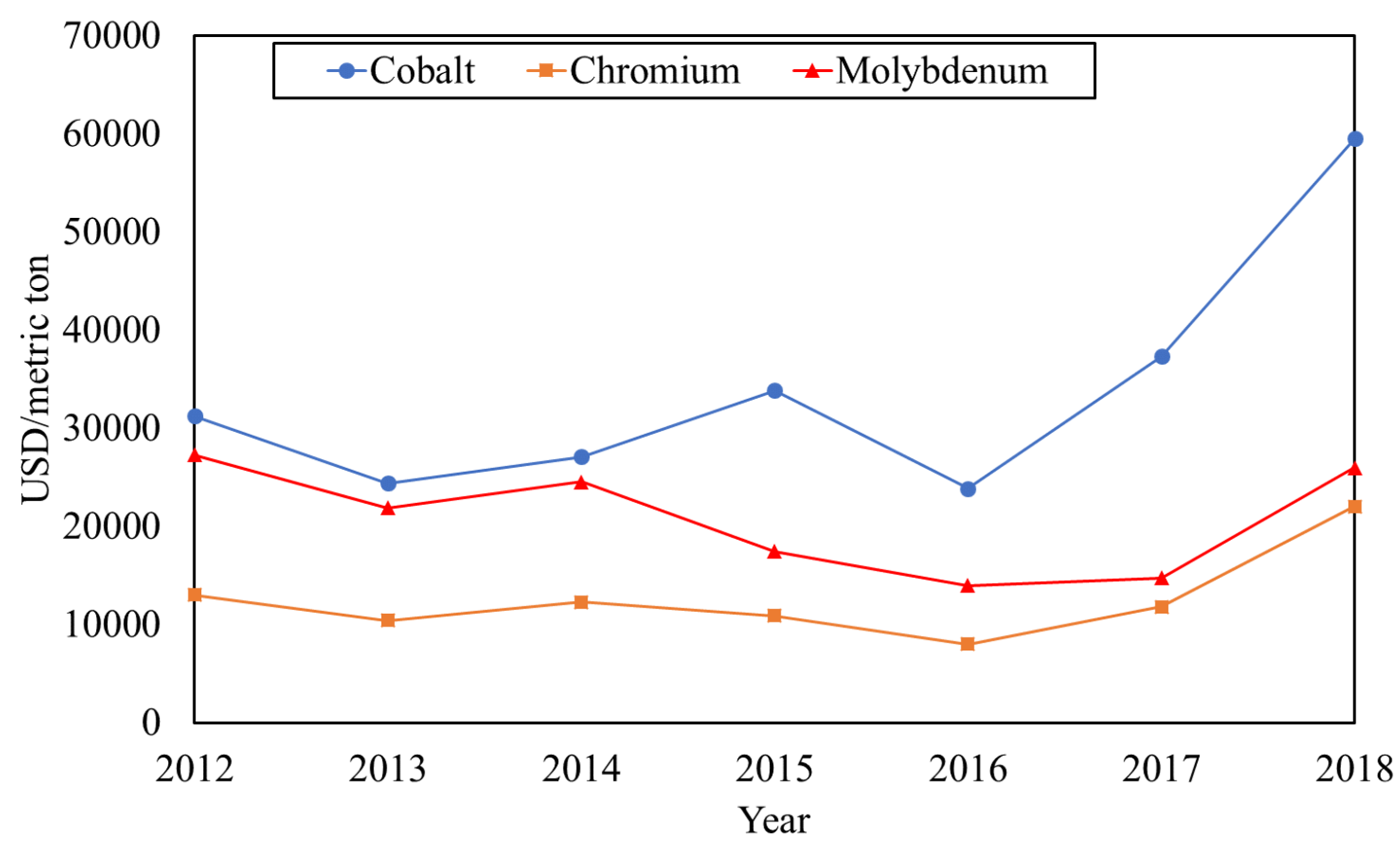

Figure 5-1: Historical costs of $\mathrm{Co}, \mathrm{Cr}$ and $\mathrm{Mo}$ in the past six years 
Looking at previous years up until recently, Co has always been the most expensive element followed by Mo. Using Eq (18) the weighted average cost for each Stellite alloy is shown in Figure 5-2 and given that Co is the most expensive element, it makes sense for Stellite 720 to be the cheapest among the three alloys under study since it has the lowest Co balance.

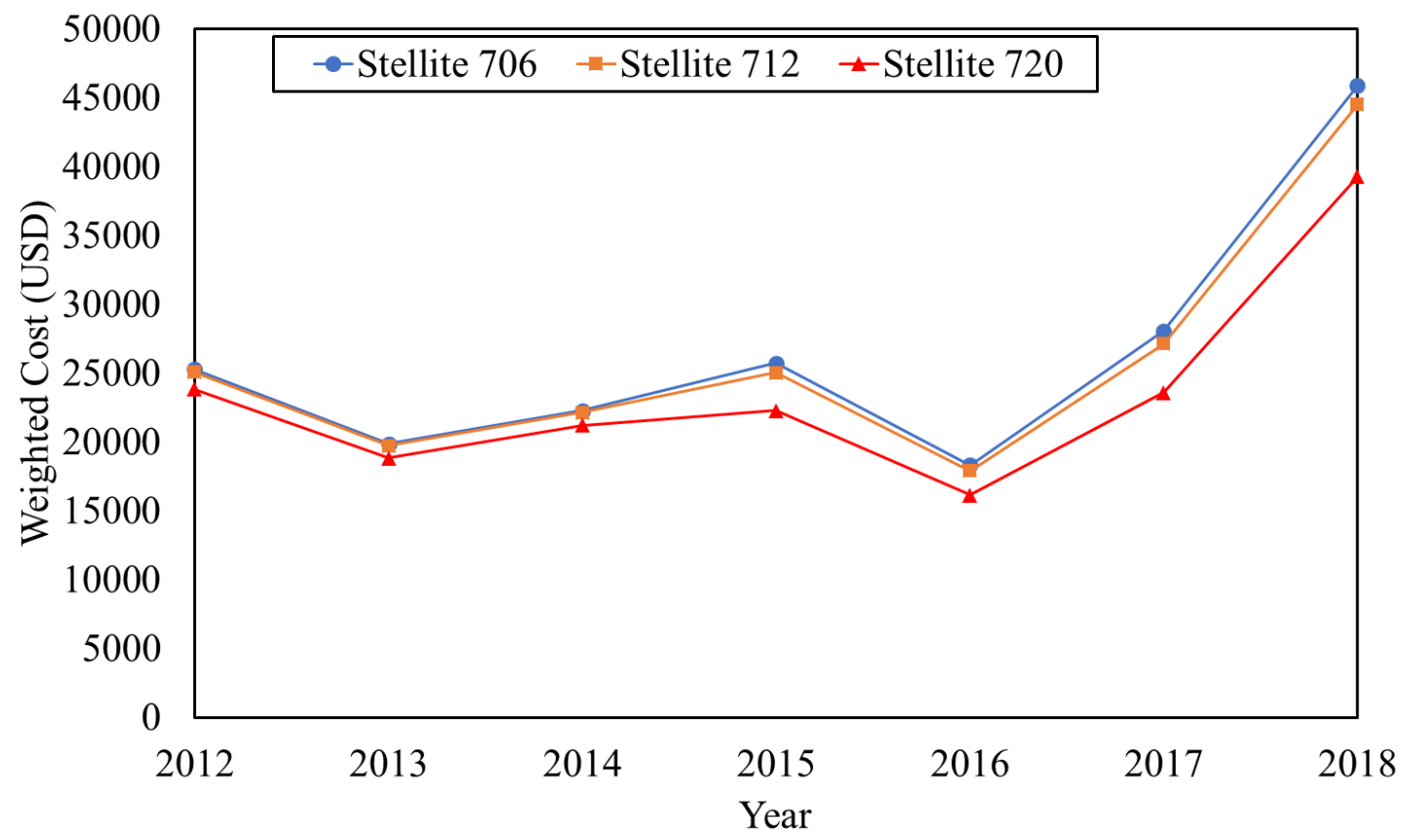

Figure 5-2: Historical costs of the Stellite alloys under study

Since results have already shown that Stellite 720 has the better performance in salt electrolyte, it can be the recommended alloy for replacing Stellite 6 for the hardfacing. Any customer looking to buy Stellite alloys from Kennametal Stellite Inc. can make their case for purchase depending on chemistry control and probability of excursion. Chemistry control and incursion probability depends on many problems that may arise in boiler feedwater treatments systems such as impurities, drop in $\mathrm{pH}$ and among others. If industries that use boiler feedwater treatment systems has confidence in their systems chemistry control of the feedwater where proper regulations are 
met in terms of chloride ion concentration, then any of the 700 series Stellite alloys including Stellite 6 can be used in application. However, if probability of excursion is relatively high, then Stellite 720 would be ultimately the best choice for maximum performance. However, this conclusion only applies to the conditions in which the alloys were tested in from this study. The effect of 700 series Stellite alloys along with Stellite 6 at multiple variations of conditions are unknown and possible future works are listed in the next section.

\subsection{Future Work}

\subsubsection{Process fluid flow rate, temperature and pressure}

To better simulate application, Stellite alloy should be tested with variable flow rates, pressures and temperatures as a function of time for a more comprehensive analysis. The valve trim components in application are exposed to such varying conditions, which eventually lead to erosion-corrosion failure. The goal here would be to analyze the Stellite alloys more efficiently with lesser limitations. This research had limitations where the corrosion tests on the Stellite alloys were performed under stagnant conditions for two temperature variables. With more variables for testing, the information obtained will be more comprehensive.

\subsubsection{Chemistry of the oxide film}

Information on how the oxide film chemistry changes for different conditions can be beneficial. It is known from previous researches that Mo ions such as $\mathrm{Mo}^{4+}$ and $\mathrm{Mo}^{6+}$ have a role in slowing dissolution and further protecting active sites. By using XPS technique, information of Mo and its interaction with $\mathrm{Co}, \mathrm{Cr}$ and $\mathrm{Cl}$ can be obtained for varying temperatures, pressures and flow rates. Different concentration ranges of $\mathrm{NaCl}$ electrolyte solution can be used in order to gain an understanding of the limitations for different compositions of Mo. 


\subsubsection{Comparison with different amines}

Other amines such as cyclohexylamine and diethylaminoethanal are used to maintain alkalinity of feedwater. A better comparison is needed to justify if Stellite alloys in different amine conditions have either similar or different performance.

\subsubsection{Stellite 6 and variable $W$ based compositions}

Worn surface behaviour of Stellite 6 can be studied and compared to the intact surface to see if it has any changes in performance. Also, Stellite alloys with variable W composition can be tested under variable conditions to properly compare them with Mo. Historically additions of W was studied for wear resistance purposes and it would be beneficial to accumulate data on whether W and Mo alone or different combinations of the two elements can help formulate an optimum alloy that offers both good corrosion and wear resistance in different conditions. 


\section{References}

1. J. R. Davis and ASM International, Eds., Nickel, cobalt, and their alloys. Materials Park, OH: ASM International, 2000.

2. R. Liu, J. Yao, Q. Zhang, M. X. Yao, and R. Collier, "Effects of molybdenum content on the wear/erosion and corrosion performance of low-carbon Stellite alloys," Materials \& Design, vol. 78, pp. 95-106, Aug. 2015.

3. Kennametal Stellite, "Stellite Wear Solution," Brochure, 2016.

4. T. Spence, "Cobalt-based Alloy 6 Materials and Boiler Feedwater Service," 16-Apr2008. [Online]. Available: http://www.valvemagazine.com/magazine/sections/materialsq-a/4271-cobalt-based-alloy-6-materials-and-boiler-feedwater-service.html.

5. Y. Li, "Electrochemical Study of Corrosion Behavior of Stellite 6 Alloy and 17-4PH Stainless Steel in Amine Solutions," Carleton University, Ottawa, 2017.

6. X. Z. Zhang, R. Liu, K. Y. Chen, M. X. Yao, and R. Collier, "Electrochemical Study of Corrosion Behavior of Wrought Stellite Alloys in Sodium Chloride and Green Death Solutions," Journal of Materials Engineering and Performance, vol. 24, no. 9, pp. 35793587, Sep. 2015.

7. M. X. Yao, J. B. C. Wu, and Y. Xie, "Wear, corrosion and cracking resistance of some W- or Mo-containing Stellite hardfacing alloys," Materials Science and Engineering: A, vol. 407, no. 1-2, pp. 234-244, Oct. 2005.

8. U. Malayoglu and A. Neville, "Mo and W as alloying elements in Co-based alloys - their effects on erosion-corrosion resistance," Wear, vol. 259, no. 1-6, pp. 219-229, Jul. 2005.

9. S. Y. Suslov et al., "Complex amine-based reagents," Thermal Engineering, vol. 64, no. 3, pp. 237-241, Mar. 2017.

10. X. Zhang, "Investigation of Corrosion Behavior of Wrought Stellite Alloys.," Carleton University, Ottawa, 2014.

11. R. Liu, J. H. Yao, Q. L. Zhang, M. X. Yao, and R. Collier, "Microstructures and Hardness/Wear Performance of High-Carbon Stellite Alloys Containing Molybdenum," Metallurgical and Materials Transactions A, vol. 46, no. 12, pp. 5504-5513, Dec. 2015.

12. W. D. Callister, Materials science and engineering: an introduction, 7 th ed. New York: John Wiley \& Sons, 2007.

13. D. Askeland, P. Fulay and W. Wright, The science and engineering of materials, 6 th ed. Stamford: Global Engineering, 2011.

14. "Boiler Chemicals 101," N.C. Division of Pollution Prevention and Environmental Assistance, Jun. 2004.

15. L. Pincus, Practical Boiler Water Treatment. United States: McGraw-Hill, 1962.

16. Samco, "All You Need to Know About Boiler Feed Water Treatment," Samco

17. U.S National Library of Medicine, "Morpholine," National Center for Biotechnology Information. [Online]. Available: https://pubchem.ncbi.nlm.nih.gov/compound/morpholine\#section=Top. [Accessed: 06Jun-2018].

18. F. Rosalbino and G. Scavino, "Corrosion behaviour assessment of cast and HIPed Stellite 6 alloy in a chloride-containing environment," Electrochimica Acta, vol. 111, pp. 656662, Nov. 2013. 
19. S.-S. Chang, H.-C. Wu, and C. Chen, "Impact Wear Resistance of Stellite 6 Hardfaced Valve Seats with Laser Cladding," Materials and Manufacturing Processes, vol. 23, no. 7, pp. 708-713, Sep. 2008.

20. P. S. Hu, R. Liu, J. Liu, and G. McRae, "Investigation of Wear and Corrosion of a HighCarbon Stellite Alloy for Hip Implants," Journal of Materials Engineering and Performance, vol. 23, no. 4, pp. 1223-1230, Apr. 2014.

21. ASTM. "ASTM G102 - 89(2015)e1." ASTM International - Standards Worldwide. ASTM International, 2015. Web. 12 Jan. 2017.

22. N. Perez, "Electrochemistry and corrosion science", Kluwer Academic Publishers, 2004.

23. ASTM. "ASTM G61 - 86(2014)." ASTM International - Standards Worldwide. ASTM International, 2014. Web. 11 Jan. 2017.

24. Gamry. "Review of the Electrochemical Basis of Corrosion." Getting Started with Electrochemical Corrosion Measurement. Gamry Instruments, n.d. Web. 14 Apr. 2017.

25. M. Ali, "Studies of the Use of Electrochemical Impedance Spectroscopy to Characterize and Assess the Performance of Lacquers Used to Protect Aluminum Sheet and Can Ends.," Carleton University, Ottawa, 2014.

26. Gamry. "Basics of Electrochemical Impedance Spectroscopy." Gamry Instruments. N.p., n.d. Web. 29 June 2017.

27. ASTM. "ASTM G5 - 14." ASTM International - Standards Worldwide. ASTM International, 2014. Web. 11 Jan. 2017.

28. ASTM. "ASTM G3 - 14." ASTM International - Standards Worldwide. ASTM International, 2014. Web. 21 Jan. 2017.

29. Enos, David G., and Louie L. Scribner. "Tech Note 33." The Potentiodynamic Polarization Scan. Solartron Analytical, Jan. 1997. Web. 16 May 2017.

30. MIT. "The Metallographic Examination of Archaeological Artifacts." Summer Institute in Materials Science and Materials Culture, MIT, 2003

31. Klarstrom, D., et al. "Metallography and Microstructures of Cobalt and Cobalt Alloys." Metallography and Microstructures of Cobalt and Cobalt Alloys - ASM International, ASM International, 2004, www.asminternational.org/search//journal_content/56/10192/ASMHBA0003771/BOOK-ARTICLE.

32. C. T. Sims, A contemporary view of cobalt-base alloys JOM, 21, 1969, 27-42.

33. L. F. Garfias-Mesias, J. M. Sykes, and C. D. S. Tuck, "The effect of phase compositions on the pitting corrosion of $25 \mathrm{Cr}$ duplex stainless steel in chloride solutions," Corrosion Science, vol. 38, no. 8, pp. 1319-1330, Aug. 1996.

34. S. J. Morrow, "MATERIALS SELECTION FOR SEAWATER PUMPS," p. 8, 2010.

35. I. Olefjord, "Surface Composition of Stainless Steels during Anodic Dissolution and Passivation Studied by ESCA," Journal of The Electrochemical Society, vol. 132, no. 12, p. 2854, 1985.

36. K. Sugimoto and Y. Sawada, Corrosion, 32, 347 (1976).

37. Hashimoto, K. Asami, and K. Teramoto, Corros. Sci.,19, 3 (1973).

38. N. Takeno, "Atlas of Eh-pH diagrams Intercomparison of thermodynamic databases," 2005

39. S. Masahiro, Electrochemical Society, and Corrosion Division, Passivity and Localized Corrosion: An International Symposium in Honor of Professor Norio Sato, vol. 99. The Electrochemical Society, 1999. 
40. J. M. Bastidas, C. L. Torres, E. Cano, and J. L. Polo, "Influence of molybdenum on passivation of polarised stainless steels in a chloride environment," Corrosion Science, p. 9, 2002.

41. M. N. Majeed, "Understanding the Erosion - Corrosion Behaviour of Generic Types of Stainless Steels in a CO2 - Saturated Oilfield Environment."

42. "Commodities Prices - Spot - Futures," Trading Economics, 2018. [Online]. Available: https://tradingeconomics.com/commodities.

43. "Daily Metal Prices," Metalary, 2018. [Online]. Available: https://www.metalary.com/.

44. "Commodity Closing Prices," InvestmentMine, 2018. [Online]. Available: http://www.infomine.com/investment/.

45. D. R. Lide, G. Baysinger, S. Chemistry, L. I. Berger, R. N. Goldberg, and H. V. Kehiaian, CRC Handbook of Chemistry and Physics, 2661 vols.

46. R. Burden and J. D. Faires, "Chapter 4: Numerical Differentiation and Integration," in Numerical Analysis, 9th ed., Brooks/Cole, 2011, p. 194.

47. H. H. Uhlig and R. W. Revie, "Boiler-Water Treatment for Corrosion Control," in Corrosion and Corrosion Control: An Introduction to Corrosion Science and Engineering, Third Edition., John Wiley \& Sons, 1985, pp. 288-290.

48. E. M. Garcia, J. S. Santos, E. C. Pereira, and M. B. J. G. Freitas, "Electrodeposition of cobalt from spent Li-ion battery cathodes by the electrochemistry quartz crystal microbalance technique," Journal of Power Sources, vol. 185, no. 1, pp. 549-553, Oct. 2008. 